\title{
Photoredox-Catalyzed Hydroxymethylation of Heteroaromatic Bases
}

\author{
Chelsea A. Huff, ${ }^{a}$ Ryan D. Cohen, ${ }^{b}$ Kevin D. Dykstra, ${ }^{a}$ Eric Streckfuss, ${ }^{a}$ \\ Daniel A. DiRocco, ${ }^{\mathrm{b}, *}$ and Shane W. Krska ${ }^{\mathrm{a}, *}$ \\ ${ }^{a}$ Department of Medicinal Chemistry, Merck Research Laboratories, 90 E. Scott Ave., \\ Rahway, NJ 07065 (USA) \\ ${ }^{b}$ Department of Process Chemistry, Merck Research Laboratories, 90 E. Scott Ave., \\ Rahway, NJ 07065 (USA)
}

\section{Table of Contents}

I. Materials and Methods

II. General Procedures

A. High-Throughput Optimization of Hydroxymethylation Reaction

B. Evaluation of Photoredox-Mediated and Minisci Hydroxymethylation

C. Evaluation of Lewis Acids and Oxidants for the Hydroxymethylation Reaction

III. References 


\section{Materials and Methods}

${ }^{1} \mathrm{H}$ NMR spectra were recorded using either a 500 or $600 \mathrm{MHz}$ spectrometer. Data are reported as follows: chemical shifts in parts per million $(\delta, \mathrm{ppm})$ referenced to residual solvent signals, DMSO- $\mathrm{d}_{5}(2.50 \mathrm{ppm})$, methanol- $\mathrm{d}_{3}(3.31 \mathrm{ppm})$, or $\mathrm{CHCl}_{3}(7.26 \mathrm{ppm})$, multiplicity abbreviated as 's' (singlet), 'd' (doublet), 'dd' (doublet of doublets), 'ddd' (doublet of doublet of doublets), 't' (triplet), 'td' (triplet of doublets'), 'tt' (triplet of triplets), 'q' (quartet), 'm' (multiplet), 'o' (overlapped), and 'br' (broad), and coupling constants measured in $\mathrm{Hz} .{ }^{13} \mathrm{C}$ NMR spectra were recorded using either a 125 or $150 \mathrm{MHz}$ spectrometer. Chemical shifts are reported in parts per million $(\delta, \mathrm{ppm})$ referenced to solvent signals, DMSO- $\mathrm{d}_{6}(39.52 \mathrm{ppm})$, methanol- $\mathrm{d}_{4}(49.17 \mathrm{ppm})$, or $\mathrm{CDCl}_{3}(77.16$ ppm). ${ }^{19} \mathrm{~F}$ NMR spectra were recorded using either 470 or $574 \mathrm{MHz}$ spectrometers. In most cases, 2D homo- and heteronuclear experiments (COSY, multiplicity-edited HSQC, $(H / C) H M B C,(H / N) H M B C$, and ROESY) were needed to confirm the site of functionalization. The $J$ optimizations for $\mathrm{HSQC},(\mathrm{H} / \mathrm{C}) \mathrm{HMBC}$, and $(\mathrm{H} / \mathrm{N}) \mathrm{HMBC}$ were 145,10 , and $8 \mathrm{~Hz}$, respectively. ROESY mixing time was set to $300 \mathrm{~ms}$. All NMR experiments were performed at ambient temperature. HRMS data were obtained on a Waters Q-TOF Ultima API mass spectrometer. Reactions were monitored using a UPLC-MS. All reactions were carried out under nitrogen atmosphere in a glovebox or using standard Schlenk techniques. 4-methoxybenzoic peroxyanhydride was synthesized according to the literature protocol. ${ }^{1}$

A 96 vial reactor was used to evaluate various reaction conditions. The aluminum vial rack is designed to hold $968 \times 30 \mathrm{~mm}$ vials (Analytical Sales and Services, Inc. P\# $84001)$ containing parylene coated stir dowels $(1.98 \times 4.80 \mathrm{~mm})$ (V\&P Scientific, P\# 711D-1) and stirred via a V\&P Scientific (710E-2HM-1) tumble stirrer equipped with a 96 blue (InGaN, $120 \mathrm{~mW}$ ) LED array manipulated using a 15V DC controller. Reagents were dosed to each of the 96 vials as solutions via an Eppendorf pipette in a nitrogen filled glovebox. After sealing the vials using the aluminum vial rack cover equipped with silicone and PFA mats, the reactor was placed on top of the LED array and stirred via tumble stirring. The LED controller was set to $13 \mathrm{~mA}$ for $16 \mathrm{~h}$. A blue $36 \mathrm{~W}$ Kessil LED lamp was used for "scale-up" reactions. 


\section{General Procedures}

\section{A. High-Throughput Optimization of Hydroxymethylation Reaction}

Photocatalyst $(0.05 \mu \mathrm{mol}, 10 \mu \mathrm{L}$ of a $0.005 \mathrm{M}$ solution in ACN $), 4,4$ '-ditertbutylbiphenyl (internal standard, $1 \mu \mathrm{mol}, 40 \mu \mathrm{L}$ of a $0.025 \mathrm{M}$ solution in $\mathrm{ACN}$ ), and $\mathrm{BPO}$ or $\mathrm{NH}_{4} \mathrm{~S}_{2} \mathrm{O}_{8}$ $(10 \mu \mathrm{mol}, 20 \mu \mathrm{L}$ of an $0.50 \mathrm{M}$ solution) were added to the reaction vials in a 96 well plate as noted in Table S1. The solvent was removed and in an $\mathrm{N}_{2}$-filled glove box, lepidine $(5 \mu \mathrm{mol}, 15 \mu \mathrm{L}$ of a $0.33 \mathrm{M}$ solution in $\mathrm{MeOH}, 1$ eq), TFA $(3.8 \mu \mathrm{L}, 50 \mu \mathrm{mol}, 10$ eq), TBEC or TBPA $(10 \mu \mathrm{mol}, 12.5 \mu \mathrm{L}$ of a $0.8 \mathrm{M}$ solution in $\mathrm{MeOH}$ ), and $\mathrm{MeOH}$, acetone, DCM, or acetonitrile were added to achieve a total volume of $50 \mu \mathrm{L}$ or $0.1 \mathrm{M}$ (in substrate) concentration, as described below in Table S1. The plate was sealed and removed from the glovebox, where it was illuminated with blue light (13 mA for array of 96 lights) for 16 hours at room temperature. The reactions were then diluted with $75 \mu \mathrm{l}$ of $\mathrm{MeOH}$, and a $15 \mu \mathrm{l}$ aliquot was diluted into $700 \mu \mathrm{l}$ of MeOH for UPLC-MS analysis. \% yield determined using a relative UPLC response factor between $\mathbf{1 a}$ and the internal standard (a 5:1 molar ratio of 1a:IS gives a 3:1 ratio in UV peak area (1a:IS) measured at $215 \mathrm{~nm}$ ). Photographs of the reaction manifold are available in the supporting information of our previous publication. ${ }^{2}$ See Figure $\mathrm{S} 1$ for a pictoral representation of the results.

Note: During the course of the reaction the temperature of the vial rack elevates slightly from room temperature due to the absorption of light from the LED array and generally stabilizes at $<28{ }^{\circ} \mathrm{C}$.

Table S1. High-Throughput Analysis of Hydroxymethylation of Lepidine

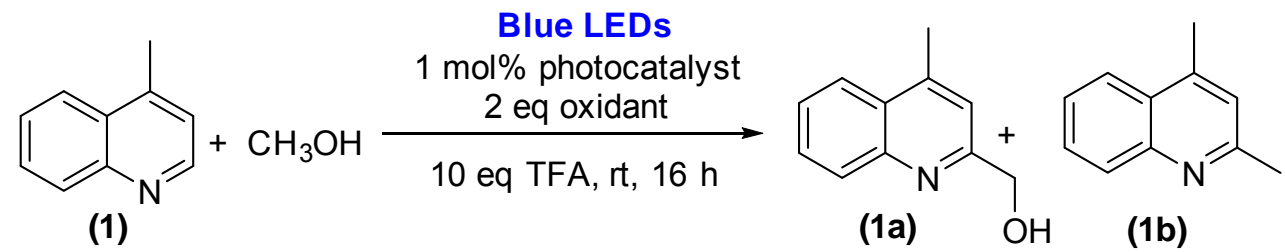

\begin{tabular}{|c|c|c|c|c|c|}
\hline Entry & Photocatalyst & Oxidant & Solvent & $\begin{array}{c}\text { \%Yield } \\
\text { 1a }\end{array}$ & $\begin{array}{c}\text { \%Yield } \\
\text { 1b }\end{array}$ \\
\hline A1 & Mes-Acr & TBPA & $\mathrm{MeOH}$ & 56 & 0 \\
\hline A2 & {$\left[\mathrm{Ru}(\mathrm{bpz})_{3}\right]\left(\mathrm{PF}_{6}\right)_{2}$} & TBPA & $\mathrm{MeOH}$ & 35 & 4 \\
\hline A3 & {$\left[\mathrm{Ru}(\mathrm{bpy})_{3}(\mathrm{PF} 6)_{2}\right.$} & TBPA & $\mathrm{MeOH}$ & 9 & 0 \\
\hline A4 & {$\left[\mathrm{Ru}(\mathrm{dip})_{2}\right] \mathrm{Cl}_{2}$} & TBPA & $\mathrm{MeOH}$ & 37 & 3 \\
\hline A5 & {$\left[\mathrm{Ir}\left(\mathrm{dF}-\mathrm{CF}_{3}-\mathrm{ppy}\right)_{2}(\mathrm{dtbpy})\right] \mathrm{PF}_{6}$} & TBPA & $\mathrm{MeOH}$ & 71 & 15 \\
\hline A6 & $\left.\operatorname{Ir}(\mathrm{ppy})_{2}(\mathrm{dtbpy})\right] \mathrm{PF}_{6}$ & TBPA & $\mathrm{MeOH}$ & 61 & 8 \\
\hline A7 & Mes-Acr & TBPA & $\mathrm{MeOH} / \mathrm{ACN}$ & 40 & 10 \\
\hline A8 & {$\left[\mathrm{Ru}(\mathrm{bpz})_{3}\right]\left(\mathrm{PF}_{6}\right)_{2}$} & TBPA & $\mathrm{MeOH} / \mathrm{ACN}$ & 12 & 4 \\
\hline A9 & {$\left[\mathrm{Ru}(\mathrm{bpy})_{3}(\mathrm{PF} 6)_{2}\right.$} & TBPA & $\mathrm{MeOH} / \mathrm{ACN}$ & 2 & 0 \\
\hline A10 & {$\left[\mathrm{Ru}(\mathrm{dip})_{2}\right] \mathrm{Cl}_{2}$} & TBPA & $\mathrm{MeOH} / \mathrm{ACN}$ & 22 & 3 \\
\hline A11 & {$\left[\mathrm{Ir}\left(\mathrm{dF}-\mathrm{CF}_{3}-\mathrm{ppy}\right)_{2}(\mathrm{dtbpy})\right] \mathrm{PF}_{6}$} & TBPA & $\mathrm{MeOH} / \mathrm{ACN}$ & 63 & 15 \\
\hline A12 & $\left.\operatorname{Ir}(p p y)_{2}(\mathrm{dtbpy})\right] \mathrm{PF}_{6}$ & TBPA & $\mathrm{MeOH} / \mathrm{ACN}$ & 46 & 10 \\
\hline
\end{tabular}




\begin{tabular}{|c|c|c|c|c|c|}
\hline B1 & Mes-Acr & TBEC & $\mathrm{MeOH}$ & 63 & 13 \\
\hline B2 & {$\left[\mathrm{Ru}(\mathrm{bpz})_{3}\right]\left(\mathrm{PF}_{6}\right)_{2}$} & TBEC & $\mathrm{MeOH}$ & 16 & 4 \\
\hline B3 & {$\left[\mathrm{Ru}(\mathrm{bpy})_{3}(\mathrm{PF} 6)_{2}\right.$} & TBEC & $\mathrm{MeOH}$ & 5 & 0 \\
\hline B4 & {$\left[\mathrm{Ru}(\mathrm{dip})_{2}\right] \mathrm{Cl}_{2}$} & TBEC & $\mathrm{MeOH}$ & 84 & 3 \\
\hline B5 & {$\left[\operatorname{lr}\left(\mathrm{dF}-\mathrm{CF}_{3}-\mathrm{ppy}\right)_{2}(\mathrm{dtbpy})\right] \mathrm{PF}_{6}$} & TBEC & $\mathrm{MeOH}$ & 63 & 15 \\
\hline B6 & $\left.\operatorname{Ir}(p p y)_{2}(d t b p y)\right] P F_{6}$ & TBEC & $\mathrm{MeOH}$ & 49 & 5 \\
\hline B7 & Mes-Acr & TBEC & $\mathrm{MeOH} / \mathrm{ACN}$ & 30 & 11 \\
\hline B8 & {$\left[\mathrm{Ru}(\mathrm{bpz})_{3}\right]\left(\mathrm{PF}_{6}\right)_{2}$} & TBEC & $\mathrm{MeOH} / \mathrm{ACN}$ & 10 & 5 \\
\hline B9 & {$\left[\mathrm{Ru}(\mathrm{bpy})_{3}(\mathrm{PF} 6)_{2}\right.$} & TBEC & $\mathrm{MeOH} / \mathrm{ACN}$ & 13 & 5 \\
\hline B10 & {$\left[\mathrm{Ru}(\mathrm{dip})_{2}\right] \mathrm{Cl}_{2}$} & TBEC & $\mathrm{MeOH} / \mathrm{ACN}$ & 57 & 2 \\
\hline B11 & {$\left[\mathrm{Ir}\left(\mathrm{dF}-\mathrm{CF}_{3}-\mathrm{ppy}\right)_{2}(\mathrm{dtbpy})\right] \mathrm{PF}_{6}$} & TBEC & $\mathrm{MeOH} / \mathrm{ACN}$ & 59 & 9 \\
\hline B12 & $\left.\operatorname{Ir}(p p y)_{2}(d t b p y)\right] P F_{6}$ & TBEC & $\mathrm{MeOH} / \mathrm{ACN}$ & 46 & 7 \\
\hline C1 & Mes-Acr & TBEC & $\mathrm{MeOH}$ & 45 & 0 \\
\hline $\mathrm{C} 2$ & {$\left[\mathrm{Ru}(\mathrm{bpz})_{3}\right]\left(\mathrm{PF}_{6}\right)_{2}$} & $\mathrm{NH}_{4} \mathrm{~S}_{2} \mathrm{O}_{8}$ & $\mathrm{MeOH}$ & 15 & 0 \\
\hline C3 & {$\left[\mathrm{Ru}(\mathrm{bpy})_{3}(\mathrm{PF} 6)_{2}\right.$} & $\mathrm{NH}_{4} \mathrm{~S}_{2} \mathrm{O}_{8}$ & $\mathrm{MeOH}$ & 61 & 0 \\
\hline $\mathrm{C} 4$ & {$\left[\mathrm{Ru}(\mathrm{dip})_{2}\right] \mathrm{Cl}_{2}$} & $\mathrm{NH}_{4} \mathrm{~S}_{2} \mathrm{O}_{8}$ & $\mathrm{MeOH}$ & 30 & 0 \\
\hline C5 & {$\left[\mathrm{Ir}\left(\mathrm{dF}-\mathrm{CF}_{3}-\mathrm{ppy}\right)_{2}(\mathrm{dtbpy})\right] \mathrm{PF} \mathrm{F}_{6}$} & $\mathrm{NH}_{4} \mathrm{~S}_{2} \mathrm{O}_{8}$ & $\mathrm{MeOH}$ & 95 & 4 \\
\hline C6 & $\left.\operatorname{Ir}(p p y)_{2}(\mathrm{dtbpy})\right] \mathrm{PF}_{6}$ & $\mathrm{NH}_{4} \mathrm{~S}_{2} \mathrm{O}_{8}$ & $\mathrm{MeOH}$ & 30 & 0 \\
\hline C7 & Mes-Acr & $\mathrm{NH}_{4} \mathrm{~S}_{2} \mathrm{O}_{8}$ & $\mathrm{MeOH} / \mathrm{ACN}$ & 30 & 0 \\
\hline $\mathrm{C} 8$ & {$\left[\mathrm{Ru}(\mathrm{bpz})_{3}\right]\left(\mathrm{PF}_{6}\right)_{2}$} & $\mathrm{NH}_{4} \mathrm{~S}_{2} \mathrm{O}_{8}$ & $\mathrm{MeOH} / \mathrm{ACN}$ & 6 & 0 \\
\hline C9 & {$\left[\mathrm{Ru}(\mathrm{bpy})_{3}(\mathrm{PF} 6)_{2}\right.$} & $\mathrm{NH}_{4} \mathrm{~S}_{2} \mathrm{O}_{8}$ & $\mathrm{MeOH} / \mathrm{ACN}$ & 14 & 0 \\
\hline C10 & {$\left[\mathrm{Ru}(\operatorname{dip})_{2}\right] \mathrm{Cl}_{2}$} & $\mathrm{NH}_{4} \mathrm{~S}_{2} \mathrm{O}_{8}$ & $\mathrm{MeOH} / \mathrm{ACN}$ & 7 & 0 \\
\hline C11 & {$\left[\operatorname{lr}\left(\mathrm{dF}-\mathrm{CF}_{3}-\mathrm{ppy}\right)_{2}(\mathrm{dtbpy})\right] \mathrm{PF}_{6}$} & $\mathrm{NH}_{4} \mathrm{~S}_{2} \mathrm{O}_{8}$ & $\mathrm{MeOH} / \mathrm{ACN}$ & 73 & 2 \\
\hline C12 & $\left.\operatorname{Ir}(p p y)_{2}(\mathrm{dtbpy})\right] \mathrm{PF}_{6}$ & $\mathrm{NH}_{4} \mathrm{~S}_{2} \mathrm{O}_{8}$ & $\mathrm{MeOH} / \mathrm{ACN}$ & 27 & 0 \\
\hline D1 & Mes-Acr & BPO & $\mathrm{MeOH}$ & 96 & 0 \\
\hline $\mathrm{D} 2$ & {$\left[\mathrm{Ru}(\mathrm{bpz})_{3}\right]\left(\mathrm{PF}_{6}\right)_{2}$} & $\mathrm{BPO}$ & $\mathrm{MeOH}$ & 9 & 0 \\
\hline D3 & {$\left[\mathrm{Ru}(\mathrm{bpy})_{3}(\mathrm{PF} 6)_{2}\right.$} & BPO & $\mathrm{MeOH}$ & 23 & 0 \\
\hline D4 & {$\left[\mathrm{Ru}(\mathrm{dip})_{2}\right] \mathrm{Cl}_{2}$} & BPO & $\mathrm{MeOH}$ & 13 & 0 \\
\hline D5 & {$\left[\operatorname{lr}\left(\mathrm{dF}-\mathrm{CF}_{3}-\mathrm{ppy}\right)_{2}(\mathrm{dtbpy})\right] \mathrm{PF}_{6}$} & BPO & $\mathrm{MeOH}$ & 97 & 1 \\
\hline D6 & $\left.\operatorname{Ir}(p p y)_{2}(d t b p y)\right] P F_{6}$ & BPO & $\mathrm{MeOH}$ & 67 & 0 \\
\hline D7 & Mes-Acr & $\mathrm{BPO}$ & $\mathrm{MeOH} / \mathrm{ACN}$ & 89 & 0 \\
\hline D8 & {$\left[\mathrm{Ru}(\mathrm{bpz})_{3}\right]\left(\mathrm{PF}_{6}\right)_{2}$} & BPO & $\mathrm{MeOH} / \mathrm{ACN}$ & 11 & 0 \\
\hline D9 & {$\left[\mathrm{Ru}(\mathrm{bpy})_{3}(\mathrm{PF} 6)_{2}\right.$} & BPO & $\mathrm{MeOH} / \mathrm{ACN}$ & 11 & 0 \\
\hline D10 & {$\left[\mathrm{Ru}(\mathrm{dip})_{2}\right] \mathrm{Cl}_{2}$} & BPO & $\mathrm{MeOH} / \mathrm{ACN}$ & 4 & 0 \\
\hline D11 & {$\left[\mathrm{Ir}\left(\mathrm{dF}-\mathrm{CF}_{3}-\mathrm{ppy}\right)_{2}(\mathrm{dtbpy})\right] \mathrm{PF}_{6}$} & BPO & $\mathrm{MeOH} / \mathrm{ACN}$ & 95 & 0 \\
\hline D12 & $\left.\operatorname{Ir}(p p y)_{2}(d t b p y)\right] P F_{6}$ & BPO & $\mathrm{MeOH} / \mathrm{ACN}$ & 74 & 0 \\
\hline E1 & Mes-Acr & TBPA & $\mathrm{MeOH} /$ Acetone & 44 & 6 \\
\hline $\mathrm{E} 2$ & {$\left[\mathrm{Ru}(\mathrm{bpz})_{3}\right]\left(\mathrm{PF}_{6}\right)_{2}$} & TBPA & $\mathrm{MeOH} /$ Acetone & 18 & 3 \\
\hline E3 & {$\left[\mathrm{Ru}(\mathrm{bpy})_{3}(\mathrm{PF} 6)_{2}\right.$} & TBPA & MeOH/Acetone & 8 & 0 \\
\hline E4 & {$\left[\mathrm{Ru}(\mathrm{dip})_{2}\right] \mathrm{Cl}_{2}$} & TBPA & MeOH/Acetone & 24 & 2 \\
\hline E5 & {$\left[\mathrm{Ir}\left(\mathrm{dF}-\mathrm{CF}_{3}-\mathrm{ppy}\right)_{2}(\mathrm{dtbpy})\right] \mathrm{PF}_{6}$} & TBPA & $\mathrm{MeOH} /$ Acetone & 49 & 6 \\
\hline E6 & $\left.\operatorname{Ir}(p p y)_{2}(d t b p y)\right] P F_{6}$ & TBPA & $\mathrm{MeOH} /$ Acetone & 51 & 8 \\
\hline E7 & Mes-Acr & TBPA & $\mathrm{MeOH} / \mathrm{DCM}$ & 32 & 6 \\
\hline E8 & {$\left[\mathrm{Ru}(\mathrm{bpz})_{3}\right]\left(\mathrm{PF}_{6}\right)_{2}$} & TBPA & $\mathrm{MeOH} / \mathrm{DCM}$ & 15 & 2 \\
\hline E9 & {$\left[\mathrm{Ru}(\mathrm{bpy})_{3}(\mathrm{PF} 6)_{2}\right.$} & TBPA & $\mathrm{MeOH} / \mathrm{DCM}$ & 8 & 0 \\
\hline
\end{tabular}




\begin{tabular}{|c|c|c|c|c|c|}
\hline E10 & {$\left[\mathrm{Ru}(\operatorname{dip})_{2}\right] \mathrm{Cl}_{2}$} & TBPA & $\mathrm{MeOH} / \mathrm{DCM}$ & 15 & 0 \\
\hline E11 & {$\left[\mathrm{Ir}\left(\mathrm{dF}-\mathrm{CF}_{3}-\mathrm{ppy}\right)_{2}(\mathrm{dtbpy})\right] \mathrm{PF} \mathrm{F}_{6}$} & TBPA & $\mathrm{MeOH} / \mathrm{DCM}$ & 41 & 6 \\
\hline E12 & $\left.\operatorname{Ir}(\mathrm{ppy})_{2}(\mathrm{dtbpy})\right] \mathrm{PF}_{6}$ & TBPA & $\mathrm{MeOH} / \mathrm{DCM}$ & 38 & 6 \\
\hline F1 & Mes-Acr & TBEC & $\mathrm{MeOH} /$ Acetone & 46 & 10 \\
\hline $\mathrm{F} 2$ & {$\left[\mathrm{Ru}(\mathrm{bpz})_{3}\right]\left(\mathrm{PF}_{6}\right)_{2}$} & TBEC & $\mathrm{MeOH} /$ Acetone & 12 & 3 \\
\hline F3 & {$\left[\mathrm{Ru}(\mathrm{bpy})_{3}(\mathrm{PF} 6)_{2}\right.$} & TBEC & $\mathrm{MeOH} /$ Acetone & 7 & 0 \\
\hline F4 & {$\left[\mathrm{Ru}(\mathrm{dip})_{2}\right] \mathrm{Cl}_{2}$} & TBEC & $\mathrm{MeOH} /$ Acetone & 69 & 2 \\
\hline F5 & {$\left[\operatorname{Ir}\left(\mathrm{dF}-\mathrm{CF}_{3}-\mathrm{ppy}\right)_{2}(\mathrm{dtbpy})\right] \mathrm{PF}_{6}$} & TBEC & $\mathrm{MeOH} /$ Acetone & 57 & 5 \\
\hline F6 & $\left.\operatorname{Ir}(p p y)_{2}(\mathrm{dtbpy})\right] \mathrm{PF}_{6}$ & TBEC & $\mathrm{MeOH} /$ Acetone & 53 & 5 \\
\hline F7 & Mes-Acr & TBEC & $\mathrm{MeOH} / \mathrm{DCM}$ & 43 & 8 \\
\hline F8 & {$\left[\mathrm{Ru}(\mathrm{bpz})_{3}\right]\left(\mathrm{PF}_{6}\right)_{2}$} & TBEC & $\mathrm{MeOH} / \mathrm{DCM}$ & 8 & 2 \\
\hline F9 & {$\left[\mathrm{Ru}(\mathrm{bpy})_{3}(\mathrm{PF} 6)_{2}\right.$} & TBEC & $\mathrm{MeOH} / \mathrm{DCM}$ & 6 & 0 \\
\hline F10 & {$\left[\mathrm{Ru}(\mathrm{dip})_{2}\right] \mathrm{Cl}_{2}$} & TBEC & $\mathrm{MeOH} / \mathrm{DCM}$ & 34 & 1 \\
\hline F11 & {$\left[\operatorname{lr}\left(\mathrm{dF}-\mathrm{CF}_{3}-\mathrm{ppy}\right)_{2}(\mathrm{dtbpy})\right] \mathrm{PF}_{6}$} & TBEC & $\mathrm{MeOH} / \mathrm{DCM}$ & 55 & 5 \\
\hline F12 & $\operatorname{Ir}(p p y)_{2}(\mathrm{dtbpy}) \mathrm{PF}_{6}$ & TBEC & $\mathrm{MeOH} / \mathrm{DCM}$ & 51 & 6 \\
\hline G1 & Mes-Acr & TBEC & $\mathrm{MeOH} /$ Acetone & 36 & 0 \\
\hline $\mathrm{G} 2$ & {$\left[\mathrm{Ru}(\mathrm{bpz})_{3}\right]\left(\mathrm{PF}_{6}\right)_{2}$} & $\mathrm{NH}_{4} \mathrm{~S}_{2} \mathrm{O}_{8}$ & $\mathrm{MeOH} /$ Acetone & 3 & 0 \\
\hline G3 & {$\left[\mathrm{Ru}(\mathrm{bpy})_{3}(\mathrm{PF} 6)_{2}\right.$} & $\mathrm{NH}_{4} \mathrm{~S}_{2} \mathrm{O}_{8}$ & $\mathrm{MeOH} /$ Acetone & 13 & 0 \\
\hline G4 & {$\left[\mathrm{Ru}(\mathrm{dip})_{2}\right] \mathrm{Cl}_{2}$} & $\mathrm{NH}_{4} \mathrm{~S}_{2} \mathrm{O}_{8}$ & $\mathrm{MeOH} /$ Acetone & 14 & 0 \\
\hline G5 & {$\left[\operatorname{lr}\left(\mathrm{dF}-\mathrm{CF}_{3}-\mathrm{ppy}\right)_{2}(\mathrm{dtbpy})\right] \mathrm{PF}_{6}$} & $\mathrm{NH}_{4} \mathrm{~S}_{2} \mathrm{O}_{8}$ & MeOH/Acetone & 67 & 8 \\
\hline G6 & $\left.\operatorname{Ir}(p p y)_{2}(\mathrm{dtbpy})\right] \mathrm{PF}_{6}$ & $\mathrm{NH}_{4} \mathrm{~S}_{2} \mathrm{O}_{8}$ & $\mathrm{MeOH} /$ Acetone & 27 & 0 \\
\hline G7 & Mes-Acr & $\mathrm{NH}_{4} \mathrm{~S}_{2} \mathrm{O}_{8}$ & $\mathrm{MeOH} / \mathrm{DCM}$ & 34 & 0 \\
\hline G8 & {$\left[\mathrm{Ru}(\mathrm{bpz})_{3}\right]\left(\mathrm{PF}_{6}\right)_{2}$} & $\mathrm{NH}_{4} \mathrm{~S}_{2} \mathrm{O}_{8}$ & $\mathrm{MeOH} / \mathrm{DCM}$ & 3 & 0 \\
\hline G9 & {$\left[\mathrm{Ru}(\mathrm{bpy})_{3}(\mathrm{PF} 6)_{2}\right.$} & $\mathrm{NH}_{4} \mathrm{~S}_{2} \mathrm{O}_{8}$ & $\mathrm{MeOH} / \mathrm{DCM}$ & 12 & 0 \\
\hline G10 & {$\left[\mathrm{Ru}(\mathrm{dip})_{2}\right] \mathrm{Cl}_{2}$} & $\mathrm{NH}_{4} \mathrm{~S}_{2} \mathrm{O}_{8}$ & $\mathrm{MeOH} / \mathrm{DCM}$ & 9 & 0 \\
\hline G11 & {$\left[\operatorname{Ir}\left(\mathrm{dF}-\mathrm{CF}_{3}-\mathrm{ppy}\right)_{2}(\mathrm{dtbpy})\right] \mathrm{PF}_{6}$} & $\mathrm{NH}_{4} \mathrm{~S}_{2} \mathrm{O}_{8}$ & $\mathrm{MeOH} / \mathrm{DCM}$ & 48 & 0 \\
\hline G12 & $\left.\operatorname{Ir}(p p y)_{2}(d t b p y)\right] P F_{6}$ & $\mathrm{NH}_{4} \mathrm{~S}_{2} \mathrm{O}_{8}$ & $\mathrm{MeOH} / \mathrm{DCM}$ & 12 & 0 \\
\hline $\mathrm{H} 1$ & Mes-Acr & BPO & $\mathrm{MeOH} /$ Acetone & 57 & 0 \\
\hline $\mathrm{H} 2$ & {$\left[\mathrm{Ru}(\mathrm{bpz})_{3}\right]\left(\mathrm{PF}_{6}\right)_{2}$} & BPO & $\mathrm{MeOH} /$ Acetone & 7 & 0 \\
\hline $\mathrm{H} 3$ & {$\left[\mathrm{Ru}(\mathrm{bpy})_{3}(\mathrm{PF} 6)_{2}\right.$} & BPO & MeOH/Acetone & 19 & 0 \\
\hline $\mathrm{H} 4$ & {$\left[\mathrm{Ru}(\mathrm{dip})_{2}\right] \mathrm{Cl}_{2}$} & BPO & MeOH/Acetone & 13 & 0 \\
\hline $\mathrm{H} 5$ & {$\left[\operatorname{lr}\left(\mathrm{dF}-\mathrm{CF}_{3}-\mathrm{ppy}\right)_{2}(\mathrm{dtbpy})\right] \mathrm{PF}_{6}$} & BPO & MeOH/Acetone & 54 & 0 \\
\hline $\mathrm{H} 6$ & $\left.\operatorname{Ir}(p p y)_{2}(\mathrm{dtbpy})\right] \mathrm{PF}_{6}$ & BPO & $\mathrm{MeOH} /$ Acetone & 49 & 0 \\
\hline $\mathrm{H} 7$ & Mes-Acr & BPO & $\mathrm{MeOH} / \mathrm{DCM}$ & 84 & 0 \\
\hline $\mathrm{H} 8$ & {$\left[\mathrm{Ru}(\mathrm{bpz})_{3}\right]\left(\mathrm{PF}_{6}\right)_{2}$} & BPO & $\mathrm{MeOH} / \mathrm{DCM}$ & 4 & 0 \\
\hline $\mathrm{H} 9$ & {$\left[\mathrm{Ru}(\mathrm{bpy})_{3}(\mathrm{PF} 6)_{2}\right.$} & BPO & $\mathrm{MeOH} / \mathrm{DCM}$ & 15 & 0 \\
\hline $\mathrm{H} 10$ & {$\left[\mathrm{Ru}(\mathrm{dip})_{2}\right] \mathrm{Cl}_{2}$} & BPO & $\mathrm{MeOH} / \mathrm{DCM}$ & 3 & 0 \\
\hline $\mathrm{H} 11$ & {$\left[\operatorname{Ir}\left(\mathrm{dF}-\mathrm{CF}_{3}-\mathrm{ppy}\right)_{2}(\mathrm{dtbpy})\right] \mathrm{PF}_{6}$} & BPO & $\mathrm{MeOH} / \mathrm{DCM}$ & 83 & 0 \\
\hline $\mathrm{H} 12$ & $\left.\operatorname{Ir}(p p y)_{2}(\mathrm{dtbpy})\right] \mathrm{PF}_{6}$ & BPO & $\mathrm{MeOH} / \mathrm{DCM}$ & 75 & 0 \\
\hline
\end{tabular}


Figure S1. High-Throughput Analysis of Hydroxymethylation of Lepidine

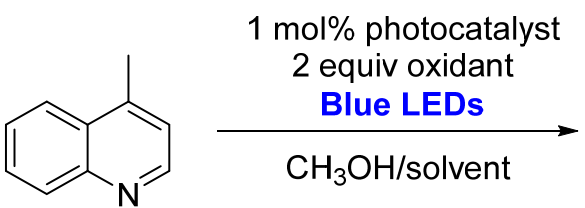

(1)<smiles>Cc1cc(CO)nc2ccccc12</smiles>

(1a)<smiles>Cc1cc(C)c2ccccc2n1</smiles>

(1b)

$\square \mathrm{CH}_{3} \mathrm{OH}$

$\square \mathrm{ACN}$

Acetone

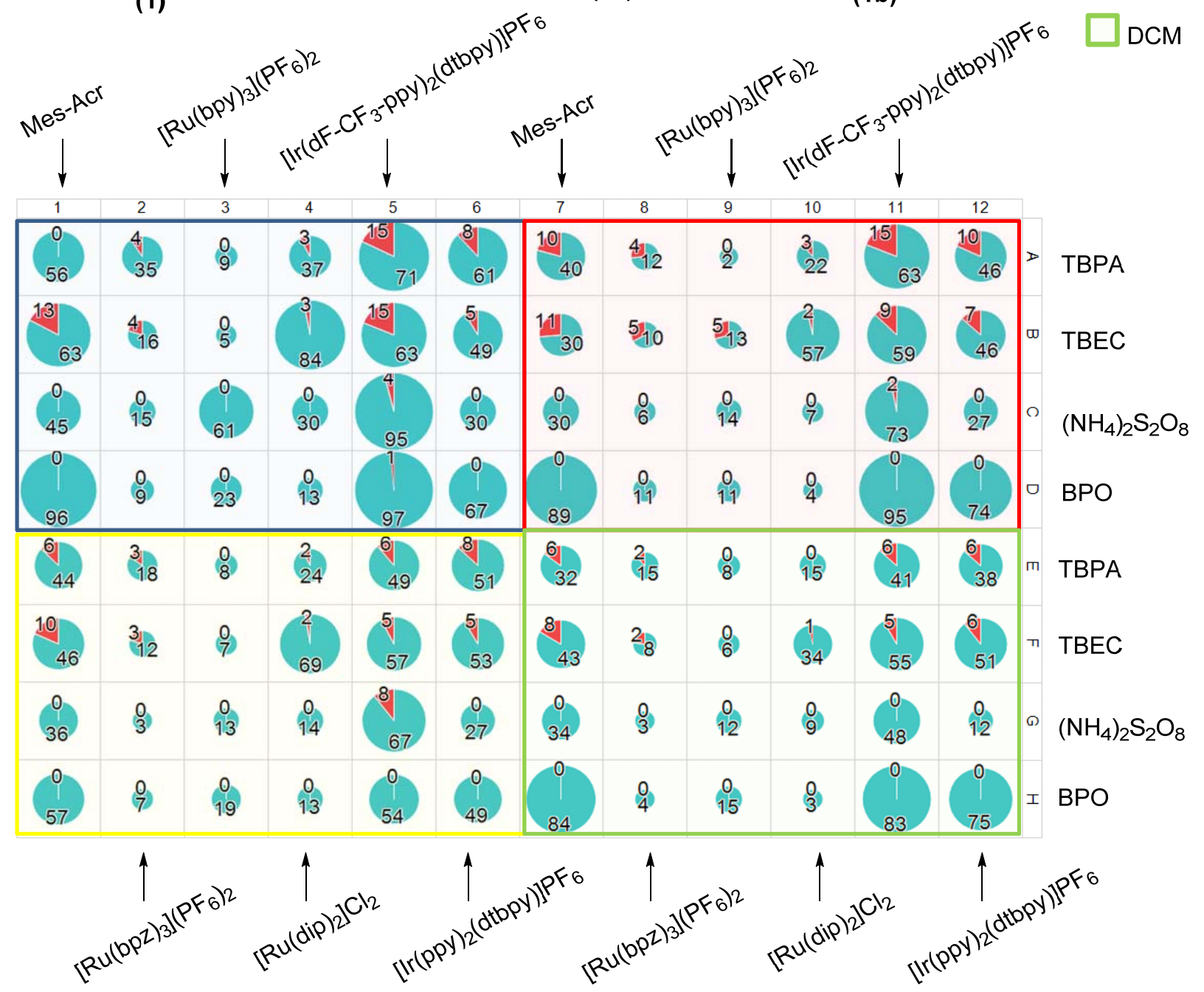




\section{B. Evaluation of Photoredox-Mediated and Minisci Hydroxymethylation}

Photoredox-mediated and literature reported Minisci hydroxymethylation conditions were compared as shown in Table S2. Procedure $B,{ }^{3} C,{ }^{4}$ and $D^{5}$ based off the corresponding cited literature examples.

Procedure A (Photoredox Method): In a $\mathrm{N}_{2}$-atmosphere glovebox, substrate $(0.175$ $\mathrm{mmol}), 85 \mathrm{mg}$ benzoyl peroxide $(0.350 \mathrm{mmol}, 2 \mathrm{eq}), 1.9 \mathrm{mg}\left[\mathrm{Ir}\left(\mathrm{dF}-\mathrm{CF}_{3}-\mathrm{ppy}\right)_{2}(\mathrm{dtbpy})\right] \mathrm{PF}_{6}$ $(1.75 \mu \mathrm{mol}, 0.01$ equiv.), $133 \mu \mathrm{L}$ TFA ( $1.75 \mathrm{mmol}, 10 \mathrm{eq})$, and $\mathrm{MeOH}(1.75 \mathrm{~mL}, 0.1 \mathrm{M})$ were added to a $4 \mathrm{~mL}$ vial with a pressure release septum (CG-4912-01) containing a teflon coated stirbar. The vial was then sealed and removed from the glovebox where it was placed in a compressed air cooled (to maintain $<30^{\circ} \mathrm{C}$ ) shallow mirrored glass vacuum dewar and illuminated with a $1.3 \mathrm{~A}$ blue LED lamp. Note: with substrates that are potentially light sensitive, a lower wattage LED array can be used $(5 \mathrm{~mA})$. The reaction was stirred at 600 RPM for 16 hours. Work up and purification details listed for individual compounds below. Photographs of the reaction manifold are available in the supporting information of our previous publication. ${ }^{2}$

Procedure B: Substrate $(0.175 \mathrm{mmol}), 80 \mathrm{mg} \mathrm{NH} \mathrm{S}_{2} \mathrm{O}_{8}(0.350 \mathrm{mmol}, 2 \mathrm{eq}), 9.3 \mu \mathrm{L}$ $\mathrm{H}_{2} \mathrm{SO}_{4}(0.175 \mathrm{mmol}, 1 \mathrm{eq})$, and $390 \mu \mathrm{CH}_{3} \mathrm{OH}: \mathrm{H}_{2} \mathrm{O}(2: 1)$ were added to a $4 \mathrm{~mL}$ vial with a pressure release septum (CG-4912-01) containing a teflon coated stirbar. The vial was then sealed and heated at $70{ }^{\circ} \mathrm{C}$ for $24 \mathrm{~h}$. The $\mathrm{CH}_{3} \mathrm{OH}$ was removed in vacuo and $\mathrm{CH}_{2} \mathrm{Cl}_{2}(5 \mathrm{~mL})$ and saturated sodium bicarbonate $(3 \mathrm{~mL})$ were added. The organic layer was then dried over $\mathrm{Na}_{2} \mathrm{SO}_{4}$ and the crude mixture was purified as listed below for each compound.

Procedure C: Substrate $(0.175 \mathrm{mmol}), 112 \mathrm{mg} \mathrm{NH}_{4} \mathrm{~S}_{2} \mathrm{O}_{8}(0.490 \mathrm{mmol}, 2.8 \mathrm{eq}), 4.2 \mathrm{mg}$ $\mathrm{AgNO}_{3}(0.0263 \mathrm{mmol}, 15 \mathrm{~mol} \%), 6.5 \mu \mathrm{L} \mathrm{H}_{2} \mathrm{SO}_{4}(0.123 \mathrm{mmol}, 0.7 \mathrm{eq}), 1.5 \mathrm{~mL} \mathrm{CH} \mathrm{CH}_{3} \mathrm{OH}$ were added to a $4 \mathrm{~mL}$ vial with a pressure release septum (CG-4912-01) containing a teflon coated stirbar. The vial was then sealed and heated at $70{ }^{\circ} \mathrm{C}$ for $1.5 \mathrm{~h}$. The reaction solvent was removed in vacuo and the residue redissolved in $\mathrm{CH}_{2} \mathrm{Cl}_{2}(5 \mathrm{~mL})$. The organic layer was then washed with saturated sodium bicarbonate, and dried over $\mathrm{Na}_{2} \mathrm{SO}_{4}$. Purification details listed for individual compounds below.

Procedure D: Substrate $(0.175 \mathrm{mmol}), 160 \mathrm{mg} \mathrm{NH}{ }_{4} \mathrm{~S}_{2} \mathrm{O}_{8}(0.7 \mathrm{mmol}, 4 \mathrm{eq}), 3 \mathrm{mg} \mathrm{AgNO}$ (0.0175 mmol, $10 \mathrm{~mol} \%), 13 \mu \mathrm{L}$ TFA $(0.175,1 \mathrm{eq}), 1.3 \mathrm{~mL} \mathrm{CH} \mathrm{CH}_{3} \mathrm{O} \mathrm{H}_{2} \mathrm{O}(1: 1)$ were added to a $4 \mathrm{~mL}$ vial with a pressure release septum (CG-4912-01) containing a teflon coated stirbar. The vial was then sealed and heated at $70{ }^{\circ} \mathrm{C}$ for $24 \mathrm{~h}$. The $\mathrm{CH}_{3} \mathrm{OH}$ was removed in vacuo and $\mathrm{CH}_{2} \mathrm{Cl}_{2}(5 \mathrm{~mL})$ and saturated sodium bicarbonate $(3 \mathrm{~mL})$ were added. The organic layer was then dried over $\mathrm{Na}_{2} \mathrm{SO}_{4}$ and the crude mixture was purified as listed below for each compound. 
Table S2. Comparison Photoredox-Mediated Conditions with Minisci Conditions for the Hydroxymethylation of Substrates 1-3

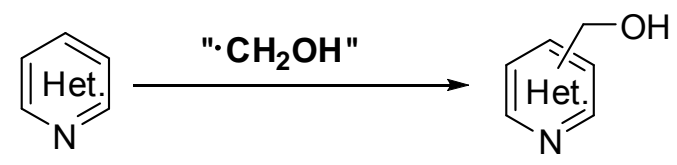

\begin{tabular}{|c|c|c|c|c|}
\hline Entry & Substrate & Procedure & \%Conversion $^{\mathbf{a}}$ & $\begin{array}{c}\text { Major Product } \\
\text { (\% Isolated Yield) }\end{array}$ \\
\hline 1 & $\mathbf{1}$ & $\mathrm{A}$ & $100 \%$ & $\mathbf{1 a}(81 \%)$ \\
\hline 2 & $\mathbf{1}$ & $\mathrm{B}$ & $80 \%$ & $\mathbf{1 a}(67 \%)$ \\
\hline 3 & $\mathbf{1}$ & $\mathrm{C}$ & $25 \%$ & $\mathbf{1 a}(16 \%)$ \\
\hline 4 & $\mathbf{1}$ & $\mathrm{D}$ & $100 \%$ & $\mathbf{1 a}(56 \%)$ \\
\hline 5 & $\mathbf{2}$ & $\mathrm{A}$ & $100 \%$ & $\mathbf{2 a}(60 \%)$ \\
\hline 6 & $\mathbf{2}$ & $\mathrm{B}$ & $80 \%$ & $\mathbf{2 a}(8 \%)$ \\
\hline 7 & $\mathbf{3}$ & $\mathrm{A}$ & $80 \%$ & $\mathbf{3 a}(32 \%)$ \\
\hline 8 & $\mathbf{3}$ & $\mathrm{B}$ & $100 \%$ & $\mathbf{3 b}(66 \%)$ \\
\hline 9 & $\mathbf{3}$ & $\mathrm{C}$ & $100 \%$ & $\mathbf{3 b}(24 \%)$ \\
\hline
\end{tabular}

${ }^{a}$ Percent conversion approximation based on relative HPLC peak area integrations of starting material compared with product. 


\section{Evaluation of Lewis Acids and Oxidants for the Hydroxymethylation Reaction}

Combinations of $\mathrm{BPO}$ or $\mathrm{NH}_{4} \mathrm{~S}_{2} \mathrm{O}_{8}$ and TFA or $\mathrm{BF}_{3} \mathrm{OEt}_{2}$ were tested on three substrates as shown in Table S3. A combination of $\mathrm{NH}_{4} \mathrm{~S}_{2} \mathrm{O}_{8} / \mathrm{BF}_{3} \mathrm{OEt}_{2}$ was superior to the standard condtions only for substrate 13 (entry 4).

Table S3. Summary of Acid and Oxidant Evaluation

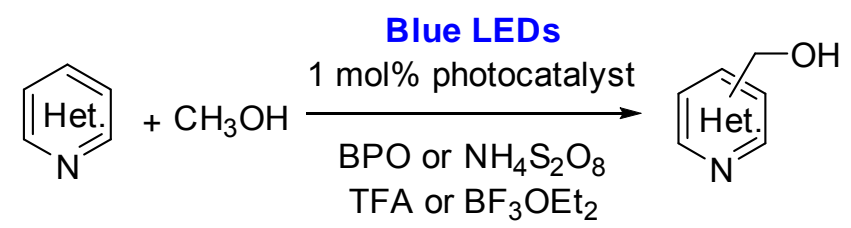

\begin{tabular}{|c|c|c|c|c|}
\hline Entry $^{a}$ & Substrate & Oxidant/Acid & Product & \%Yield \\
\hline 1 & 13 & BPO/TFA & $13 a$ & $17 \%^{b}$ \\
\hline 2 & 13 & $\mathrm{BPO} / \mathrm{BF}_{3} \mathrm{OEt}_{2}$ & $13 a$ & $18 \%^{b}$ \\
\hline 3 & 13 & $\mathrm{NH}_{4} \mathrm{~S}_{2} \mathrm{O}_{8} / \mathrm{TFA}$ & $13 a$ & $20 \%^{b}$ \\
\hline 4 & 13 & $\mathrm{NH}_{4} \mathrm{~S}_{2} \mathrm{O}_{8} / \mathrm{BF}_{3} \mathrm{OEt}_{2}$ & $13 \mathbf{a}$ & $28 \%^{b}$ \\
\hline 5 & 11 & $\mathrm{BPO} / \mathrm{TFA}$ & $11 a$ & $26 \%^{c}$ \\
\hline 6 & 11 & $\mathrm{BPO} / \mathrm{BF}_{3} \mathrm{OEt}_{2}$ & $11 a$ & $22 \%^{c}$ \\
\hline 7 & 11 & $\mathrm{NH}_{4} \mathrm{~S}_{2} \mathrm{O}_{8} / \mathrm{BF}_{3} \mathrm{OEt}_{2}$ & $11 a$ & $17 \%^{c}$ \\
\hline 8 & 20 & BPO/TFA & $20 a$ & $17 \%^{c}$ \\
\hline 9 & 20 & $\mathrm{NH}_{4} \mathrm{~S}_{2} \mathrm{O}_{8} / \mathrm{BF}_{3} \mathrm{OEt}_{2}$ & $20 a$ & $\mathrm{ND}^{c}$ \\
\hline
\end{tabular}

${ }^{a}$ Conditions: Substrate (1 eq), BPO or $\mathrm{NH}_{4} \mathrm{~S}_{2} \mathrm{O}_{3}$ (2 eq), [Ir(dF-CF - -ppy) $\left.)_{2}(\mathrm{dtbpy})\right] \mathrm{PF}_{6}$ (1 mol\%), TFA (10 eq) or $\mathrm{BF}_{3} \mathrm{OEt}_{2}(3 \mathrm{eq}), \mathrm{MeOH}(0.1 \mathrm{M}), 8-16$ hours, $\mathrm{rt}^{\circ}{ }^{b}$ Isolated yield; ${ }^{\mathrm{c}}$ Yield approximated by comparing the UV response obtained by UPLC of starting material and potential product; ND = not detected.

\section{References}

1. Yu, W.-Y.; Sit, Q. N.; Zhou, Z.; Chang, A. S.-C. Org. Lett., 2009, 11, 3174.

2. See supporting info: DiRocco, D. A.; Dykstra, K.; Krska, S.; Vachal, P.; Conway, D. V.; Tudge, M. Angew. Chem. Int. Ed. 2014, 53, 4802.

3. Buratti, W.; Gardini, G. P. Minisci, F. Tetrahedron, 1971, 27, 3655.

4. Nam, T.-G., Rector, C. L.; Kim, H.-Y.; Sonnen, A. F.-P.; Meyer, R.; Nau, W. M.; Atkinson, J.; Rintoul, J.; Pratt, D. A.; Porter, N. A. J. Am. Chem. Soc., 2007, 129, 10211.

5. Minisci, F.; Porta, O.; Recupero, F.; Punta, C.; Gambarotti, C.; Pruna, B.; Pierini, M.; Fontanta, F. Synlett, 2004, 5, 874. 
${ }^{1} \mathrm{H}$ NMR spectrum of $1 \mathbf{a}\left(500 \mathrm{MHz}, \mathrm{CD}_{3} \mathrm{OD}\right)$

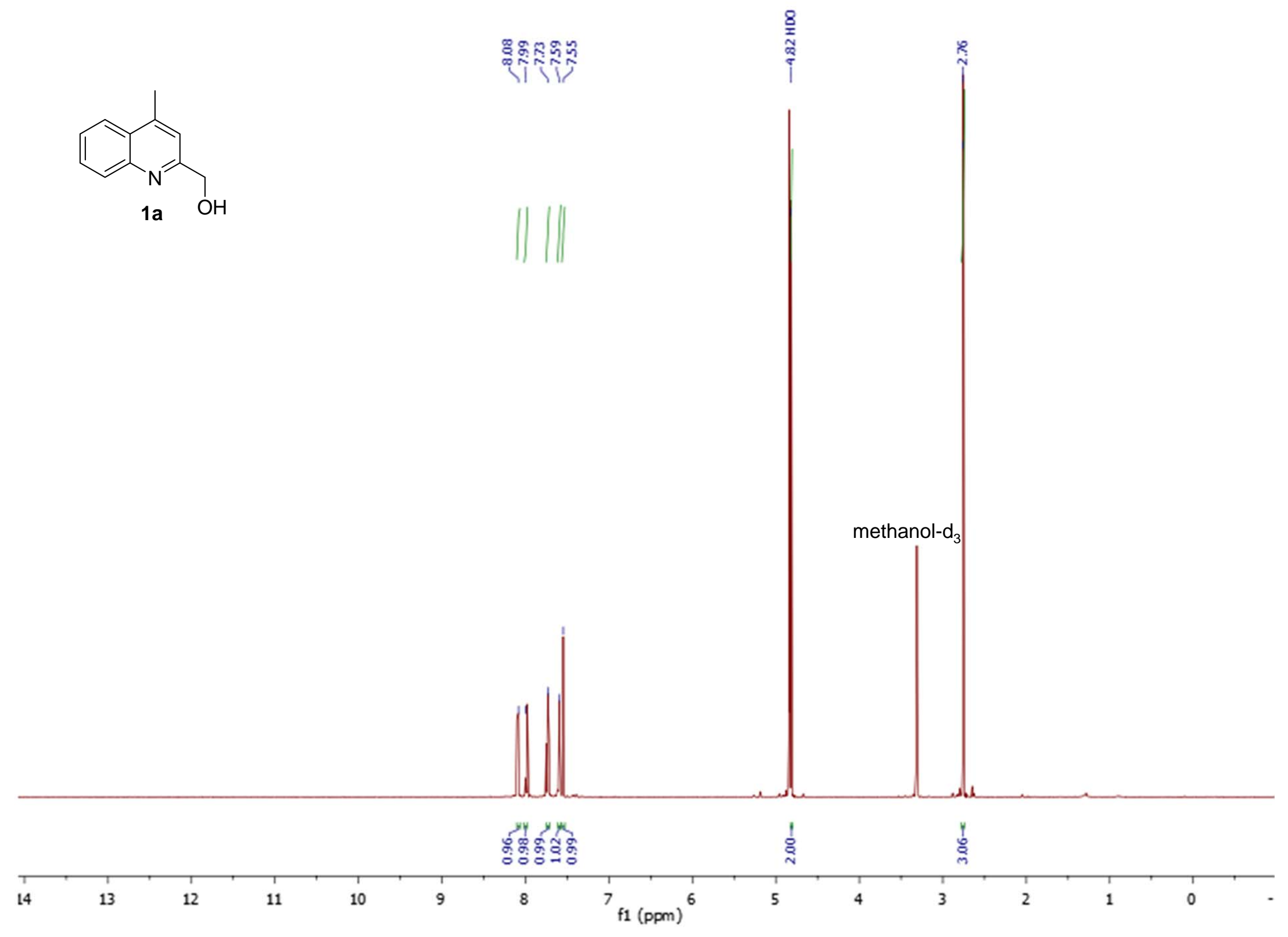


${ }^{13} \mathrm{C}$ NMR spectrum of $1 \mathrm{a}\left(125 \mathrm{MHz}, \mathrm{CD}_{3} \mathrm{OD}\right)$

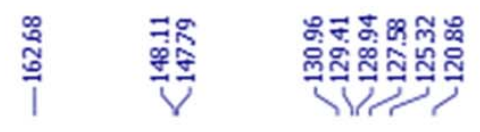
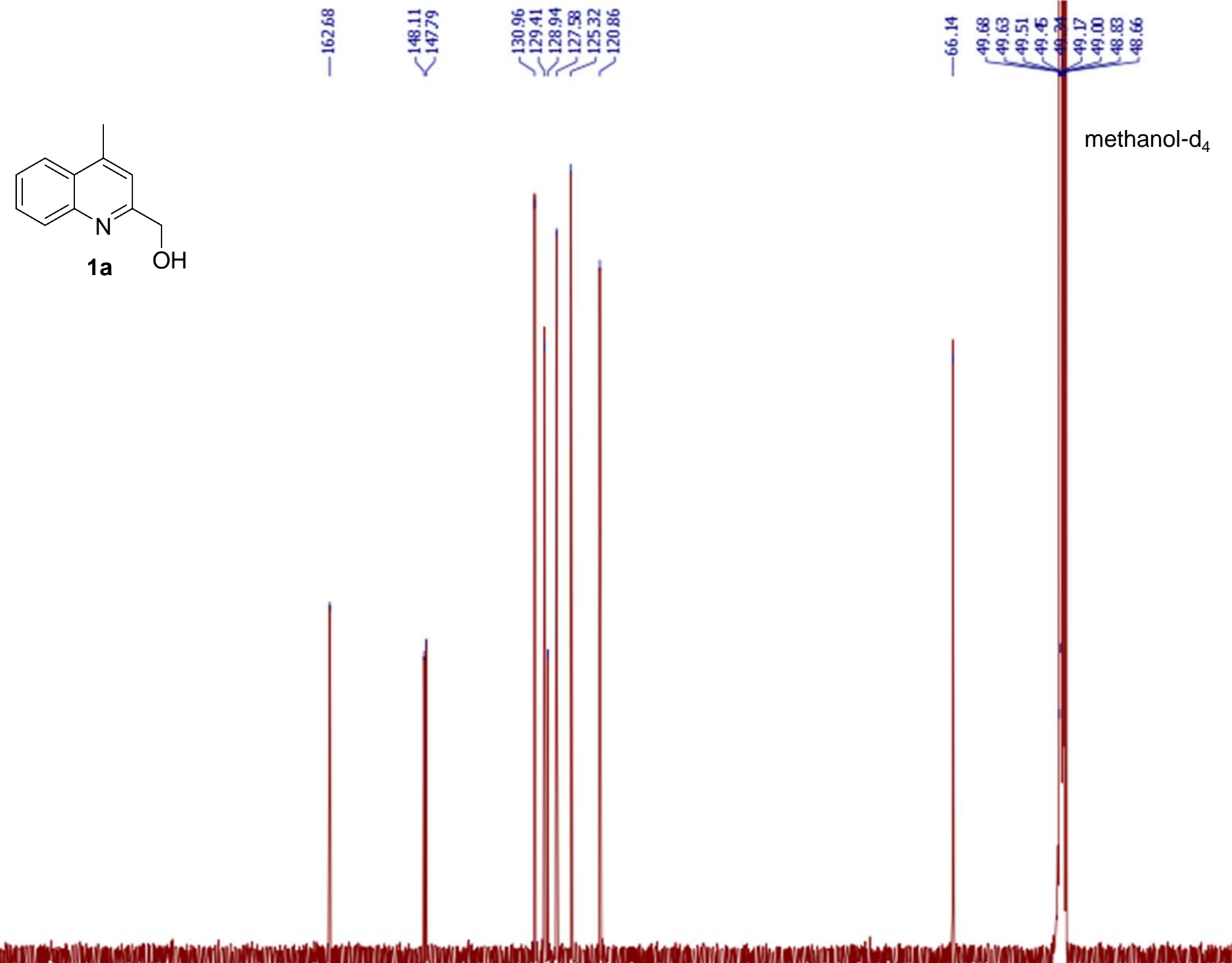

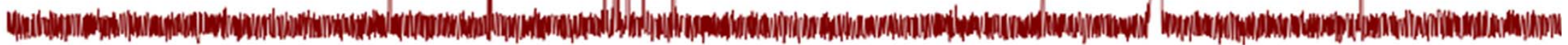

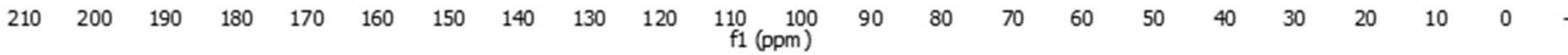




\section{${ }^{1} \mathrm{H}$ NMR spectrum of $\mathbf{2 a} \cdot T F A\left(600 \mathrm{MHz}, \mathrm{CD}_{\beta} \mathrm{OD}\right)$}

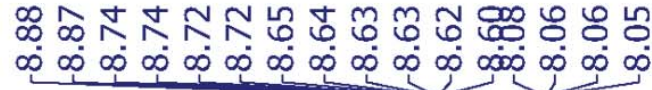

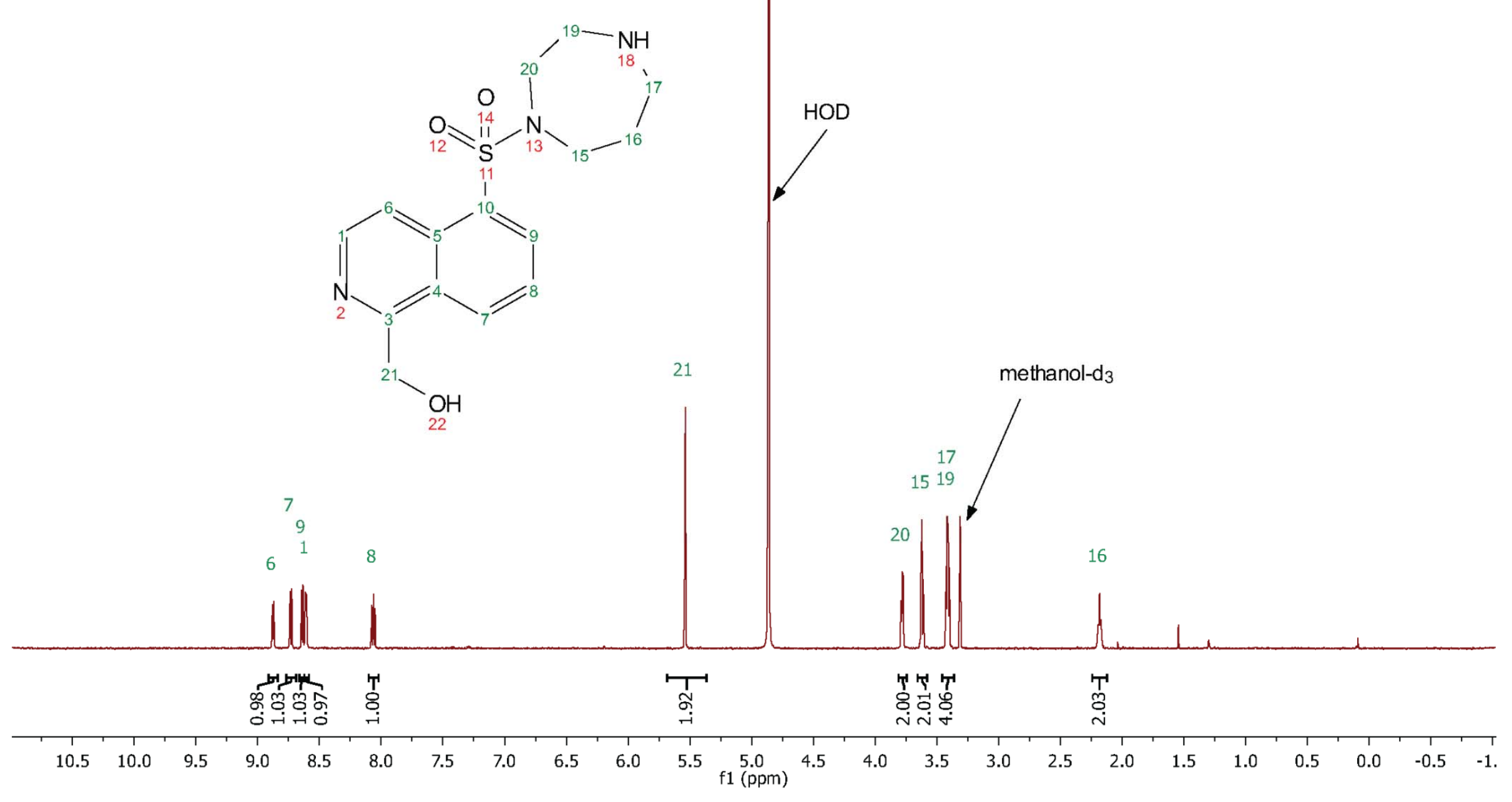

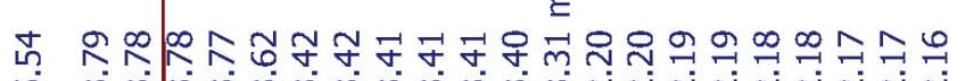

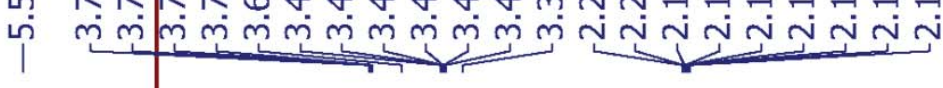


${ }^{13} \mathrm{C}$ NMR spectrum of 2a•TFA (150 MHz, $\left.\mathrm{CD}_{3} \mathrm{OD}\right)$
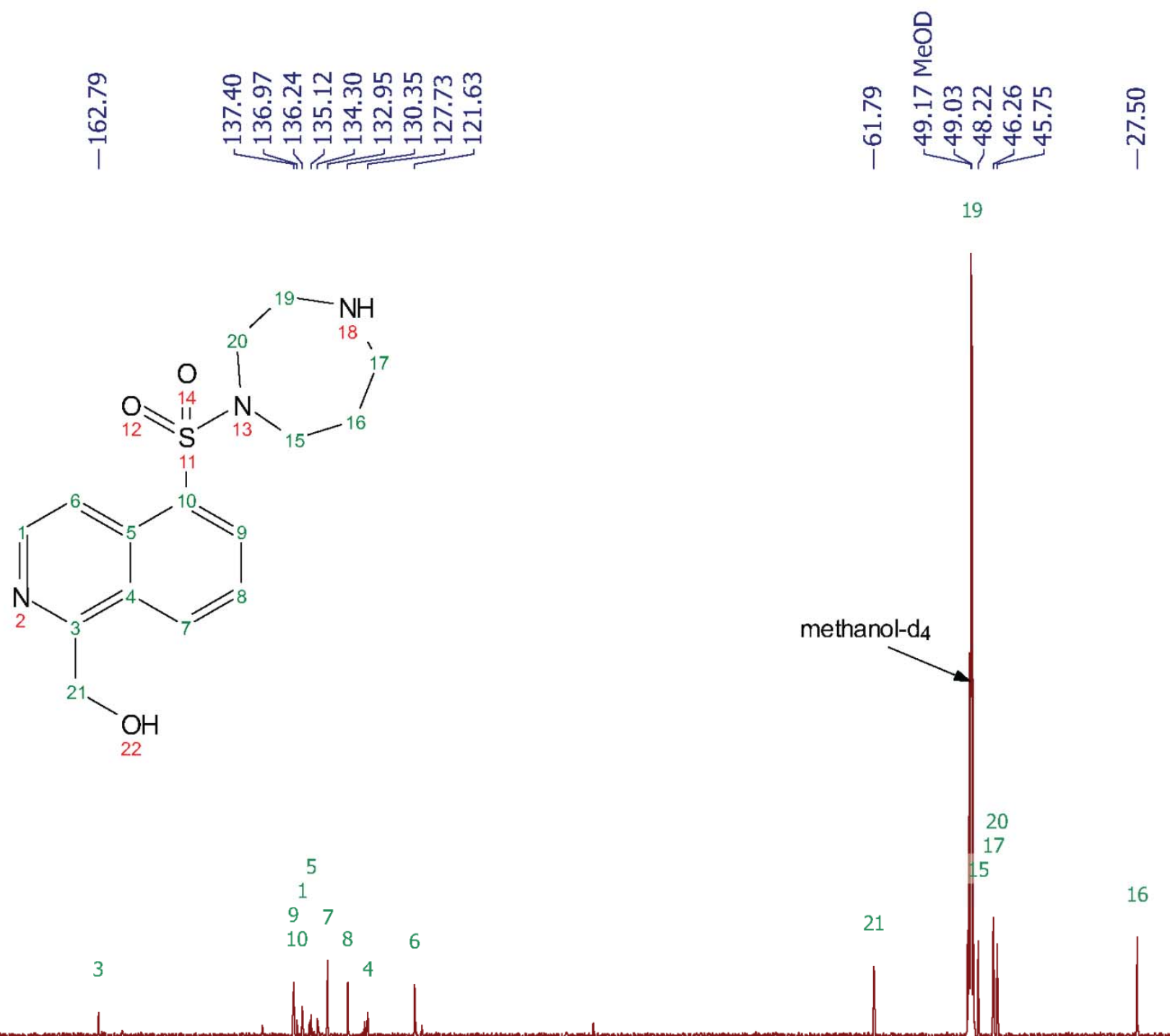

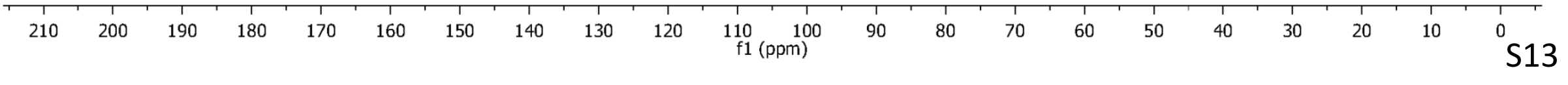


${ }^{1} \mathrm{H} /{ }^{1} \mathrm{H}$ COSY NMR spectrum of 2a•TFA (600/600 MHz, CD $\left.{ }_{3} \mathrm{OD}\right)$

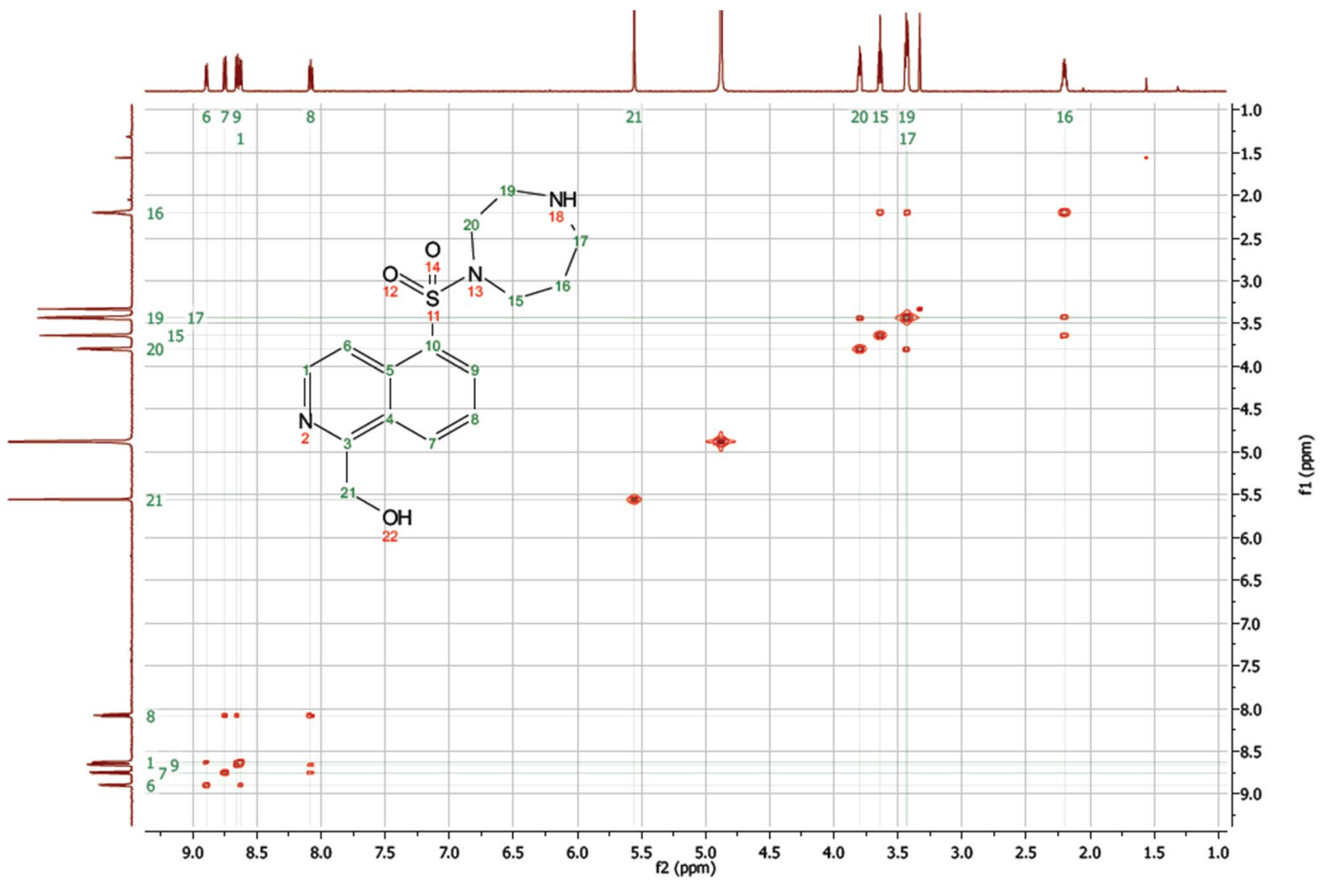


${ }^{1} \mathrm{H} /{ }^{13} \mathrm{C}$ HSQC NMR spectrum of 2a.TFA (600/ $\left.150 \mathrm{MHz}, \mathrm{CD}_{3} \mathrm{OD}\right)$

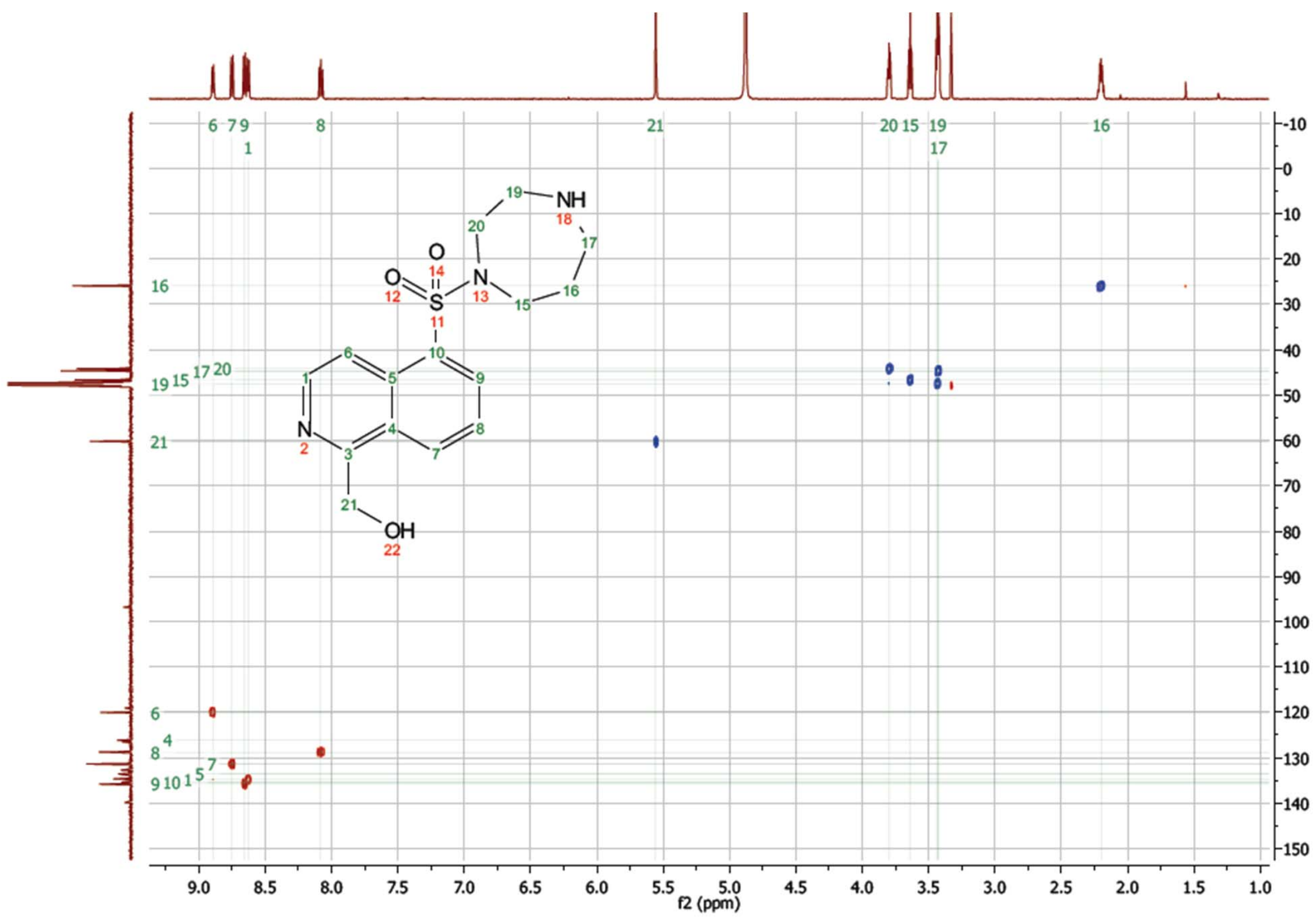


${ }^{1} \mathrm{H} /{ }^{13} \mathrm{C}$ HMBC NMR spectrum of 2a・TFA (600 / $\left.150 \mathrm{MHz}, \mathrm{CD}_{3} \mathrm{OD}\right)$

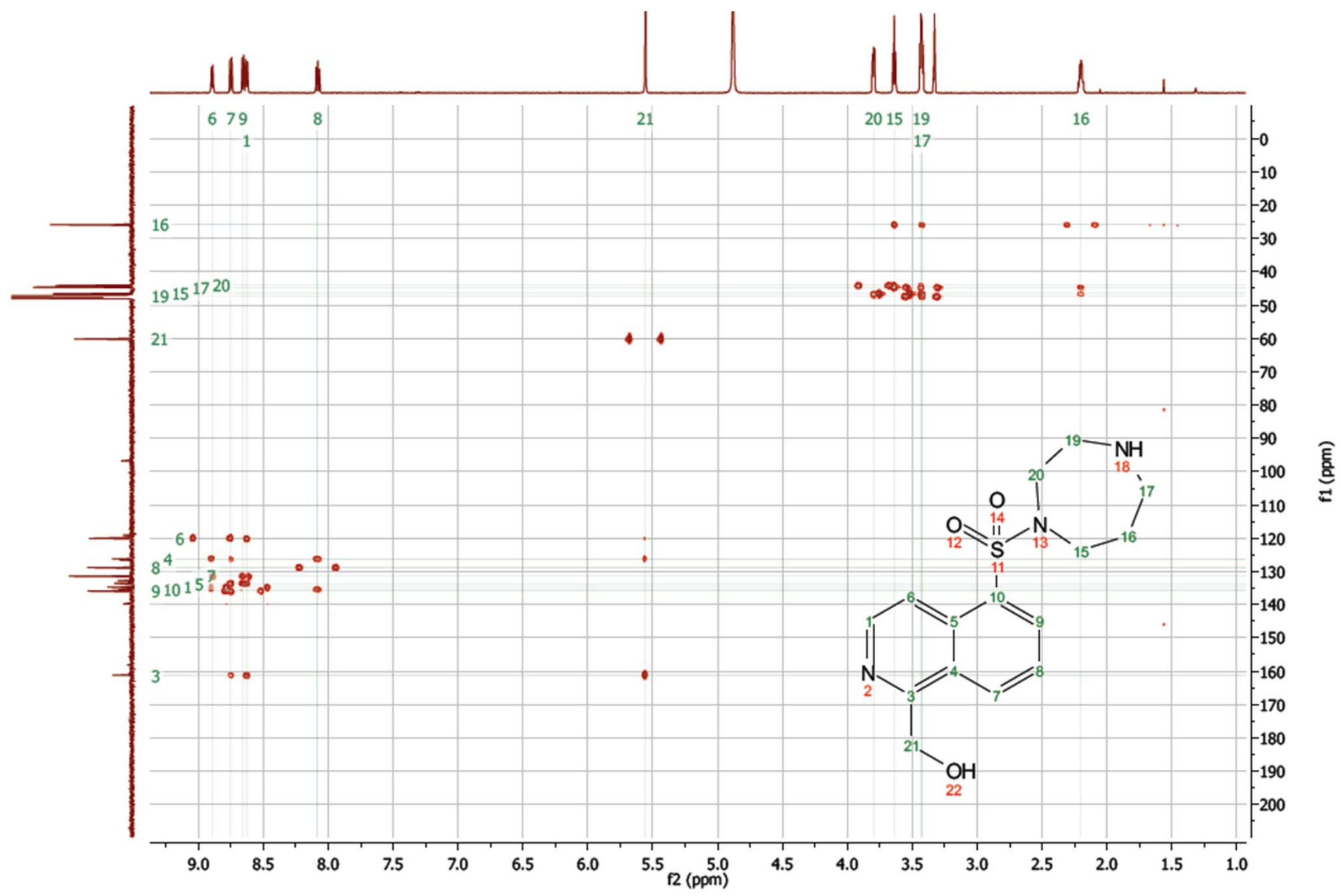


${ }^{1} \mathrm{H} /{ }^{1} \mathrm{H}$ ROESY NMR spectrum of 2a・TFA (600/600 MHz, CD $\left.{ }_{3} \mathrm{OD}\right)$

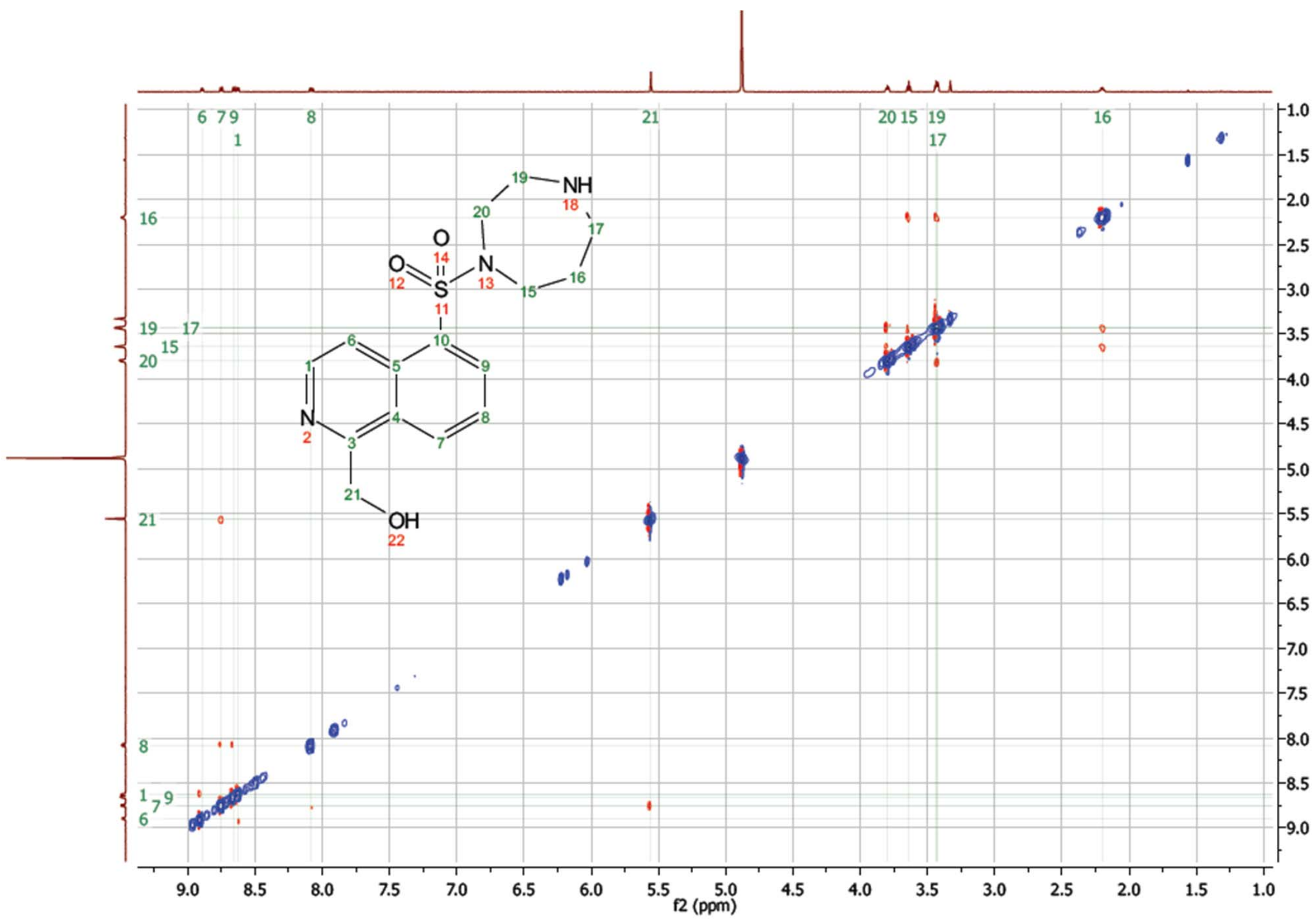

틀 
${ }^{1} \mathrm{H} /{ }^{15} \mathrm{~N}$ HMBC NMR spectrum of 2a•TFA (600 / $\left.61 \mathrm{MHz}, \mathrm{CD}_{3} \mathrm{OD}\right)$

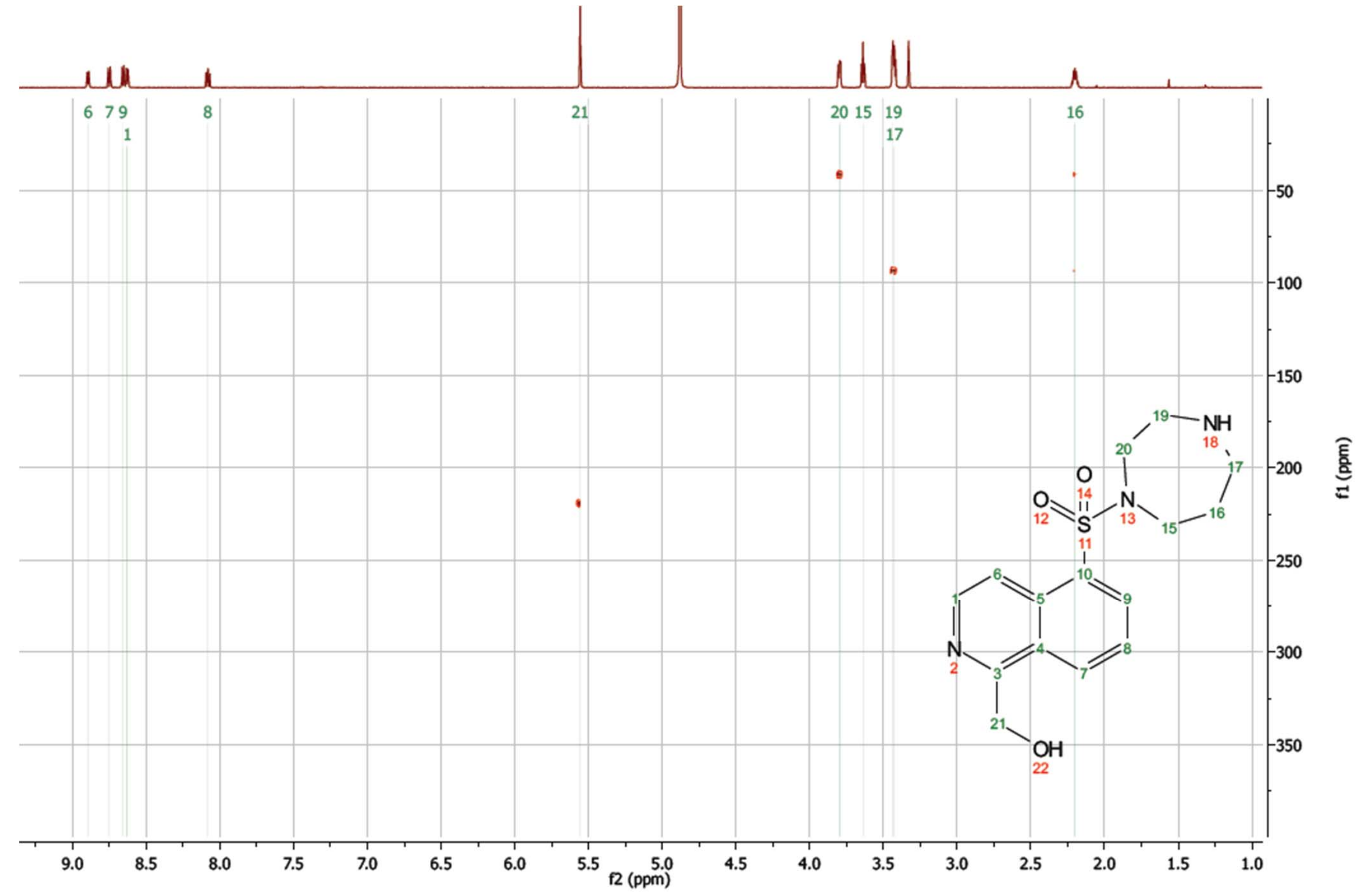


${ }^{1} \mathrm{H}$ NMR spectrum of $\mathbf{3 a}\left(600 \mathrm{MHz}, \mathrm{DMSO}-\mathrm{d}_{6}\right)$

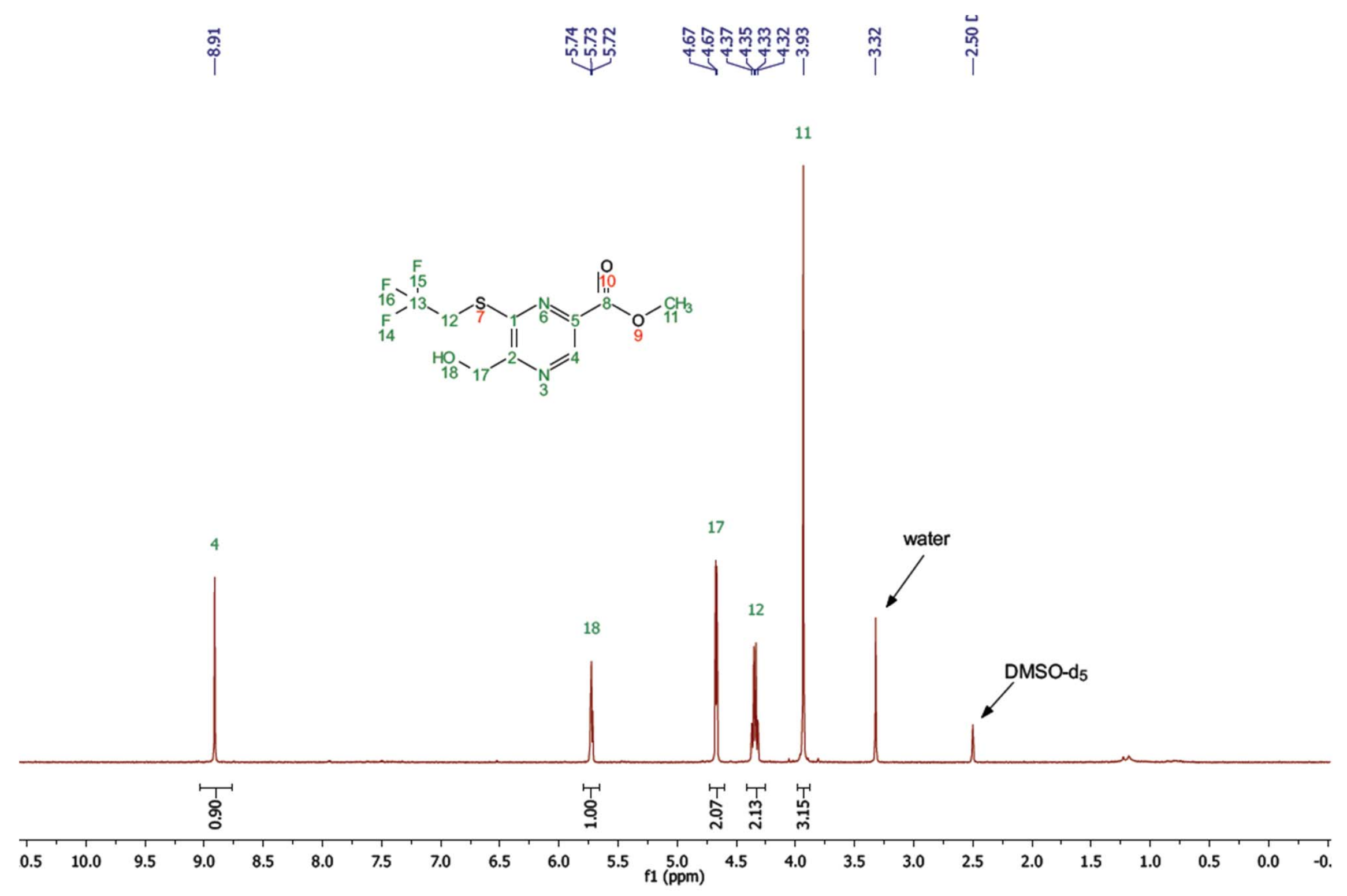


${ }^{13} \mathrm{C}$ NMR spectrum of $\mathbf{3 a}\left(150 \mathrm{MHz}, \mathrm{DMSO}-\mathrm{d}_{6}\right)$

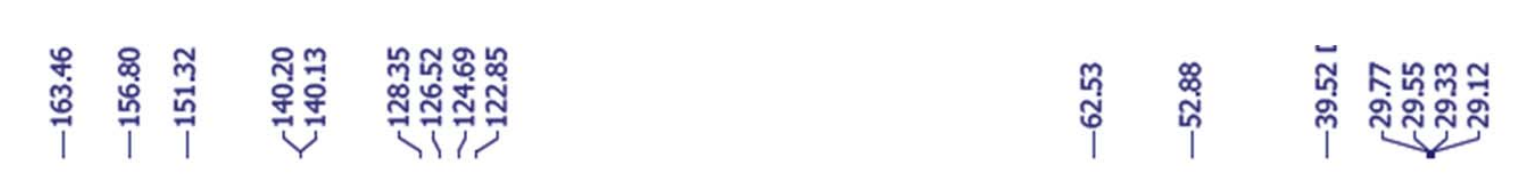
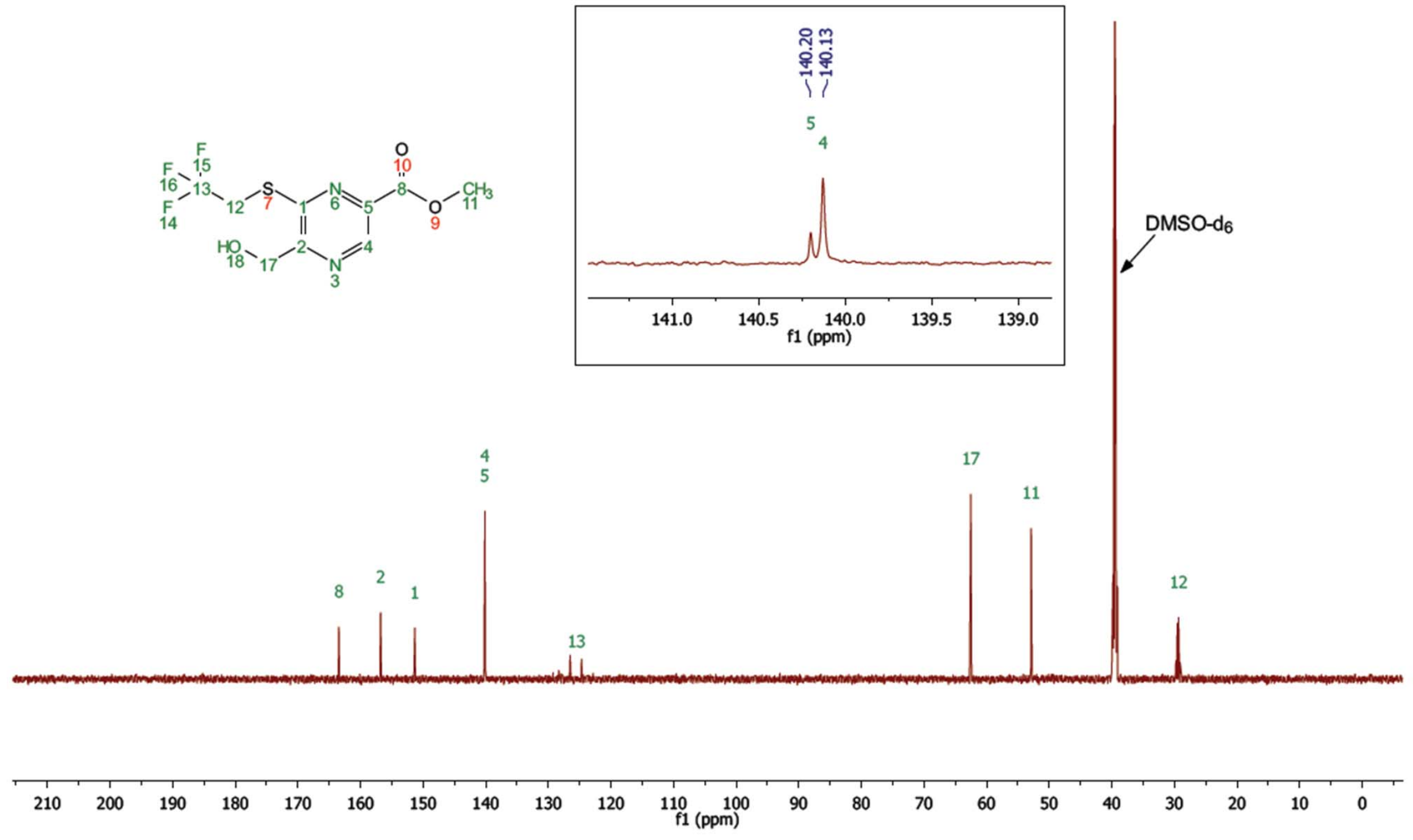
${ }^{19}$ F NMR spectrum of $3 \mathbf{a}\left(564 \mathrm{MHz}, \mathrm{DMSO}-\mathrm{d}_{6}\right)$

${ }^{19} \mathrm{~F}$ NMR (564 MHz, DMSO-d6)

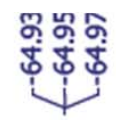

Sample: 0363972-0136C

$14,15,16$
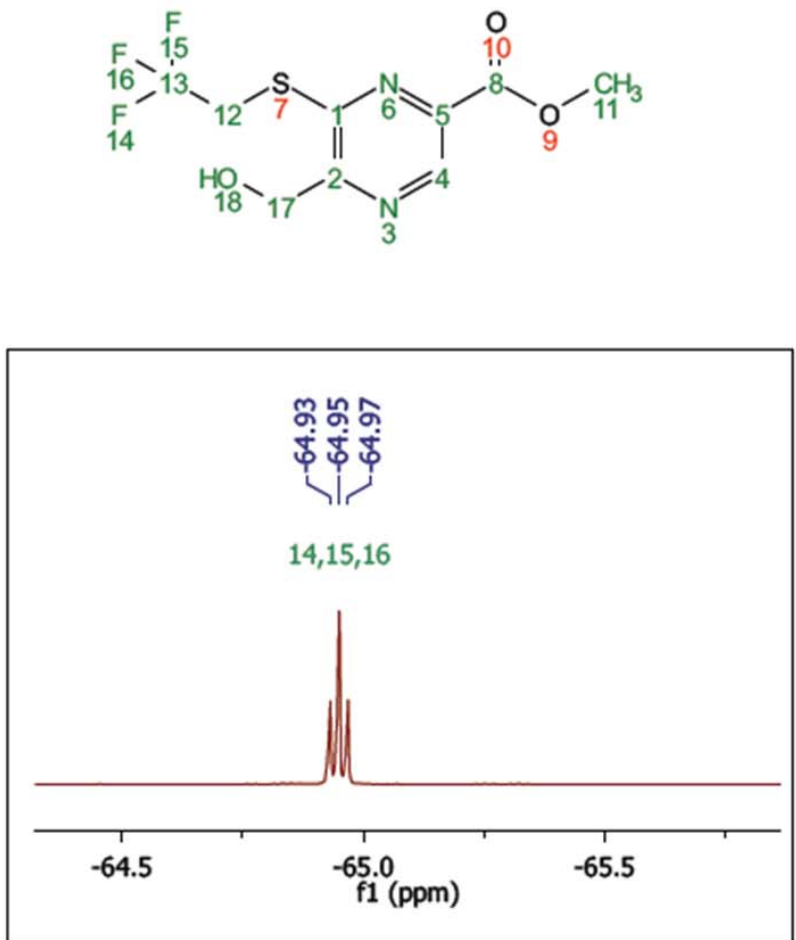

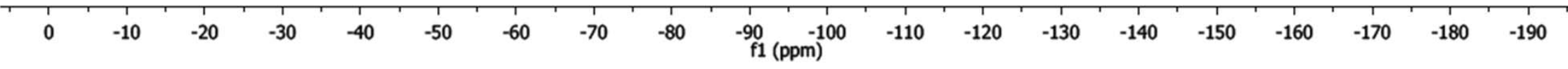


${ }^{1} \mathrm{H} /{ }^{1} \mathrm{H}$ COSY NMR spectrum of $3 \mathbf{a}\left(600 / 600 \mathrm{MHz}\right.$, DMSO-d $\left.\mathrm{d}_{6}\right)$

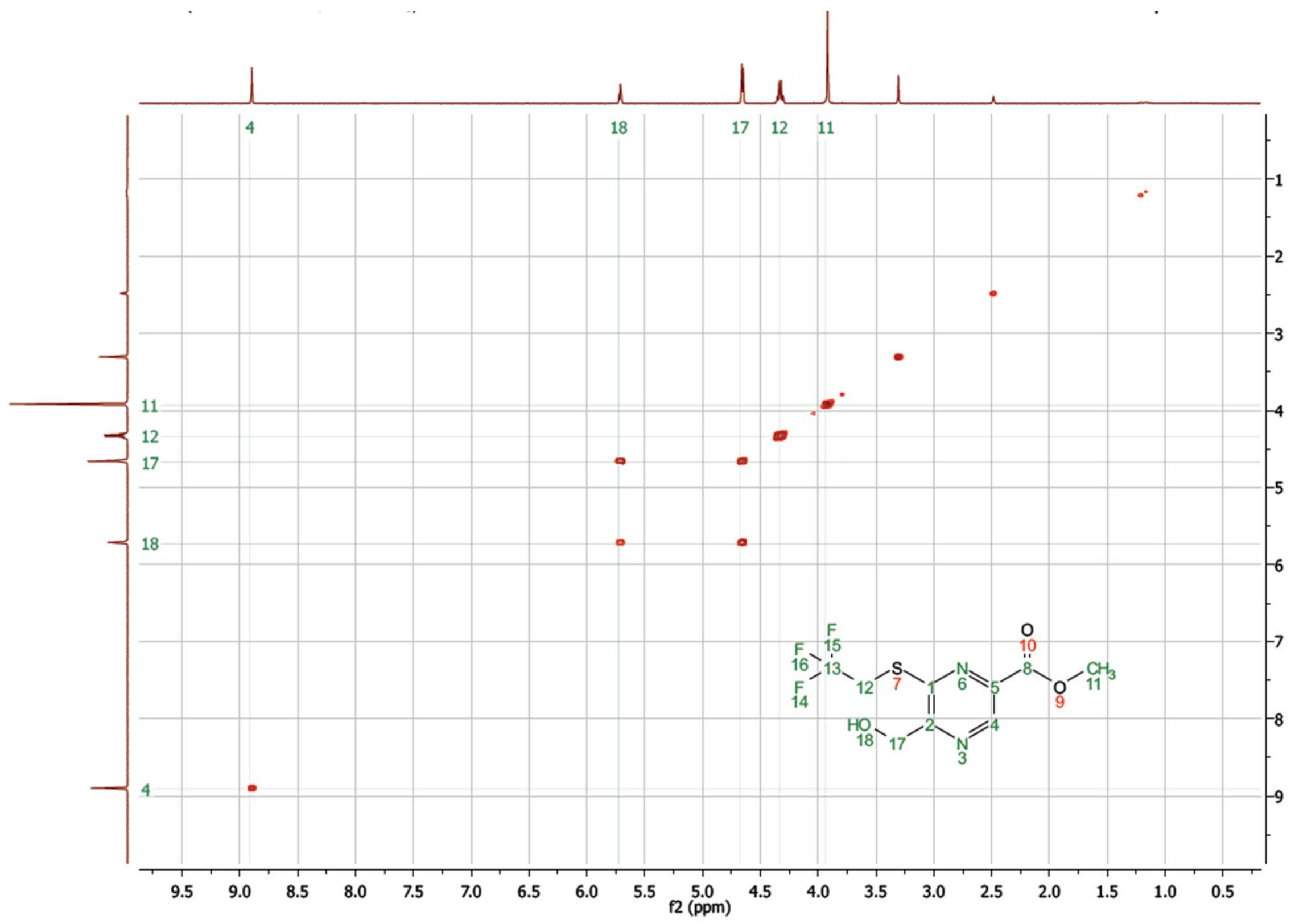


${ }^{1} \mathrm{H} /{ }^{13} \mathrm{C}$ HSQC NMR spectrum of $3 a\left(600 / 150 \mathrm{MHz}\right.$, DMSO-d $\left.\mathrm{d}_{6}\right)$

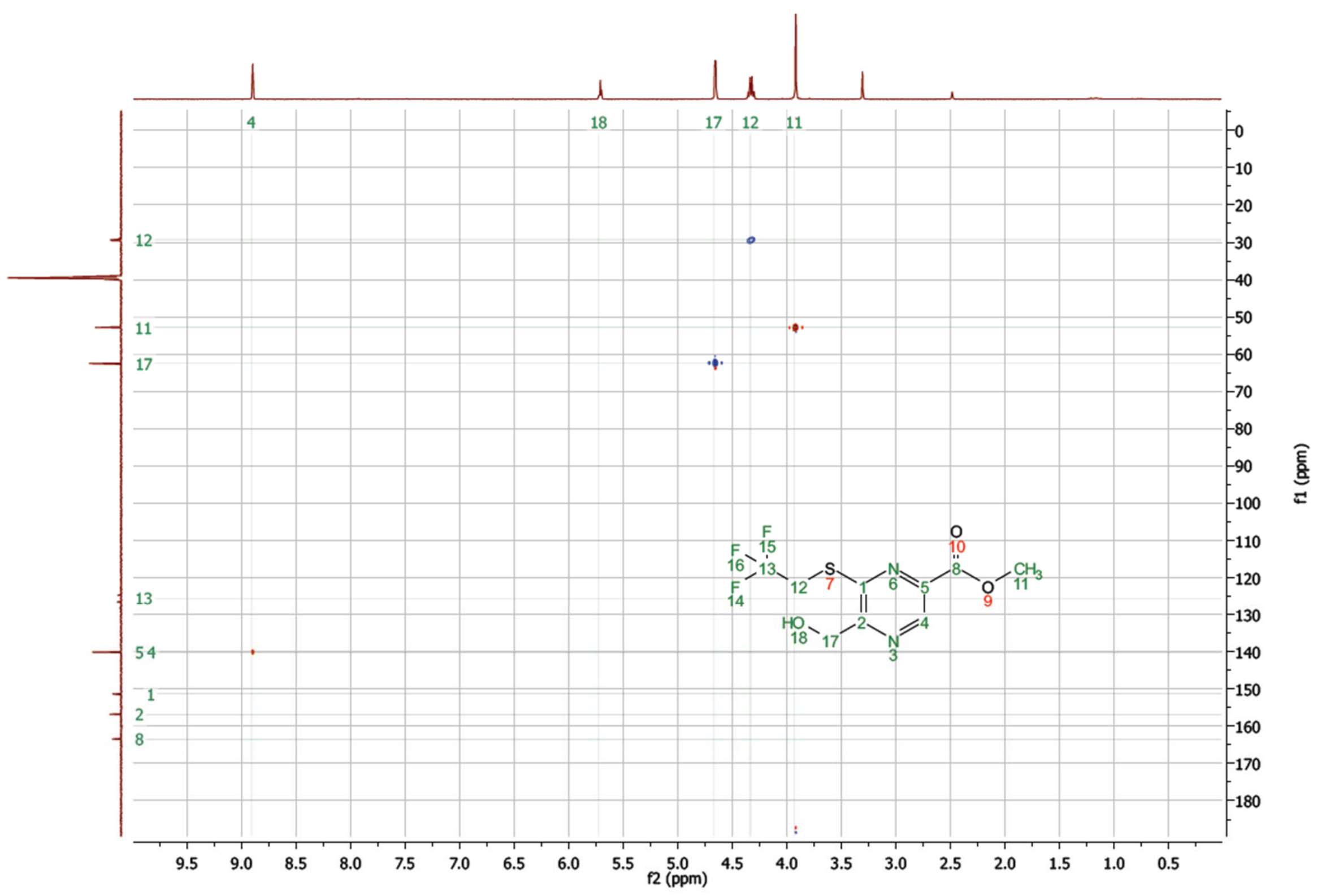


${ }^{1} \mathrm{H} /{ }^{13} \mathrm{C}$ HMBC NMR spectrum of $\mathbf{3 a}\left(600 / 150 \mathrm{MHz}\right.$, DMSO- $\left.\mathrm{d}_{6}\right)$

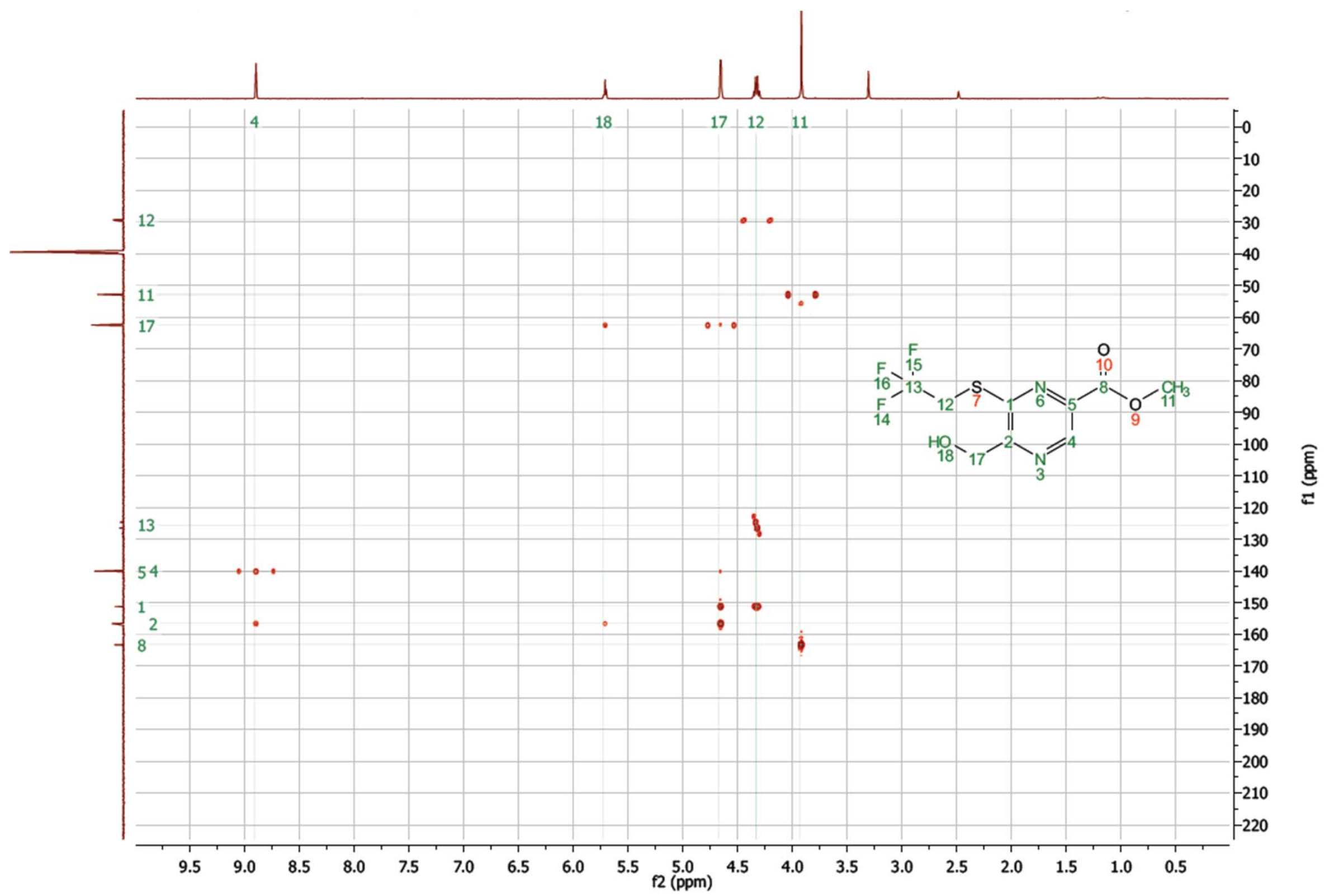


${ }^{1} \mathrm{H} /{ }^{1} \mathrm{H}$ ROESY NMR spectrum of $\mathbf{3 a}\left(600 / 600 \mathrm{MHz}\right.$, DMSO- $\left.\mathrm{d}_{6}\right)$

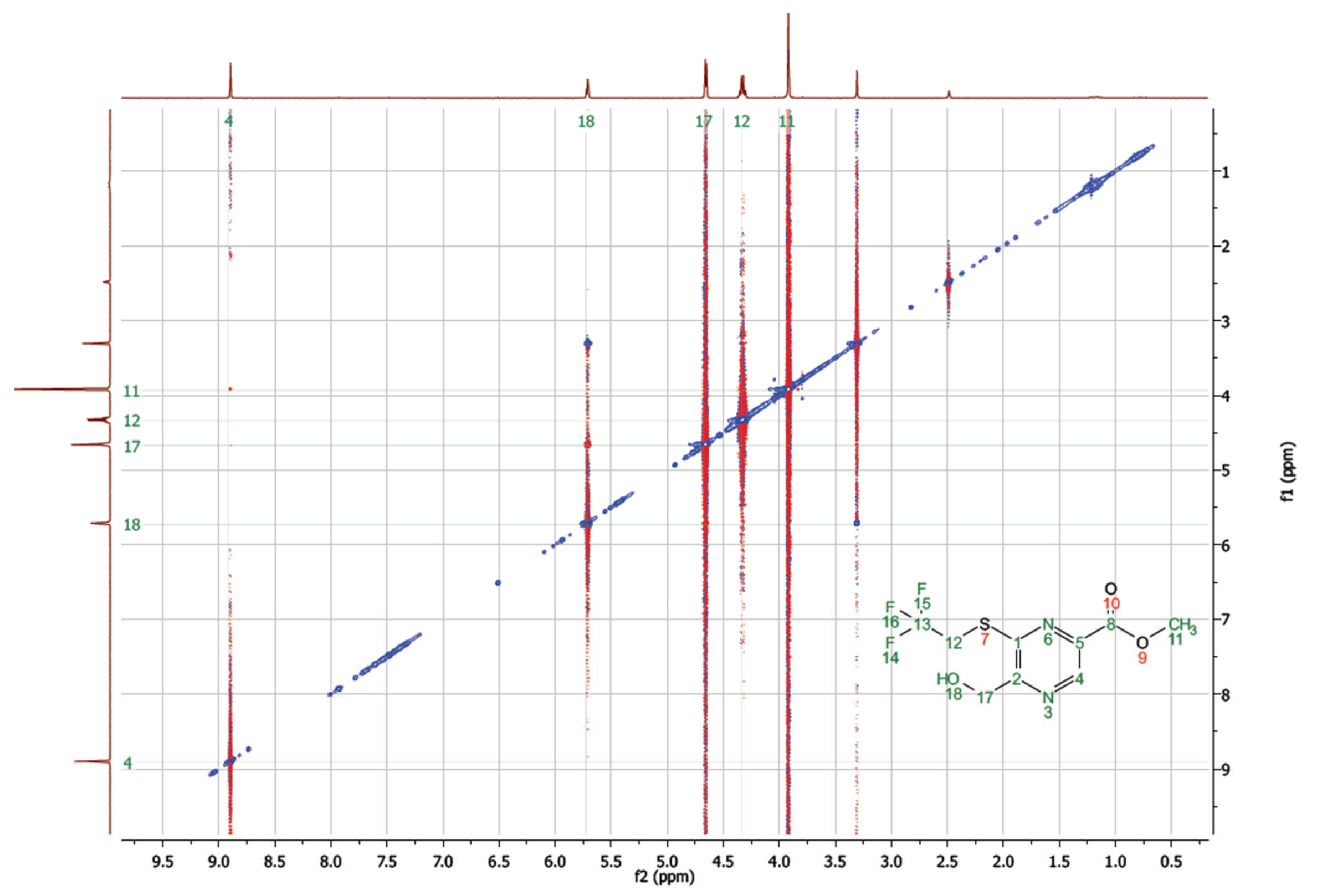


${ }^{1} \mathrm{H} /{ }^{15} \mathrm{~N} \mathrm{HMBC}$ NMR spectrum of $3 \mathbf{3}\left(600 / 61 \mathrm{MHz}\right.$, DMSO- $\left.\mathrm{d}_{6}\right)$

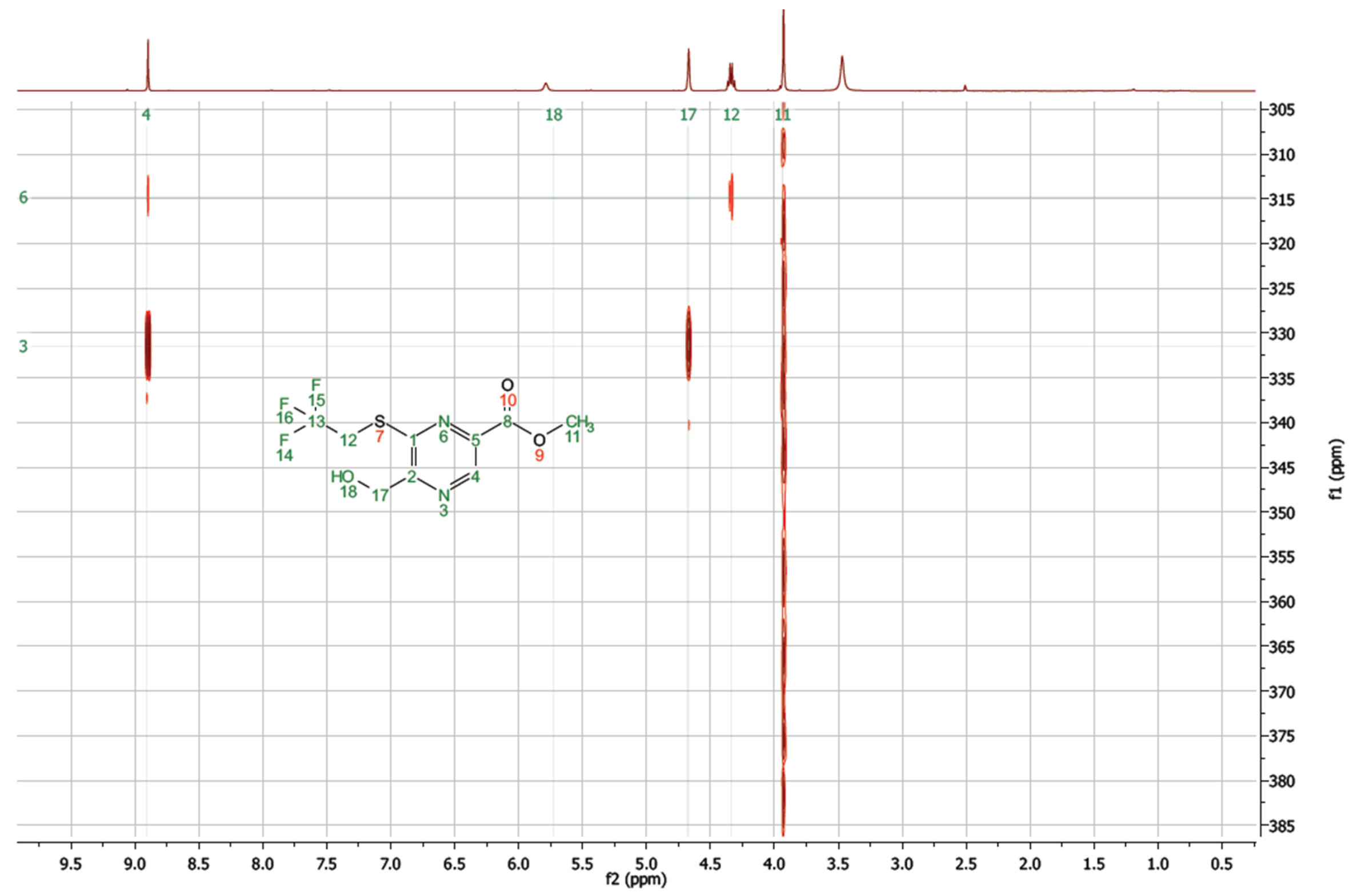


${ }^{1} \mathrm{H}$ NMR spectrum of $\mathbf{3 b}\left(500 \mathrm{MHz}, \mathrm{CD}_{3} \mathrm{OD}\right)$

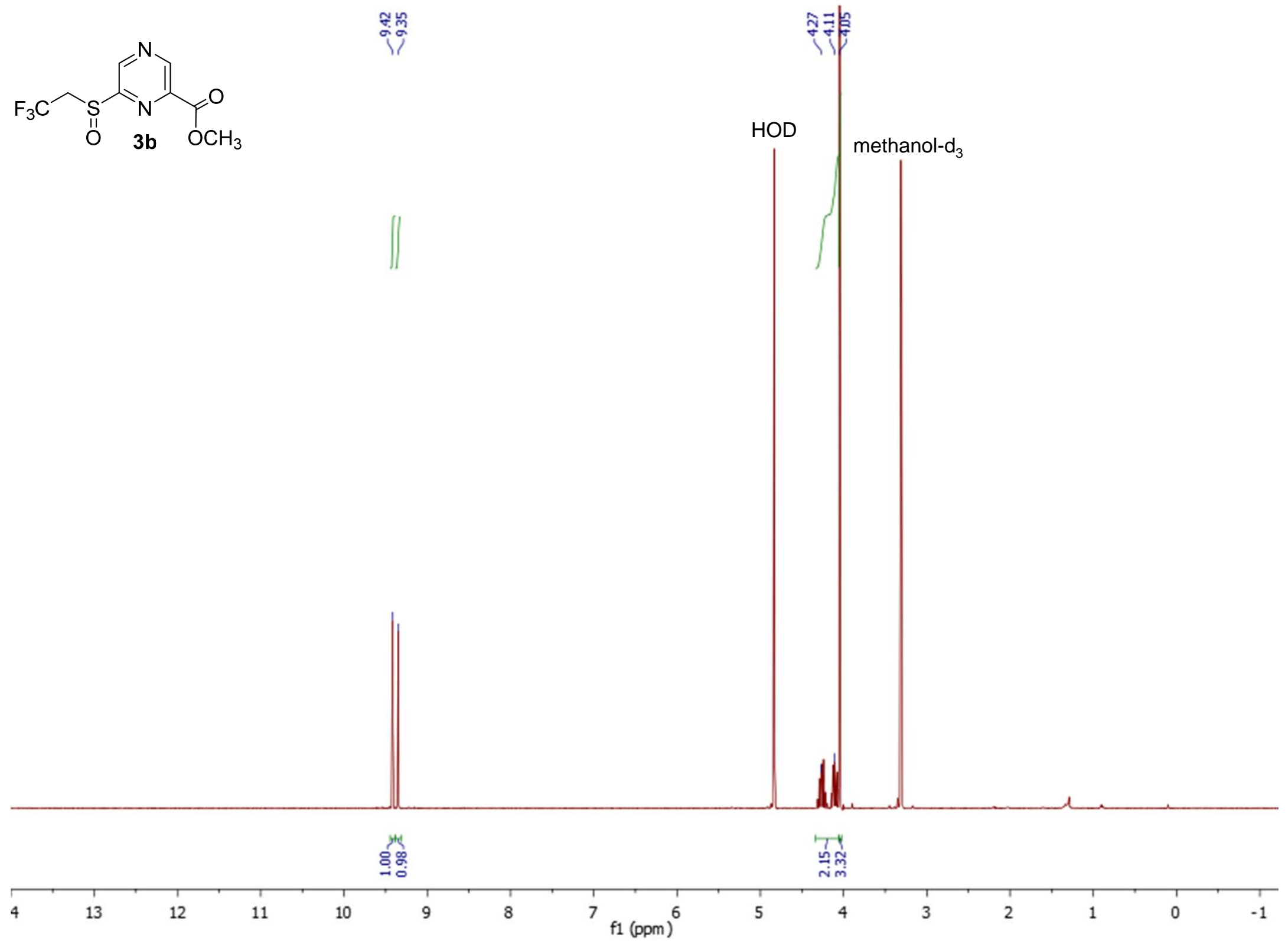


${ }^{13} \mathrm{C}$ NMR spectrum of $\mathbf{3 b}\left(125 \mathrm{MHz}, \mathrm{CD}_{3} \mathrm{OD}\right)$
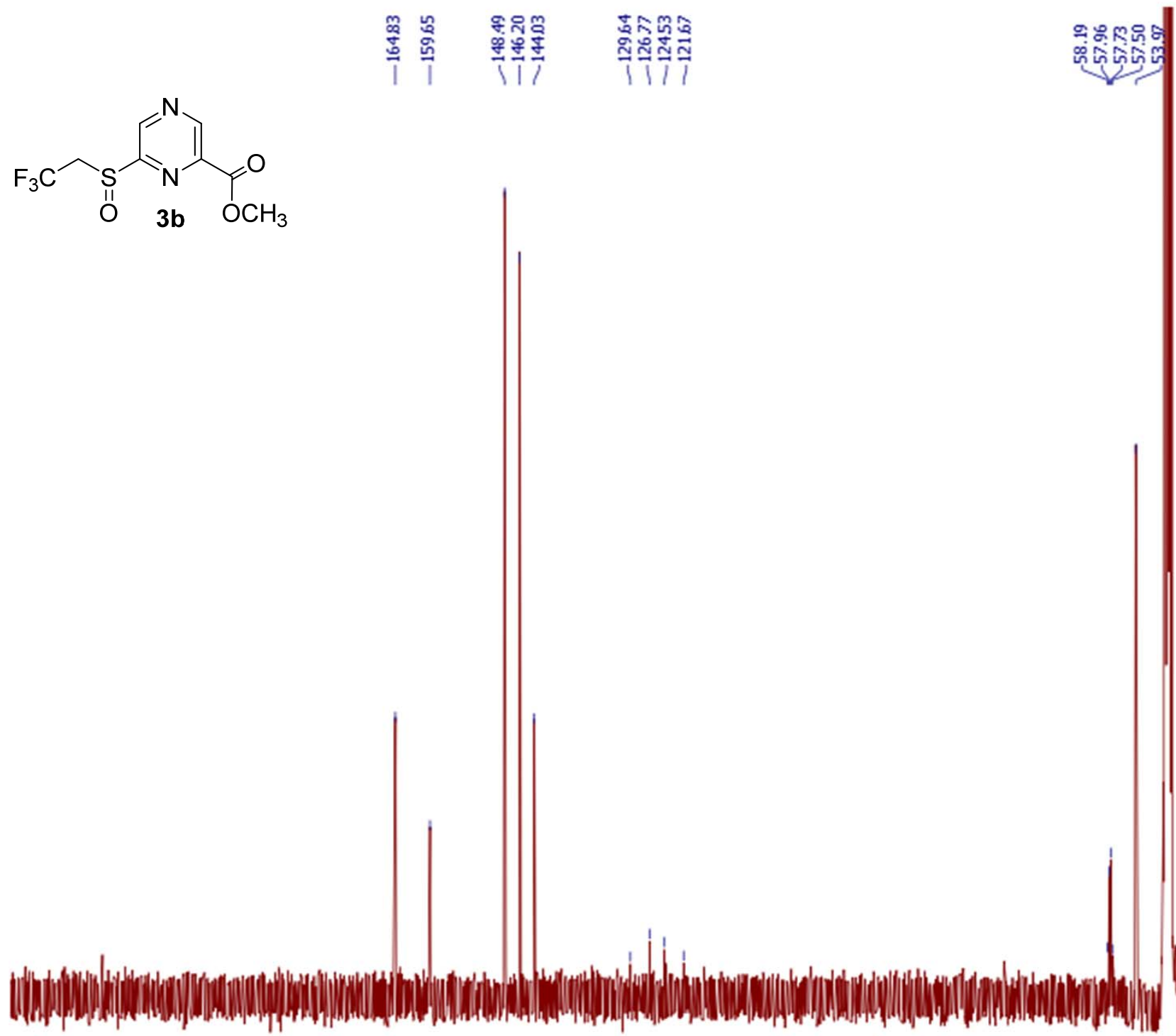

methanol- $\mathrm{d}_{4}$

$220 \quad 210 \quad 200$

190

170

160

140

130

$\underset{\mathrm{f} 1(\mathrm{ppm})}{110}$

90

60

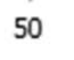


${ }^{19} \mathrm{~F}$ NMR spectrum of $\mathbf{3 b}\left(470 \mathrm{MHz}, \mathrm{CD}_{3} \mathrm{OD}\right)$
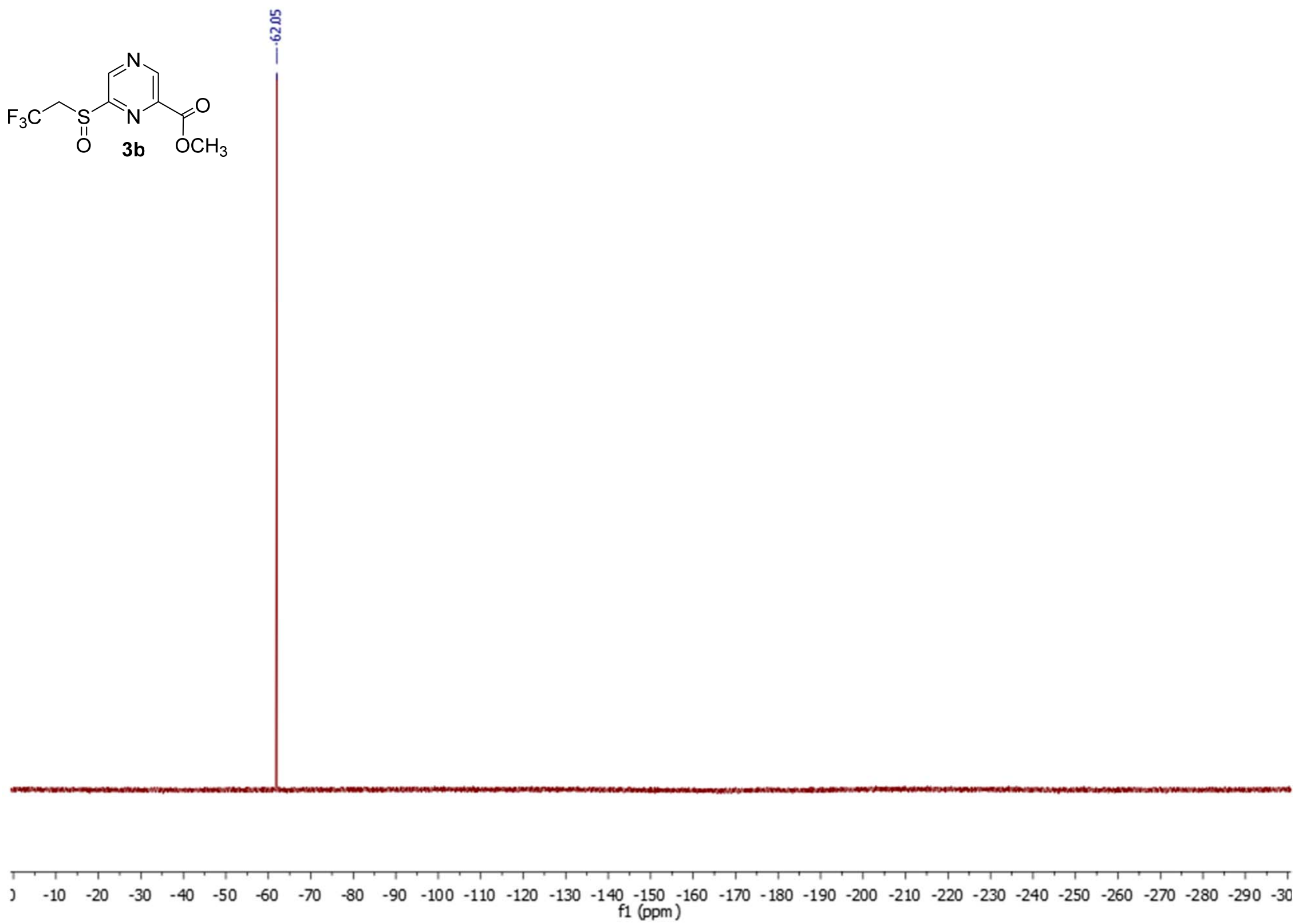
${ }^{1} \mathrm{H}$ NMR spectrum of $\mathbf{4 a}\left(600 \mathrm{MHz}, \mathrm{DMSO}-\mathrm{d}_{6}\right)$

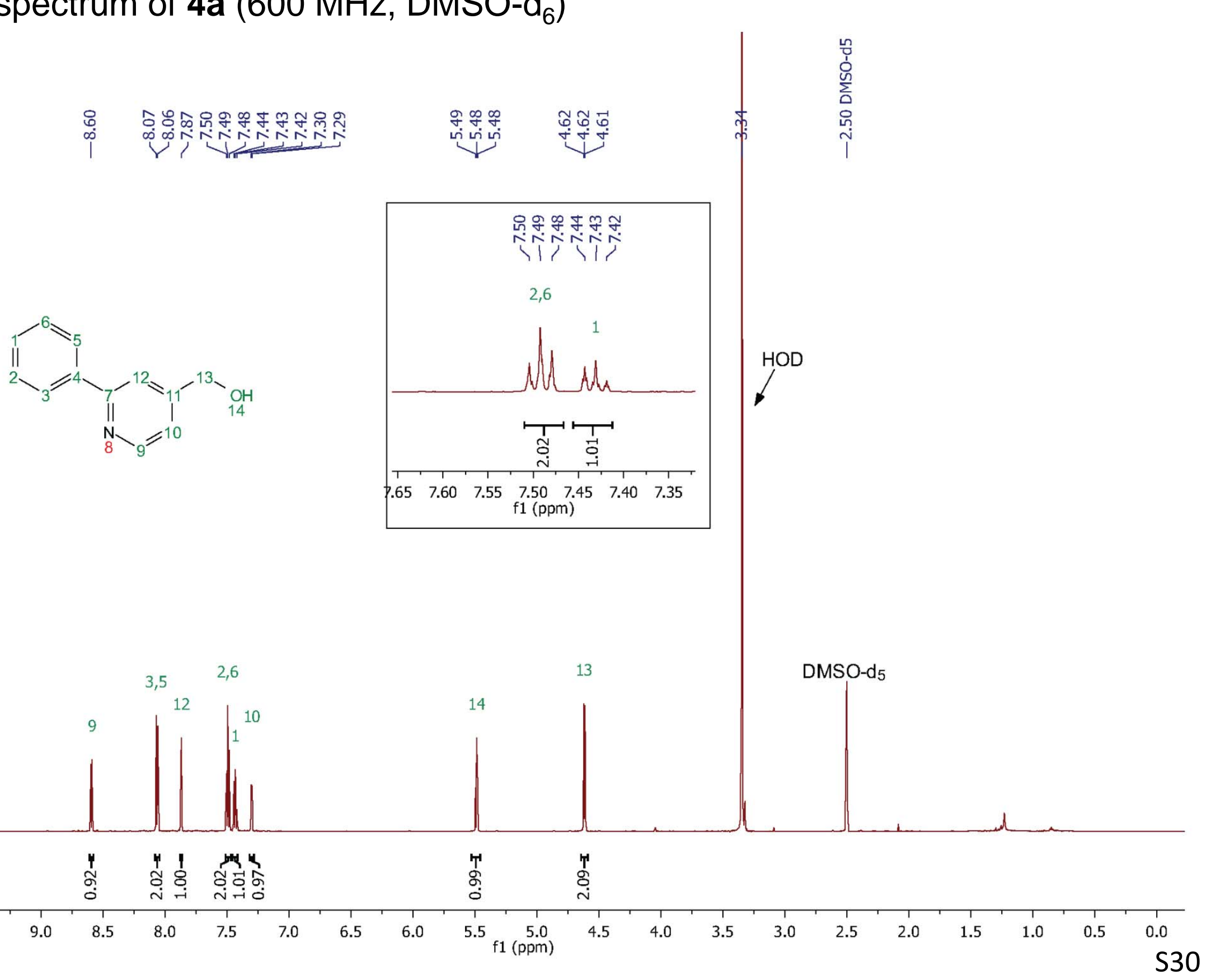


${ }^{13} \mathrm{C}$ NMR spectrum of $\mathbf{4 a}\left(150 \mathrm{MHz}, \mathrm{DMSO}-\mathrm{d}_{6}\right)$
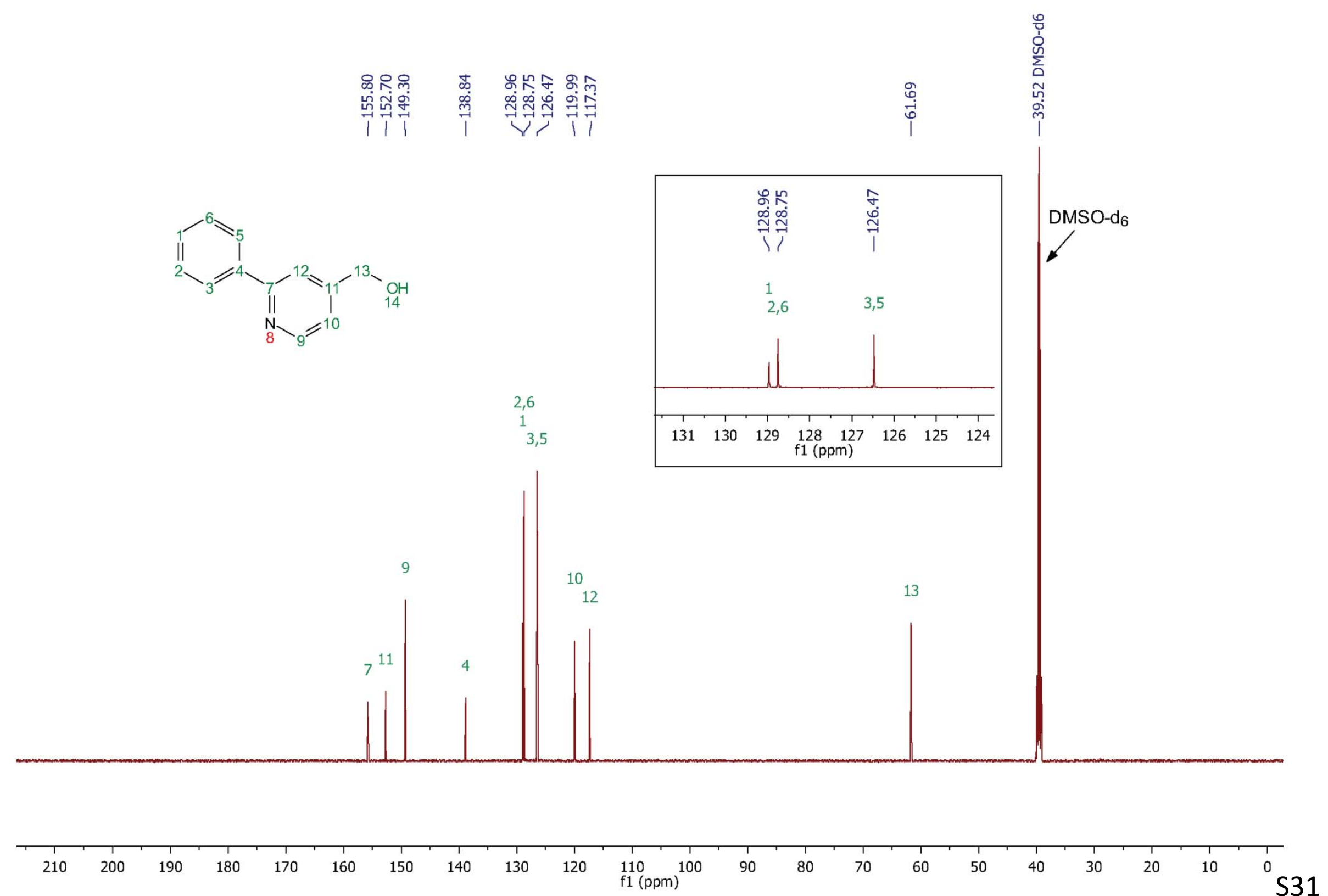
${ }^{1} \mathrm{H} /{ }^{1} \mathrm{H}$ COSY NMR spectrum of $4 \mathrm{a}\left(600 / 600 \mathrm{MHz}\right.$, DMSO- $\left.\mathrm{d}_{6}\right)$

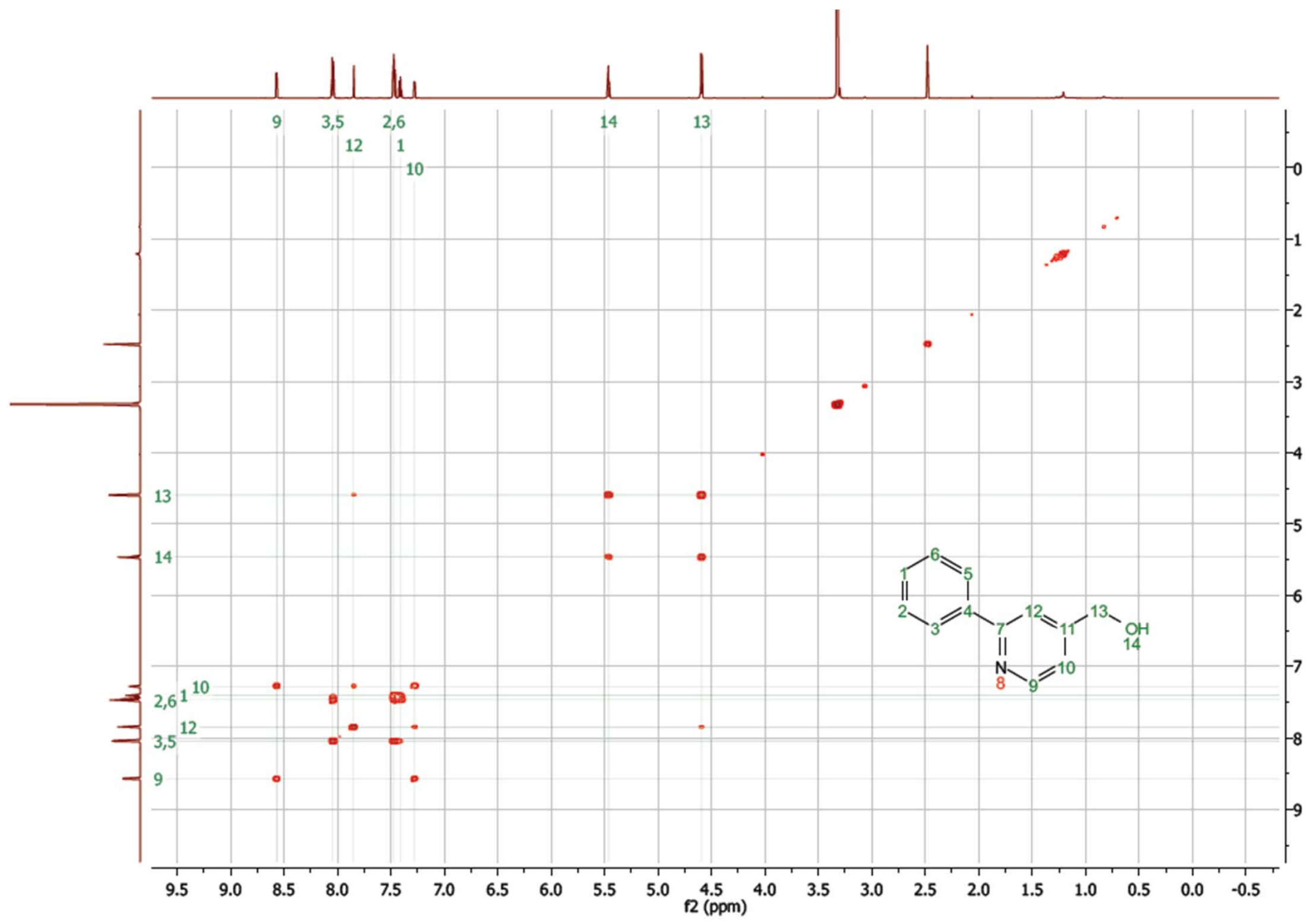


${ }^{1} \mathrm{H} /{ }^{13} \mathrm{C}$ HMBC NMR spectrum of $4 \mathrm{a}\left(600 / 150 \mathrm{MHz}\right.$, DMSO-d $\left.\mathrm{d}_{6}\right)$

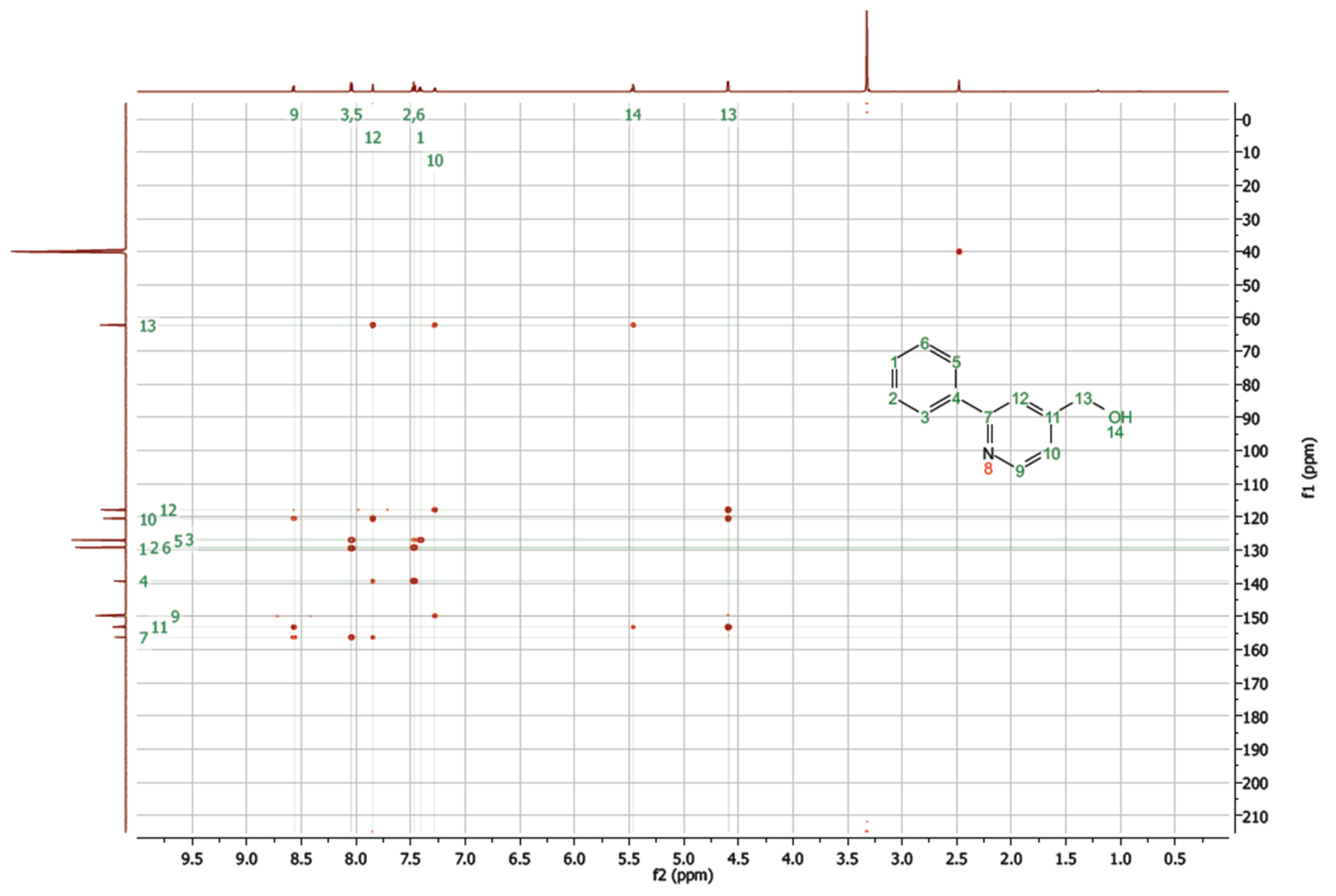


${ }^{1} \mathrm{H} /{ }^{13} \mathrm{C}$ HSQC NMR spectrum of $4 a\left(600 / 150 \mathrm{MHz}\right.$, DMSO-d $\left.\mathrm{d}_{6}\right)$

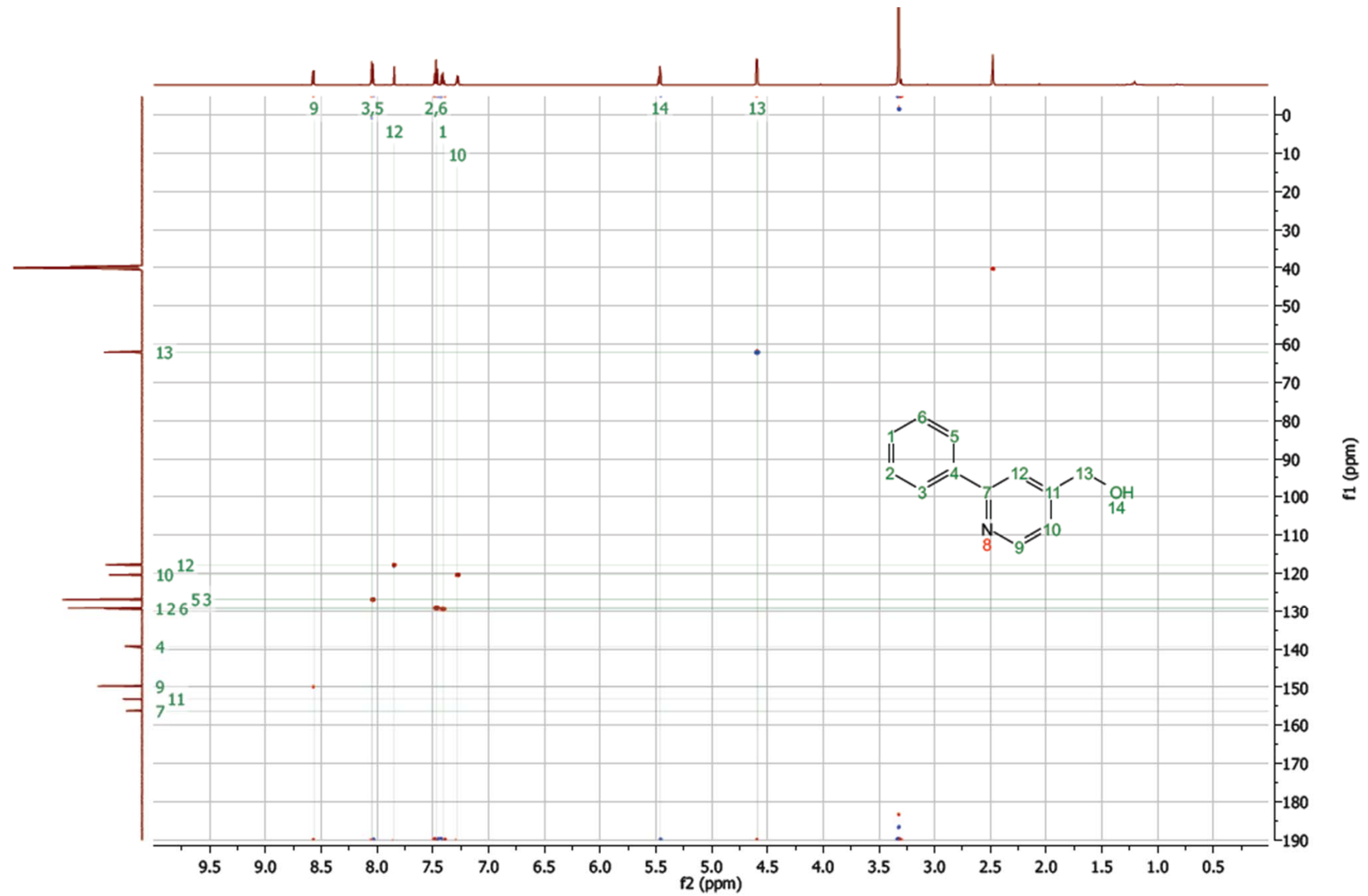


${ }^{1} \mathrm{H} /{ }^{1} \mathrm{H}$ ROESY NMR spectrum of $4 \mathbf{a}\left(600 / 600 \mathrm{MHz}\right.$, DMSO-d $\left.\mathrm{d}_{6}\right)$

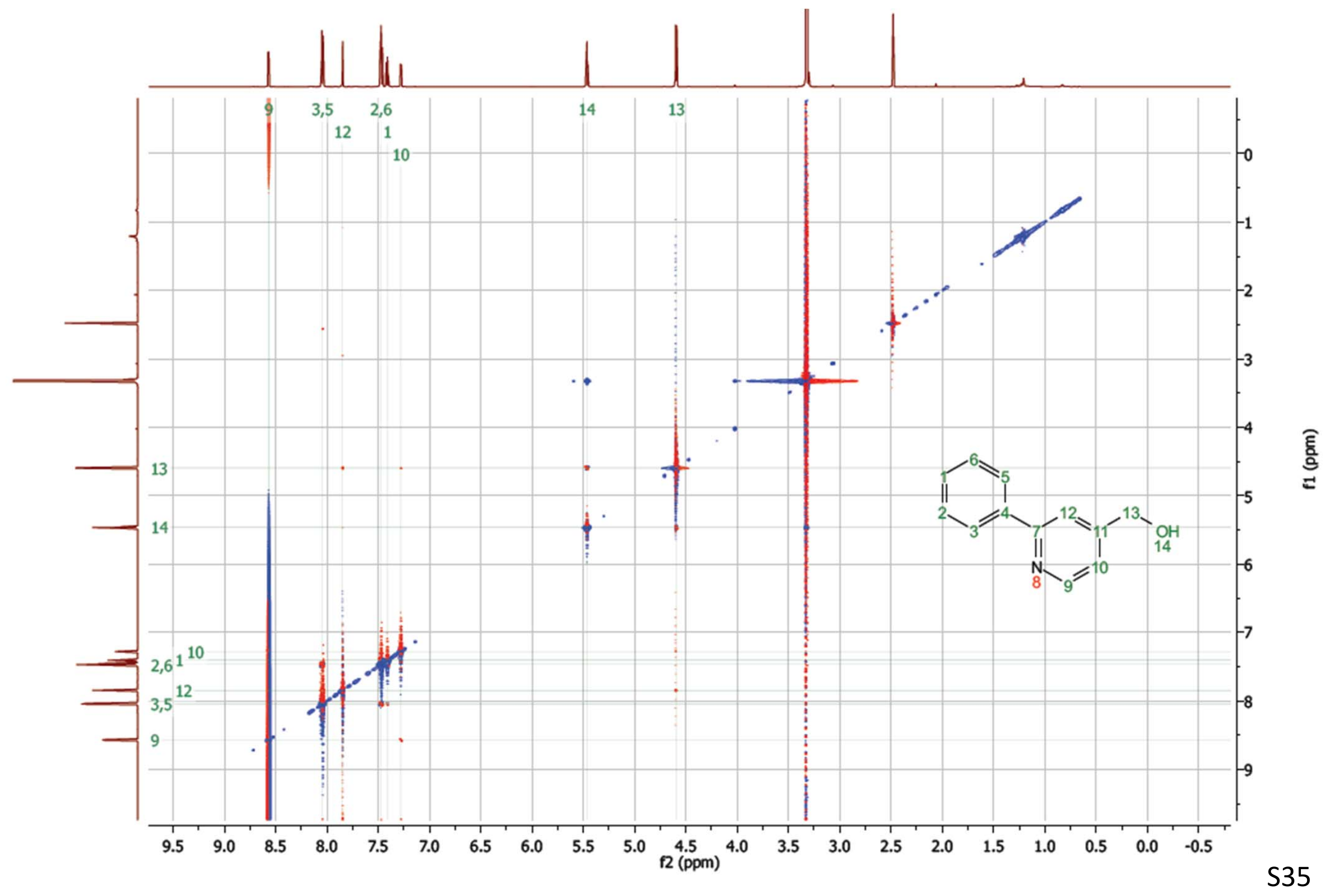


${ }^{1} \mathrm{H}$ NMR spectrum of $\mathbf{4 b}\left(500 \mathrm{MHz}\right.$, DMSO- $\left.\mathrm{d}_{6}\right)$

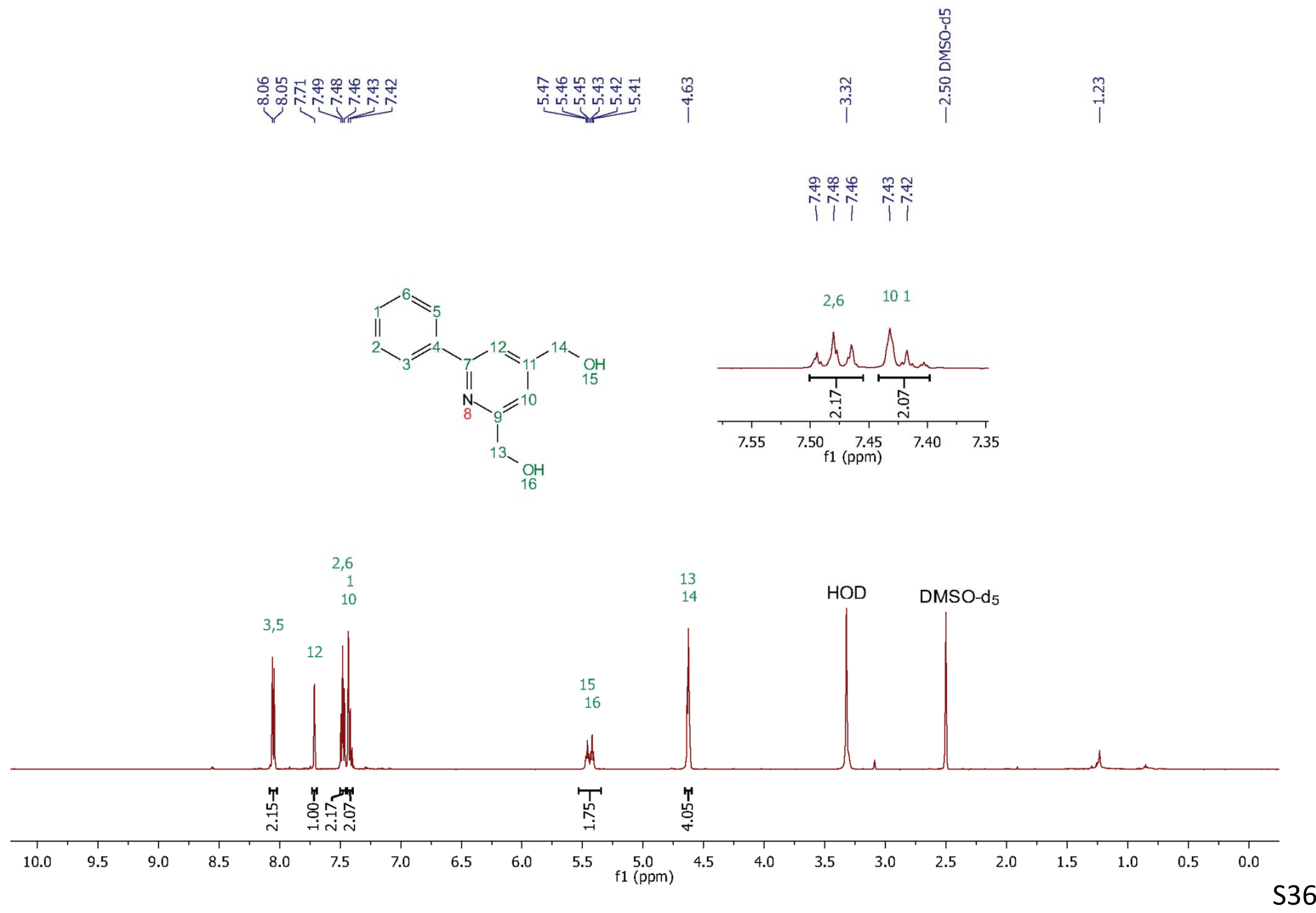


${ }^{13} \mathrm{C}$ NMR spectrum of $\mathbf{4 b}\left(125 \mathrm{MHz}, \mathrm{DMSO}-\mathrm{d}_{6}\right)$
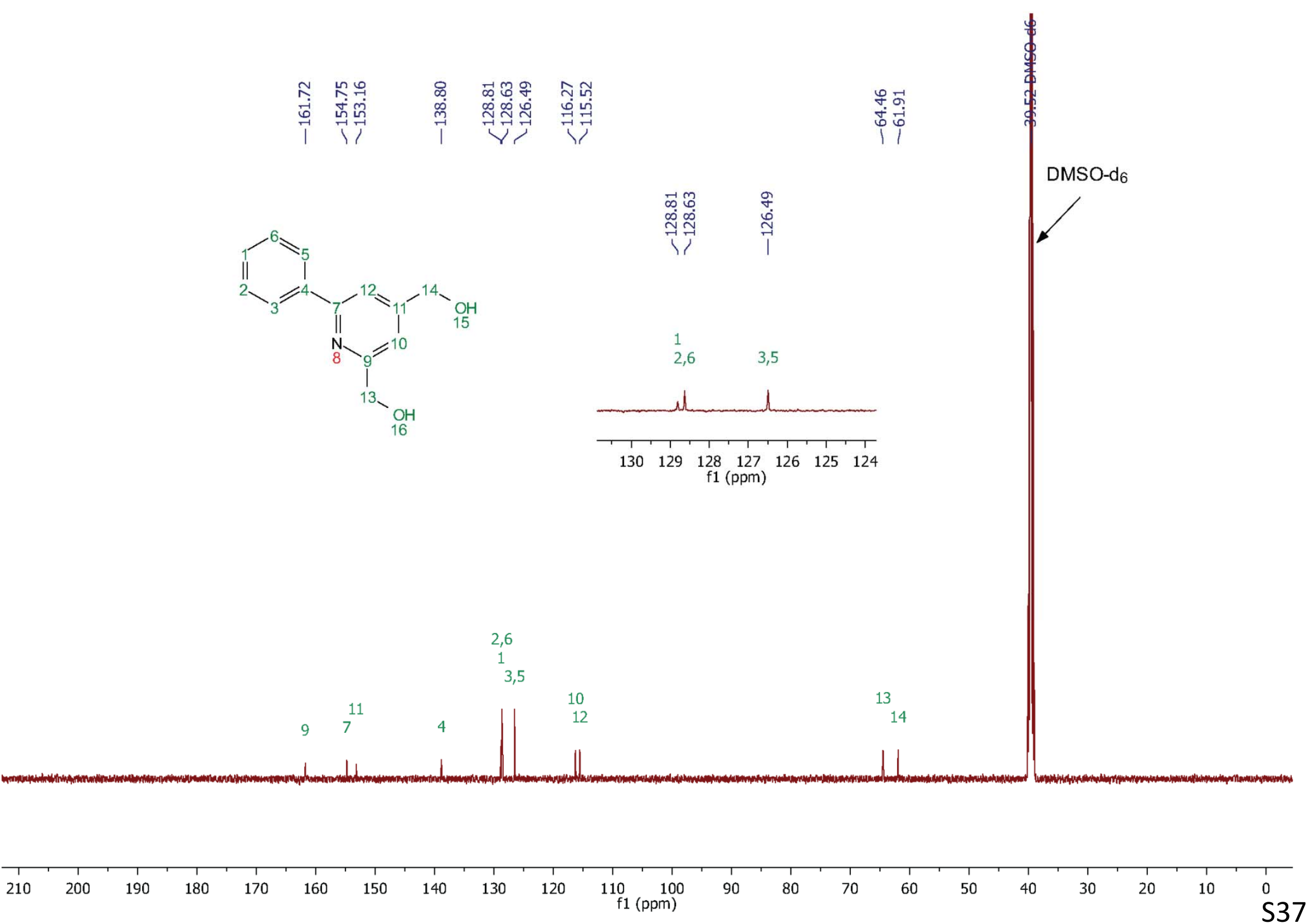
${ }^{1} \mathrm{H} /{ }^{1} \mathrm{H}$ COSY NMR spectrum of $4 \mathbf{b}\left(500 / 500 \mathrm{MHz}\right.$, DMSO- $\left.\mathrm{d}_{6}\right)$

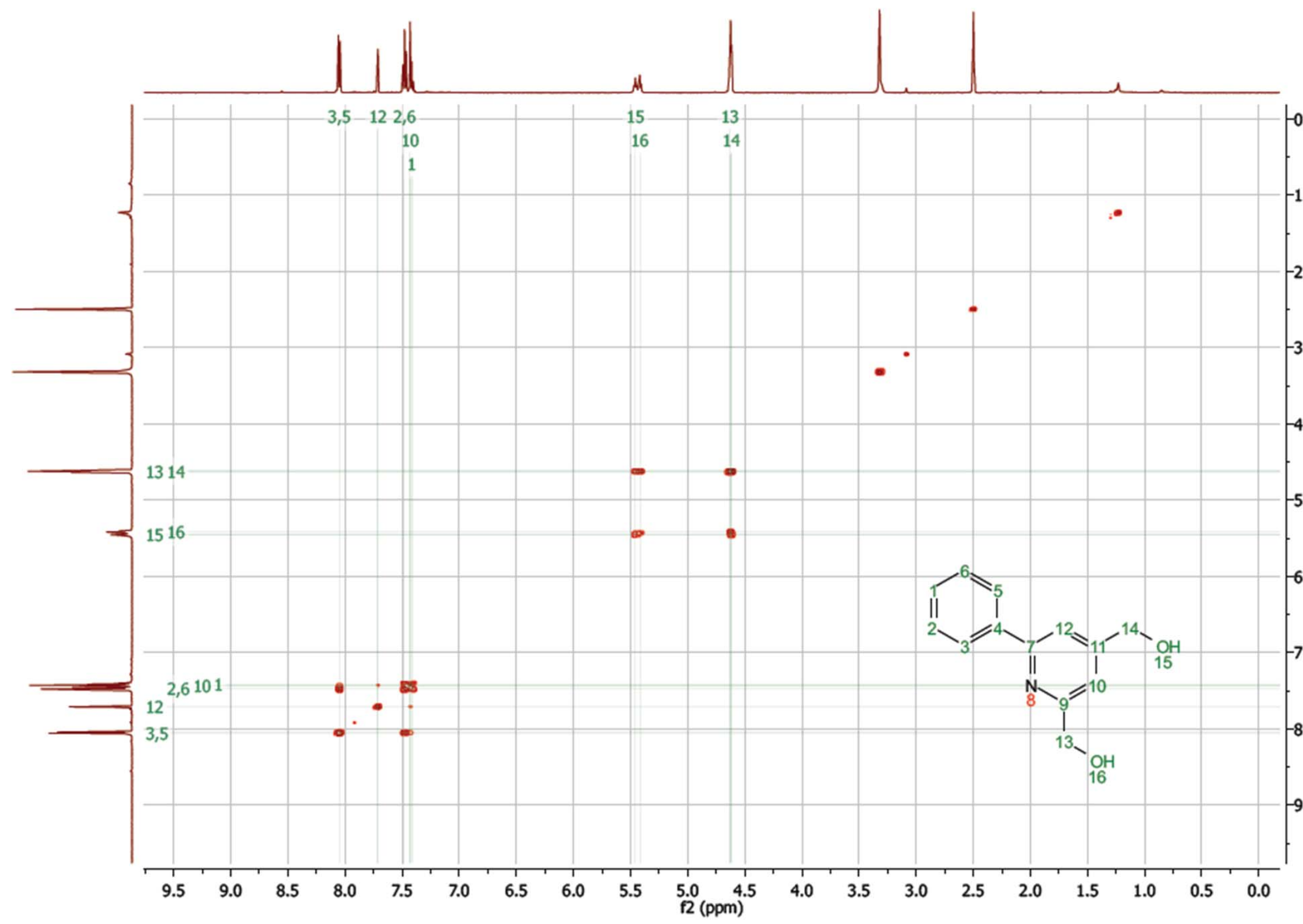

흥 
${ }^{1} \mathrm{H} /{ }^{13} \mathrm{C}$ HSQC NMR spectrum of $4 \mathbf{b}\left(500 / 125 \mathrm{MHz}\right.$, DMSO- $\left.\mathrm{d}_{6}\right)$

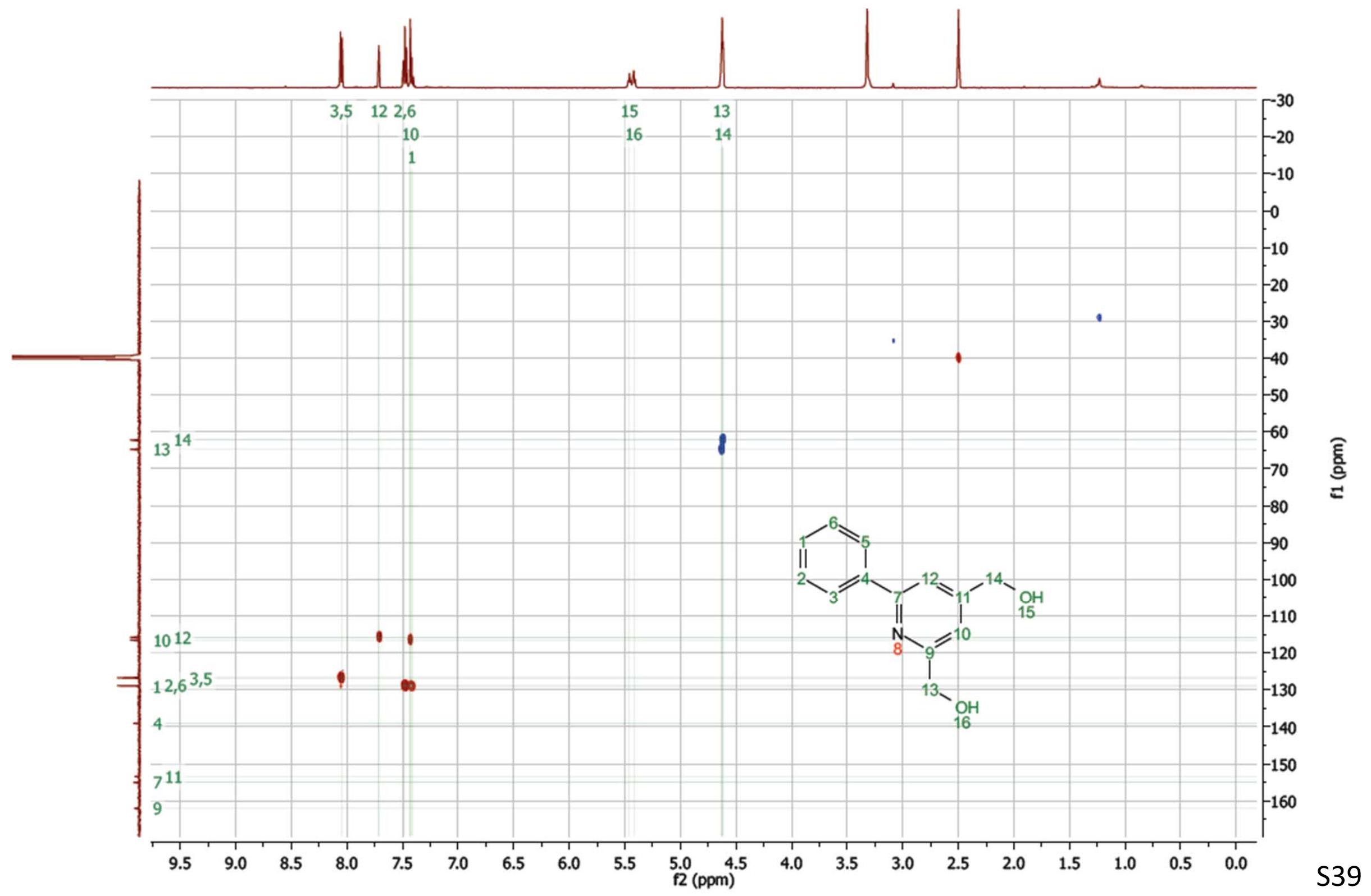


${ }^{1} \mathrm{H} /{ }^{13} \mathrm{C}$ HMBC NMR spectrum of $4 \mathbf{b}\left(500 / 125 \mathrm{MHz}\right.$, DMSO-d $\left.\mathrm{d}_{6}\right)$

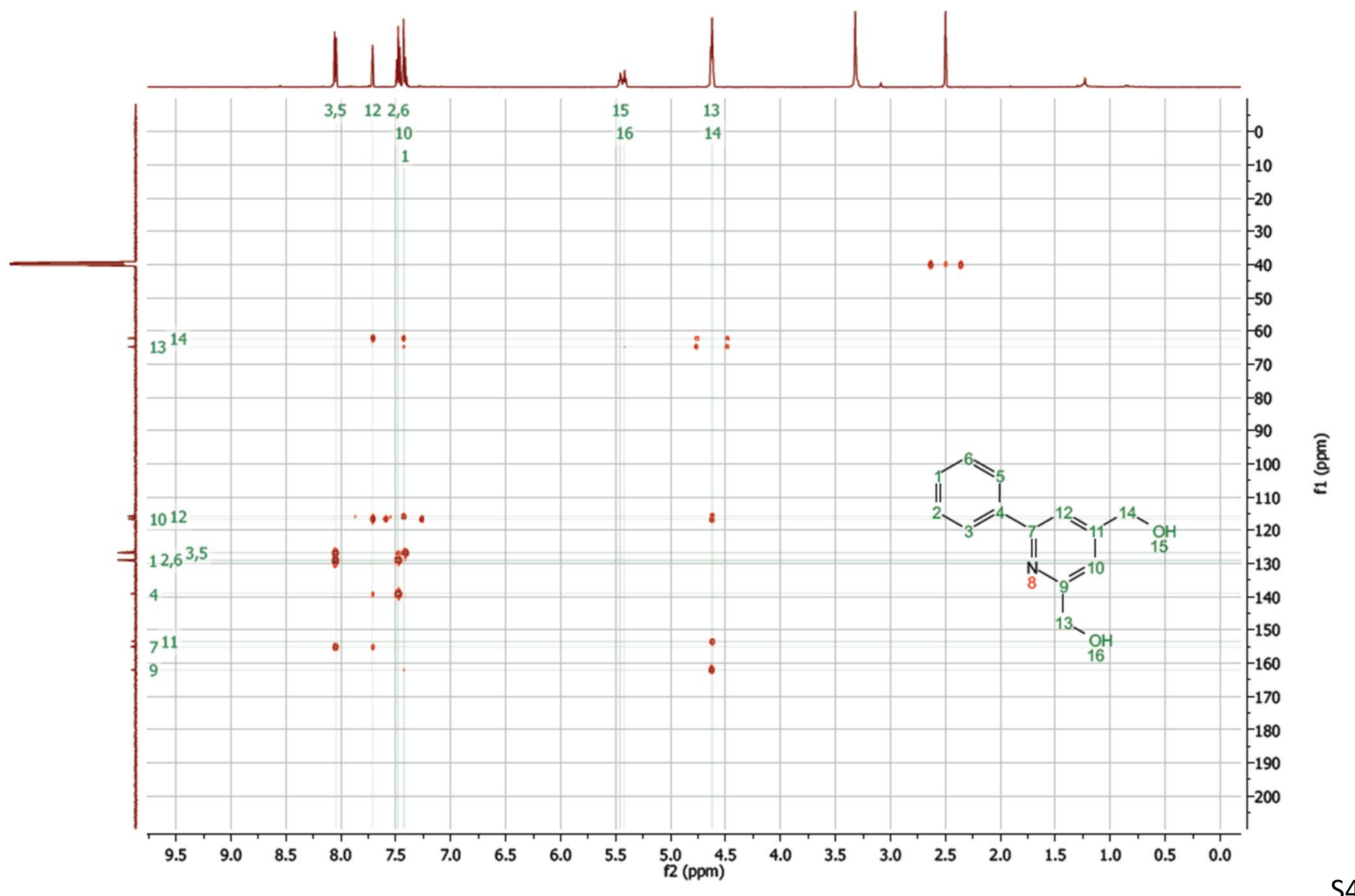


${ }^{1} \mathrm{H} /{ }^{1} \mathrm{H}$ ROESY of $\mathbf{4 b}\left(500 / 500 \mathrm{MHz}\right.$, DMSO- $\left.\mathrm{d}_{6}\right)$

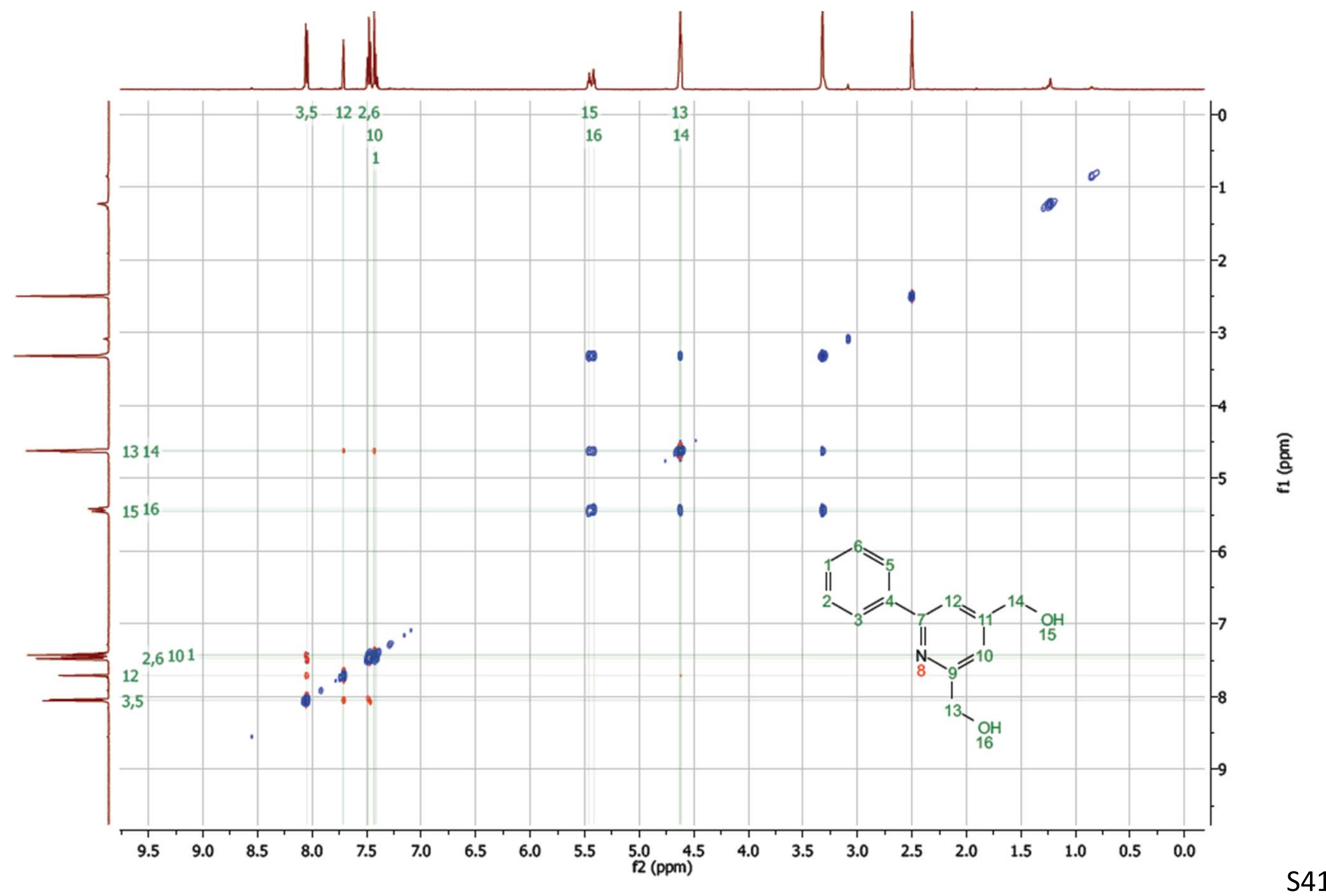


${ }^{1} \mathrm{H}$ NMR spectrum of 5a.TFA (500 MHz, DMSO- $\mathrm{d}_{6}$ )

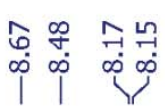
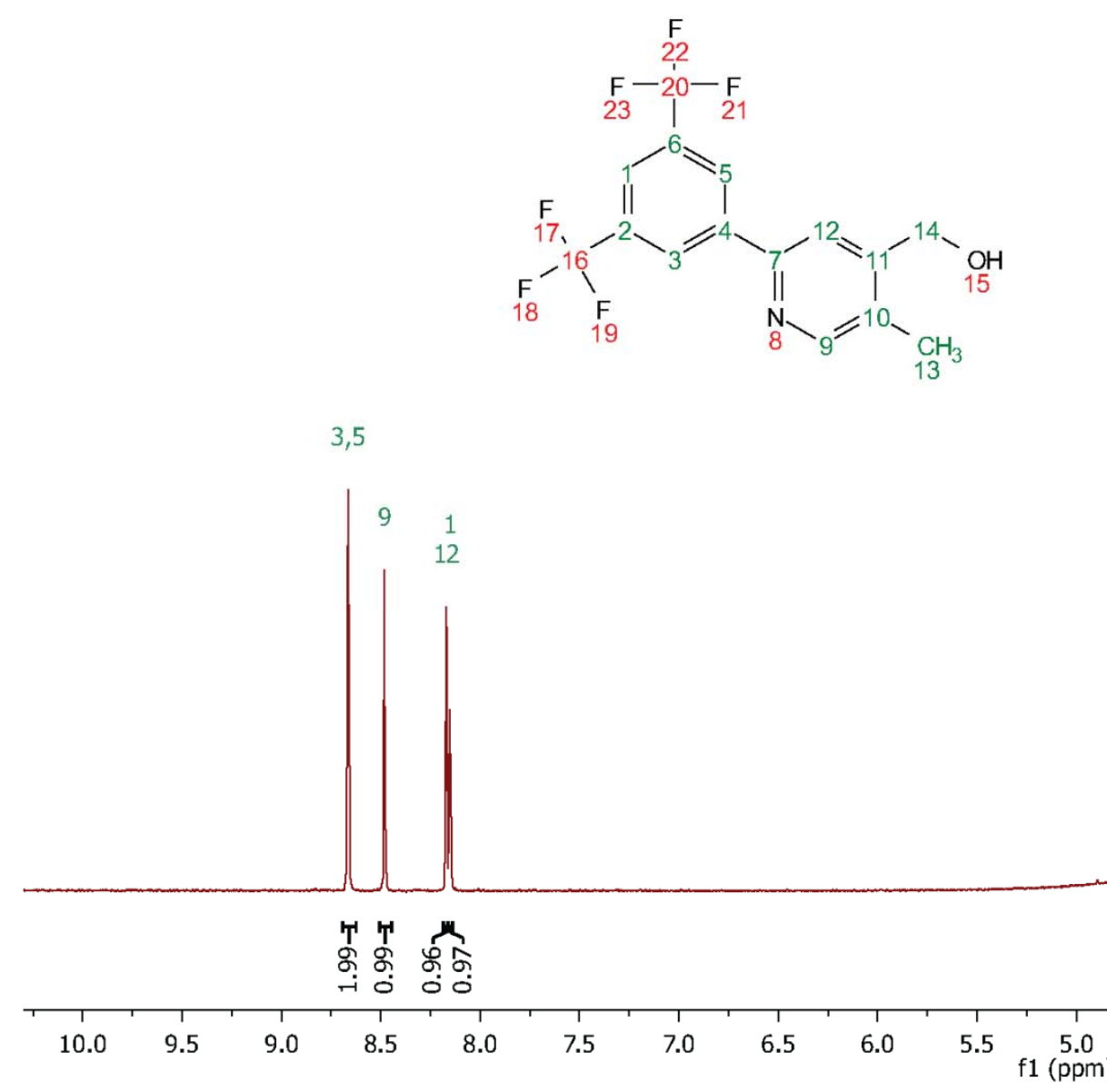

14

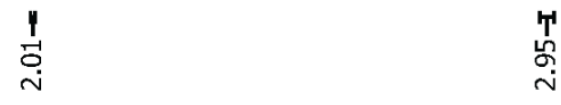

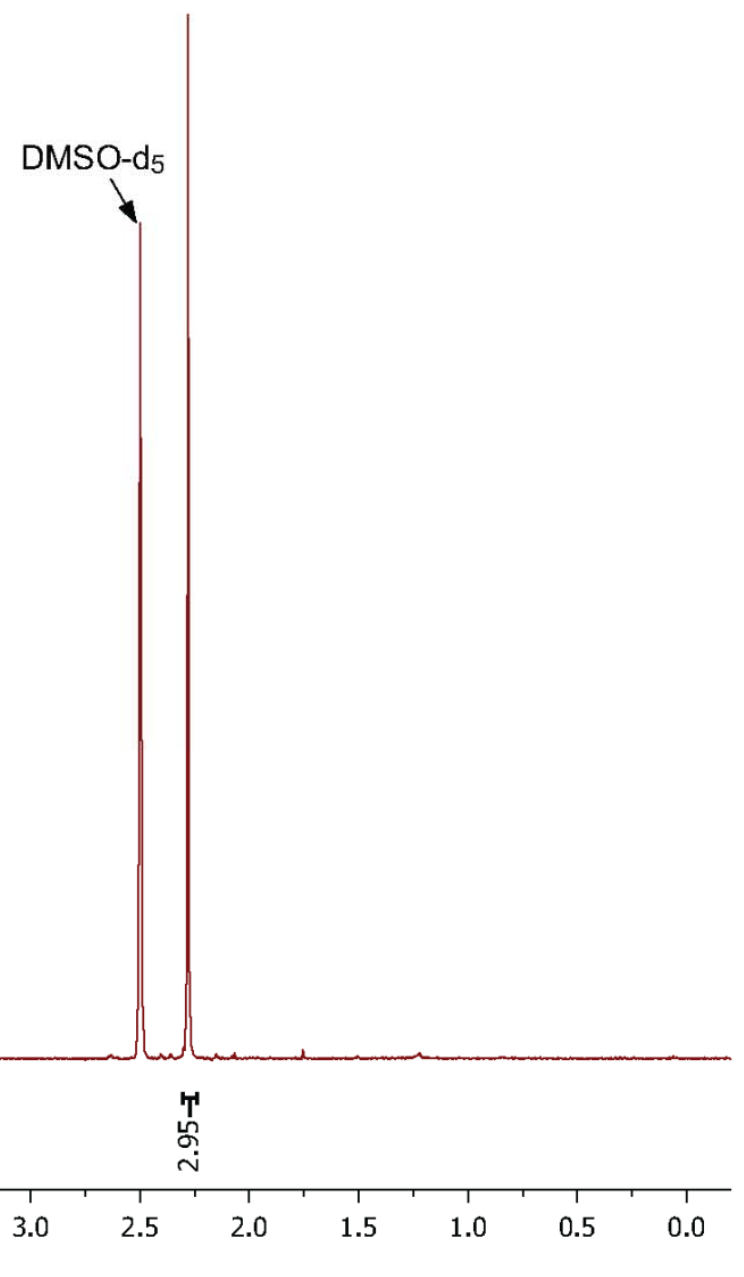


${ }^{13} \mathrm{C}$ NMR spectrum of 5a•TFA (125 MHz, DMSO-d $\left.{ }_{6}\right)$

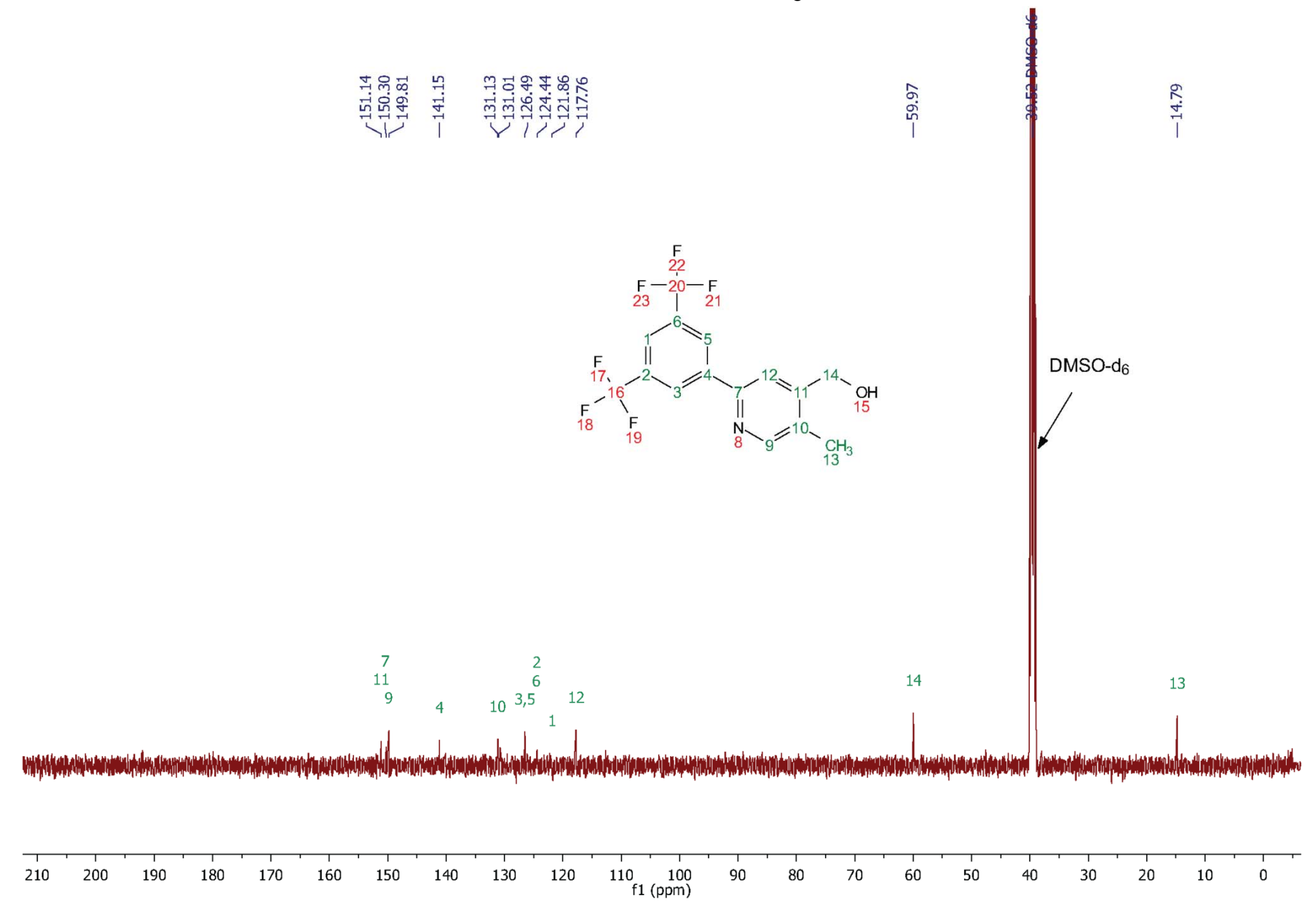


${ }^{19} \mathrm{~F}$ NMR spectrum of 5a・TFA (470 MHz, DMSO-d $\left.{ }_{6}\right)$

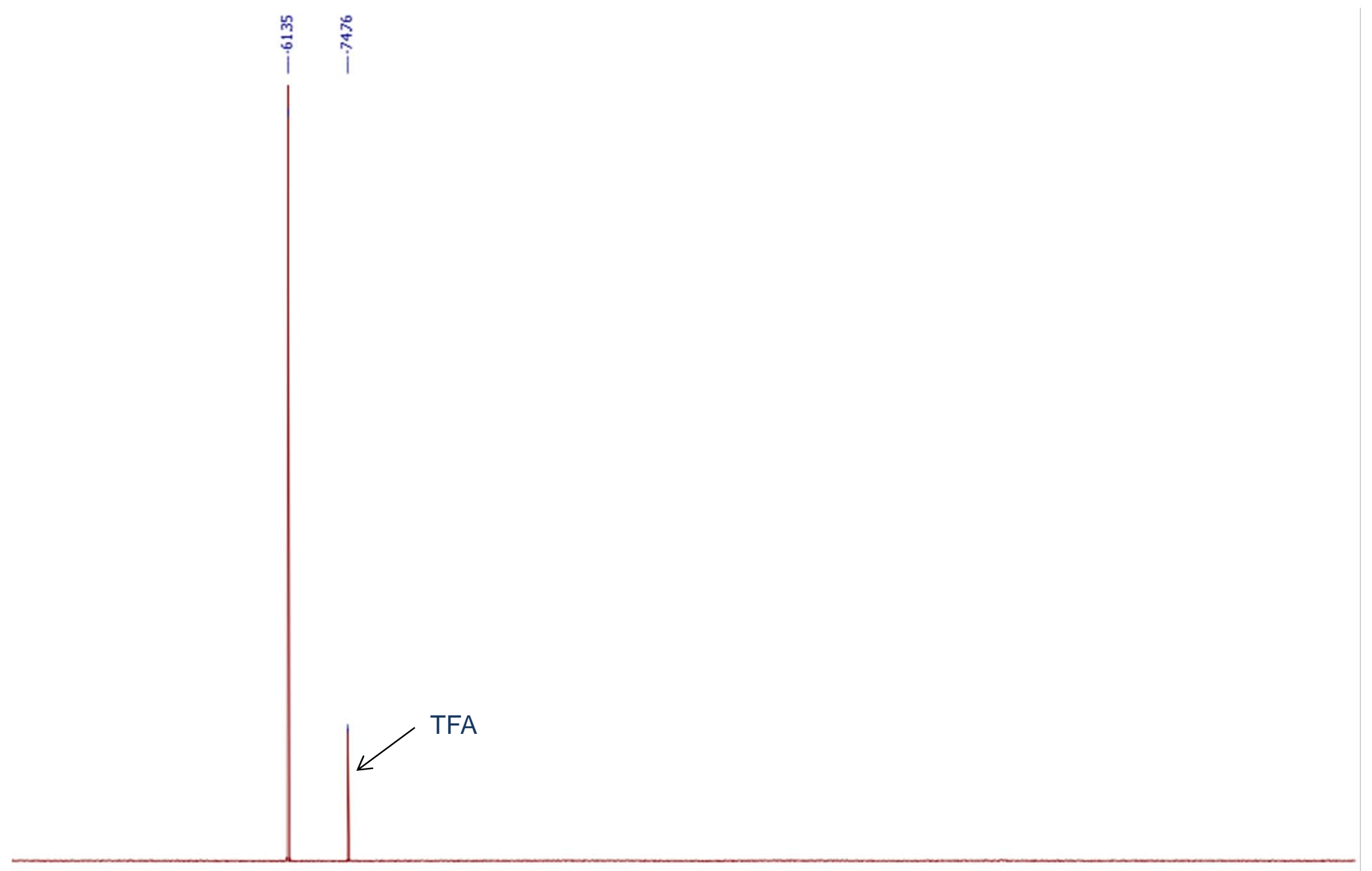


${ }^{1} \mathrm{H} /{ }^{1} \mathrm{H}$ COSY NMR spectrum of 5a・TFA $\left(500 / 500 \mathrm{MHz}\right.$, DMSO- $\left.\mathrm{d}_{6}\right)$

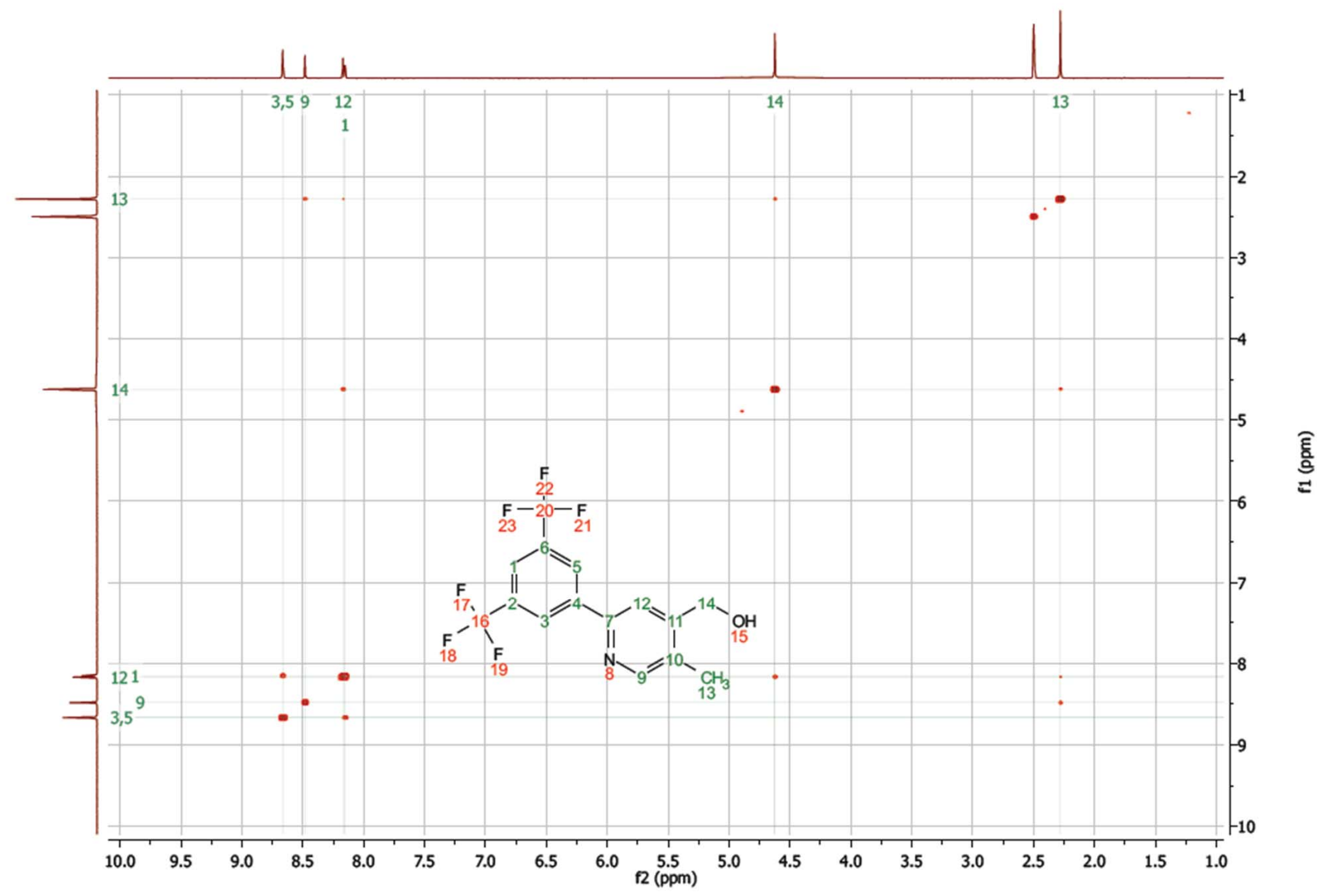


${ }^{1} \mathrm{H} /{ }^{13} \mathrm{C}$ HSQC NMR spectrum of 5a•TFA (500 / $125 \mathrm{MHz}$, DMSO-d ) $^{2}$

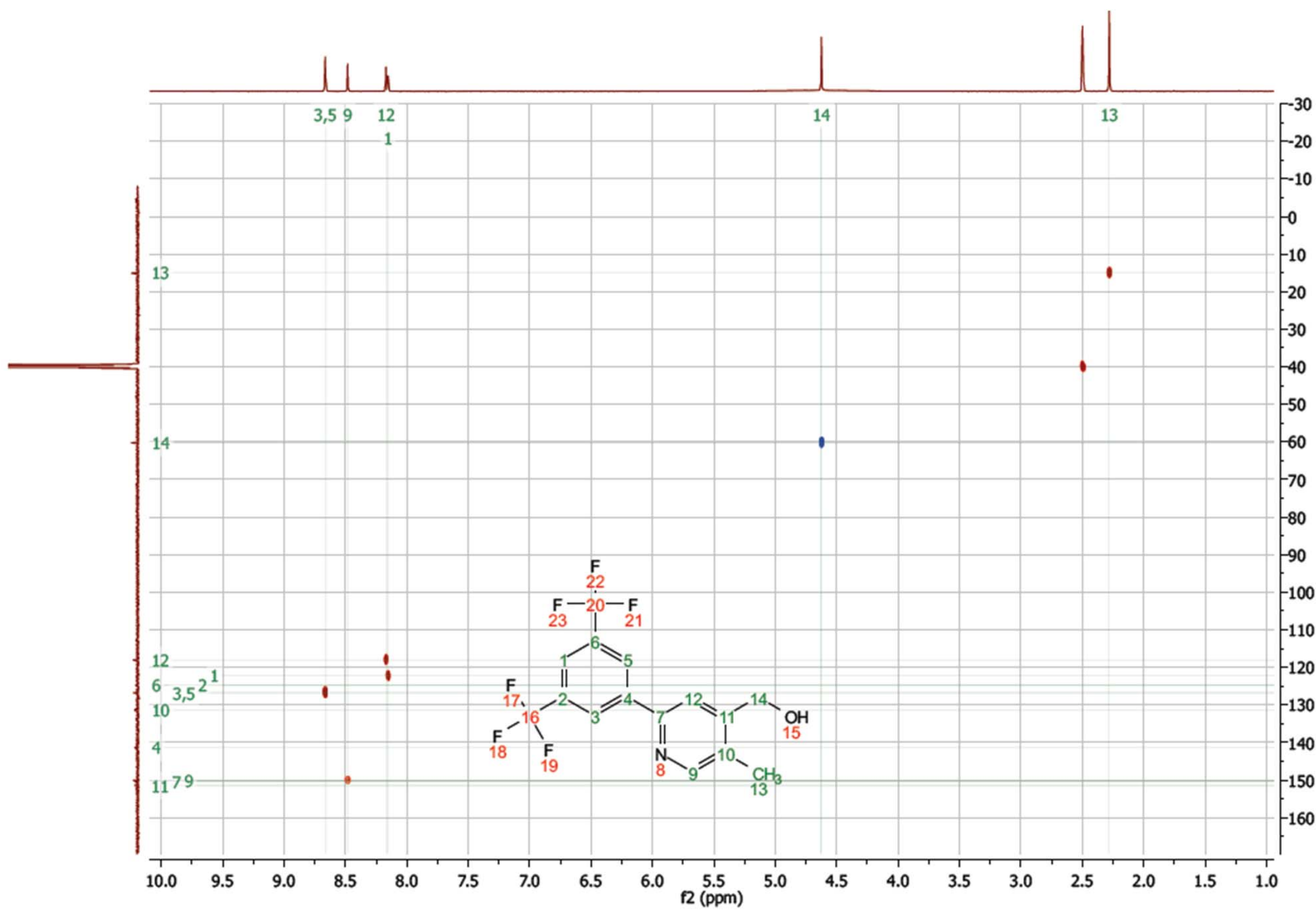


${ }^{1} \mathrm{H} /{ }^{13} \mathrm{C}$ HMBC NMR spectrum of 5a•TFA (500 / $125 \mathrm{MHz}$, DMSO- $\mathrm{d}_{6}$ )

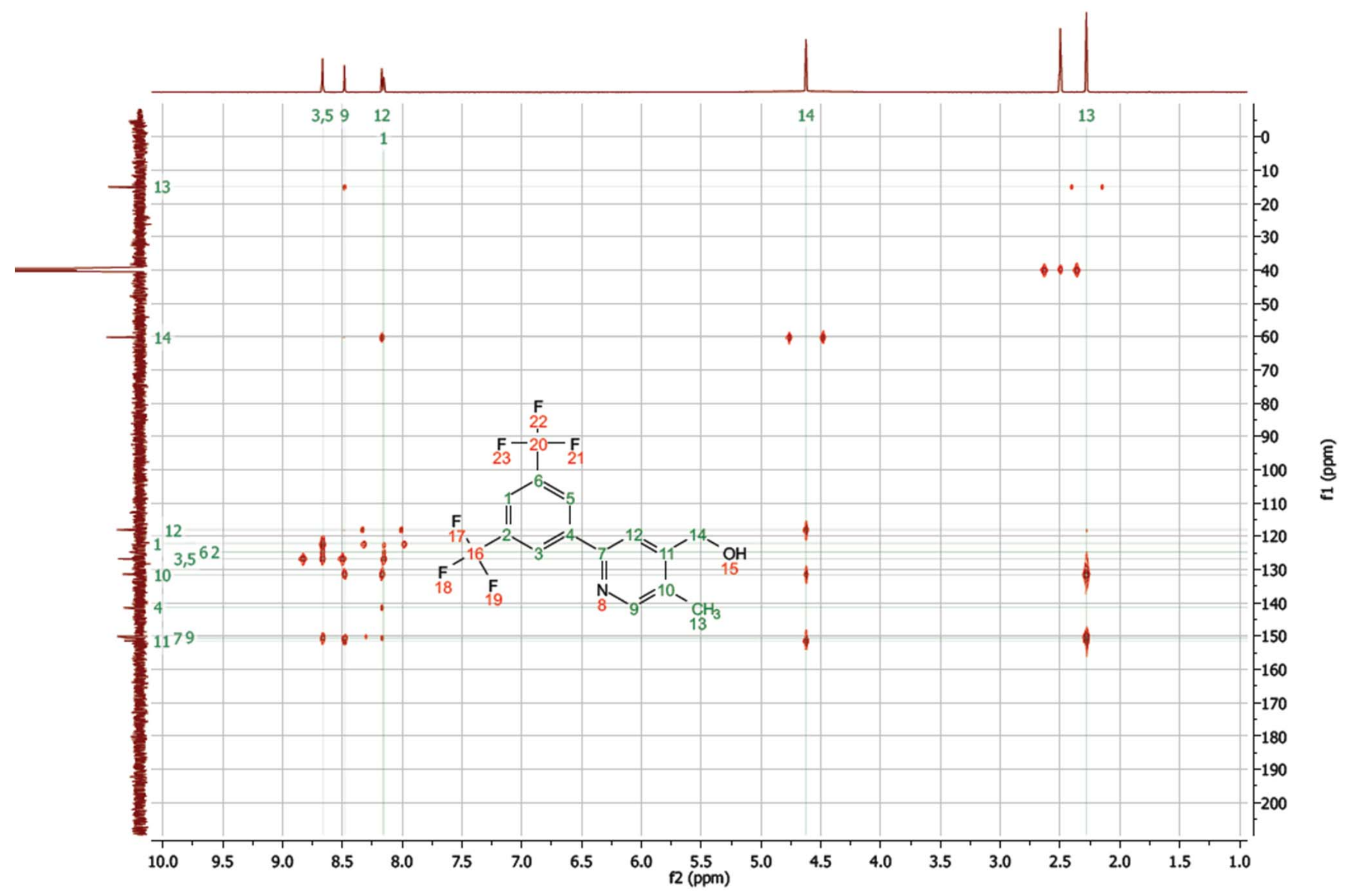


${ }^{1} \mathrm{H} /{ }^{1} \mathrm{H}$ ROESY NMR spectrum of 5a•TFA (500 / $500 \mathrm{MHz}$, DMSO-d $\mathrm{d}_{6}$ )

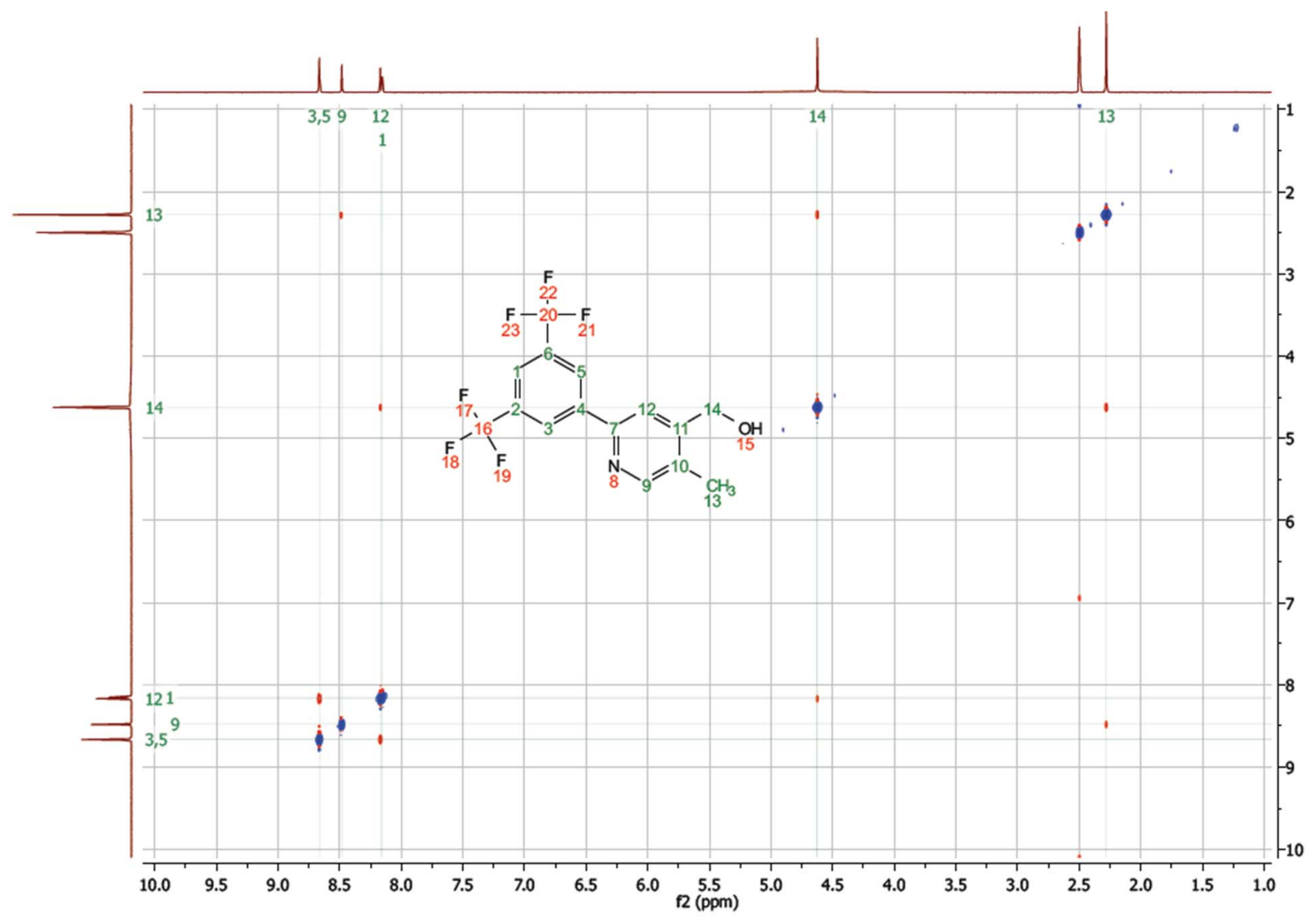


${ }^{1} \mathrm{H}$ NMR spectrum of 5b•TFA (600 MHz, DMSO- $\left.\mathrm{d}_{6}\right)$

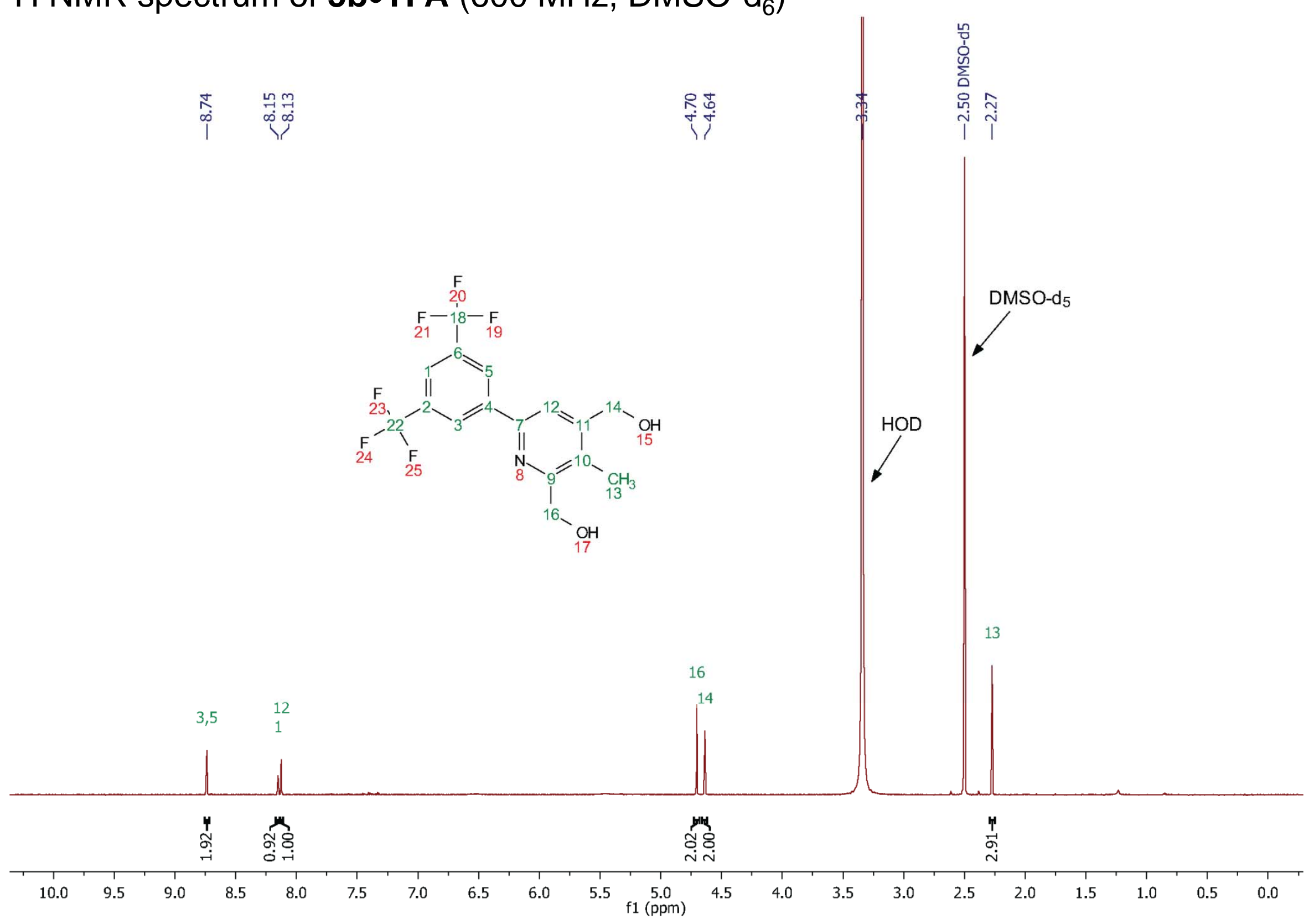


${ }^{13} \mathrm{C}$ NMR spectrum of 5b•TFA (150 MHz, DMSO-d $\left.{ }_{6}\right)$

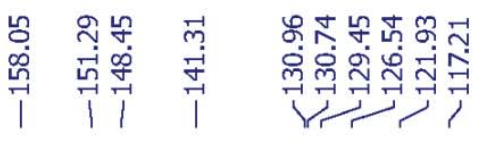
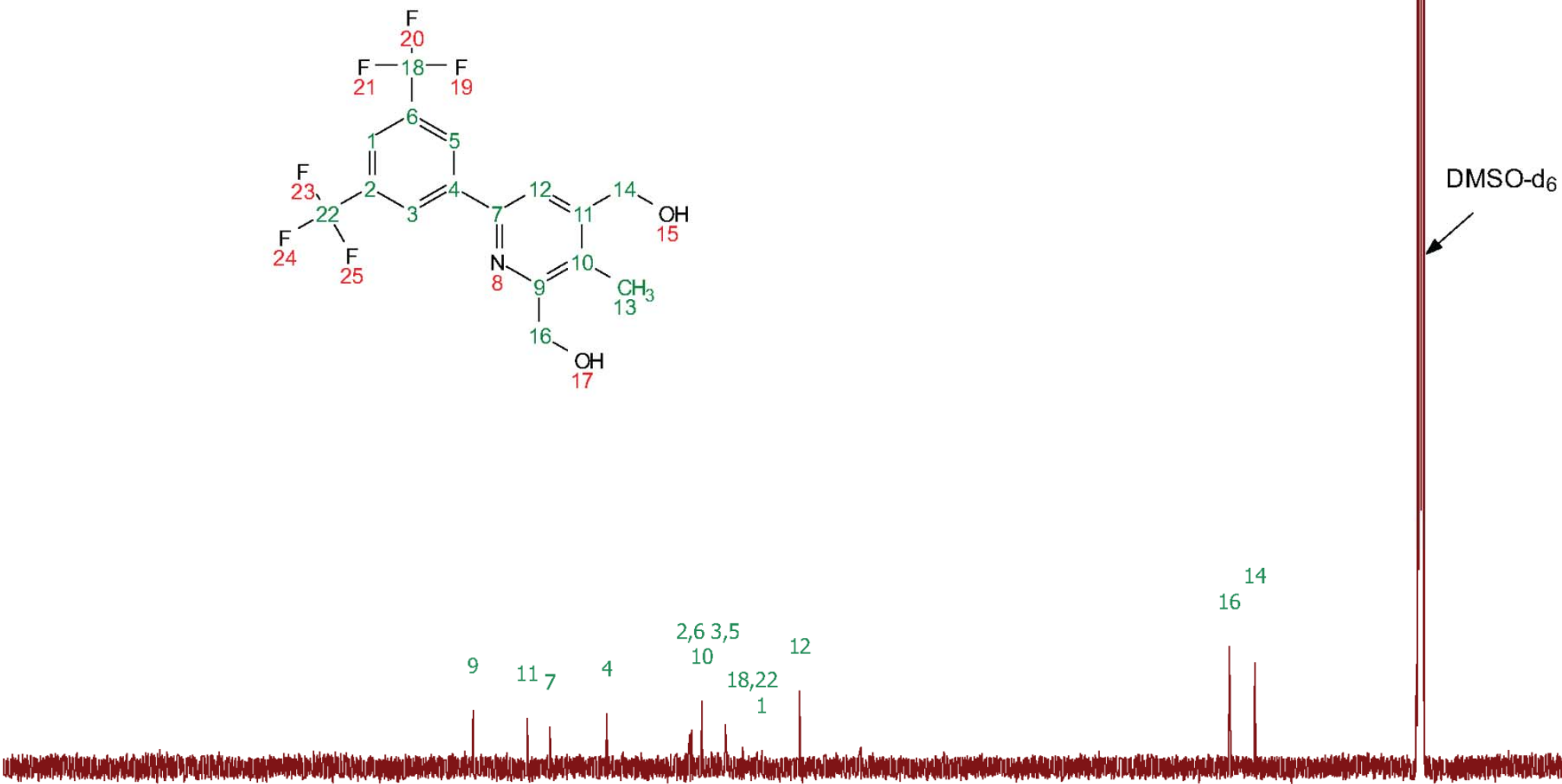

\begin{tabular}{|c|c|c|c|c|c|c|c|c|c|c|c|c|c|c|c|c|c|c|c|c|c|c|}
\hline 210 & 200 & 190 & 180 & 170 & 160 & 150 & & 130 & 120 & $\begin{array}{l}110 \\
\mathrm{f} 1(\mathrm{ppm})\end{array}$ & 90 & 80 & 70 & \multicolumn{2}{|c|}{60} & 5 & & 40 & \multicolumn{2}{|c|}{30} & 20 & 10 \\
\hline
\end{tabular}


${ }^{19} \mathrm{~F}$ NMR spectrum of 5b•TFA (470 MHz, DMSO-d $\left.{ }_{6}\right)$
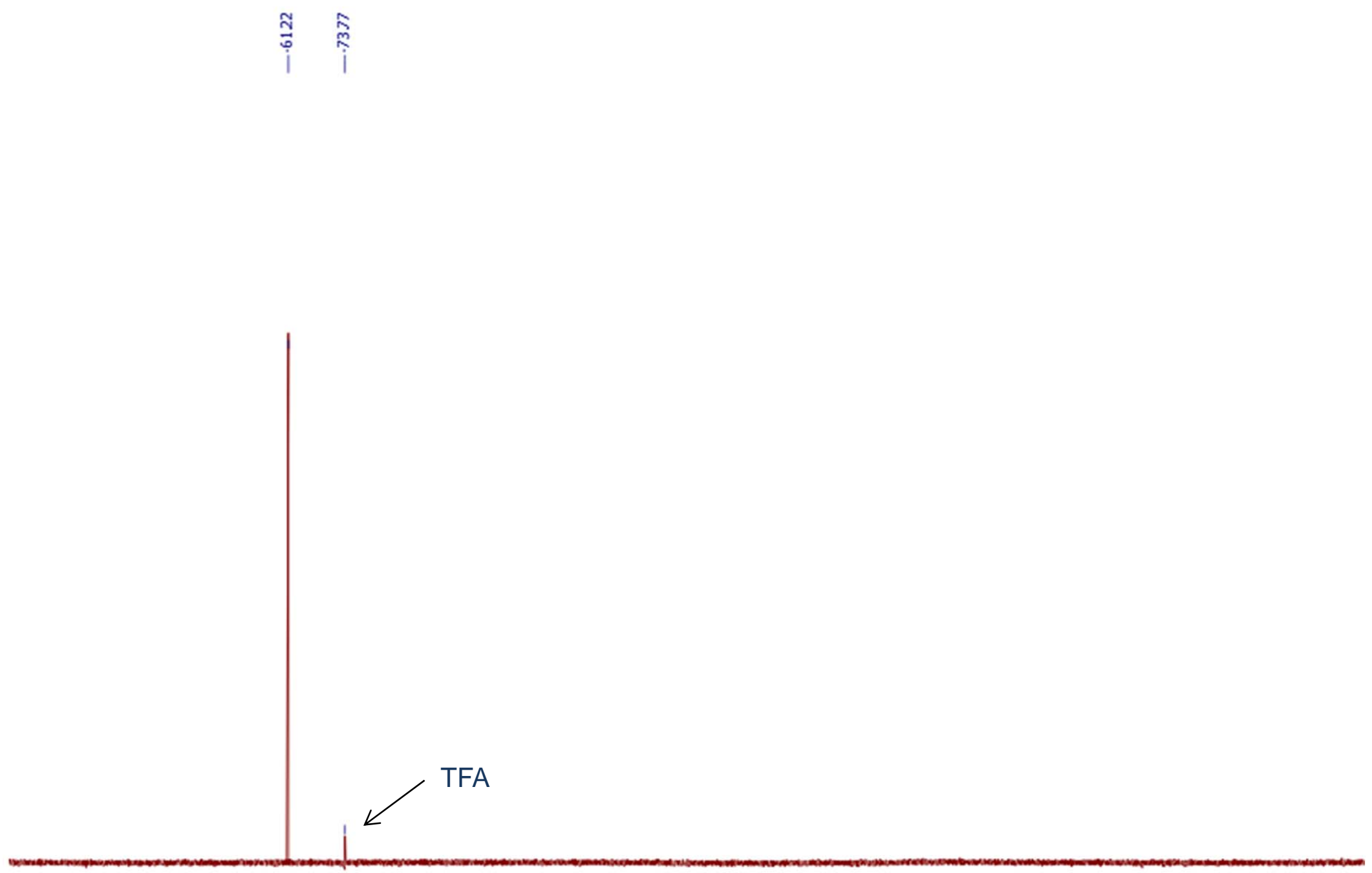

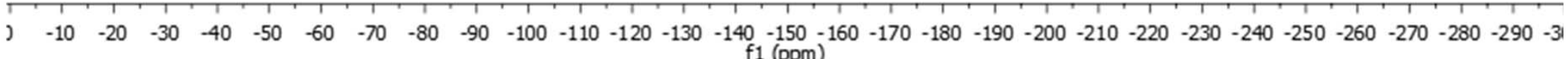


${ }^{1} \mathrm{H} /{ }^{1} \mathrm{H}$ COSY NMR spectrum of 5b•TFA $\left(600 / 600 \mathrm{MHz}\right.$, DMSO- $\left.\mathrm{d}_{6}\right)$

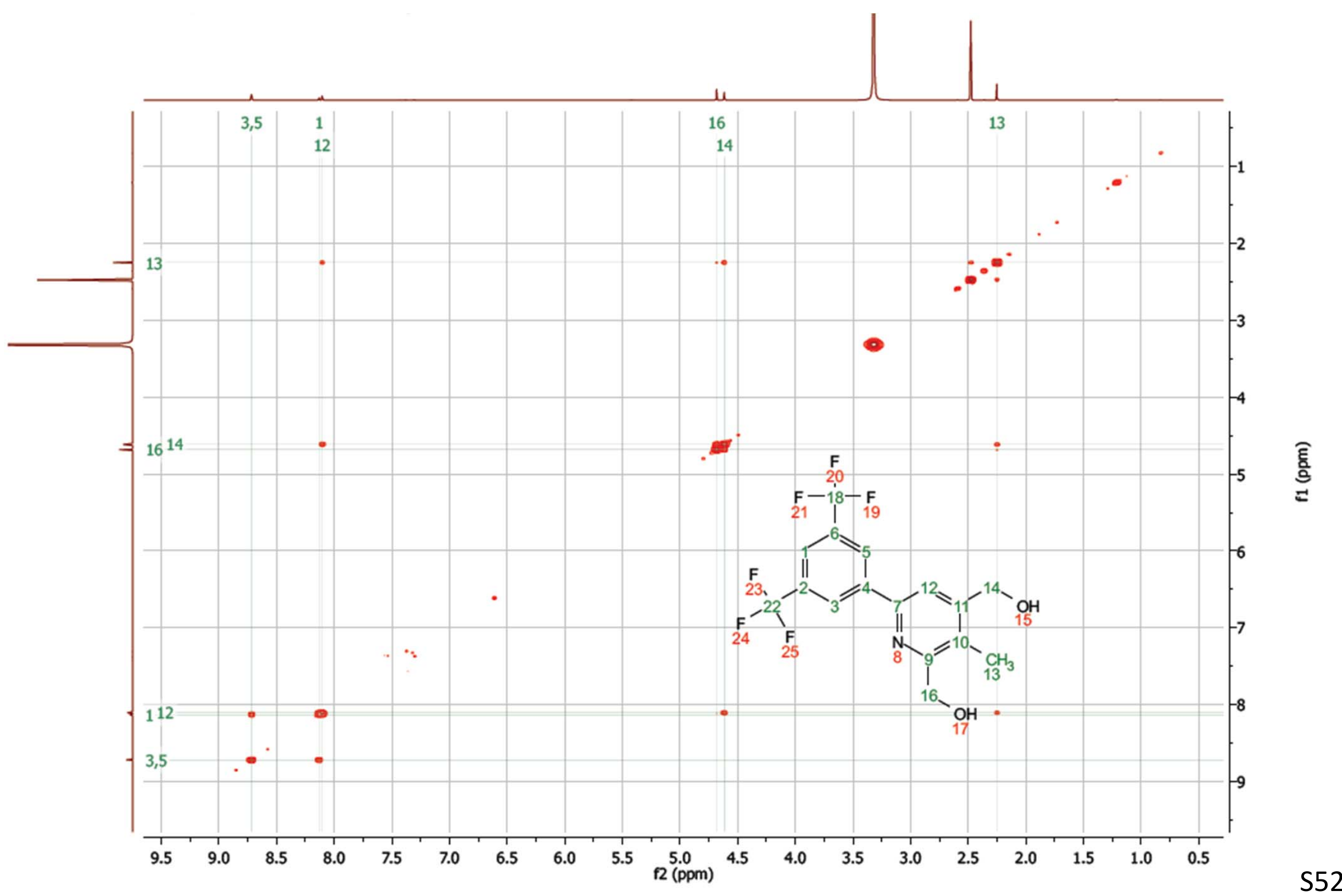


${ }^{1} \mathrm{H} /{ }^{13} \mathrm{C}$ HSQC NMR spectrum of 5b•TFA (600 / $150 \mathrm{MHz}$, DMSO- $\left.\mathrm{d}_{6}\right)$

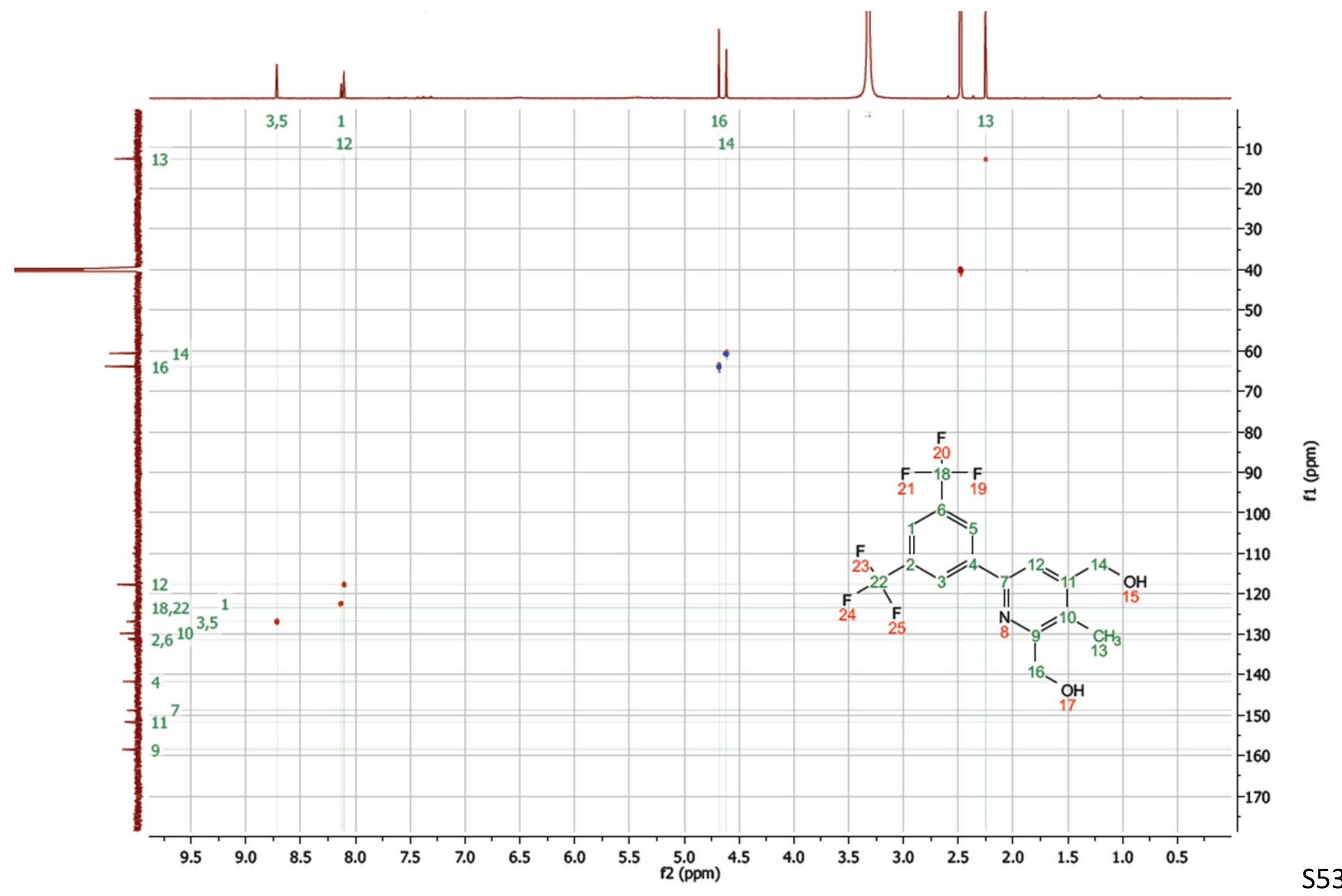


${ }^{1} \mathrm{H} /{ }^{13} \mathrm{C}$ HMBC NMR spectrum of 5b•TFA (600 / $150 \mathrm{MHz}$, DMSO-d $\left.{ }_{6}\right)$

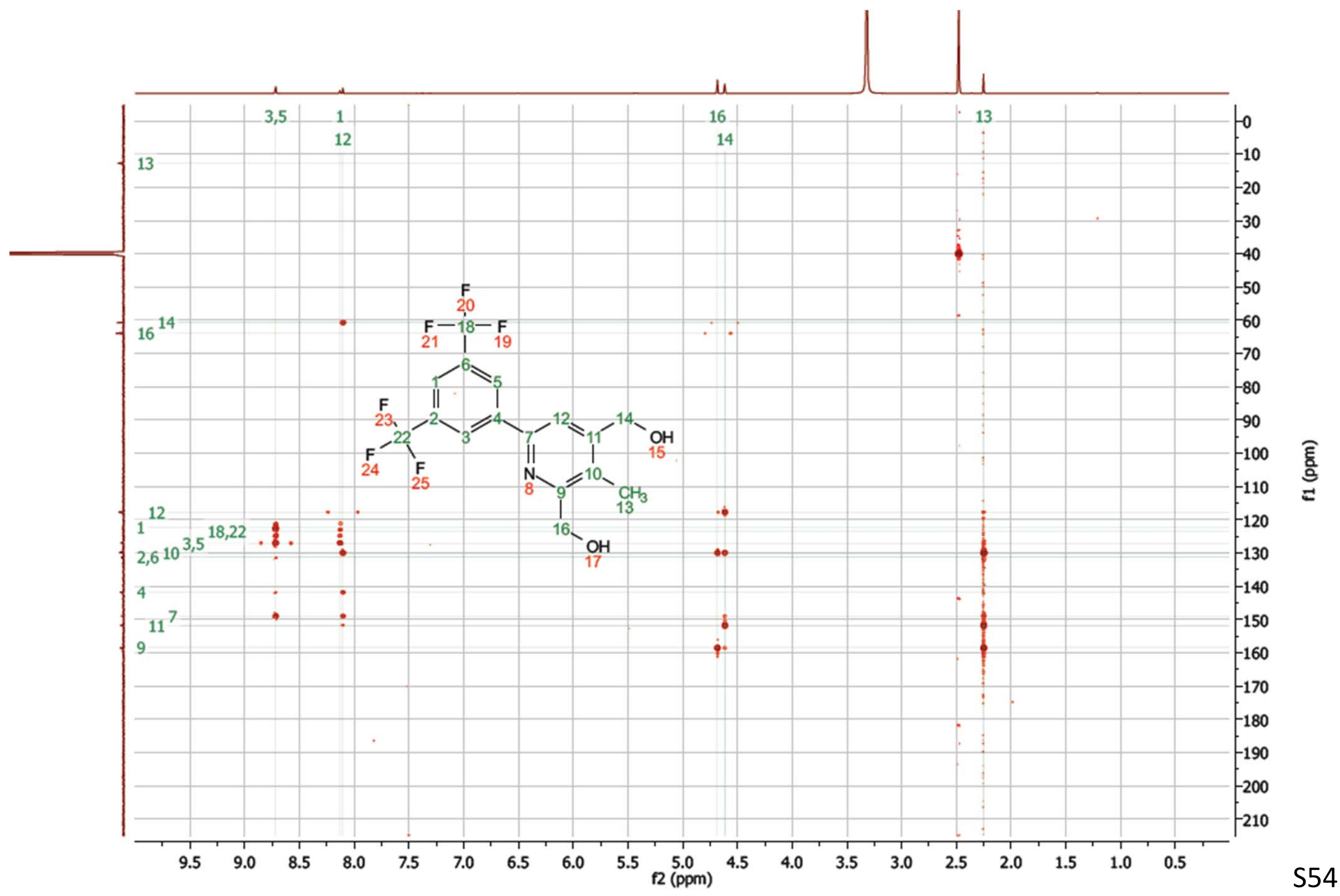


${ }^{1} \mathrm{H} /{ }^{1} \mathrm{H}$ ROESY NMR spectrum of 5b•TFA $\left(600 / 600 \mathrm{MHz}\right.$, DMSO- $\left.\mathrm{d}_{6}\right)$

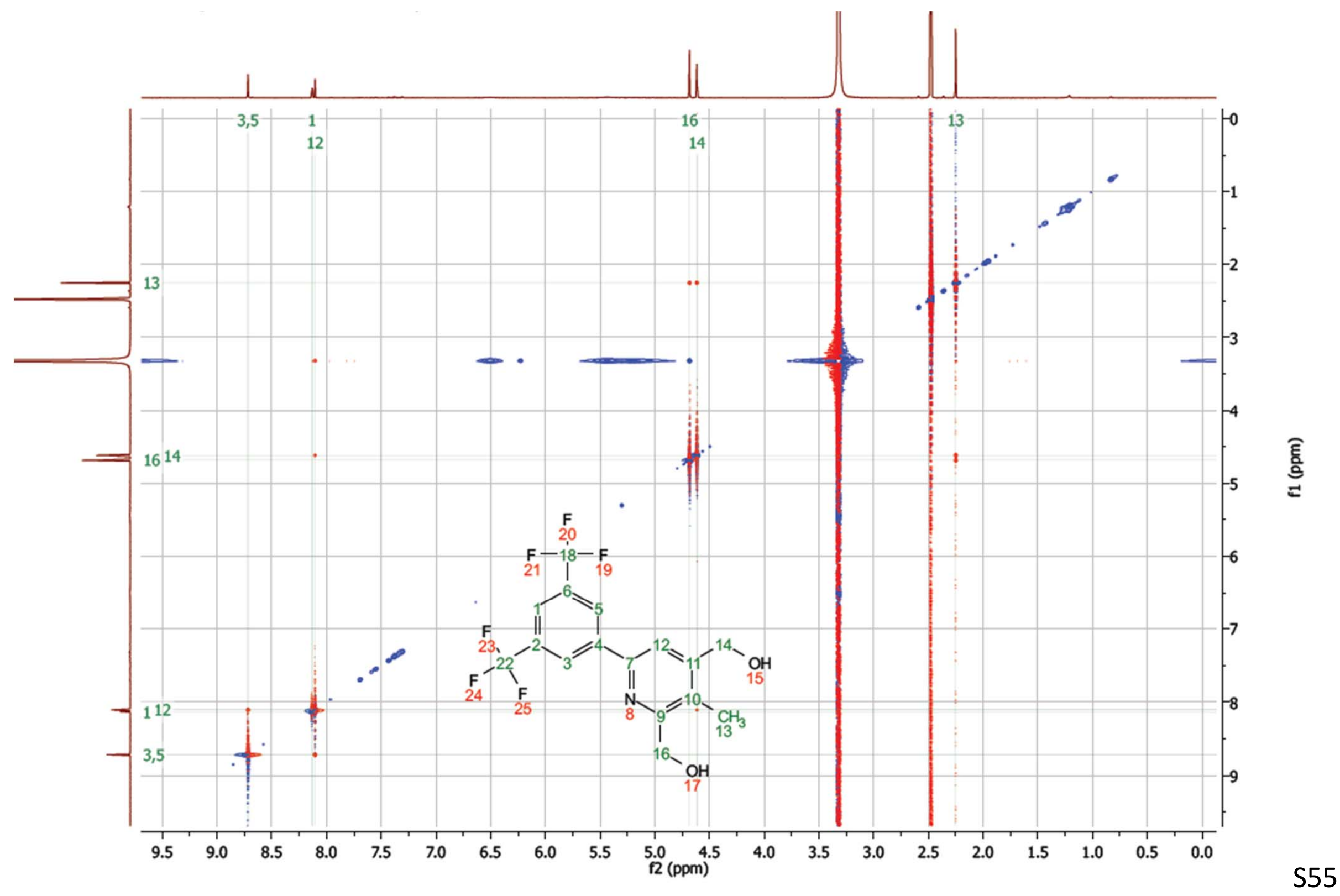


${ }^{1} \mathrm{H}$ NMR spectrum of $6 a \bullet T F A\left(600 \mathrm{MHz}\right.$, DMSO-d $\left.{ }_{6}\right)$
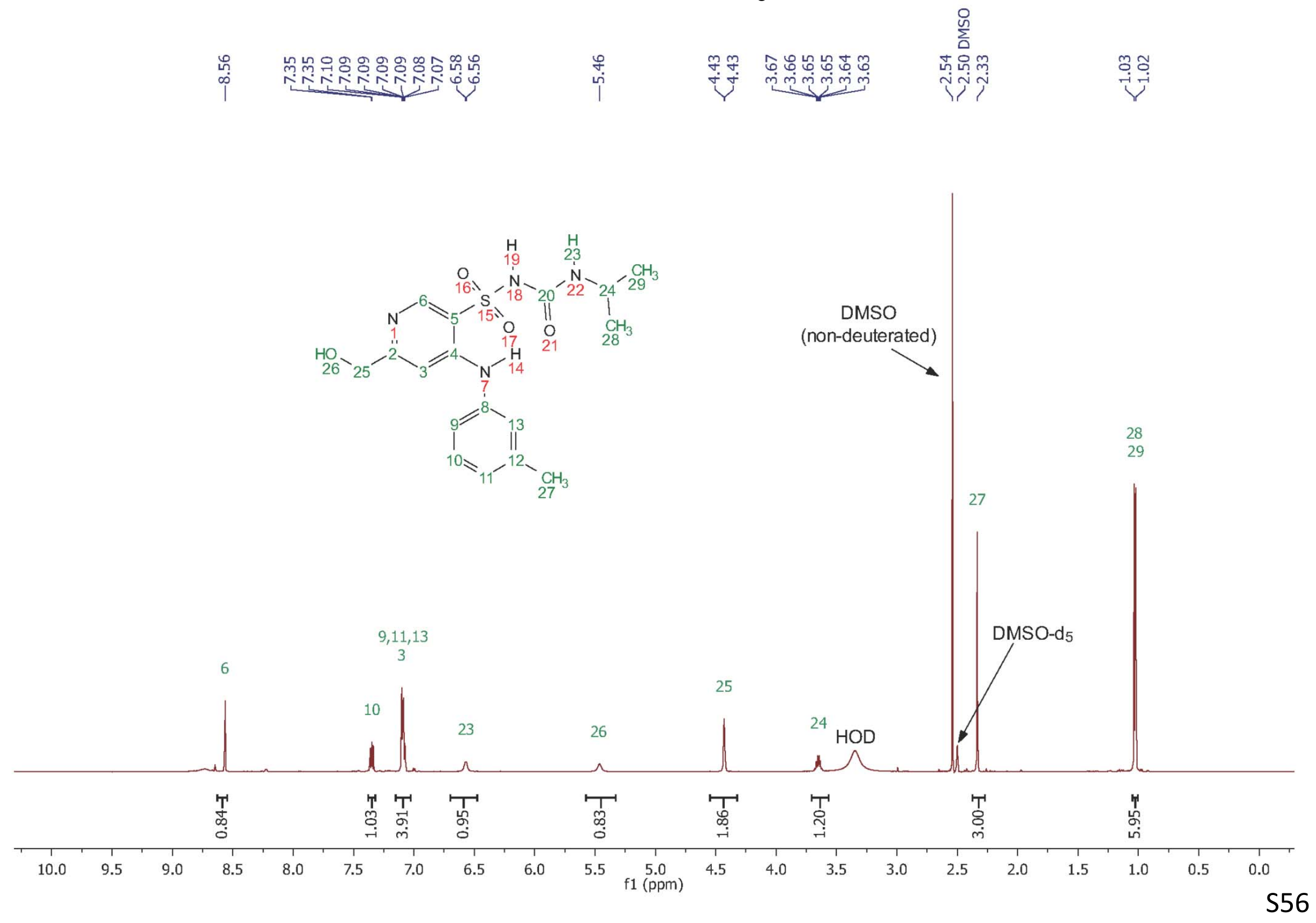
${ }^{13} \mathrm{C}$ NMR spectrum of $6 \mathbf{6} \bullet T F A\left(150 \mathrm{MHz}, \mathrm{DMSO}-\mathrm{d}_{6}\right)$
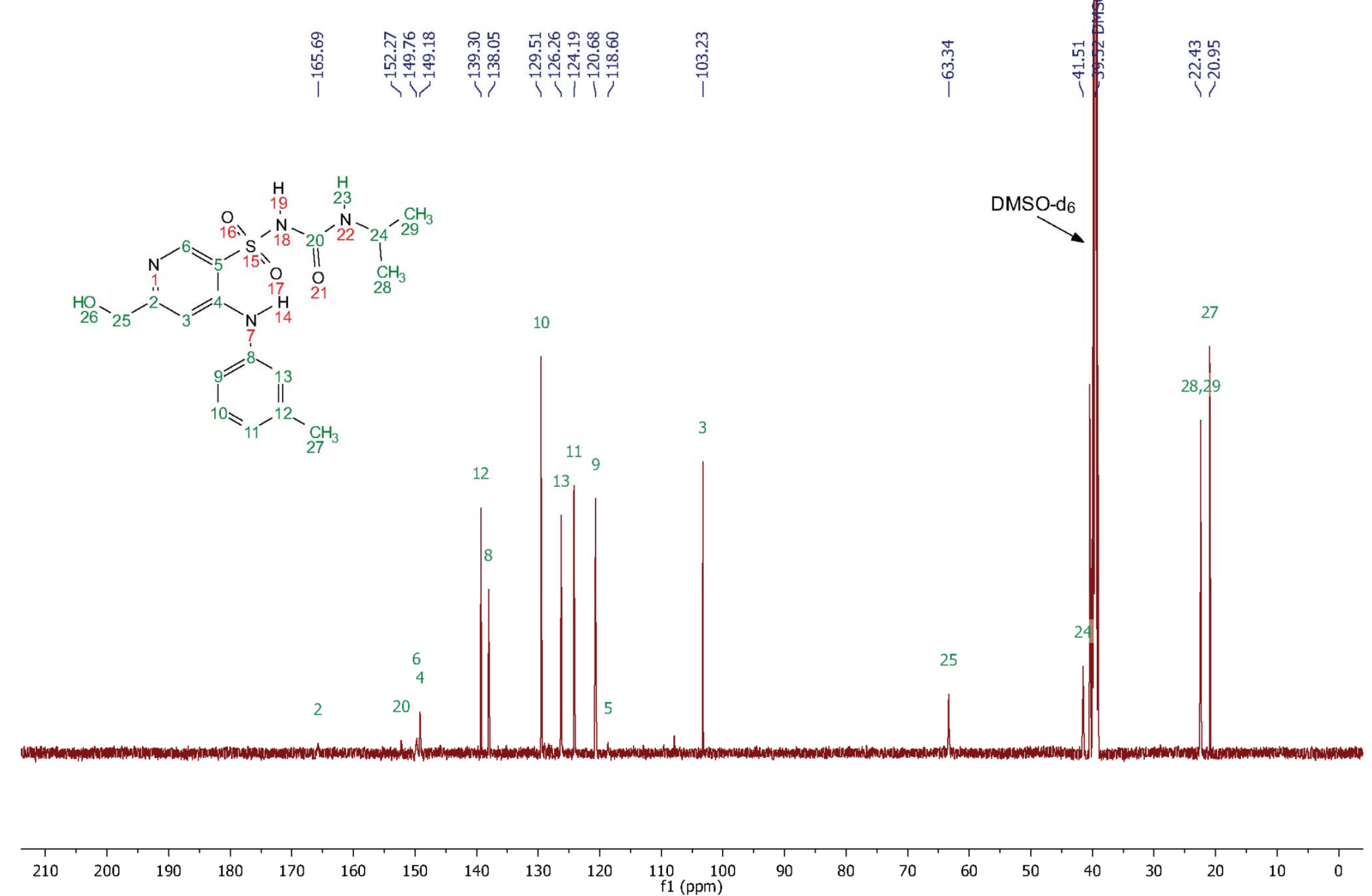
${ }^{19} \mathrm{~F}$ NMR spectrum of $6 \mathbf{a} \bullet T F A\left(470 \mathrm{MHz}, \mathrm{DMSO}-\mathrm{d}_{6}\right)$

î

TFA

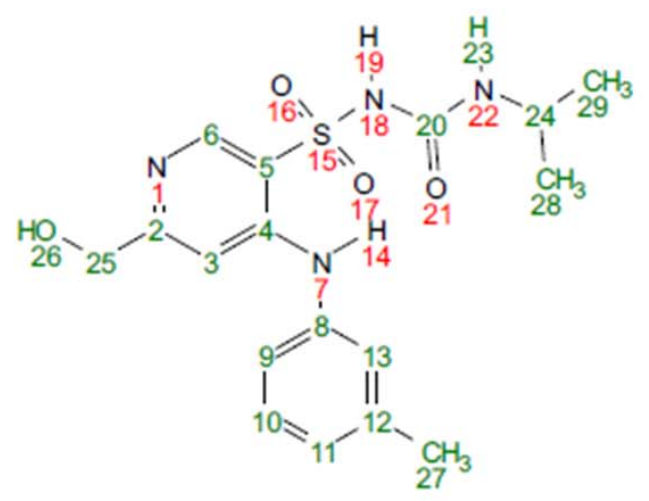

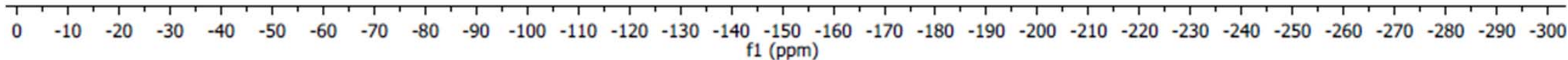


${ }^{1} \mathrm{H} /{ }^{1} \mathrm{H}$ COSY NMR spectrum of $6 a \bullet T F A\left(600 / 600 \mathrm{MHz}\right.$, DMSO- $\left.\mathrm{d}_{6}\right)$

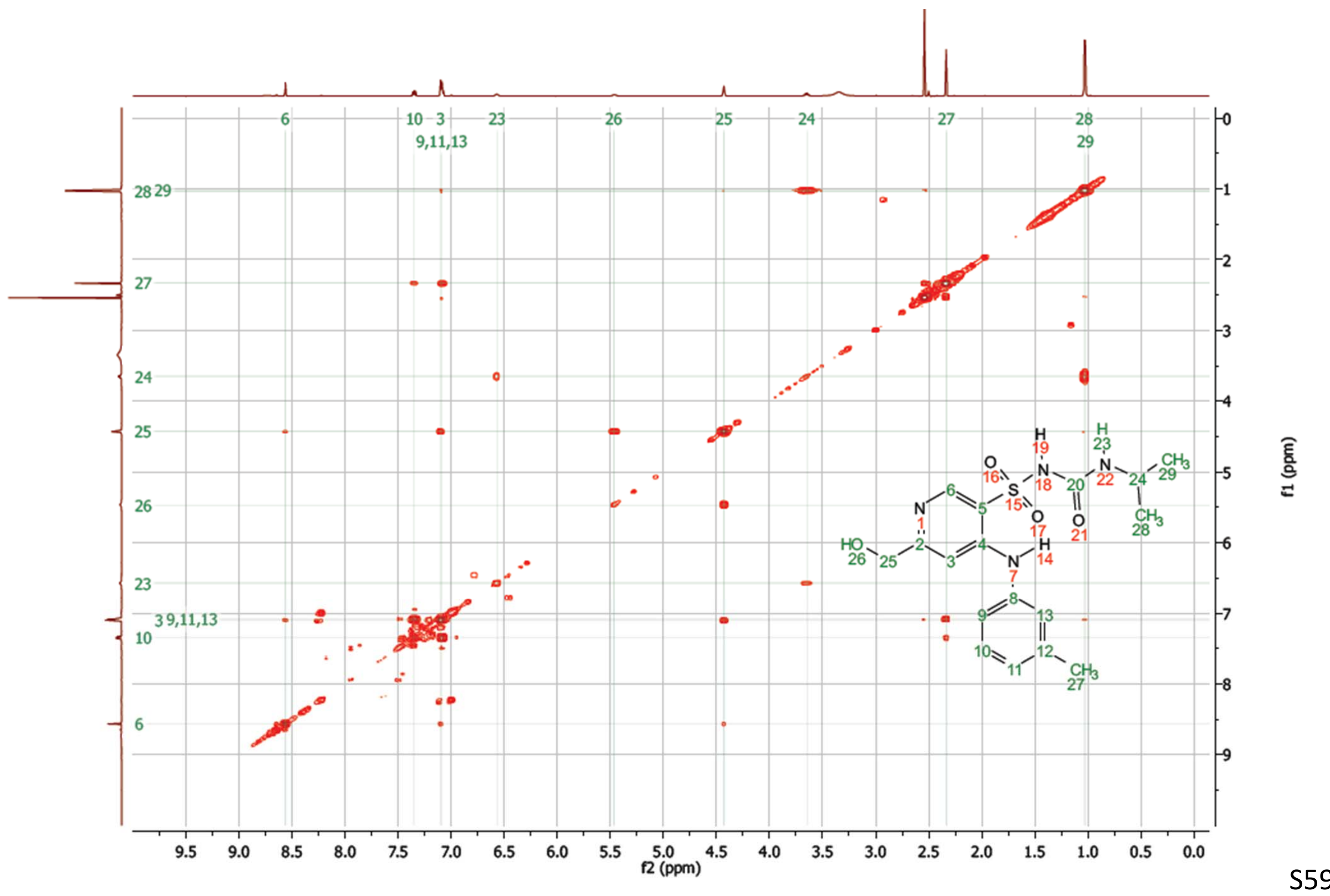


${ }^{1} \mathrm{H} /{ }^{13} \mathrm{C}$ HSQC NMR spectrum of 6a•TFA $\left(600 / 150 \mathrm{MHz}\right.$, DMSO-d $\left.{ }_{6}\right)$

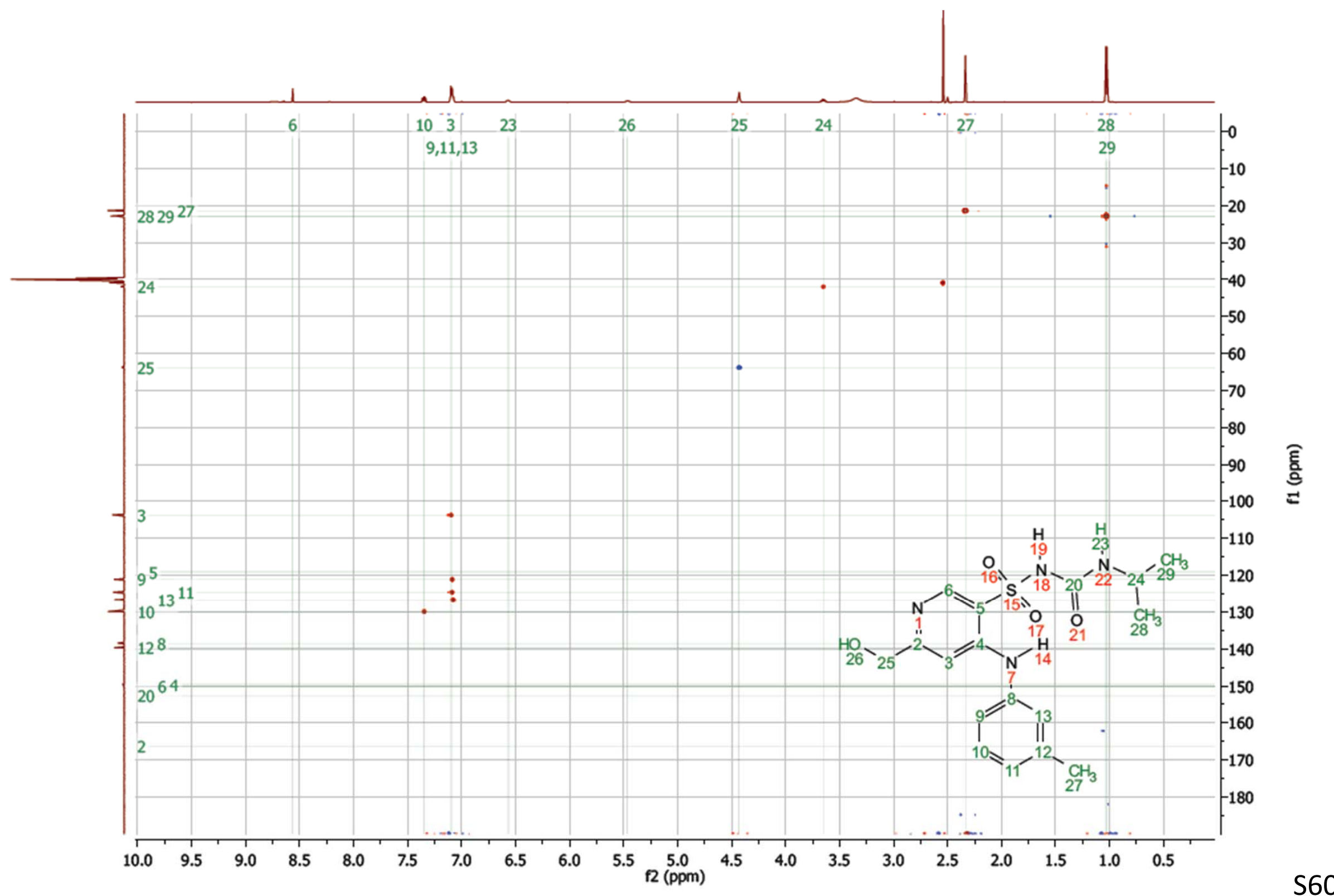


${ }^{1} \mathrm{H} /{ }^{13} \mathrm{C}$ HMBC NMR spectrum of 6a•TFA (600 / $150 \mathrm{MHz}$, DMSO-d $\mathrm{d}_{6}$ )

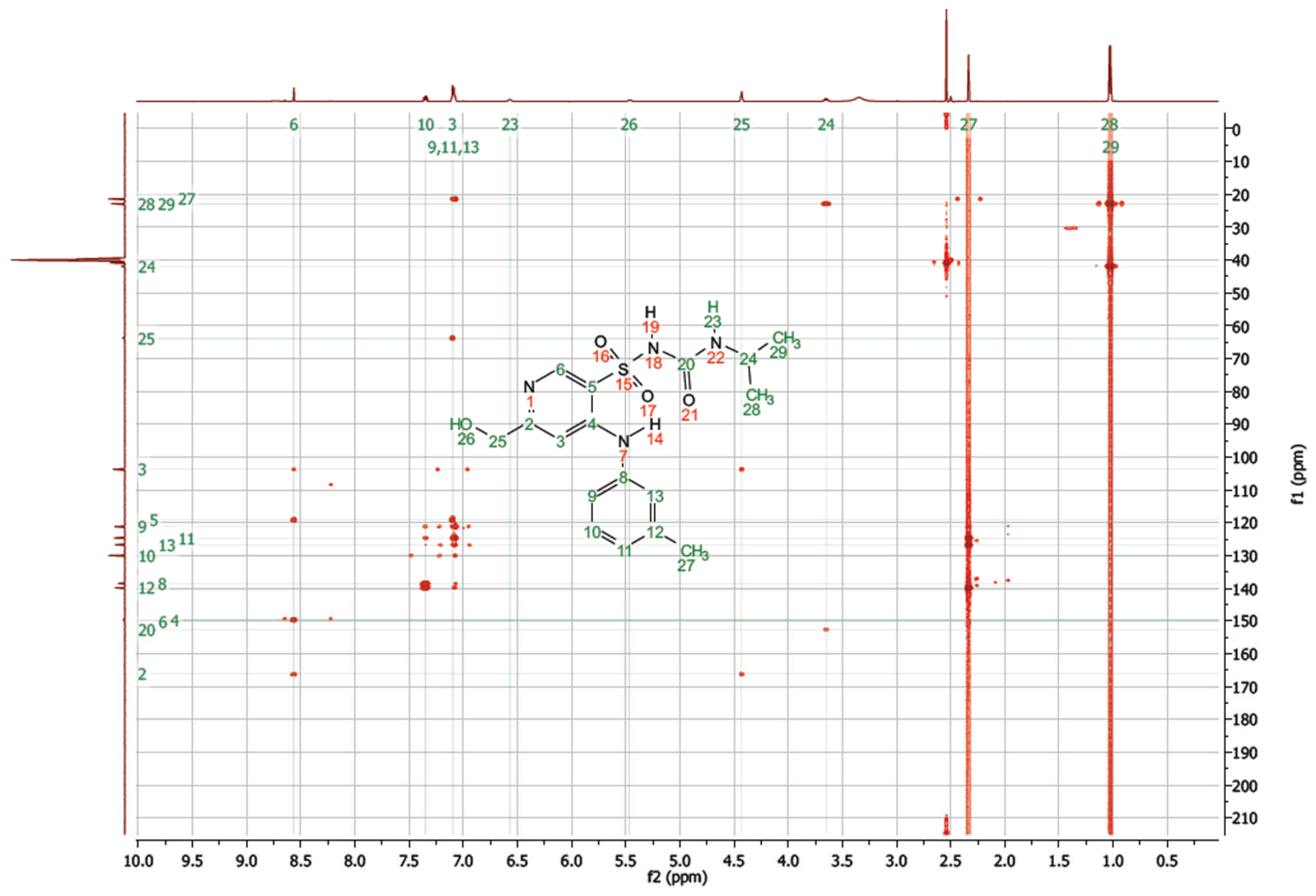


${ }^{1} \mathrm{H}$ NMR spectrum of $7 \mathrm{a}\left(500 \mathrm{MHz}, \mathrm{DMSO}-\mathrm{d}_{6}\right)$

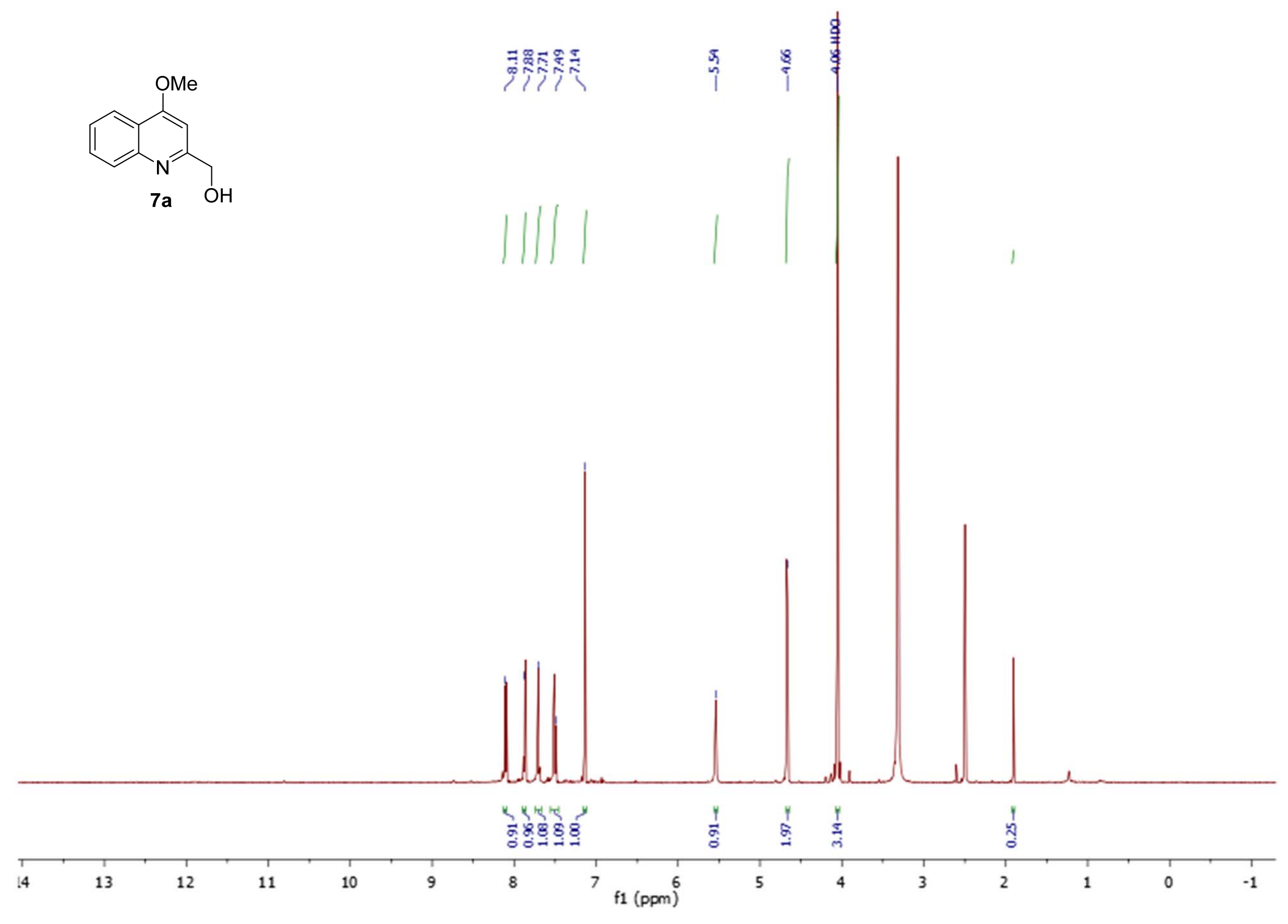


${ }^{13} \mathrm{C}$ NMR spectrum of $7 \mathbf{a}\left(125 \mathrm{MHz}, \mathrm{DMSO}-\mathrm{d}_{6}\right)$

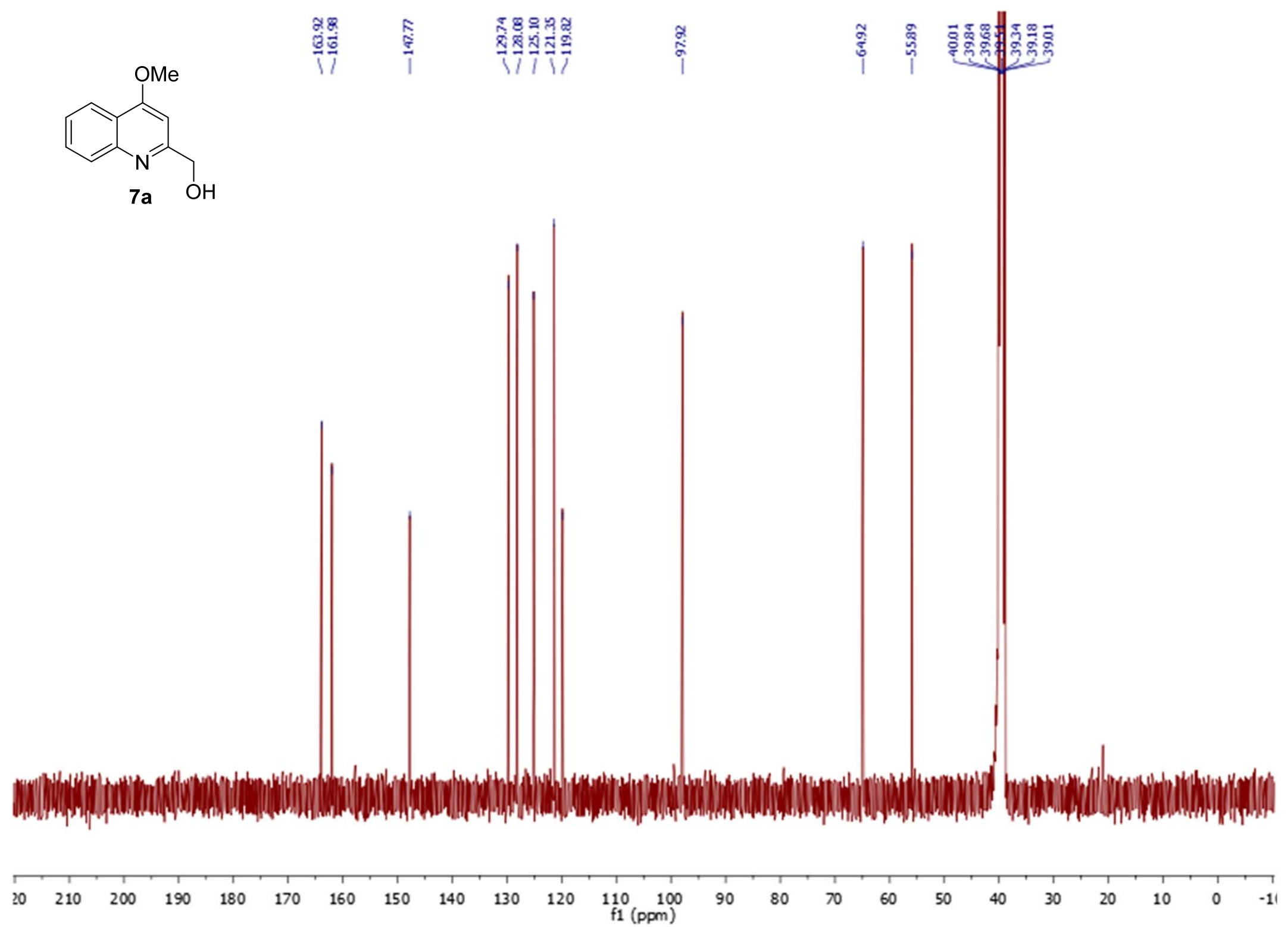


${ }^{1} \mathrm{H}$ NMR spectrum of $\mathbf{8 a}\left(500 \mathrm{MHz}, \mathrm{CD}_{3} \mathrm{OD}\right)$

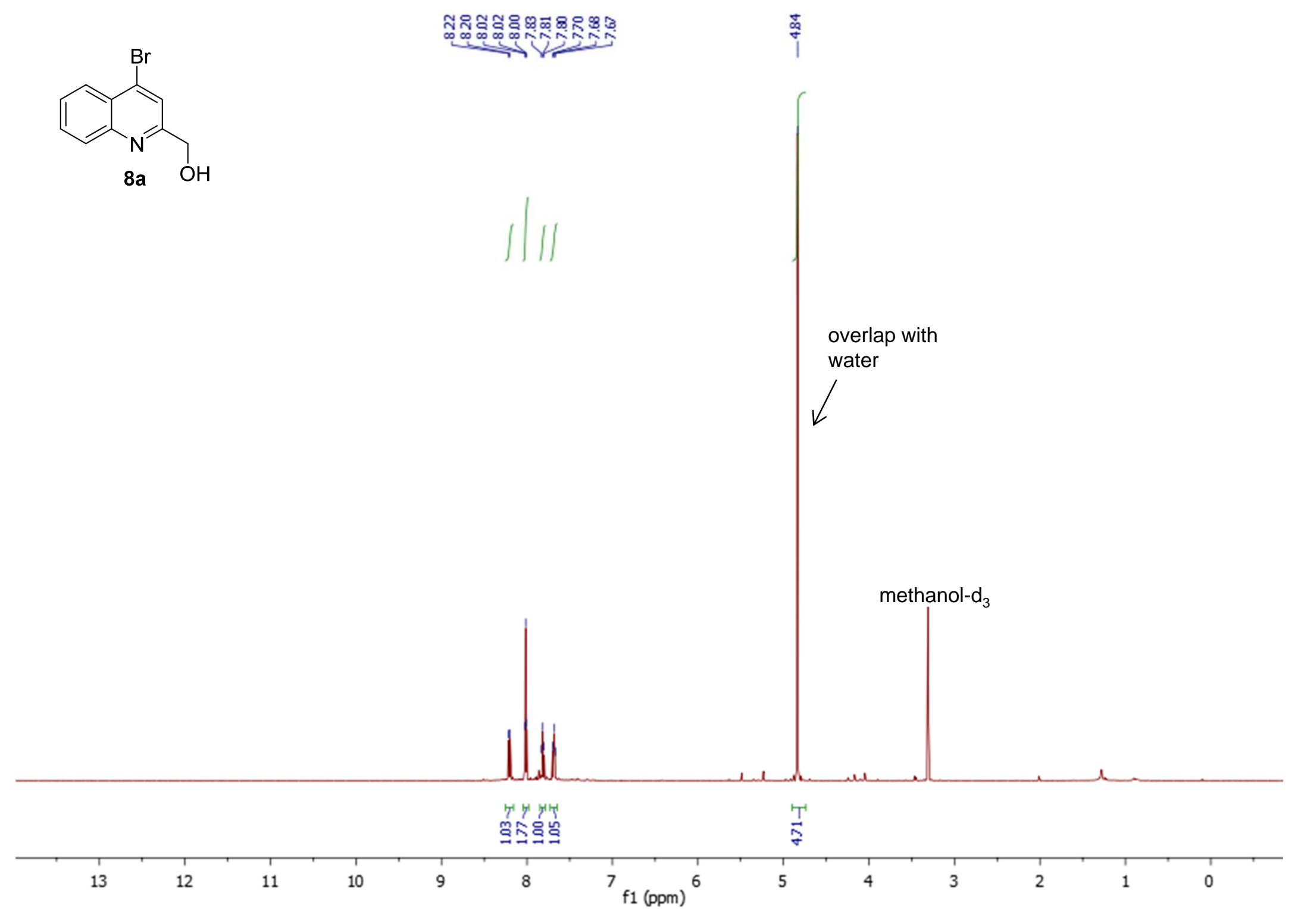


${ }^{13} \mathrm{C}$ NMR spectrum of $\mathbf{8 a}\left(125 \mathrm{MHz}, \mathrm{CD}_{3} \mathrm{OD}\right)$
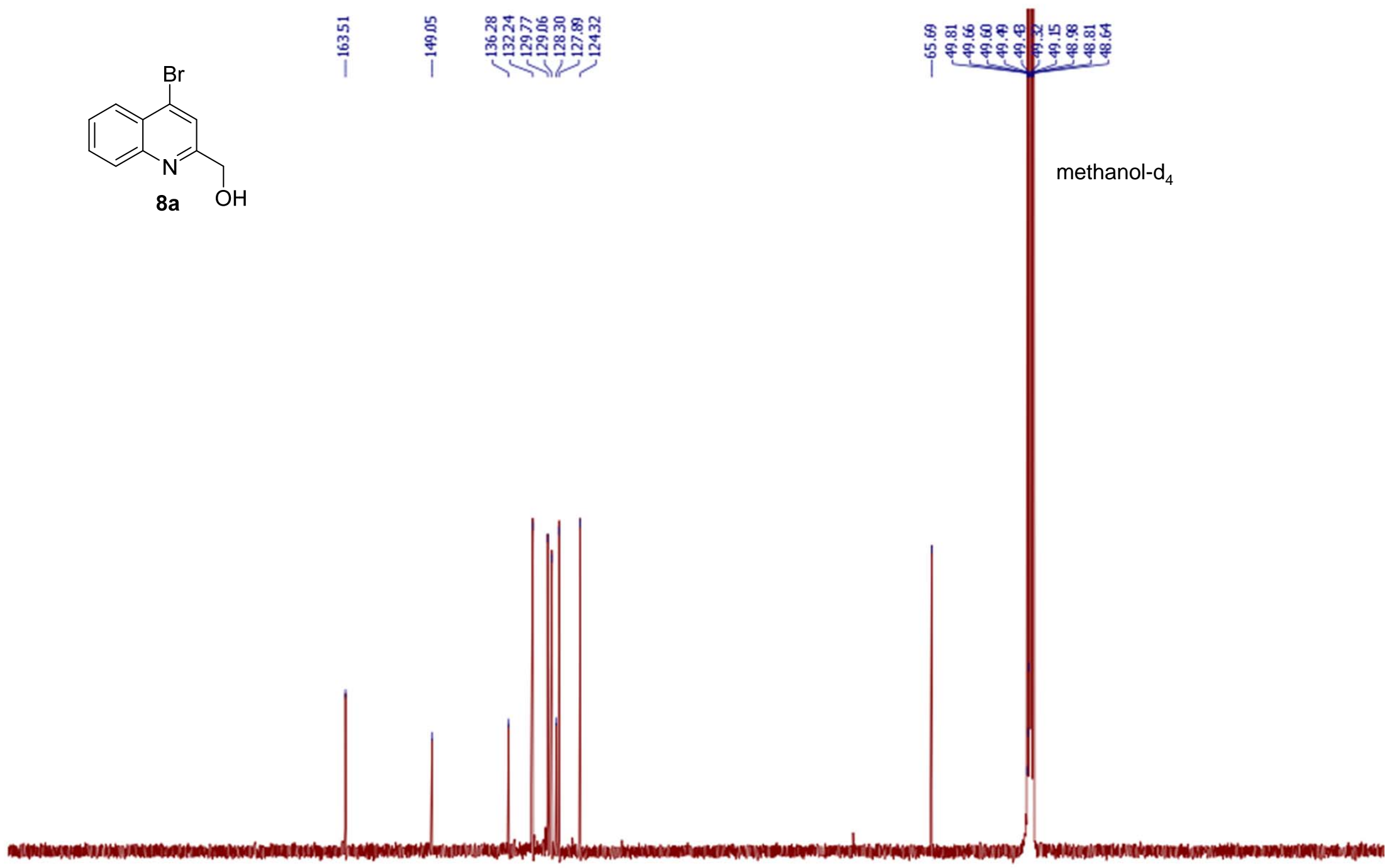

210200 
${ }^{1} \mathrm{H}$ NMR spectrum of $\mathbf{9 a}\left(500 \mathrm{MHz}, \mathrm{CD}_{3} \mathrm{OD}\right)$

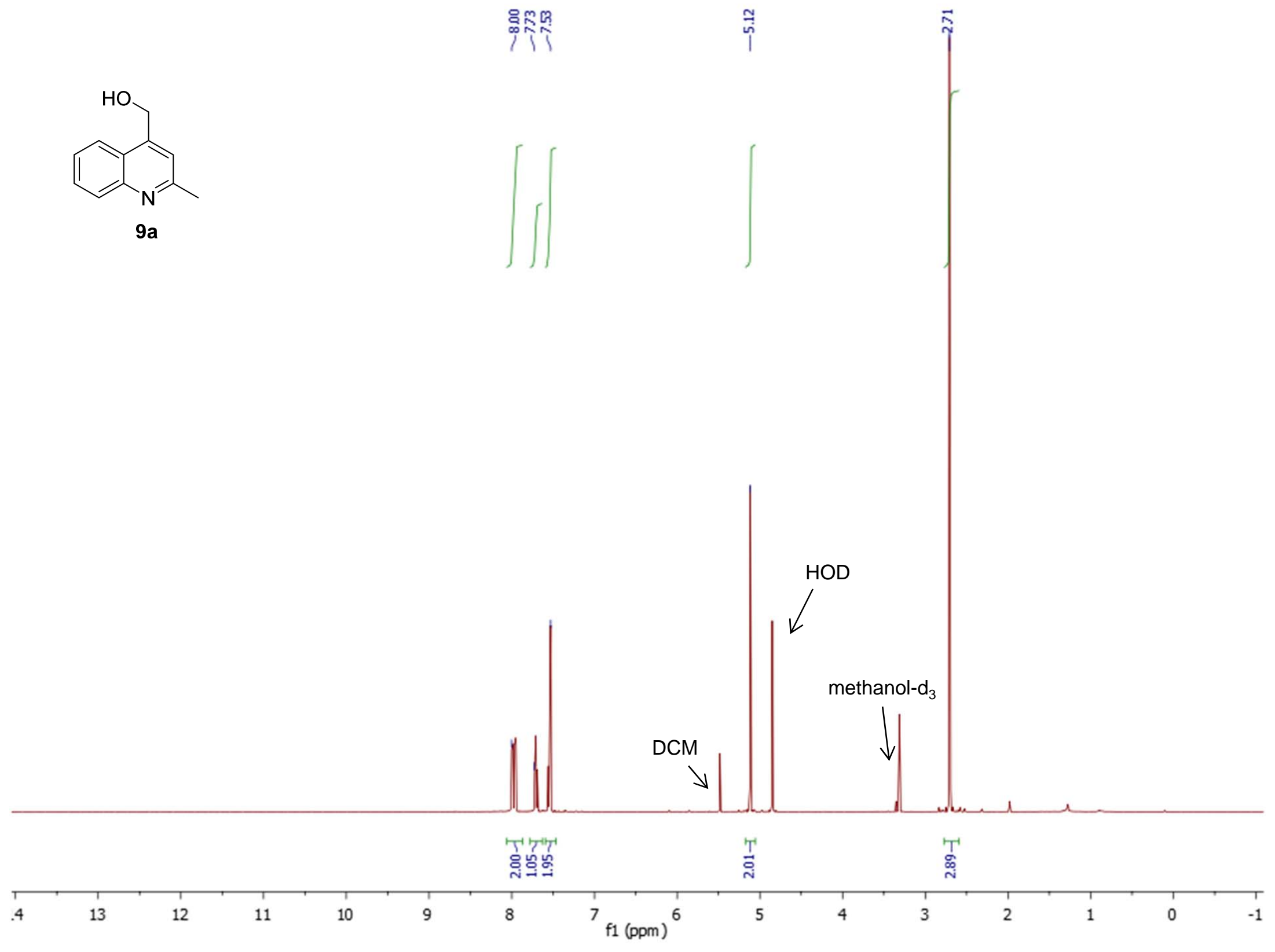


${ }^{13} \mathrm{C}$ NMR spectrum of $\mathbf{9 a}\left(125 \mathrm{MHz}, \mathrm{CD}_{3} \mathrm{OD}\right)$

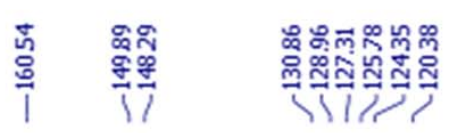

|
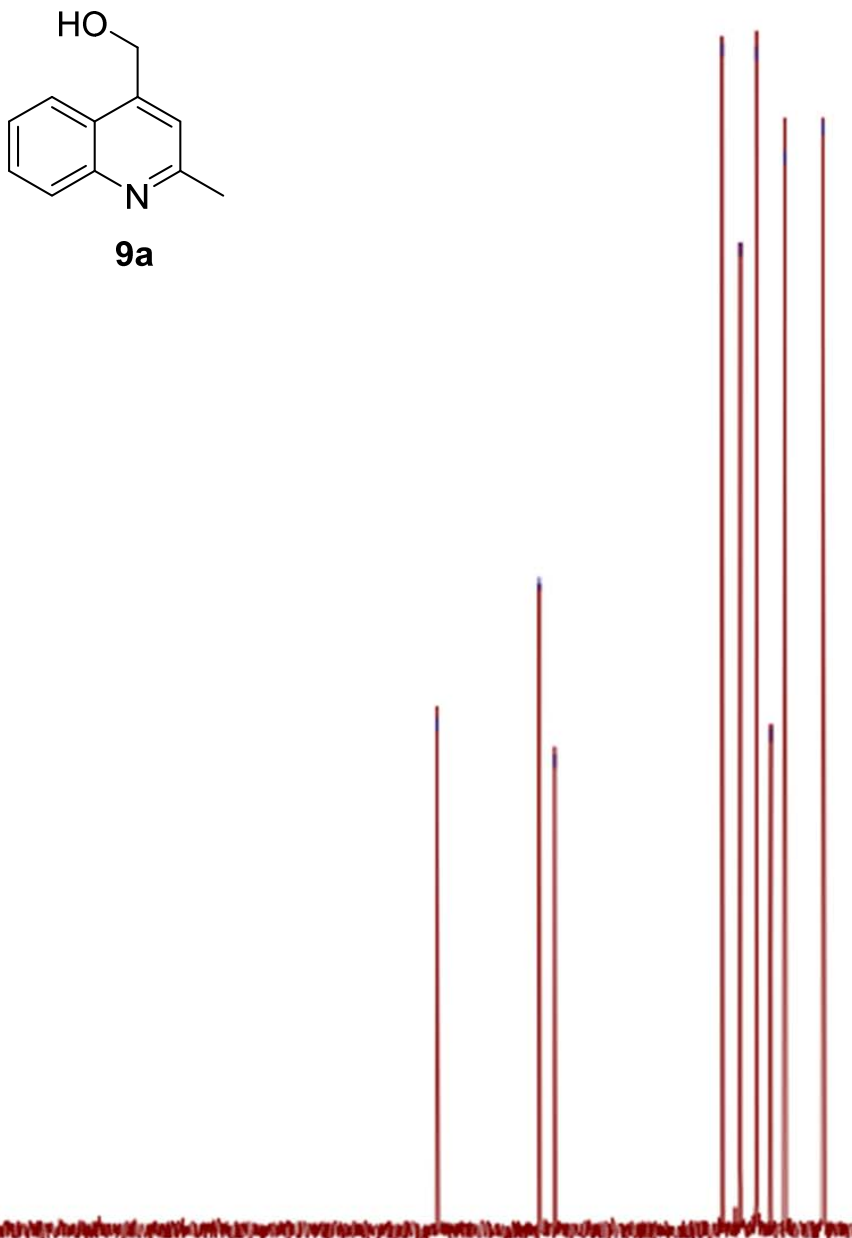

methanol- $\mathrm{d}_{4}$

$9 a$ 
${ }^{1} \mathrm{H}$ NMR spectrum of $10 \mathrm{a}\left(600 \mathrm{MHz}\right.$, DMSO-d $\left.\mathrm{d}_{6}\right)$

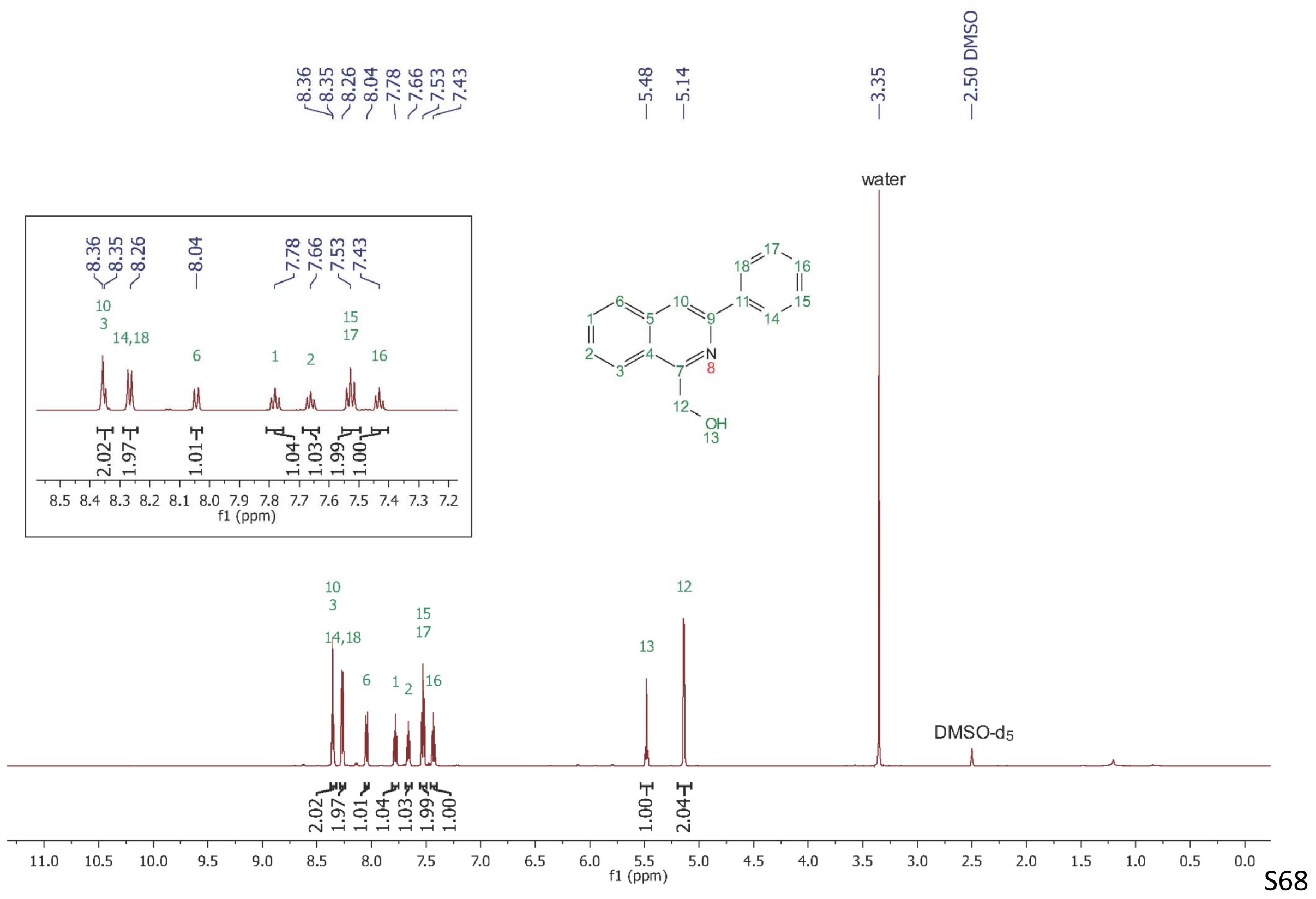


${ }^{13} \mathrm{C}$ NMR spectrum of $10 \mathrm{a}\left(150 \mathrm{MHz}, \mathrm{DMSO}-\mathrm{d}_{6}\right)$
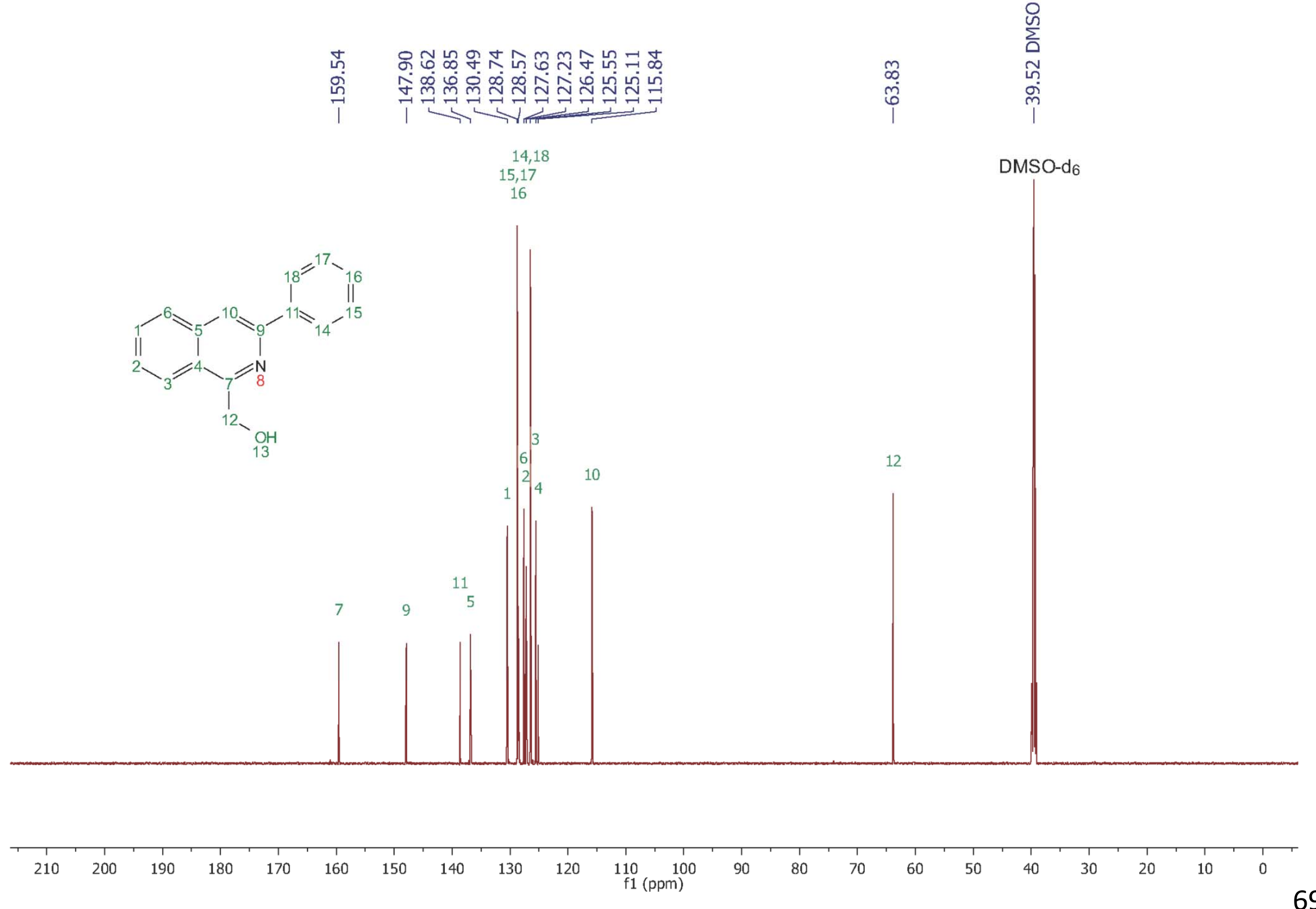
${ }^{1} \mathrm{H} /{ }^{1} \mathrm{H}$ COSY NMR spectrum of $10 a\left(600 / 600 \mathrm{MHz}\right.$, DMSO-d $\left.{ }_{6}\right)$

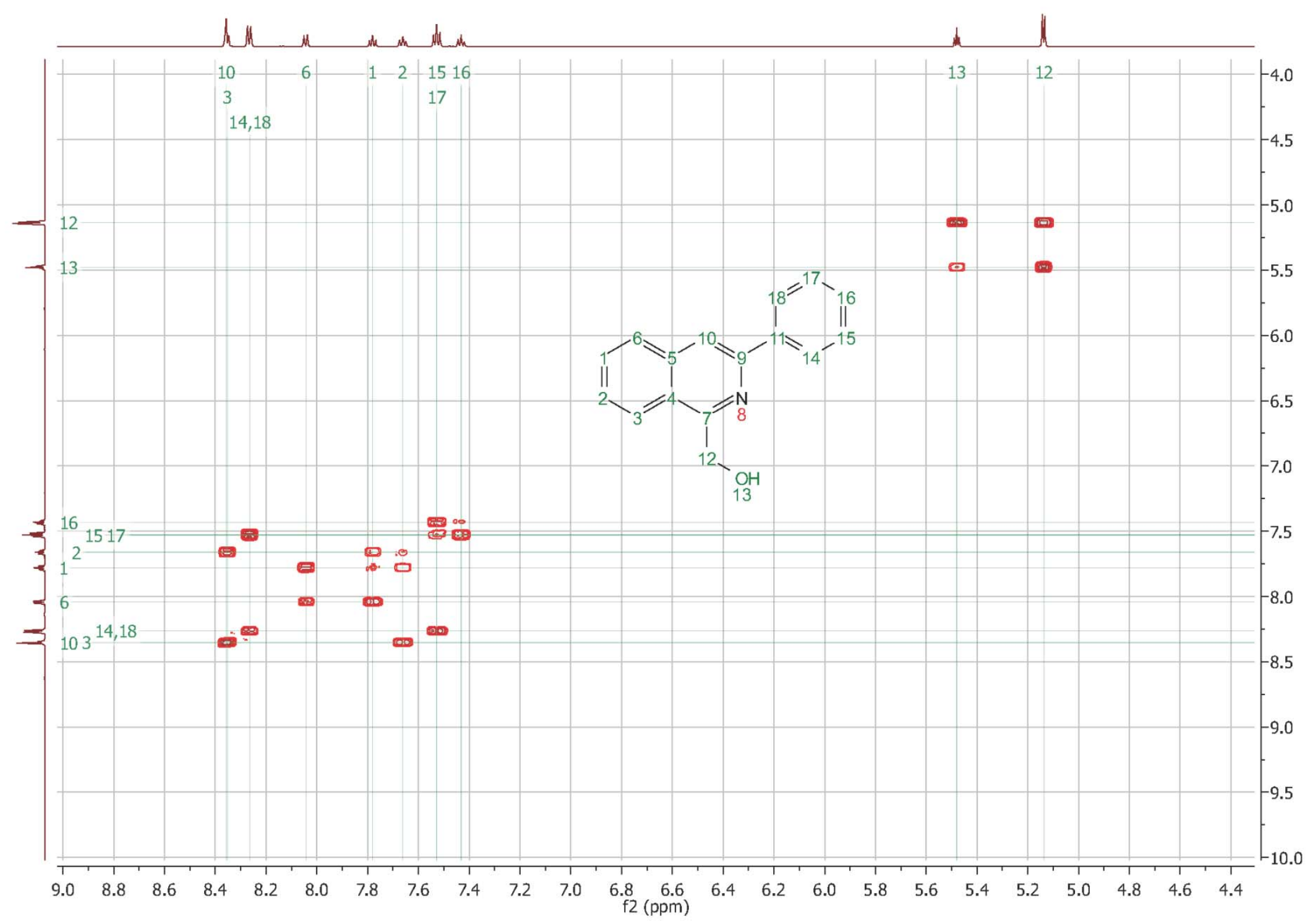


${ }^{1} \mathrm{H} /{ }^{13} \mathrm{C}$ HSQC NMR spectrum of $10 \mathrm{a}\left(600 / 150 \mathrm{MHz}\right.$, DMSO-d $\left.\mathrm{d}_{6}\right)$

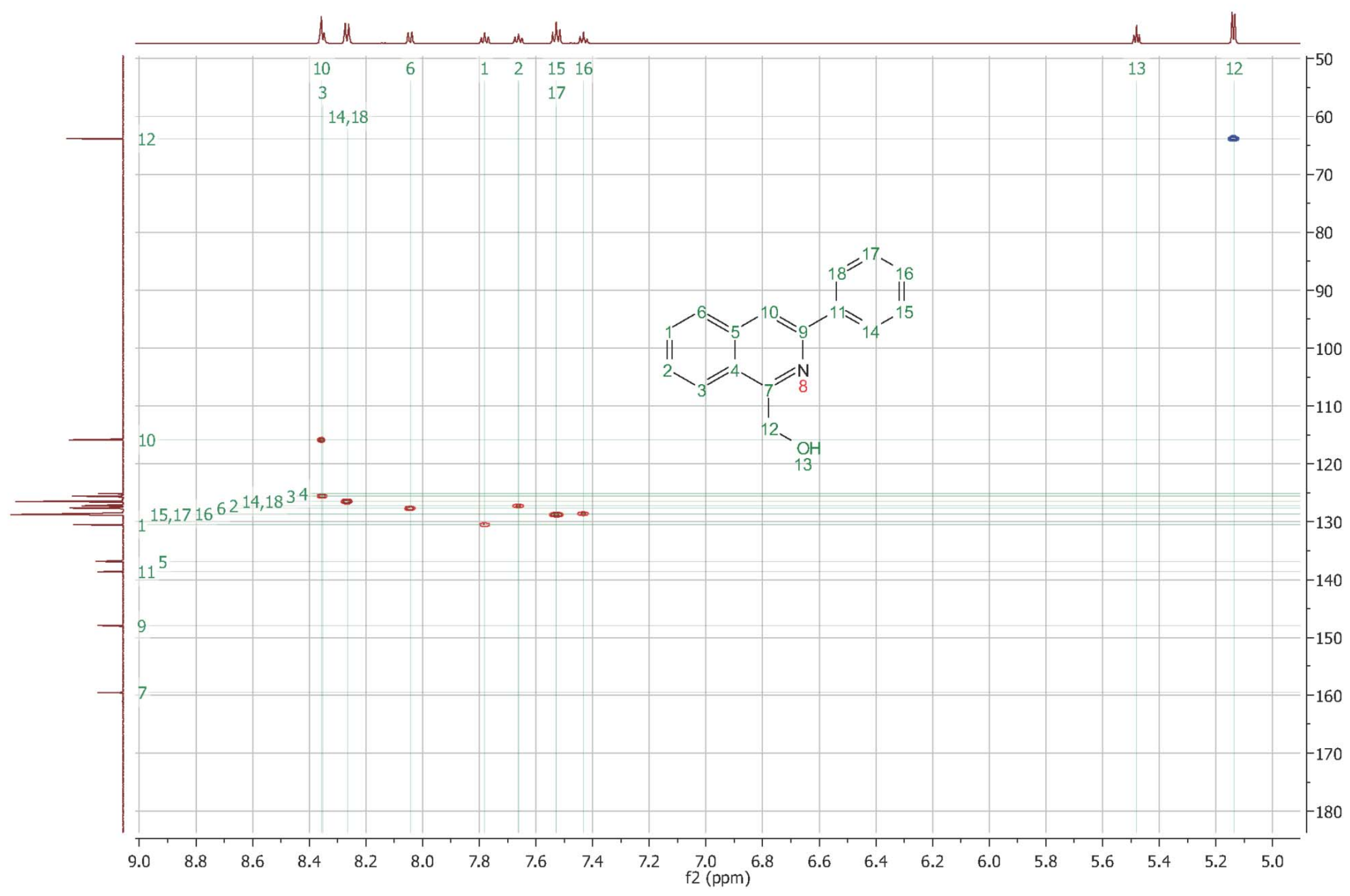


${ }^{1} \mathrm{H} /{ }^{13} \mathrm{C}$ HSQC-TOCSY NMR spectrum of $10 a\left(600 / 150 \mathrm{MHz}\right.$, DMSO-d $\left.\mathrm{d}_{6}\right)$

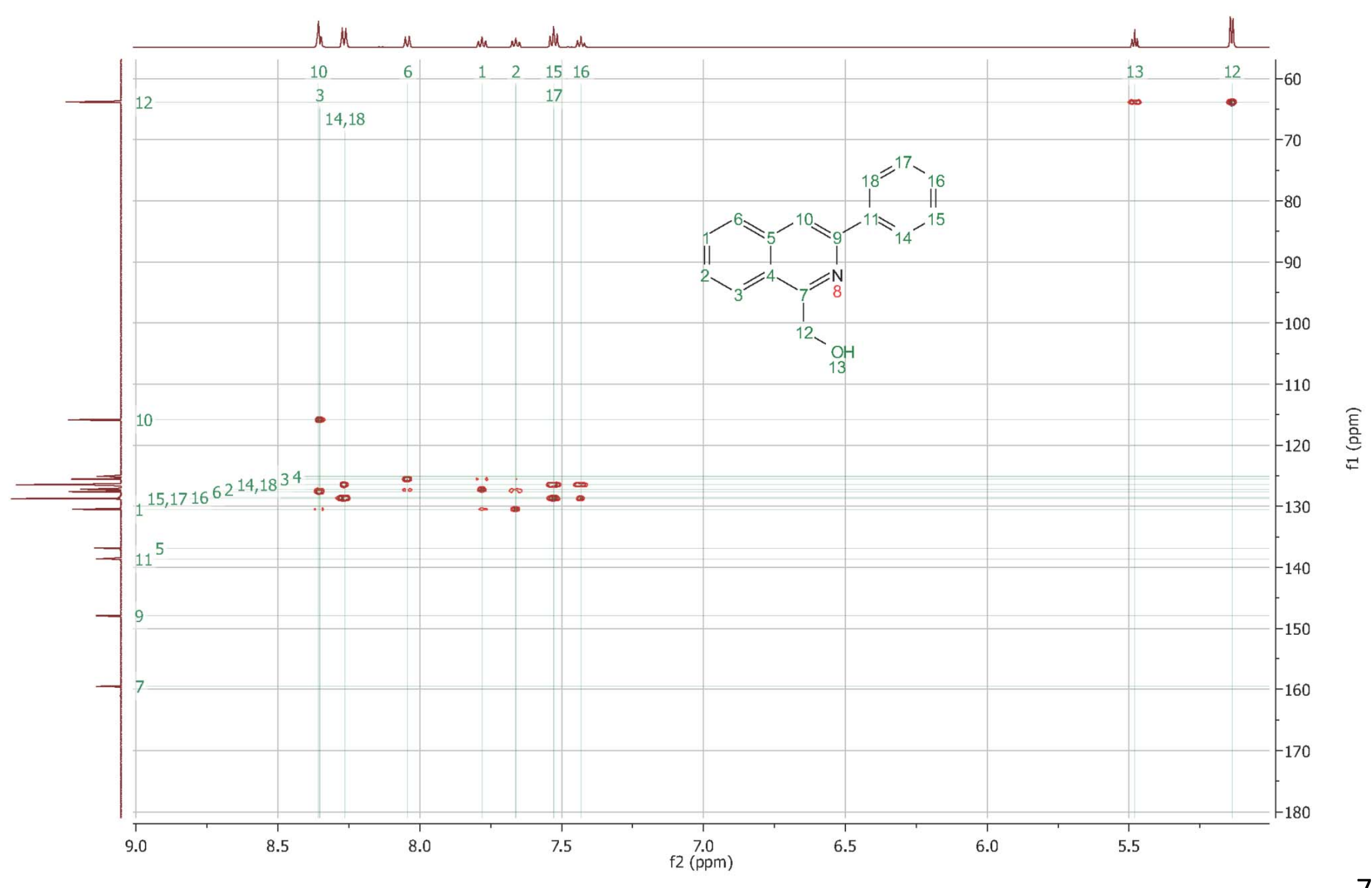


${ }^{1} \mathrm{H} /{ }^{13} \mathrm{C}$ HMBC NMR spectrum of 10a (600 / $150 \mathrm{MHz}$, DMSO-d $\left.\mathrm{d}_{6}\right)$

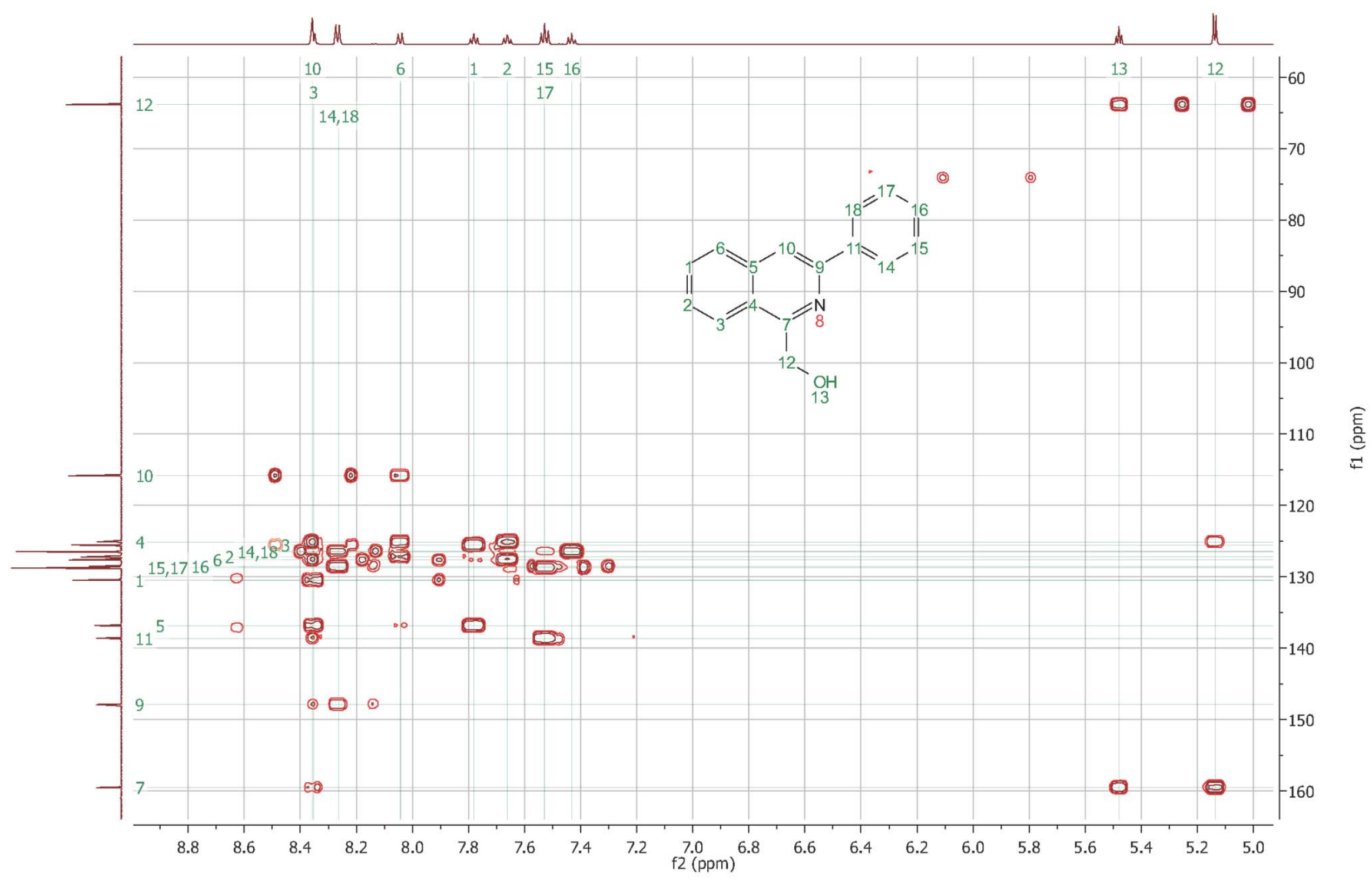


${ }^{1} \mathrm{H} /{ }^{1} \mathrm{H}$ ROESY NMR spectrum of $10 a\left(600 / 600 \mathrm{MHz}\right.$, DMSO-d $\left.{ }_{6}\right)$

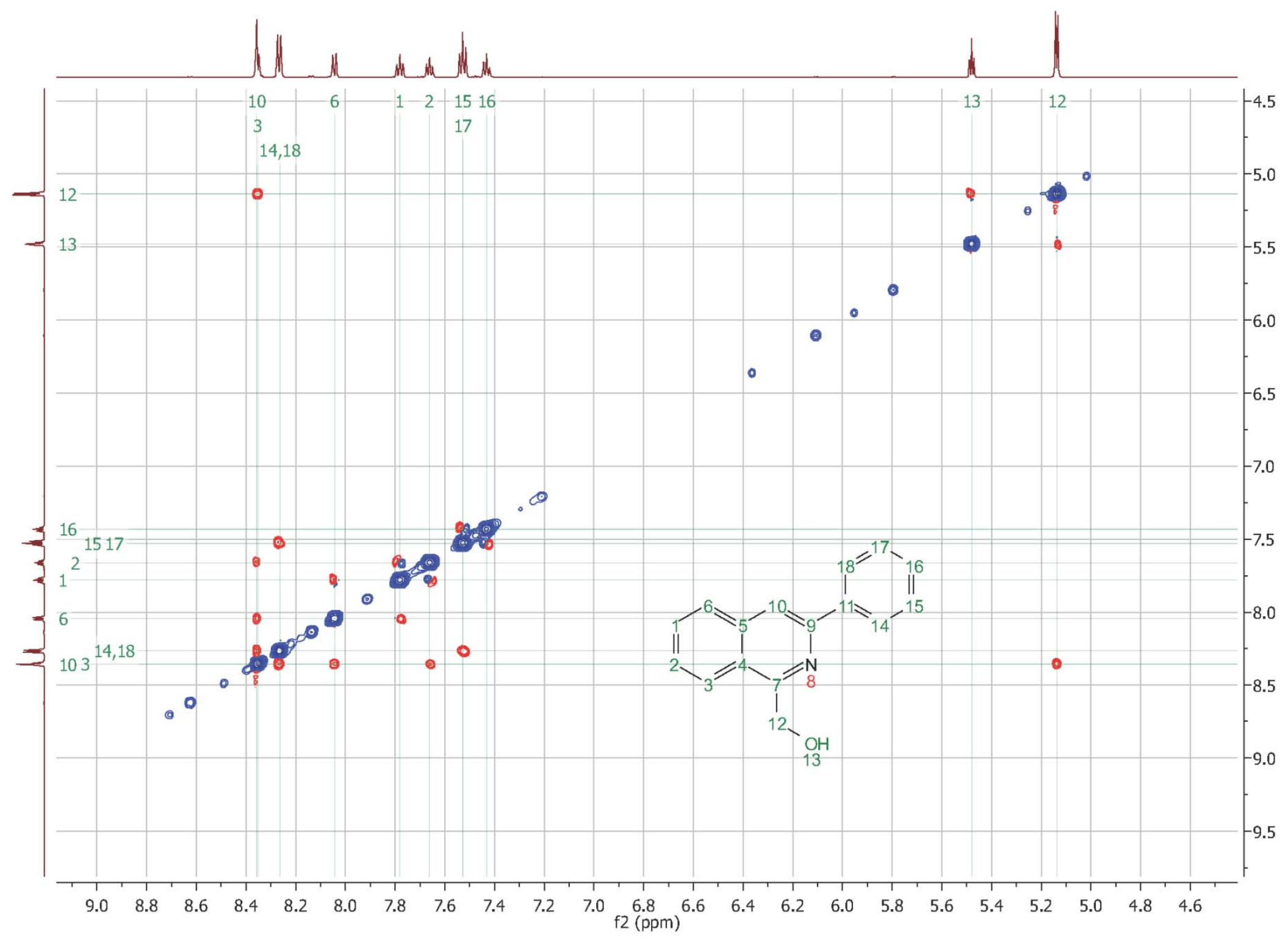


${ }^{1} \mathrm{H}$ NMR spectrum of $10 \mathrm{~b}\left(600 \mathrm{MHz}, \mathrm{DMSO}-\mathrm{d}_{6}\right)$

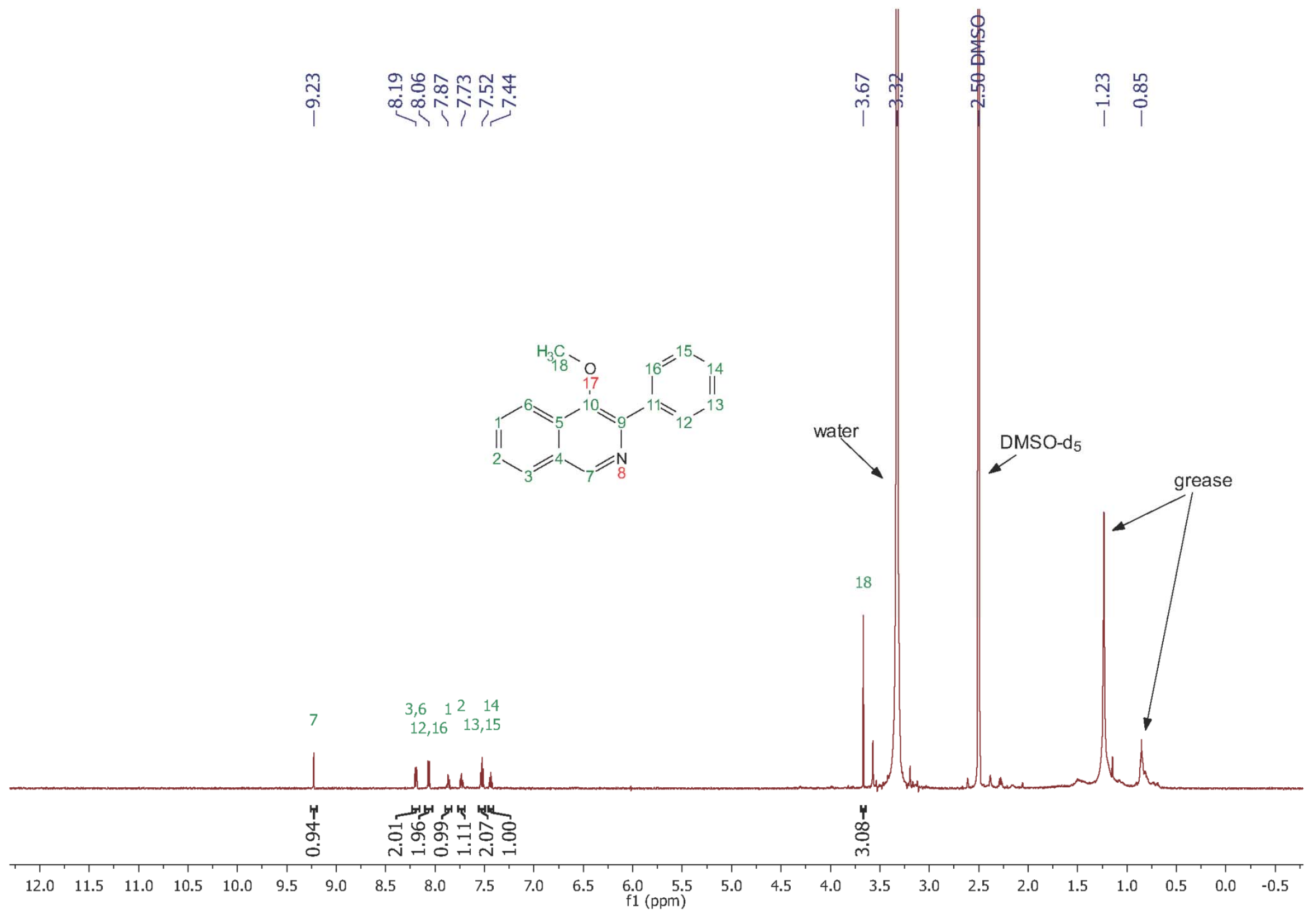


${ }^{1} \mathrm{H} /{ }^{1} \mathrm{H}$ COSY NMR spectrum of $10 \mathrm{~b}\left(600 / 600 \mathrm{MHz}\right.$, DMSO- $\left.\mathrm{d}_{6}\right)$

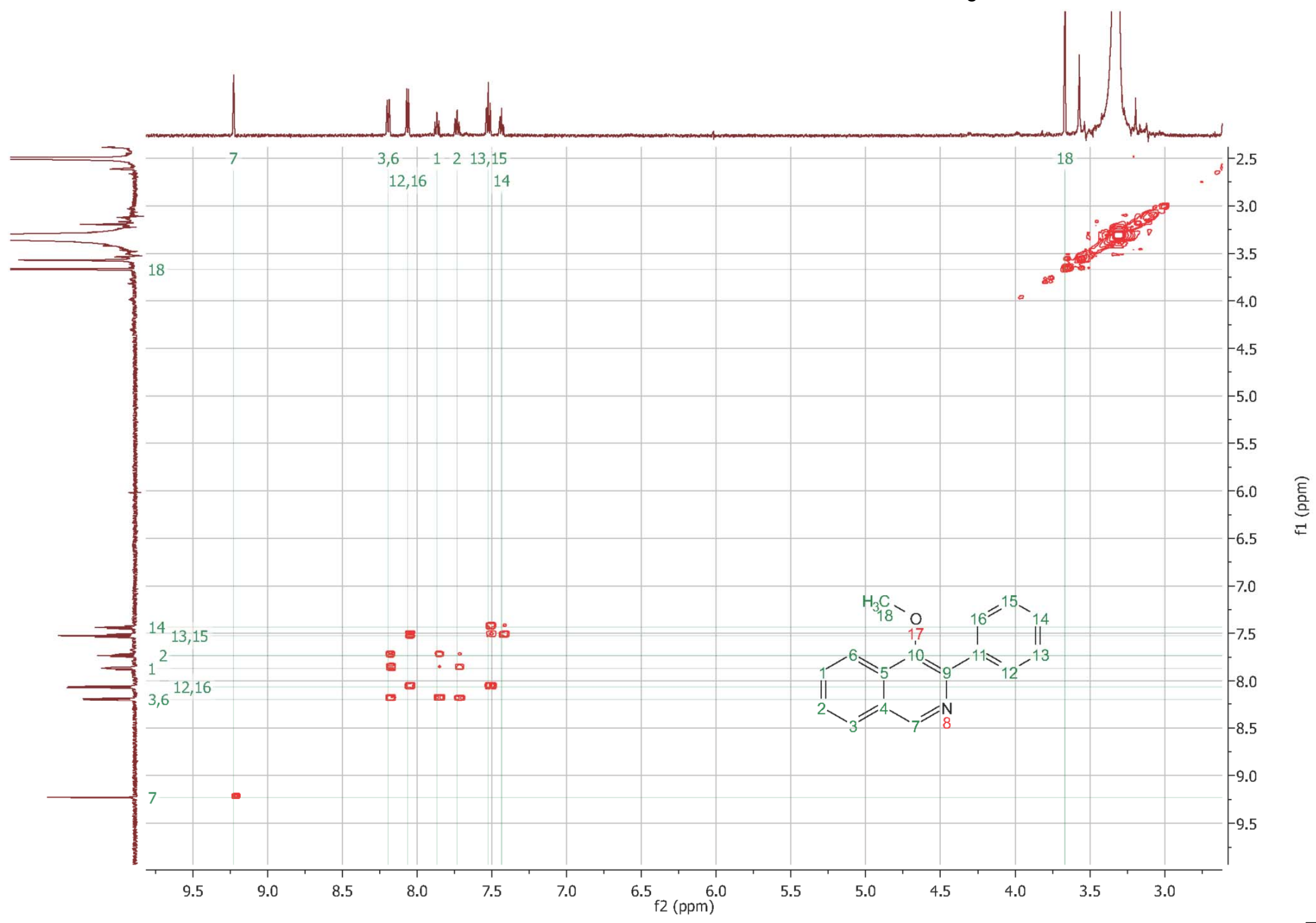


${ }^{1} \mathrm{H} /{ }^{13} \mathrm{C} \mathrm{HSQC} \mathrm{NMR} \mathrm{spectrum} \mathrm{of} \mathrm{10b}\left(600 / 150 \mathrm{MHz}\right.$, DMSO-d $\left.{ }_{6}\right)$

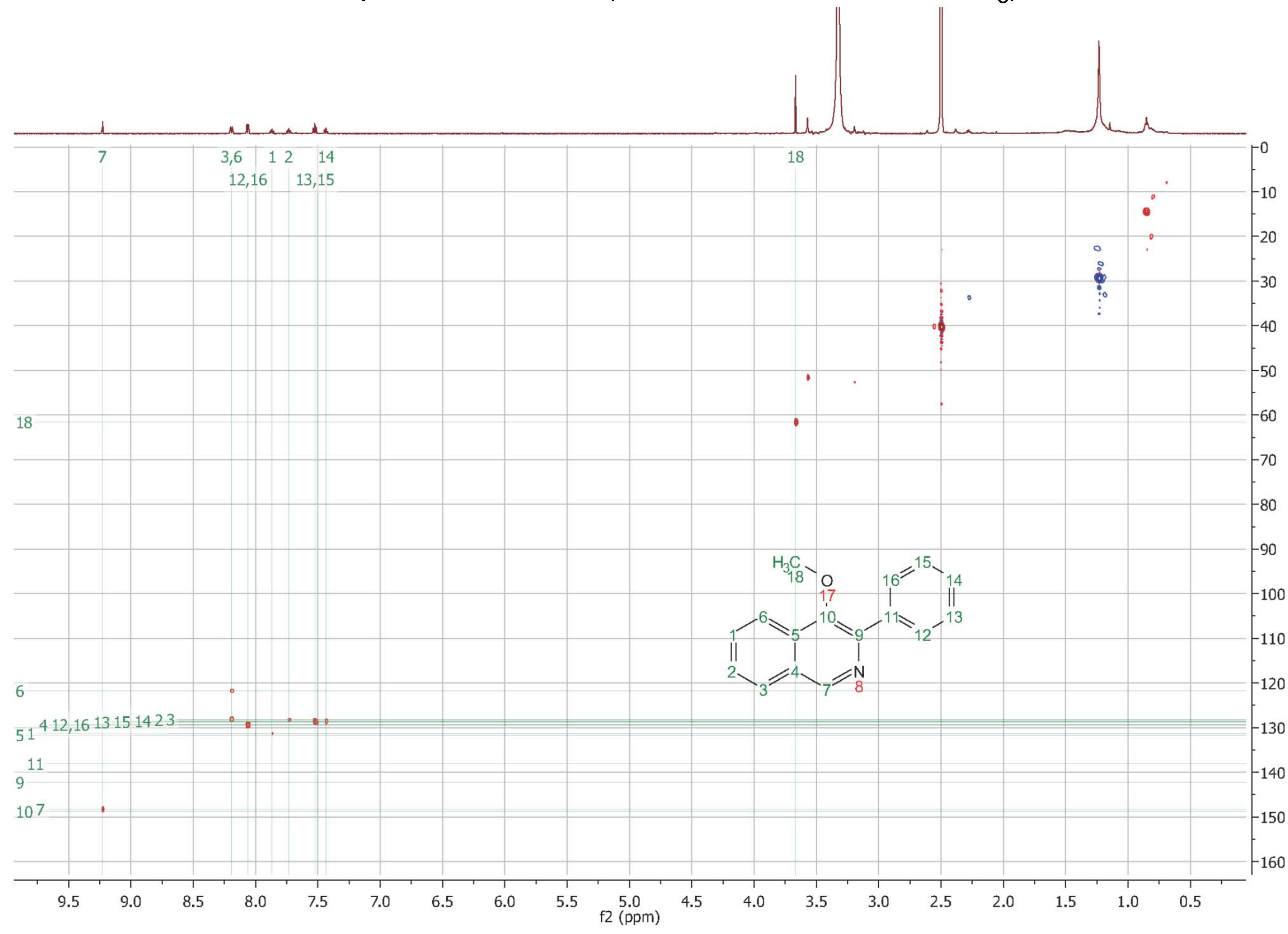


${ }^{1} \mathrm{H} /{ }^{13} \mathrm{C}$ HMBC NMR spectrum of 10b $\left(600 / 150 \mathrm{MHz}\right.$, DMSO-d $\left.\mathrm{d}_{6}\right)$

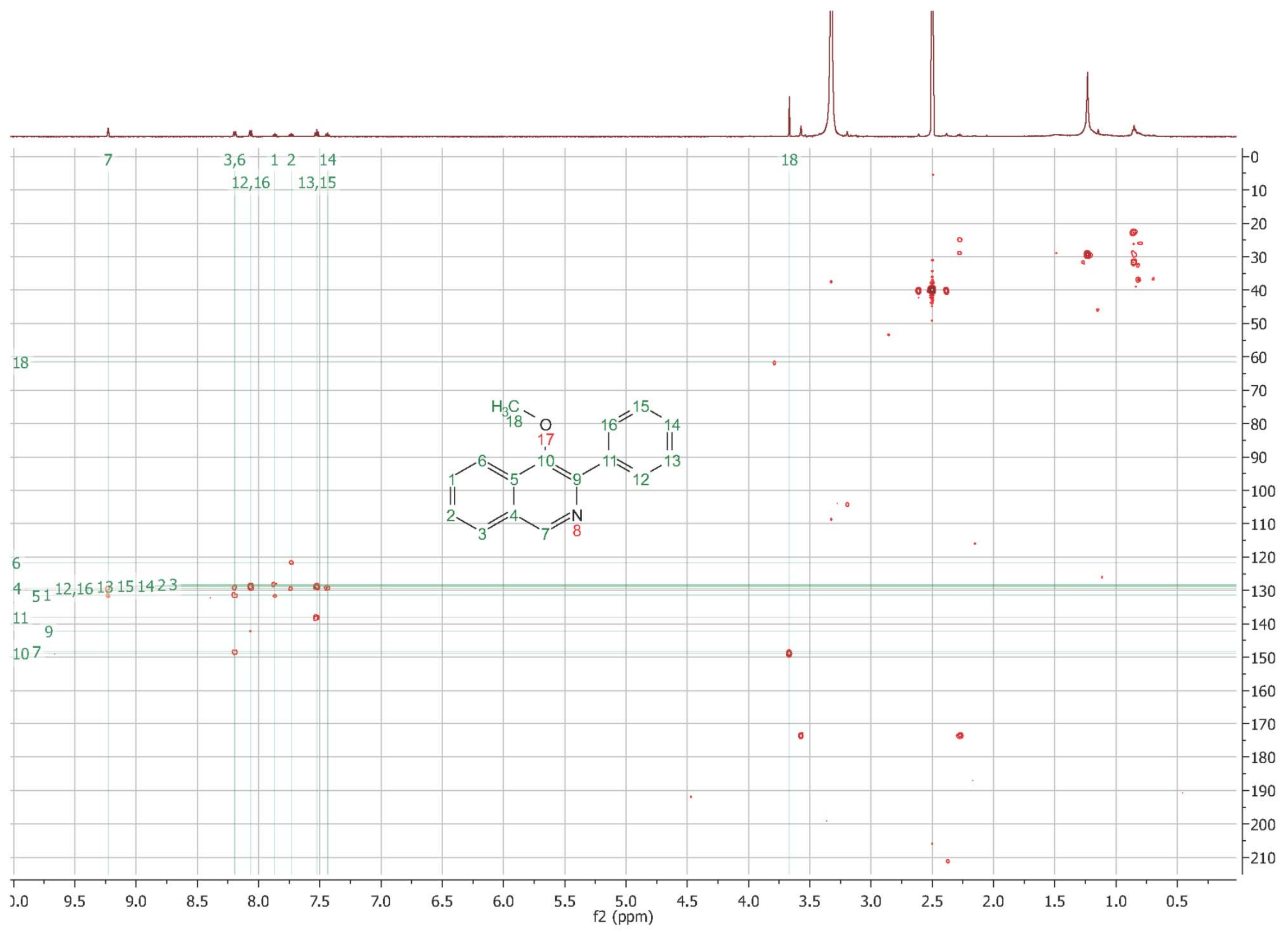


${ }^{1} \mathrm{H}$ NMR spectrum of $10 \mathrm{c}\left(600 \mathrm{MHz}\right.$, DMSO-d $\left.\mathrm{d}_{6}\right)$

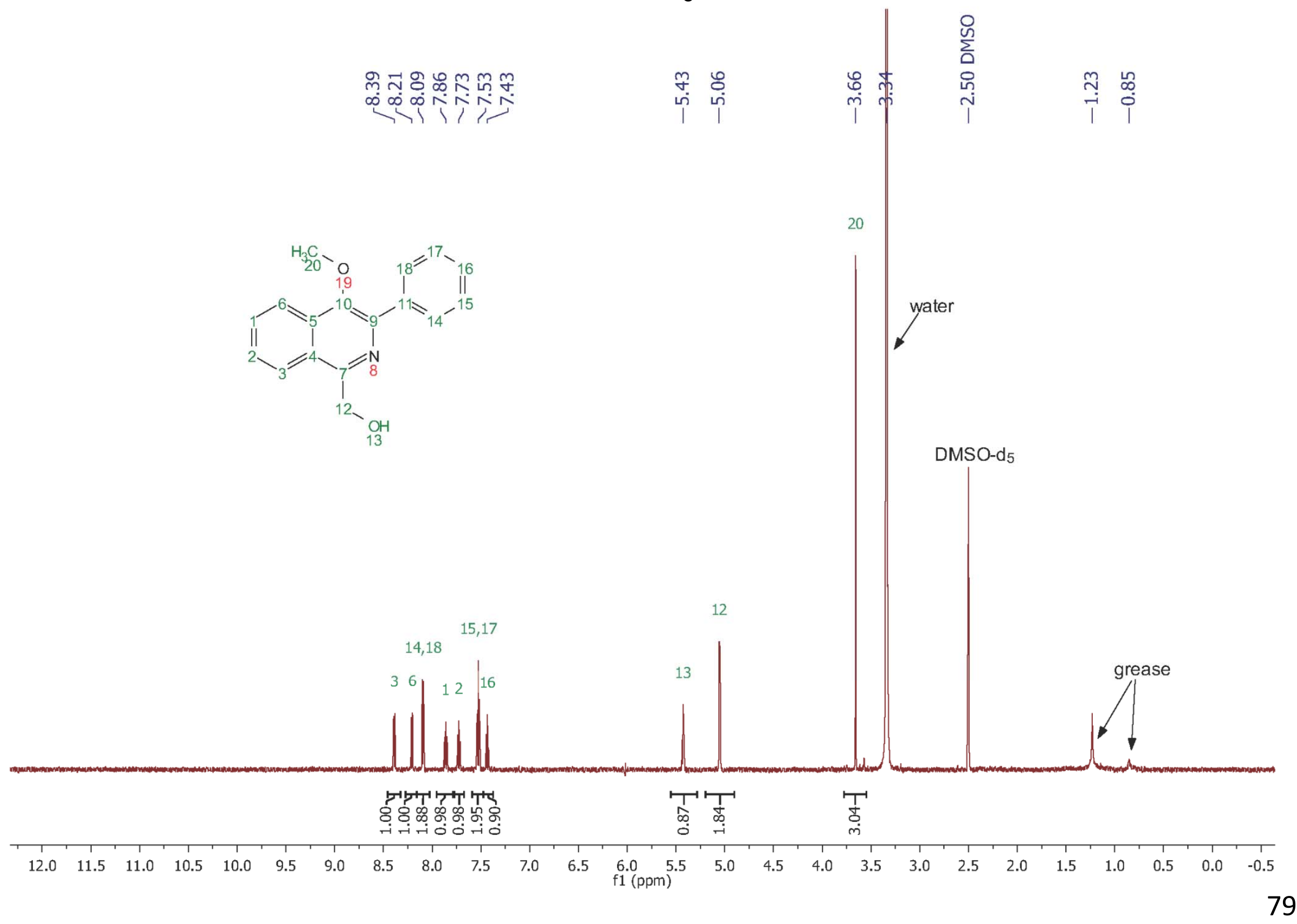


${ }^{13} \mathrm{C}$ NMR spectrum of $10 \mathrm{c}\left(150 \mathrm{MHz}, \mathrm{DMSO}-\mathrm{d}_{6}\right)$
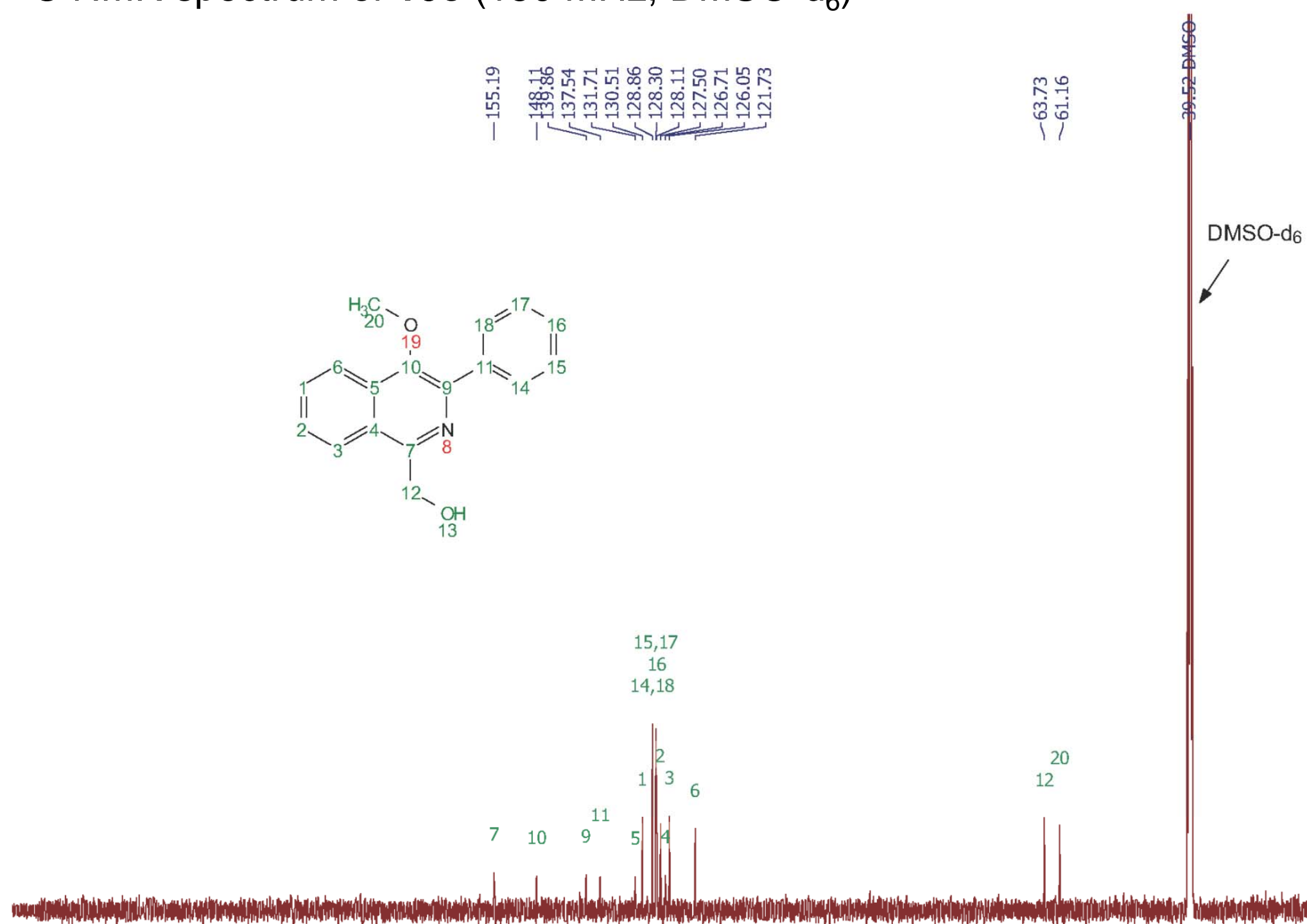
${ }^{1} \mathrm{H} /{ }^{1} \mathrm{H}$ COSY NMR spectrum of $10 \mathrm{c}\left(600 / 600 \mathrm{MHz}\right.$, DMSO- $\left.\mathrm{d}_{6}\right)$

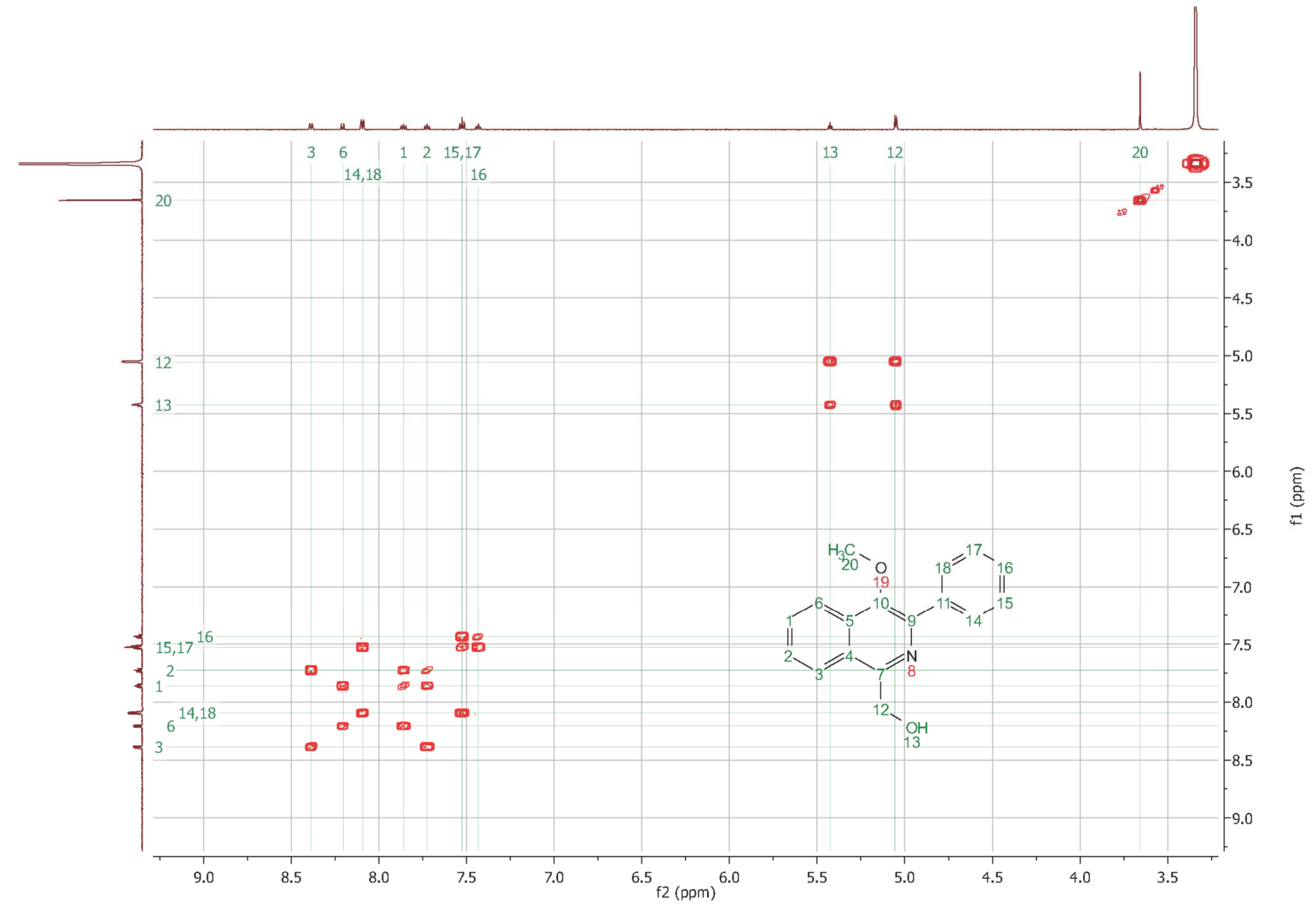


${ }^{1} \mathrm{H} /{ }^{13} \mathrm{C}$ HSQC NMR spectrum of $10 \mathrm{c}\left(600 / 150 \mathrm{MHz}\right.$, DMSO-d $\left.\mathrm{d}_{6}\right)$

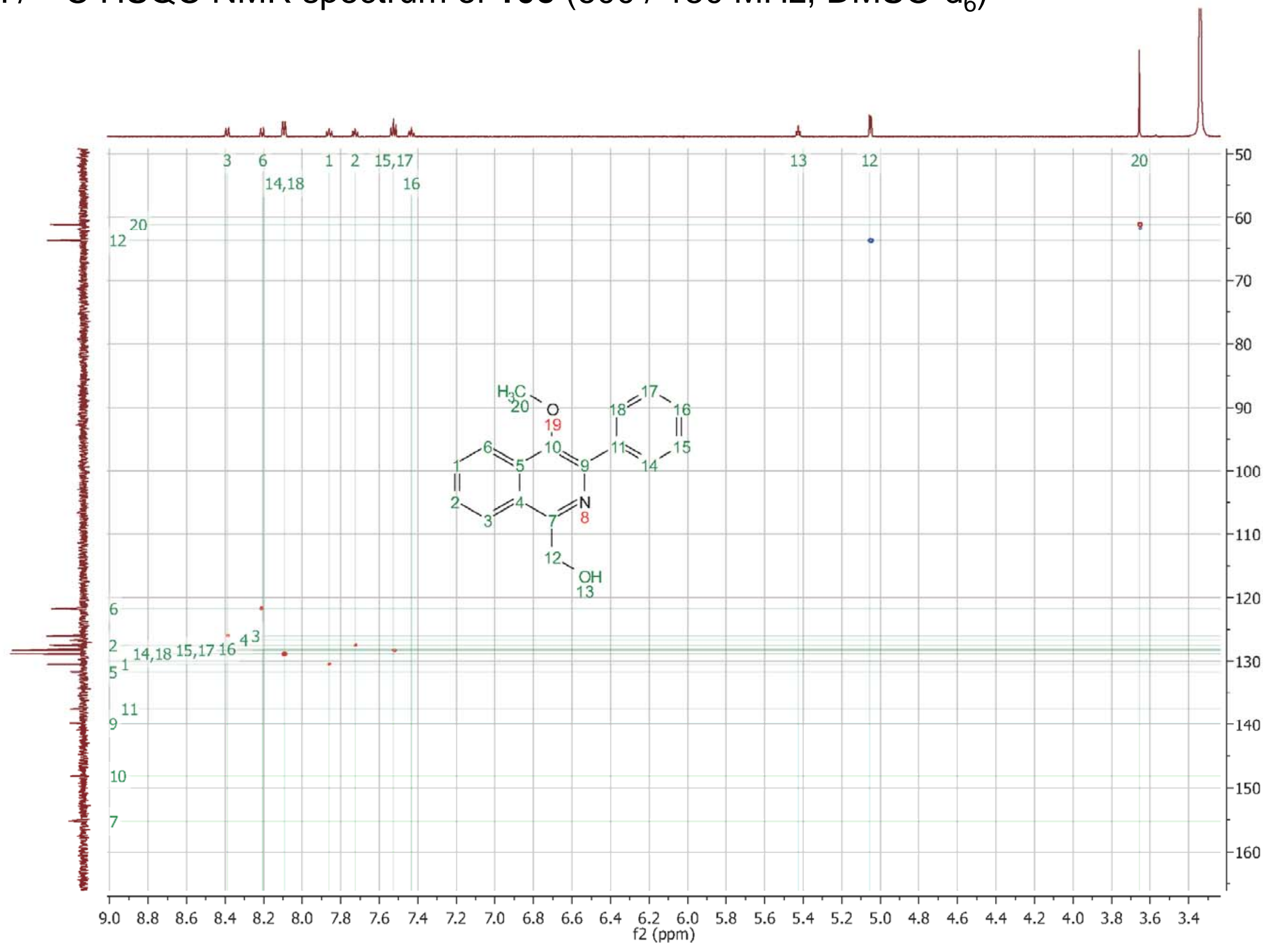


${ }^{1} \mathrm{H} /{ }^{13} \mathrm{C}$ HMBC NMR spectrum of $10 \mathrm{c}\left(600 / 150 \mathrm{MHz}\right.$, DMSO-d $\left.\mathrm{d}_{6}\right)$

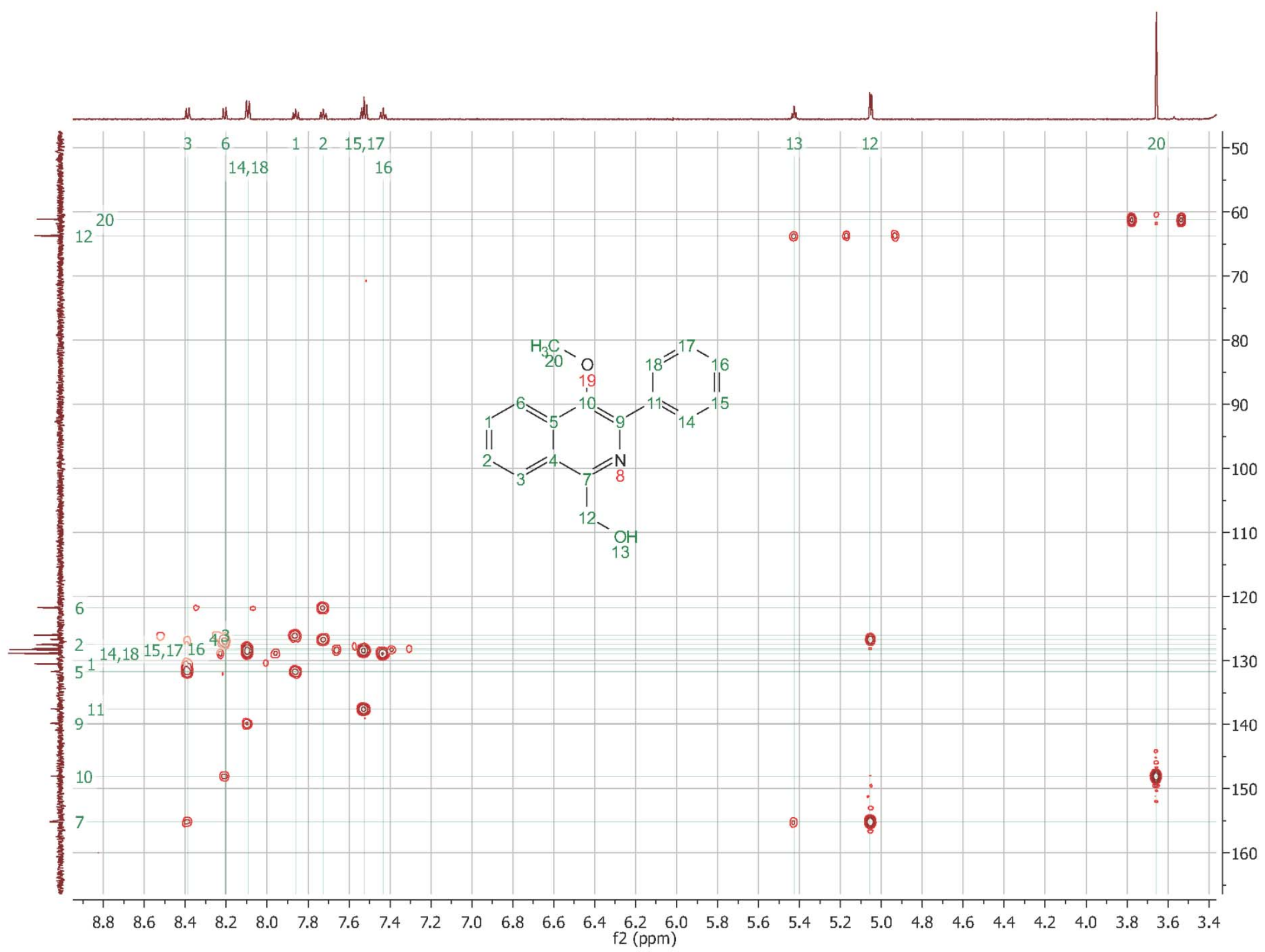


${ }^{1} \mathrm{H} /{ }^{1} \mathrm{H}$ ROESY NMR spectrum of $10 \mathrm{c}\left(600 / 600 \mathrm{MHz}\right.$, DMSO-d $\left.\mathrm{d}_{6}\right)$

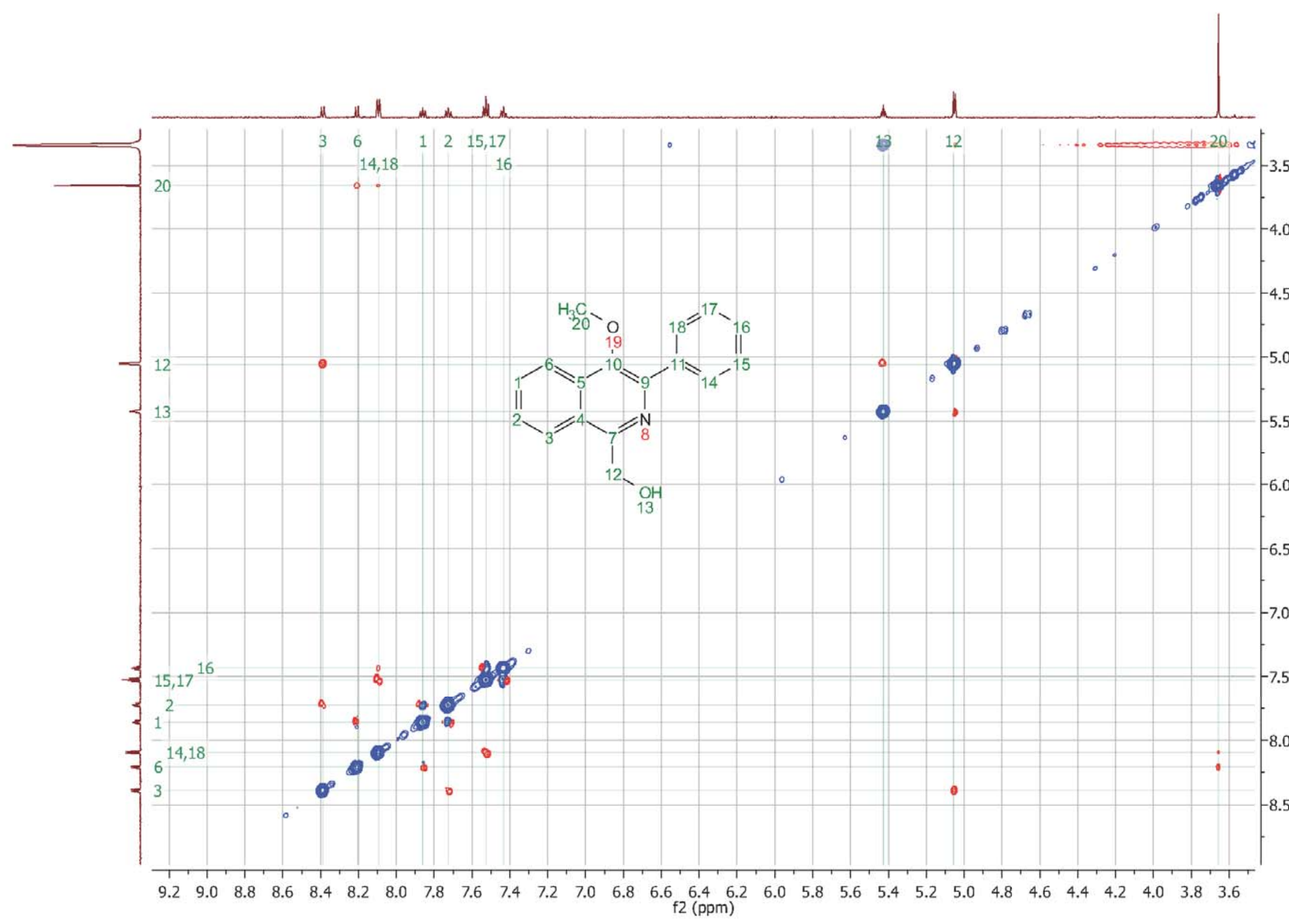


${ }^{1} \mathrm{H}$ NMR spectrum of $11 \mathrm{a}\left(600 \mathrm{MHz}\right.$, DMSO-d $\left.\mathrm{d}_{6}\right)$

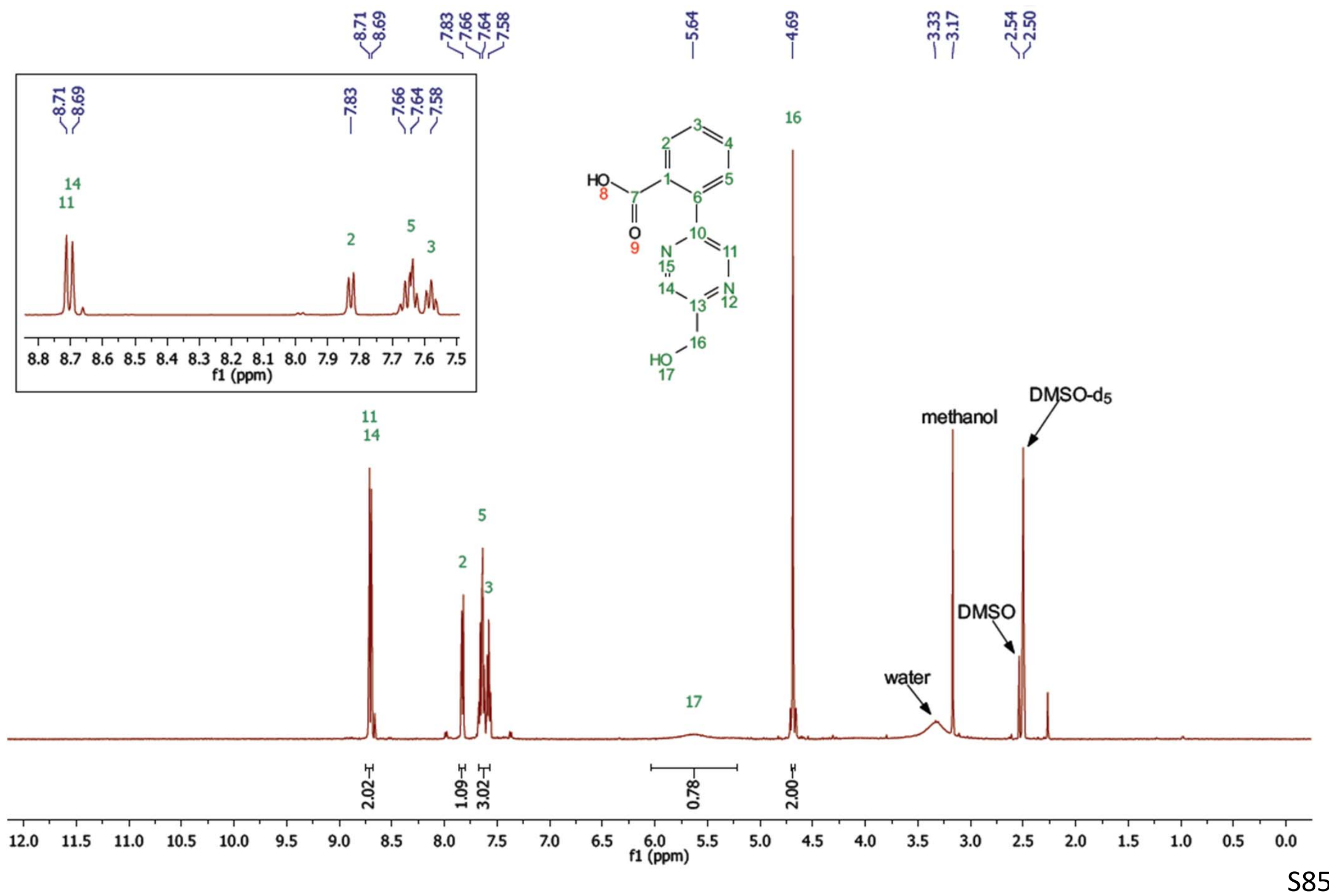


${ }^{13} \mathrm{C}$ NMR spectrum of $11 \mathrm{a}\left(150 \mathrm{MHz}, \mathrm{DMSO}-\mathrm{d}_{6}\right)$

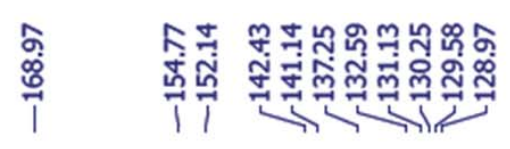

กั่
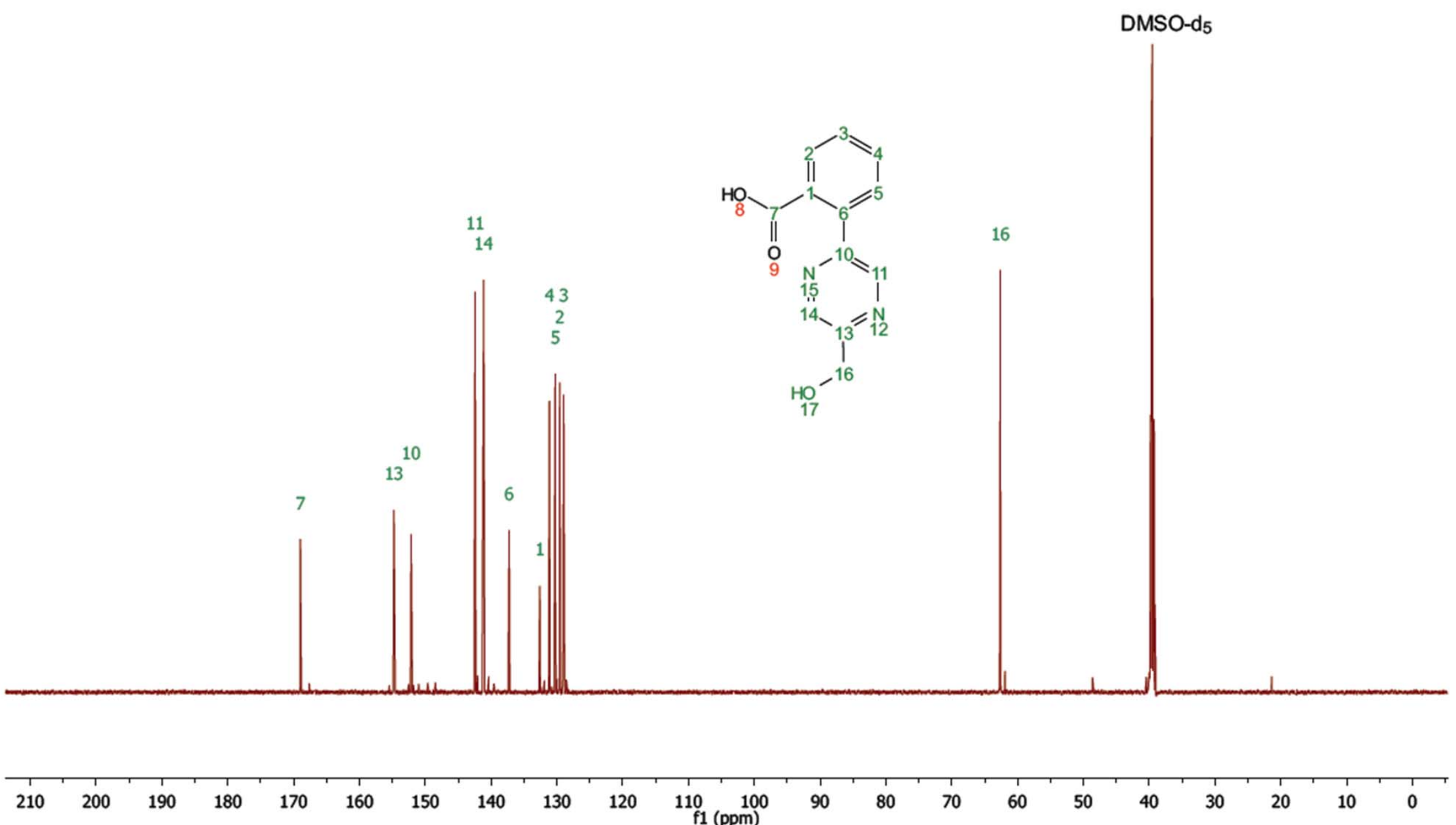
${ }^{1} \mathrm{H} /{ }^{1} \mathrm{H}$ COSY NMR spectrum of $11 \mathrm{a}\left(600 / 600 \mathrm{MHz}\right.$, DMSO- $\left.\mathrm{d}_{6}\right)$

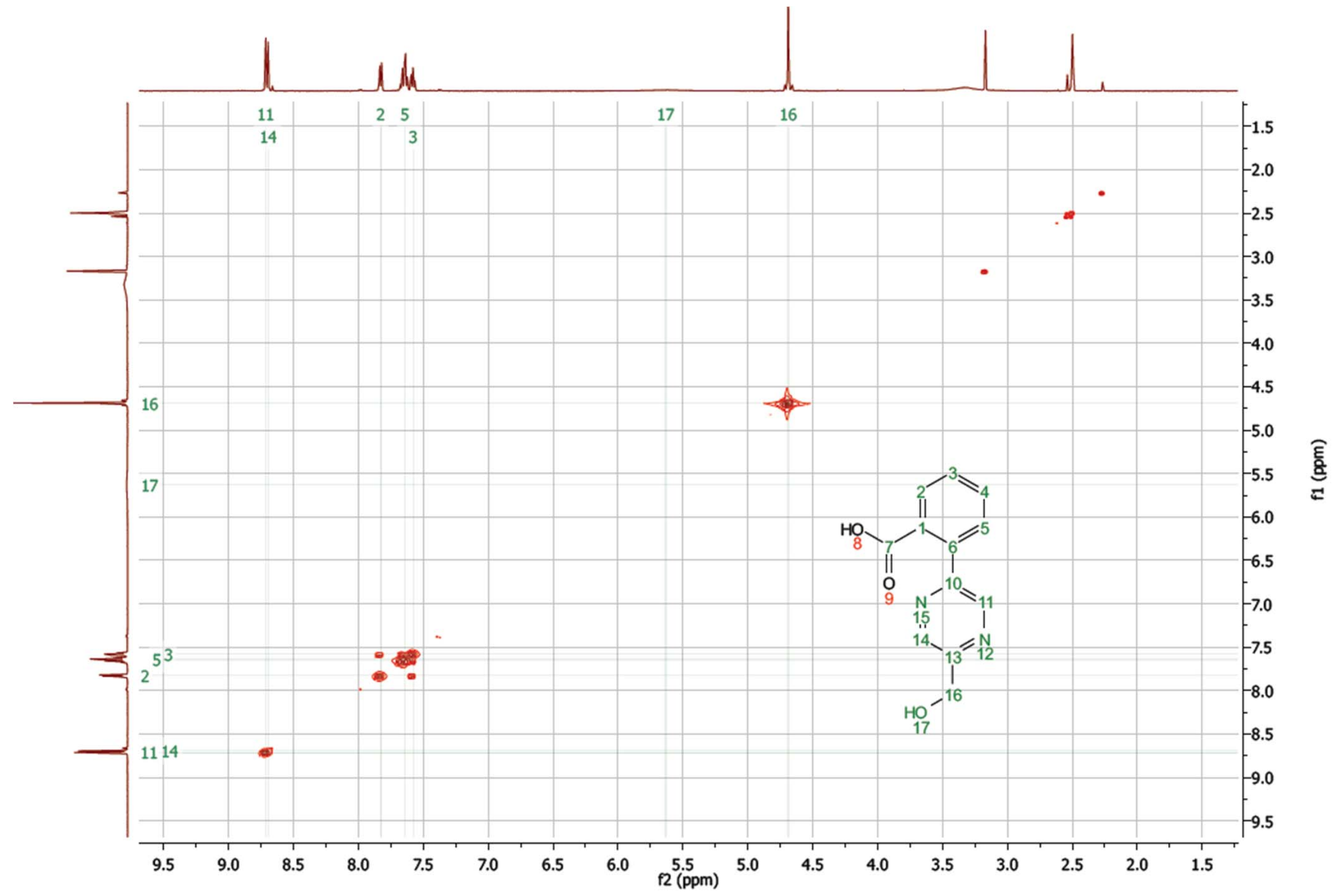


${ }^{1} \mathrm{H} /{ }^{13} \mathrm{C}$ HSQC NMR spectrum of $\mathbf{1 1 a}\left(600 / 150 \mathrm{MHz}\right.$, DMSO-d $\left.{ }_{6}\right)$

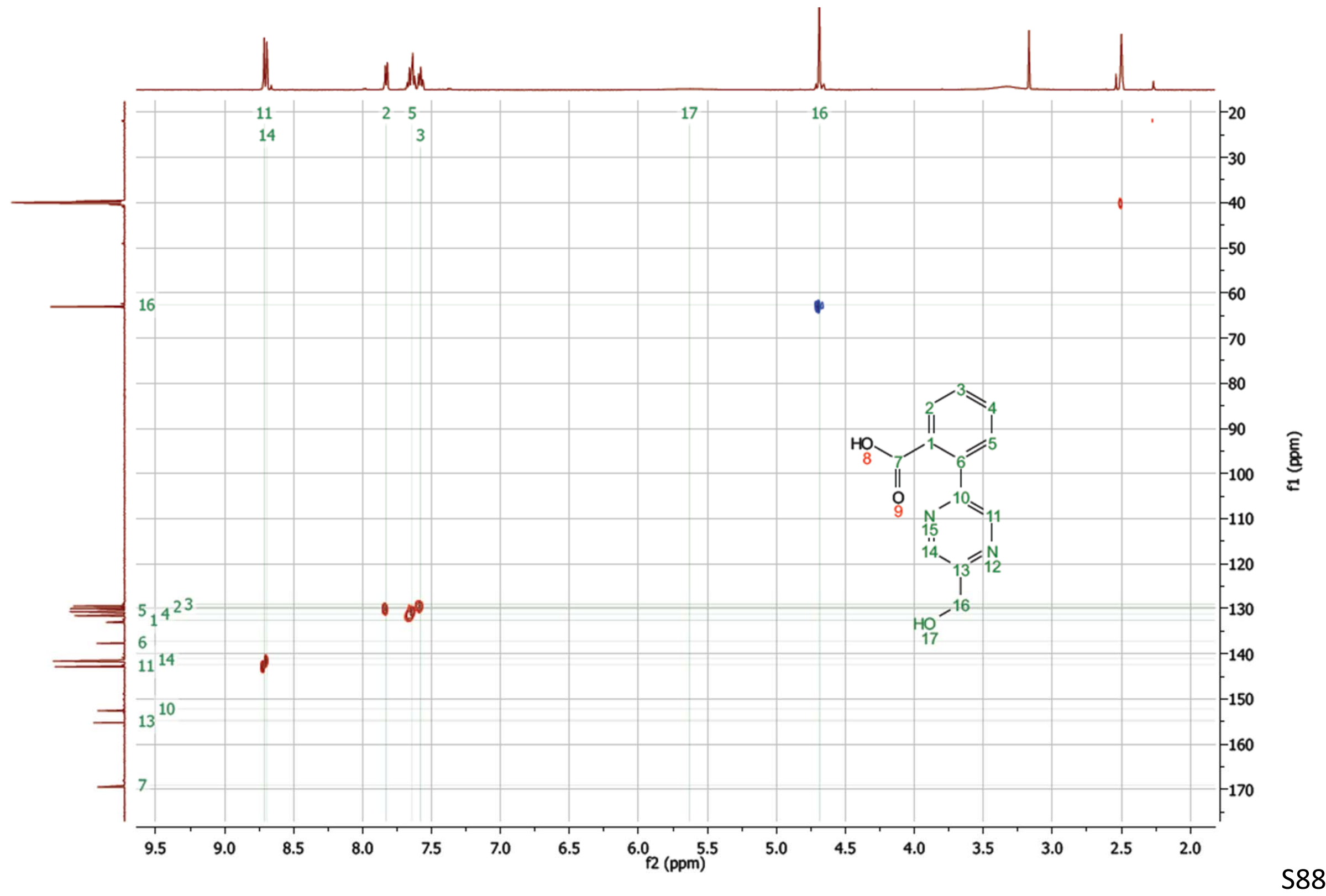


${ }^{1} \mathrm{H} /{ }^{13} \mathrm{C}$ HMBC NMR spectrum of $11 \mathrm{a}\left(600 / 150 \mathrm{MHz}\right.$, DMSO-d $\left.\mathrm{d}_{6}\right)$

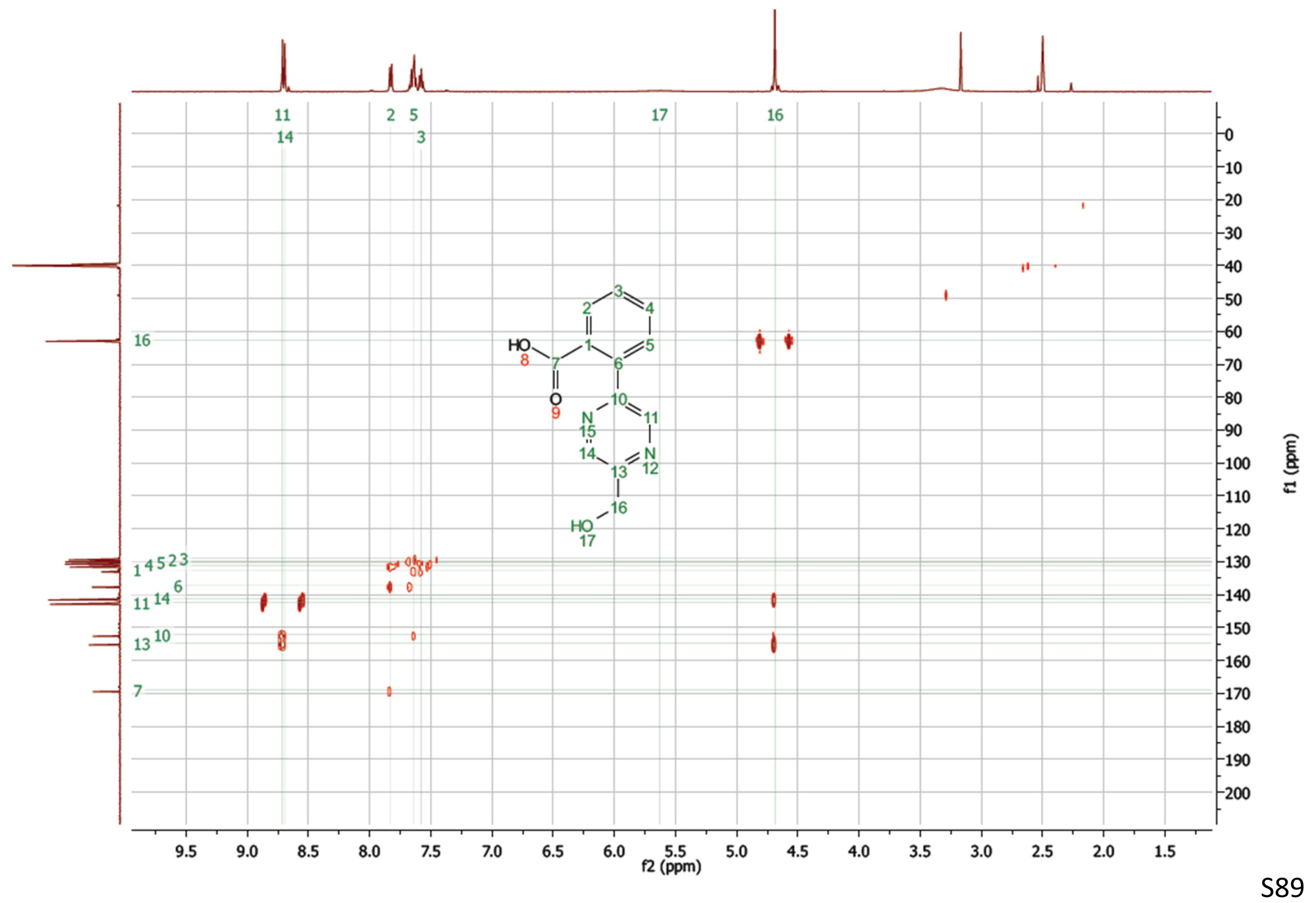


${ }^{1} \mathrm{H} /{ }^{1} \mathrm{H}$ ROESY NMR spectrum of $11 \mathrm{a}\left(600 / 600 \mathrm{MHz}\right.$, DMSO-d $\left.\mathrm{d}_{6}\right)$

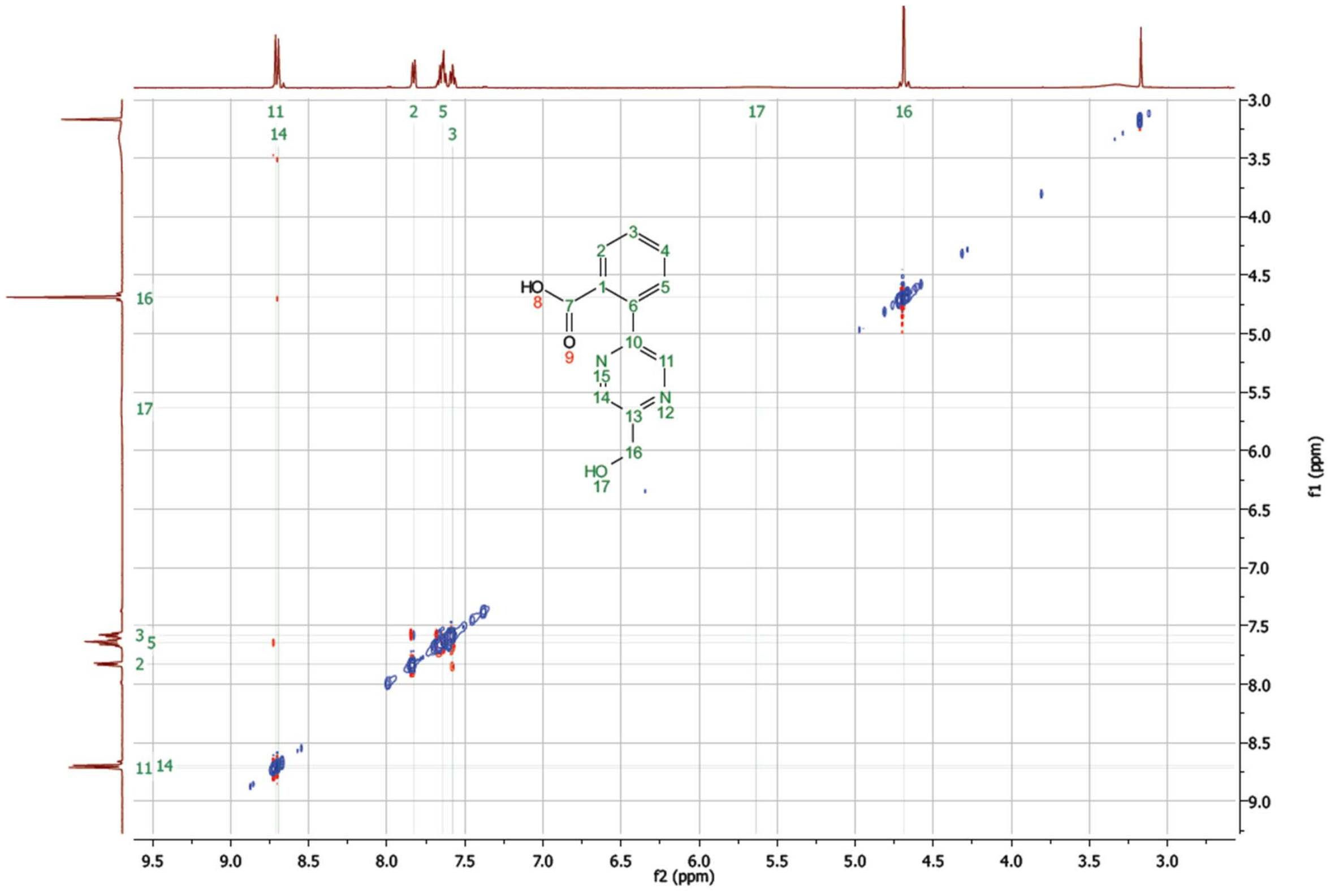


${ }^{1} \mathrm{H} /{ }^{15} \mathrm{~N} \mathrm{HMBC}$ NMR spectrum of $11 \mathrm{a}\left(500 / 51 \mathrm{MHz}, \mathrm{DMSO}-\mathrm{d}_{6}\right)$

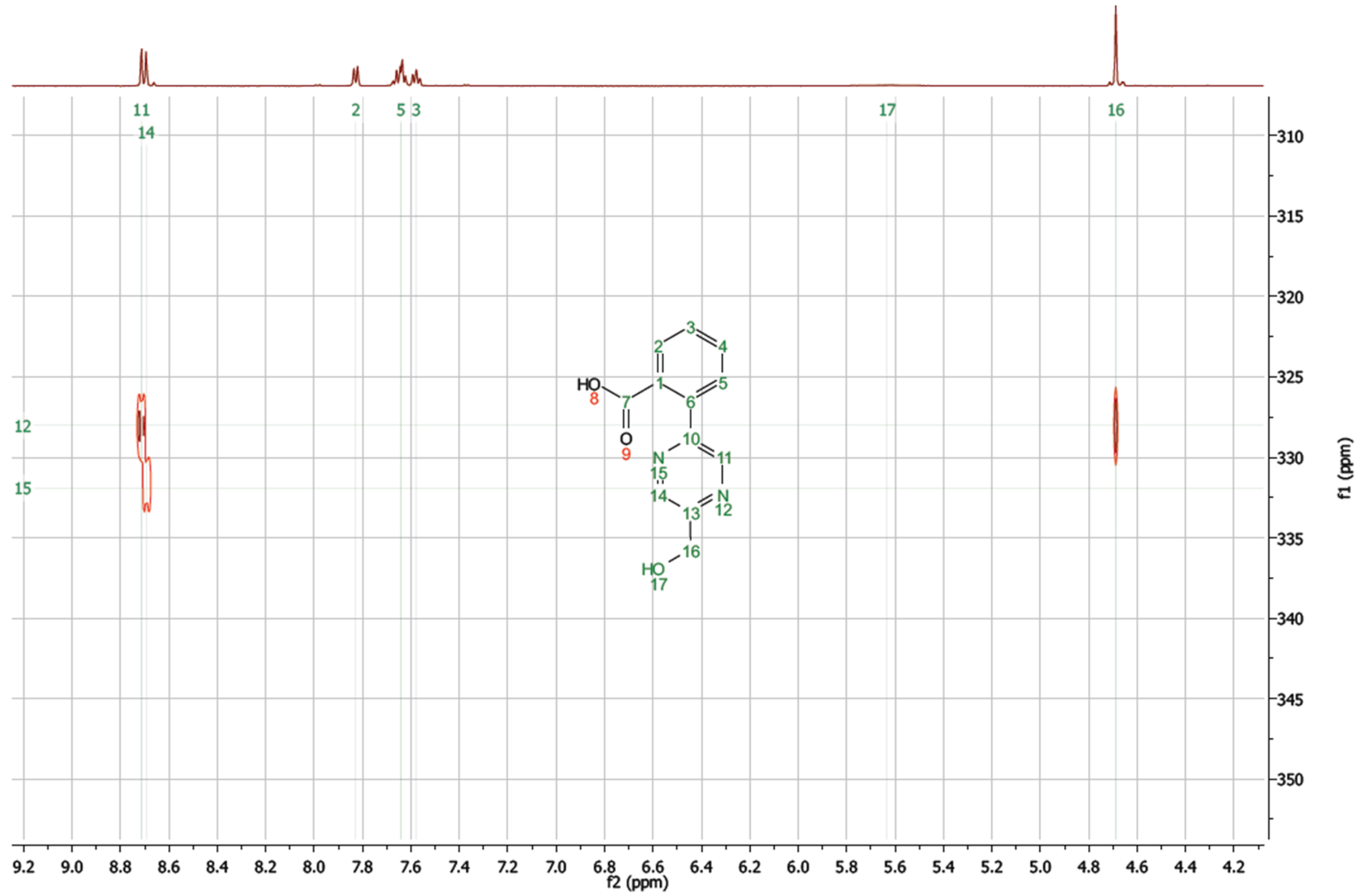




\section{${ }^{1} \mathrm{H}$ selective 1D NOE NMR spectrum of $11 \mathrm{a}\left(600 \mathrm{MHz}, \mathrm{DMSO}-\mathrm{d}_{6}\right)$}

irradiate proton \#16, then observe strong NOE to proton \#14 and weak NOE to proton\#11
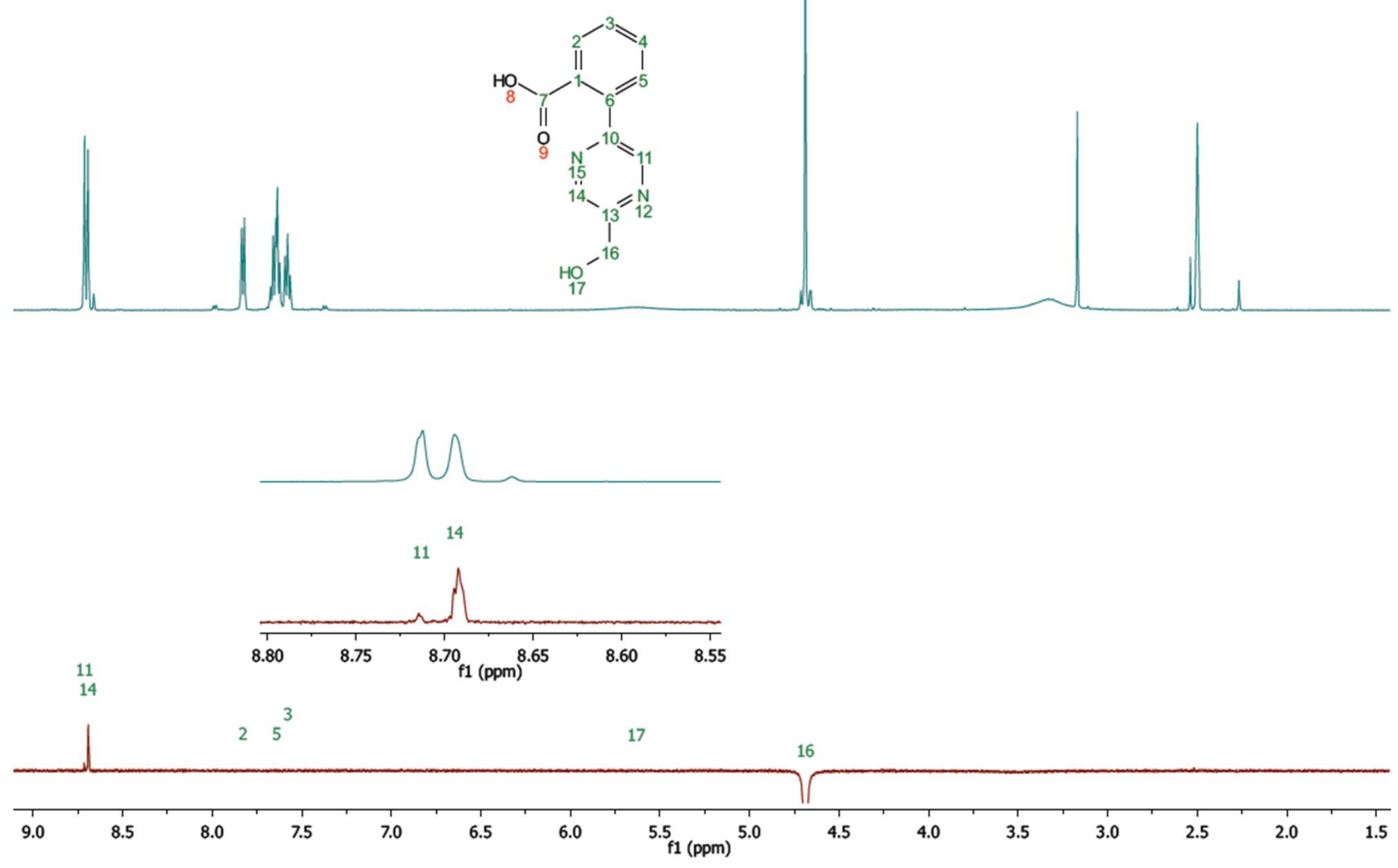
${ }^{1} \mathrm{H}$ NMR spectrum of $\mathbf{1 1 b}\left(600 \mathrm{MHz}\right.$, DMSO- $\left.\mathrm{d}_{6}\right)$

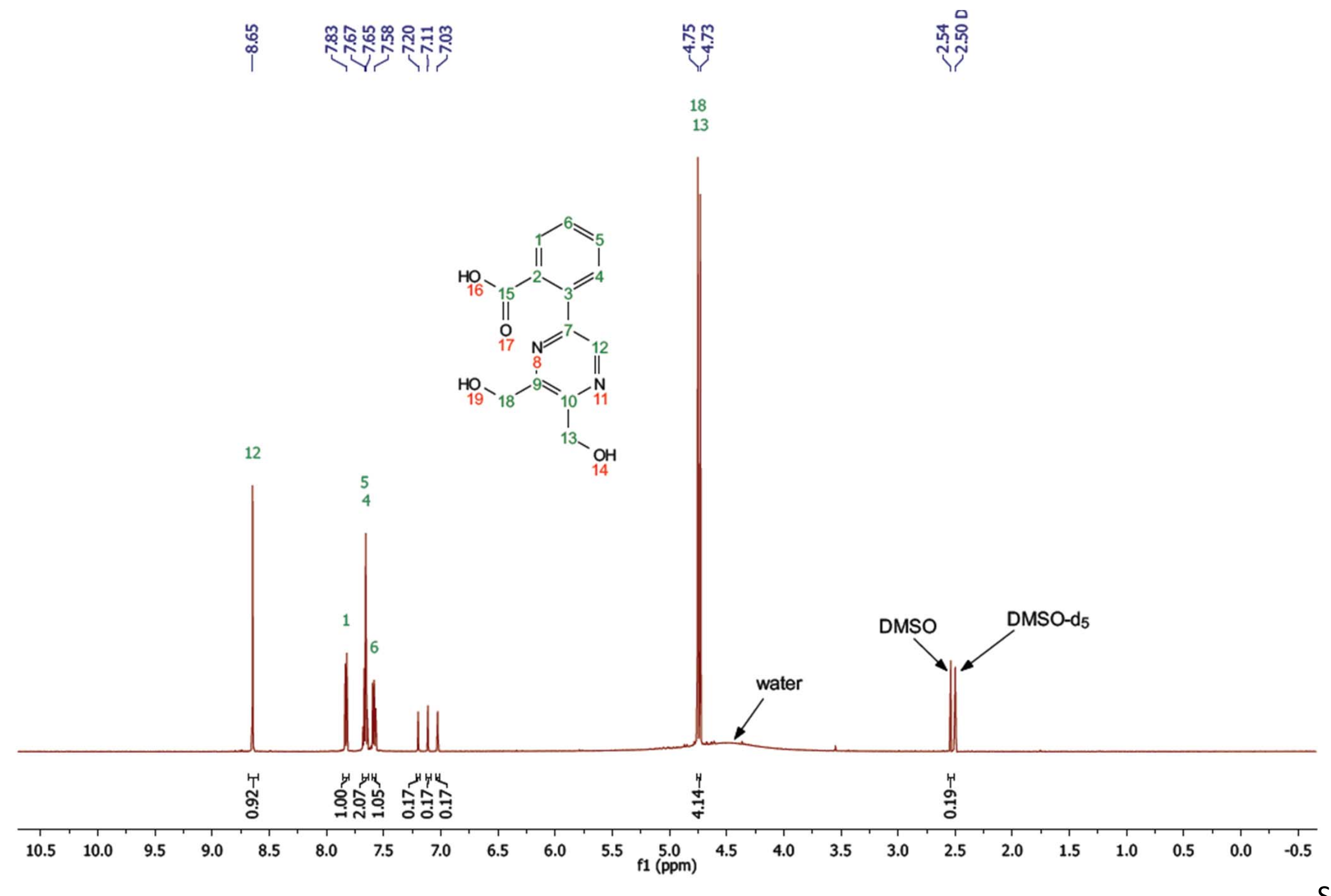


${ }^{13} \mathrm{C}$ NMR spectrum of 11b (150 MHz, DMSO- $\left.\mathrm{d}_{6}\right)$
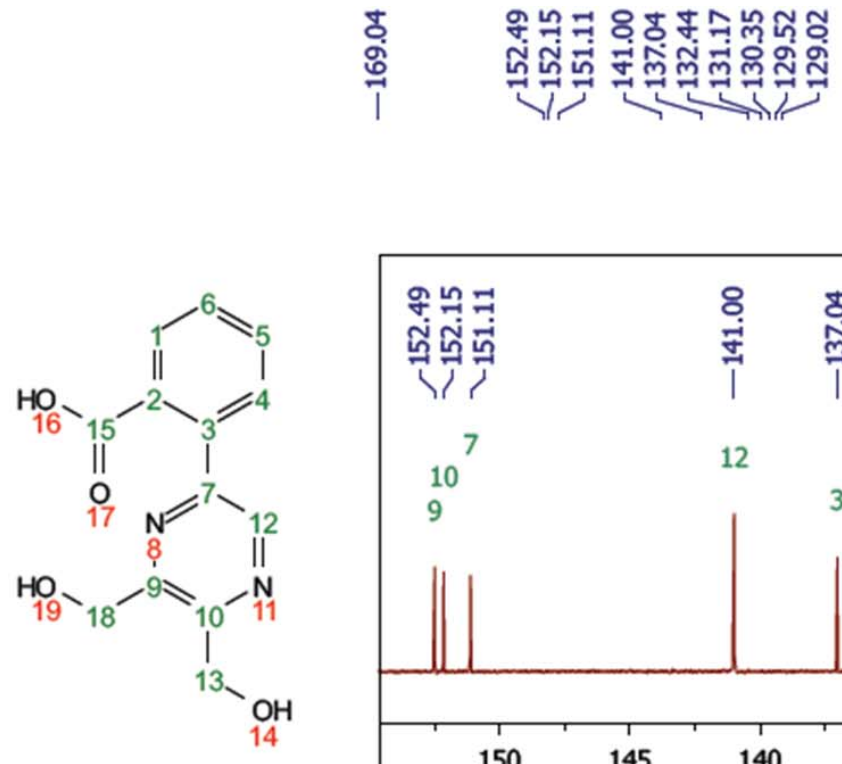
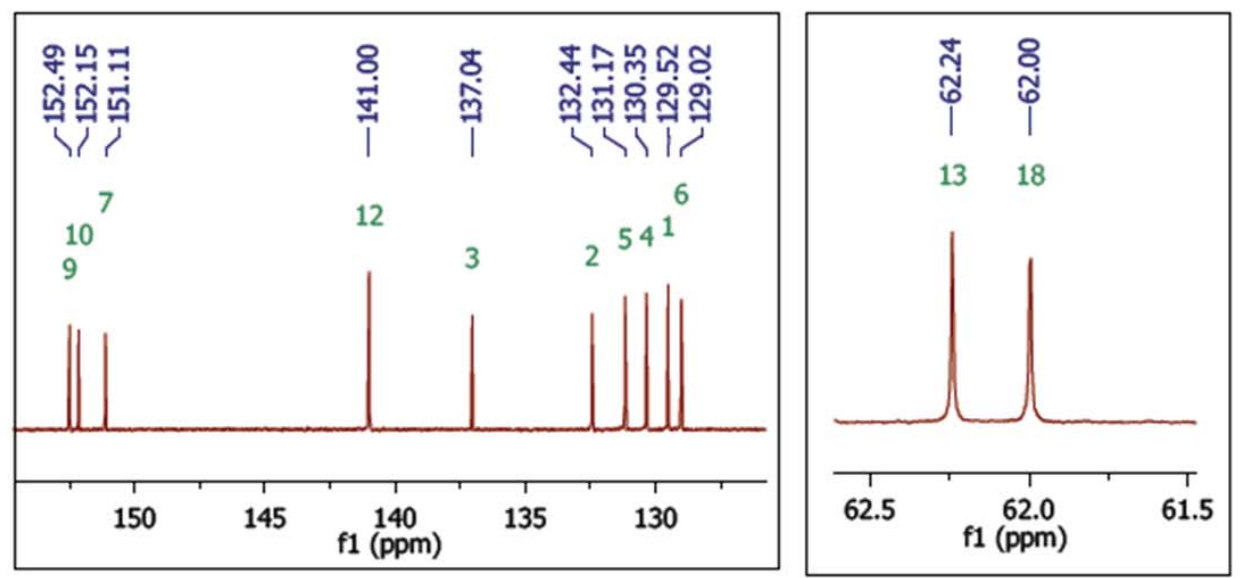

กิ๊

DMSO-d6
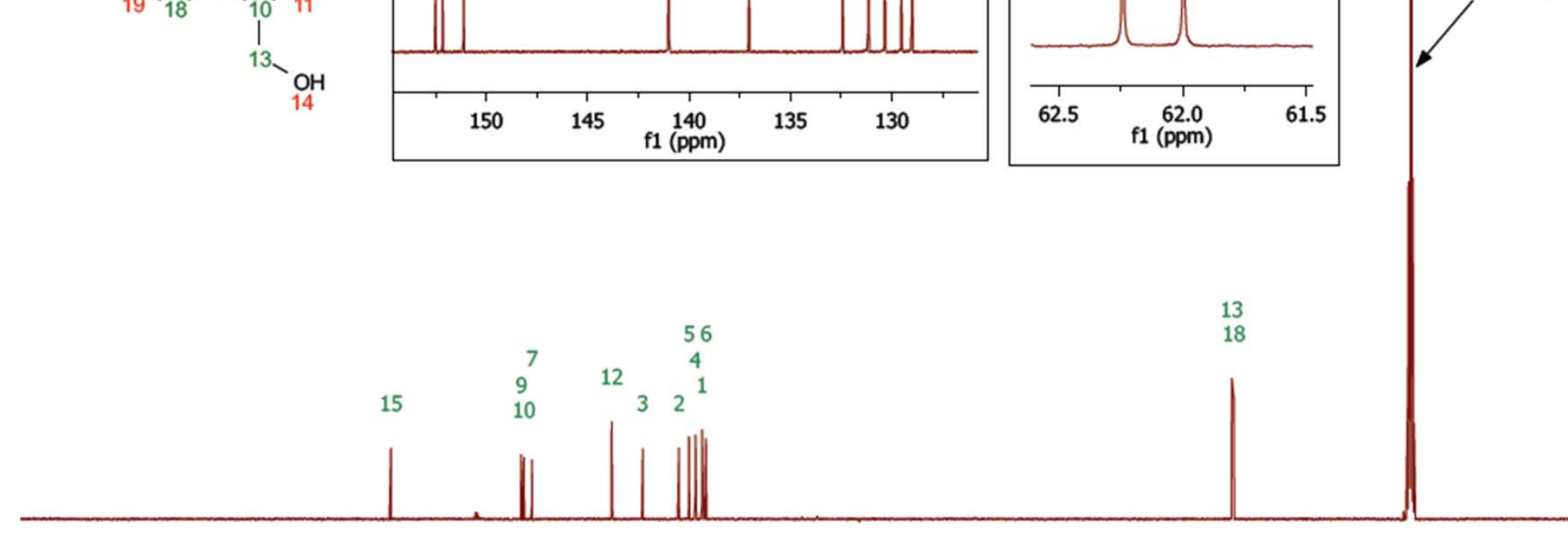
${ }^{1} \mathrm{H} /{ }^{1} \mathrm{H}$ COSY NMR spectrum of $11 b\left(600 / 600 \mathrm{MHz}\right.$, DMSO-d $\left.{ }_{6}\right)$

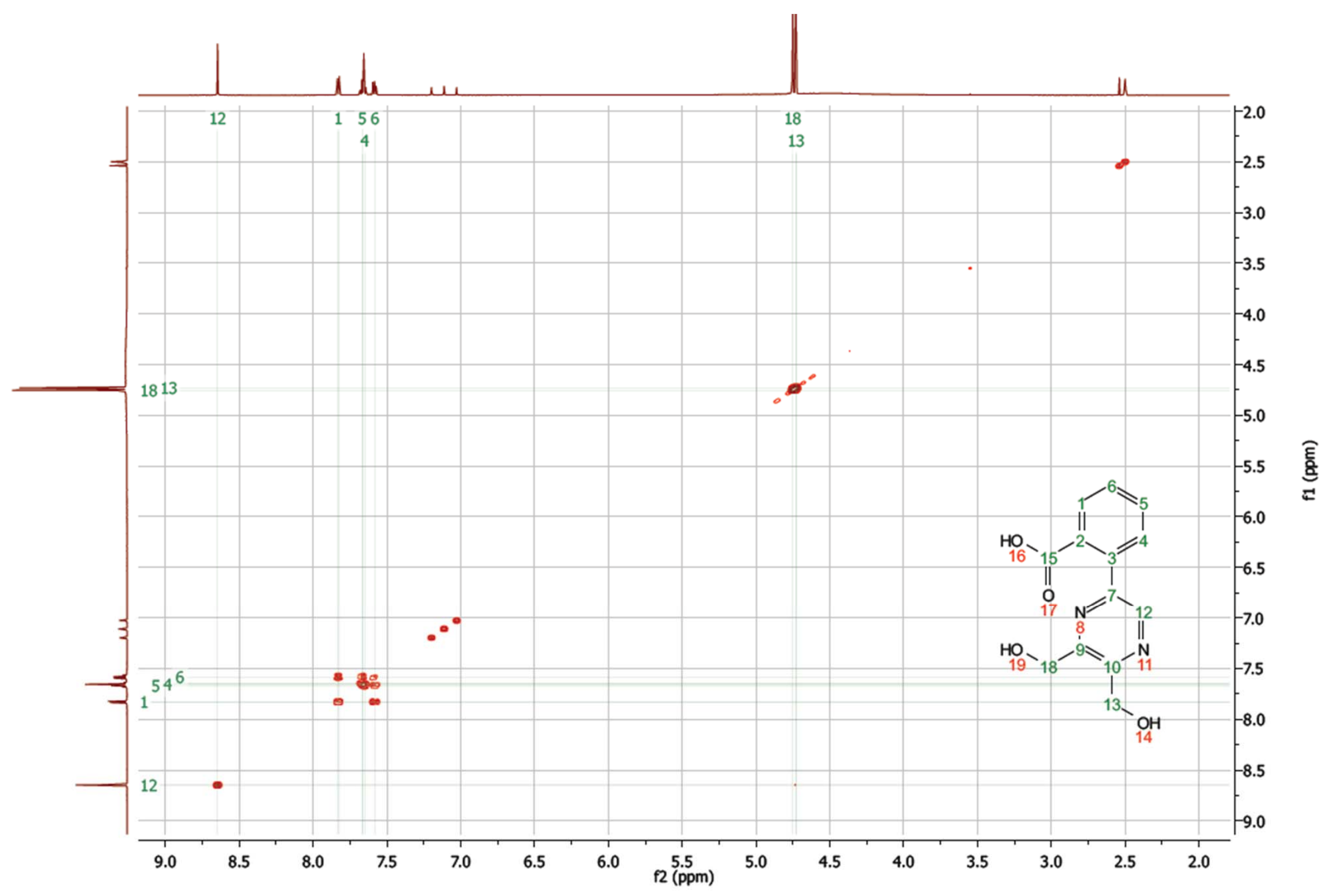


${ }^{1} \mathrm{H} /{ }^{13} \mathrm{C}$ HSQC NMR spectrum of $\mathbf{1 1 b}\left(600 / 150 \mathrm{MHz}\right.$, DMSO-d $\left.\mathrm{d}_{6}\right)$

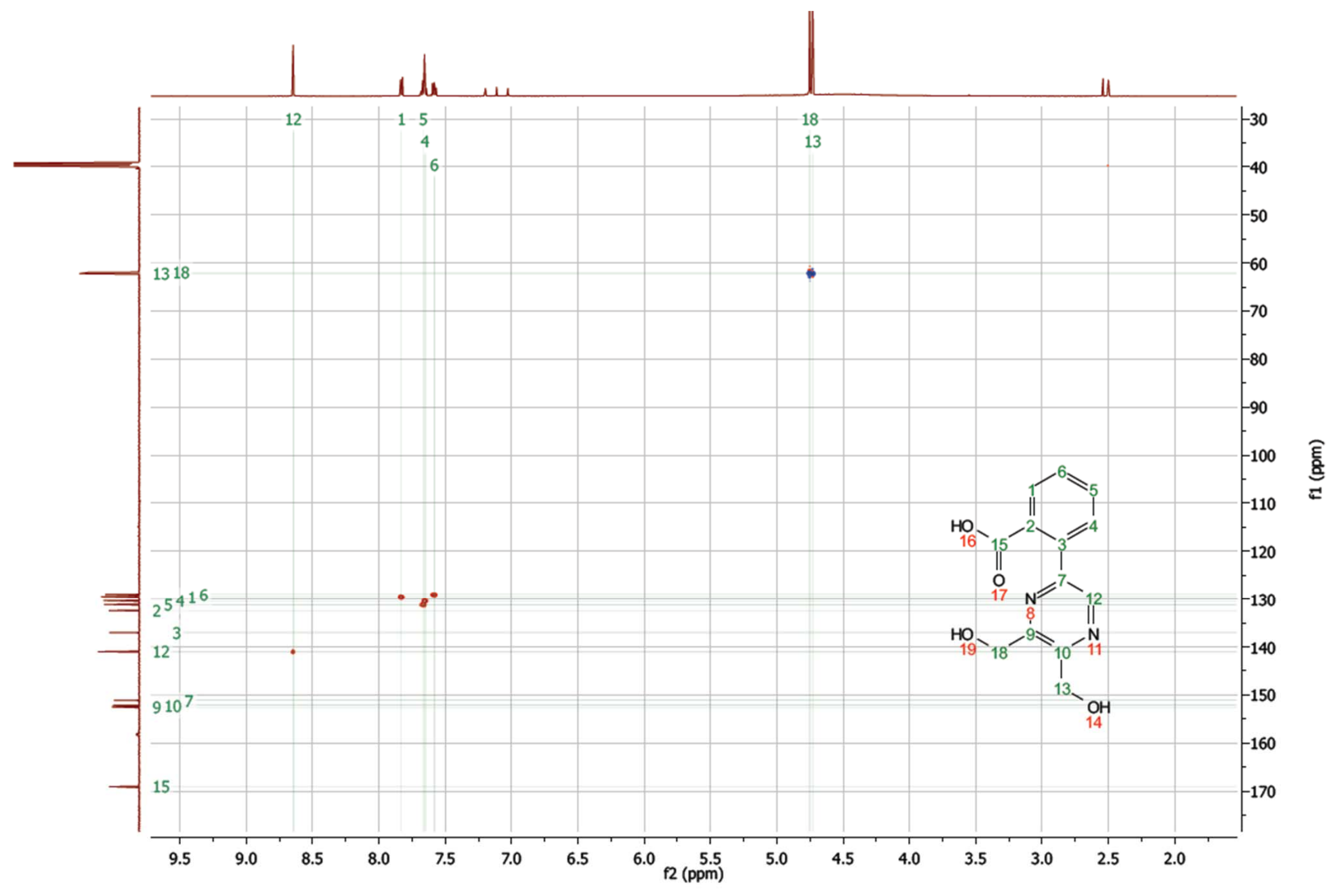


${ }^{1} \mathrm{H} /{ }^{13} \mathrm{C}$ HMBC NMR spectrum of $\mathbf{1 1 b}\left(600 / 150 \mathrm{MHz}\right.$, DMSO-d $\left.\mathrm{d}_{6}\right)$

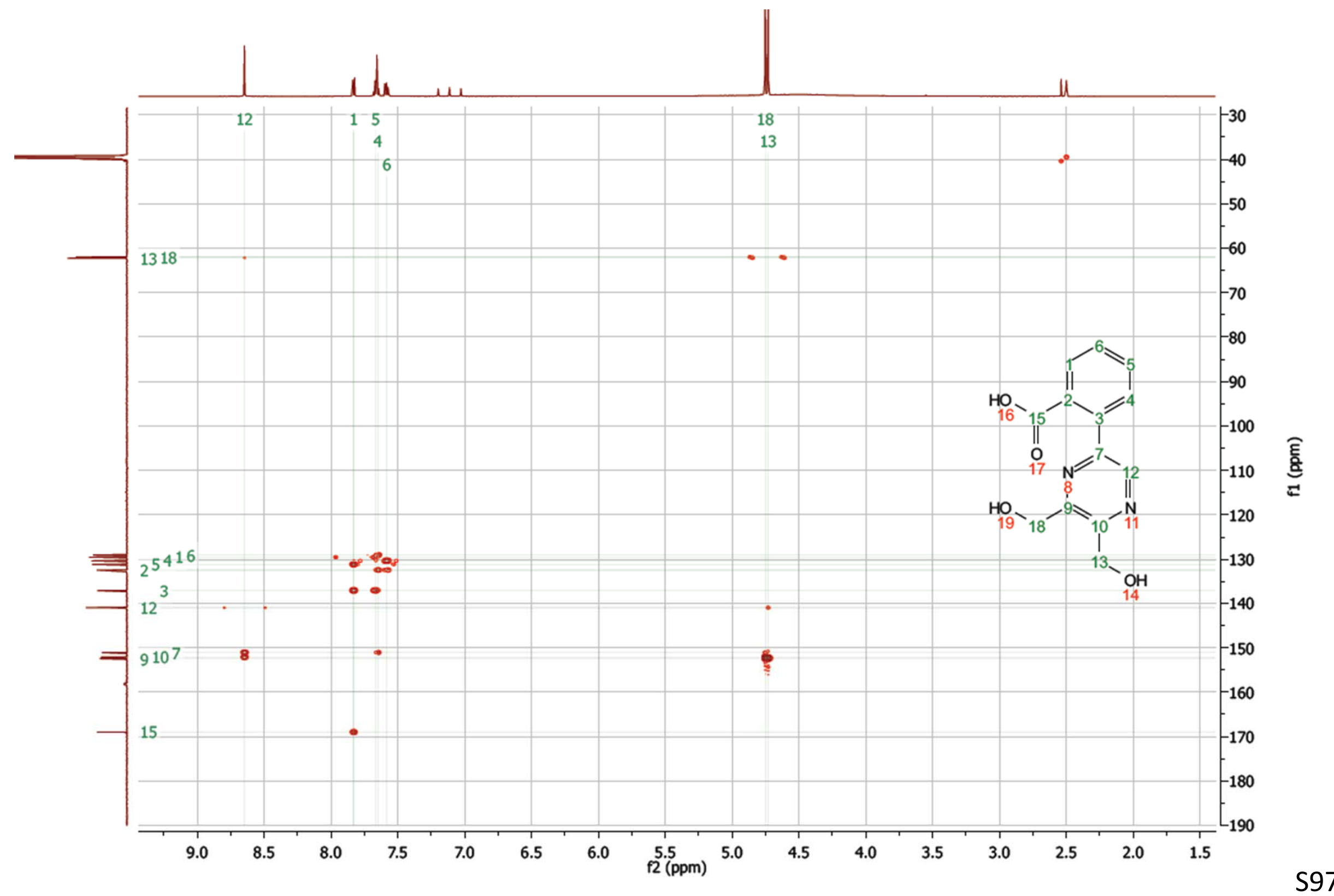


${ }^{1} \mathrm{H} /{ }^{1} \mathrm{H}$ ROESY NMR spectrum of $11 \mathbf{b}\left(600 / 600 \mathrm{MHz}\right.$, DMSO-d $\left.\mathrm{d}_{6}\right)$

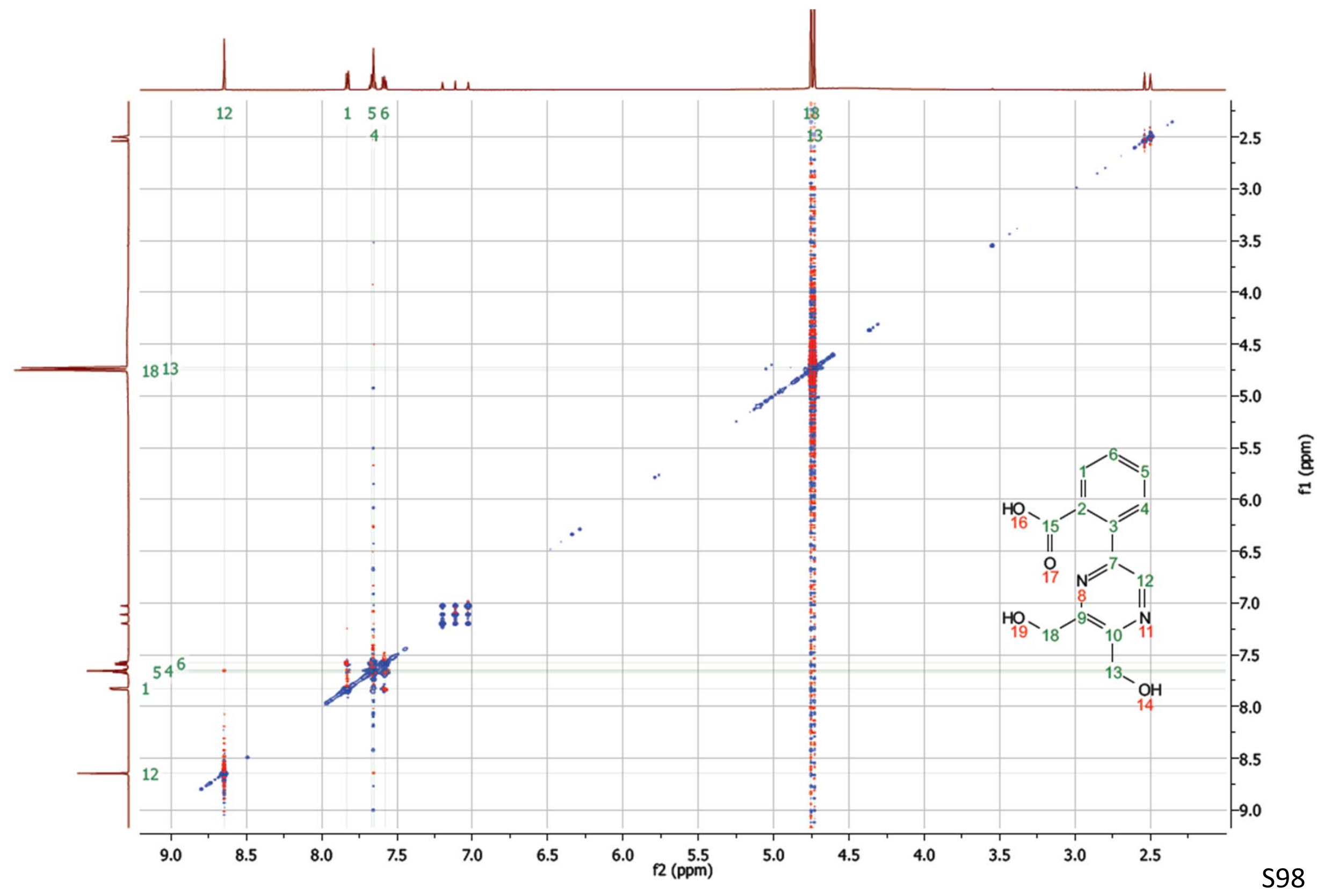




\section{${ }^{1} \mathrm{H}$ NMR spectrum of $12 \mathrm{a} \bullet T F A\left(600 \mathrm{MHz}, \mathrm{CD}_{3} \mathrm{OD}\right)$}

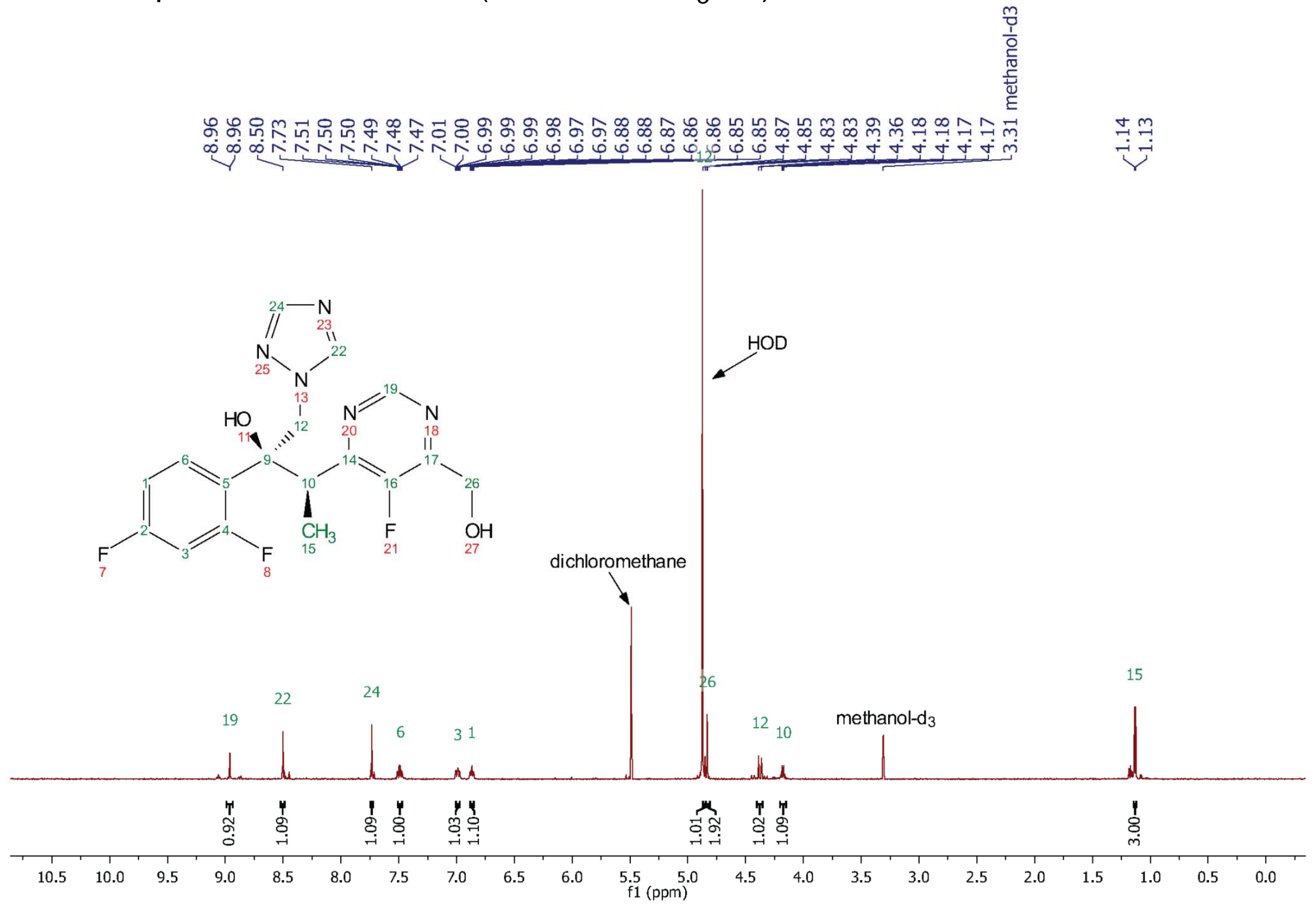


${ }^{13} \mathrm{C}$ NMR spectrum of 12a•TFA (150 MHz, $\left.\mathrm{CD}_{3} \mathrm{OD}\right)$
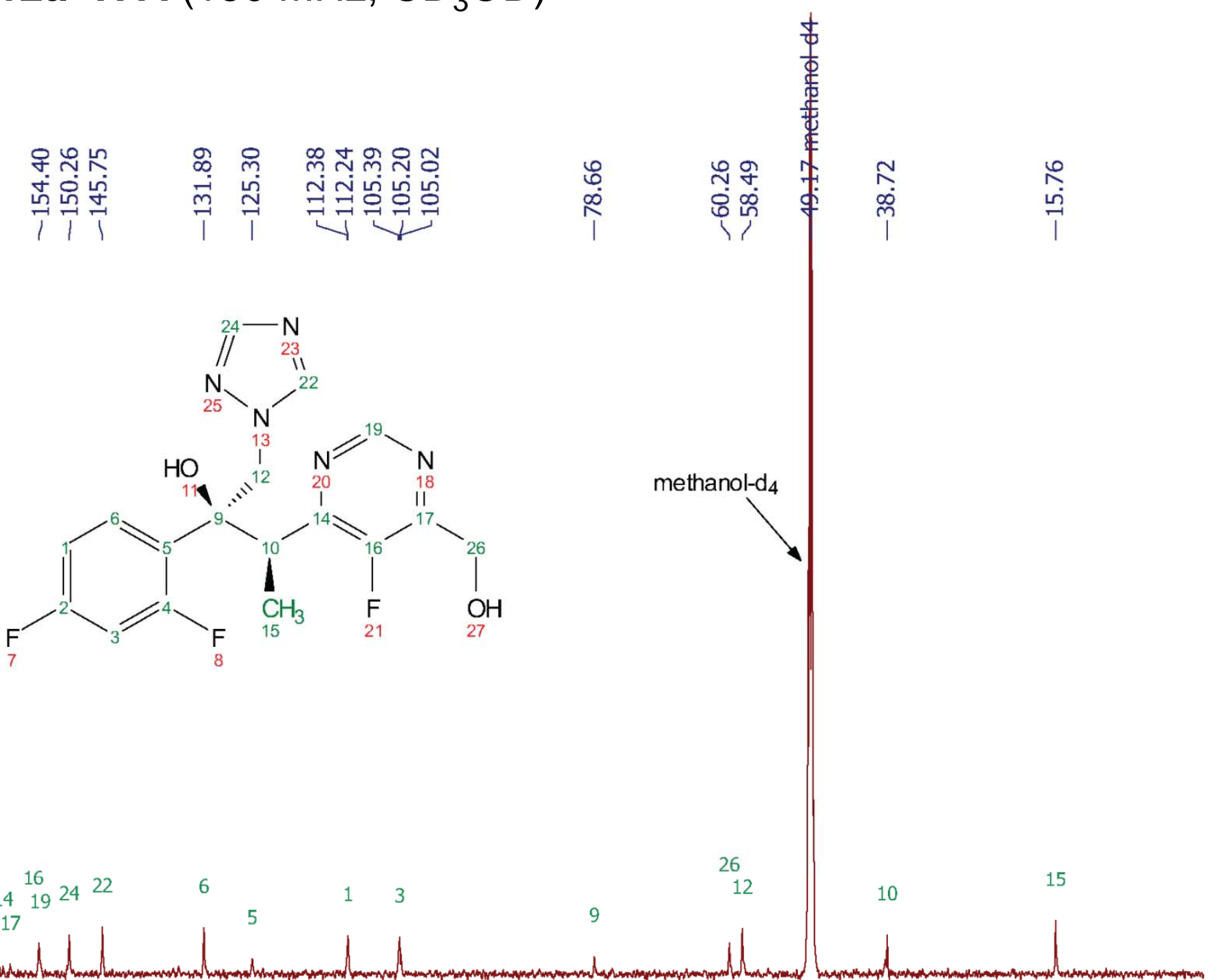

$210 \quad 200 \quad 190 \quad 180$
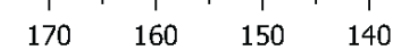

130

120

110100

80

60

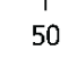

40

30 
${ }^{19} \mathrm{~F}$ NMR spectrum of 12a•TFA (564 MHz, $\mathrm{CD}_{3} \mathrm{OD}$ )

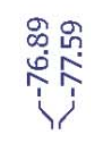

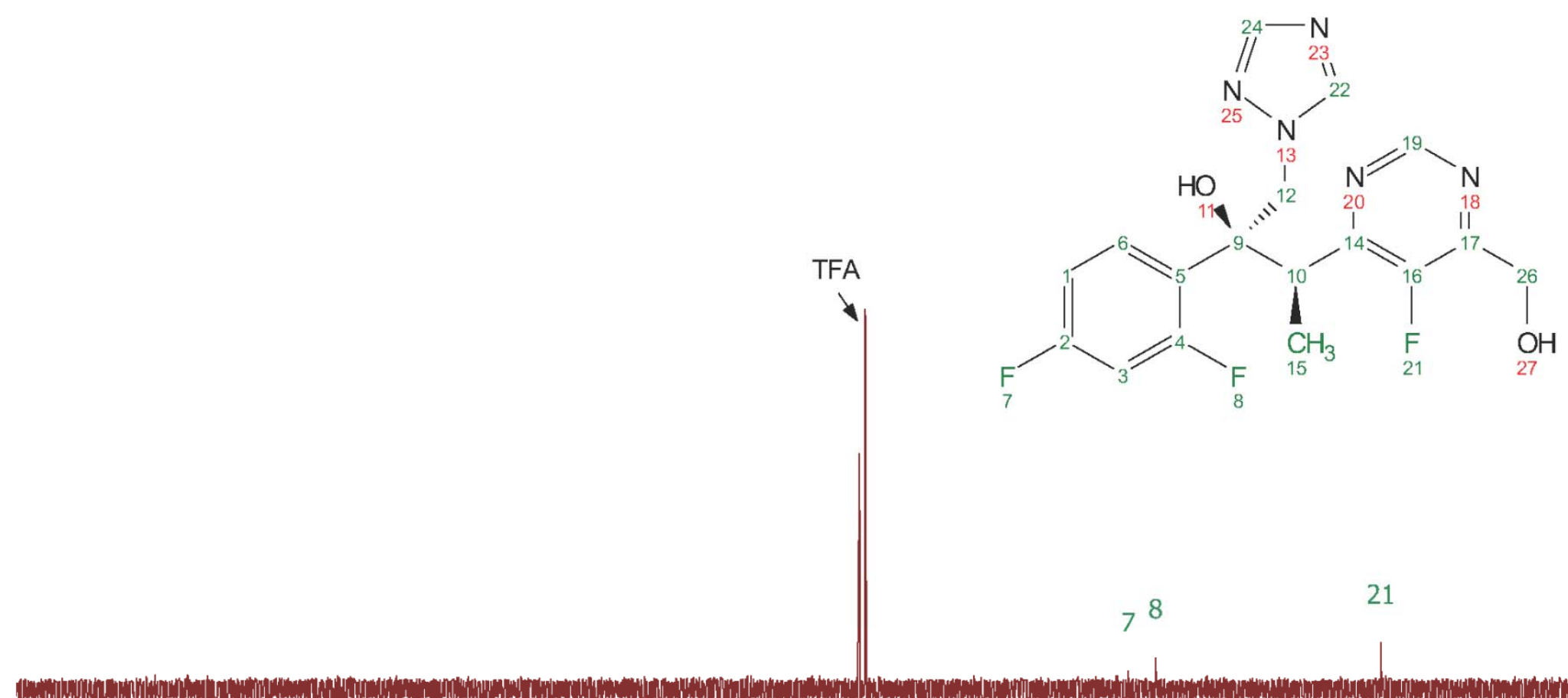

\begin{tabular}{|c|c|c|c|c|c|c|c|c|c|c|c|c|c|c|c|c|c|c|c|c|}
\hline 20 & 10 & 0 & -10 & -20 & $\begin{array}{l}1 \\
-30\end{array}$ & -40 & $\begin{array}{c}1 \\
-50\end{array}$ & -60 & $\begin{array}{l}1 \\
-70\end{array}$ & $\begin{array}{ll}-80 & -90 \\
f 1(p p m)\end{array}$ & -100 & -110 & -120 & -130 & -140 & $\begin{array}{l} \\
-150\end{array}$ & -160 & -170 & -180 & $\begin{array}{l}-190 \\
\text { S101 }\end{array}$ \\
\hline
\end{tabular}


${ }^{1} \mathrm{H} /{ }^{1} \mathrm{H}$ COSY NMR spectrum of 12a・TFA $\left(600 / 600 \mathrm{MHz}, \mathrm{CD}_{3} \mathrm{OD}\right)$

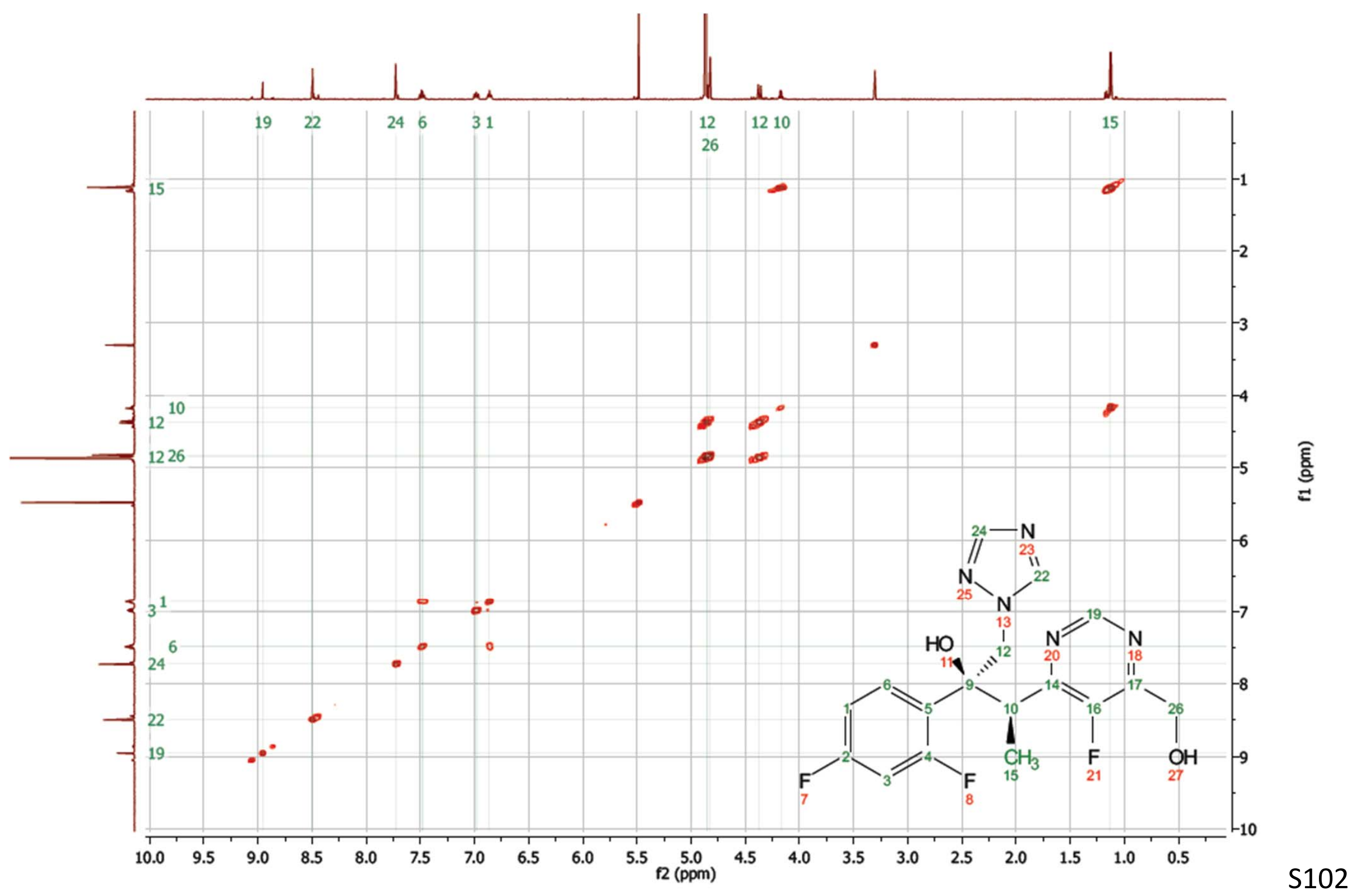


${ }^{1} \mathrm{H} /{ }^{13} \mathrm{C}$ HSQC NMR spectrum of 12a•TFA (600/150 MHz, $\mathrm{CD}_{3} \mathrm{OD}$ )

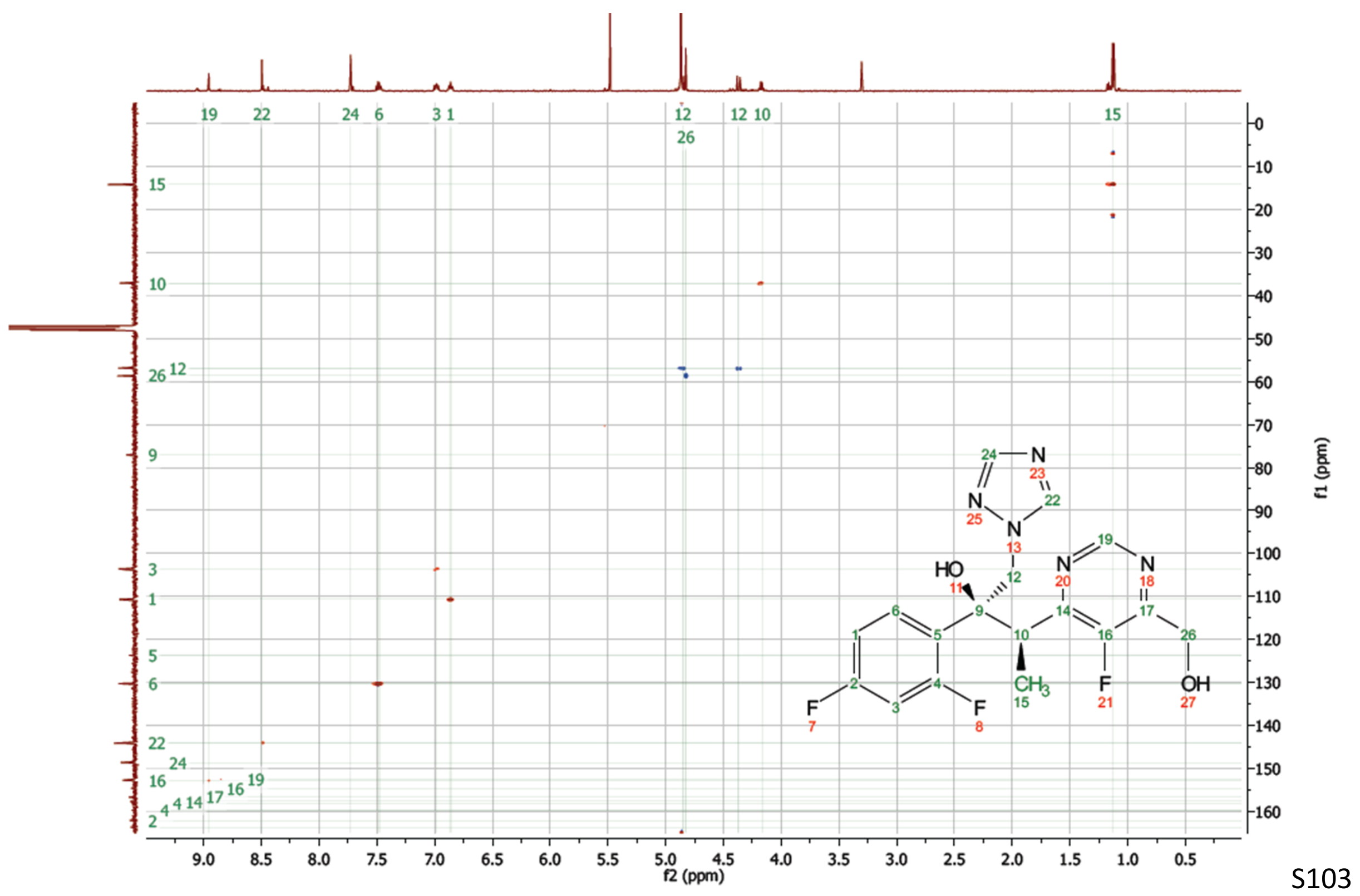


${ }^{1} \mathrm{H} /{ }^{13} \mathrm{C}$ HMBC NMR spectrum of 12a•TFA (600 / $150 \mathrm{MHz}, \mathrm{CD}_{3} \mathrm{OD}$ )

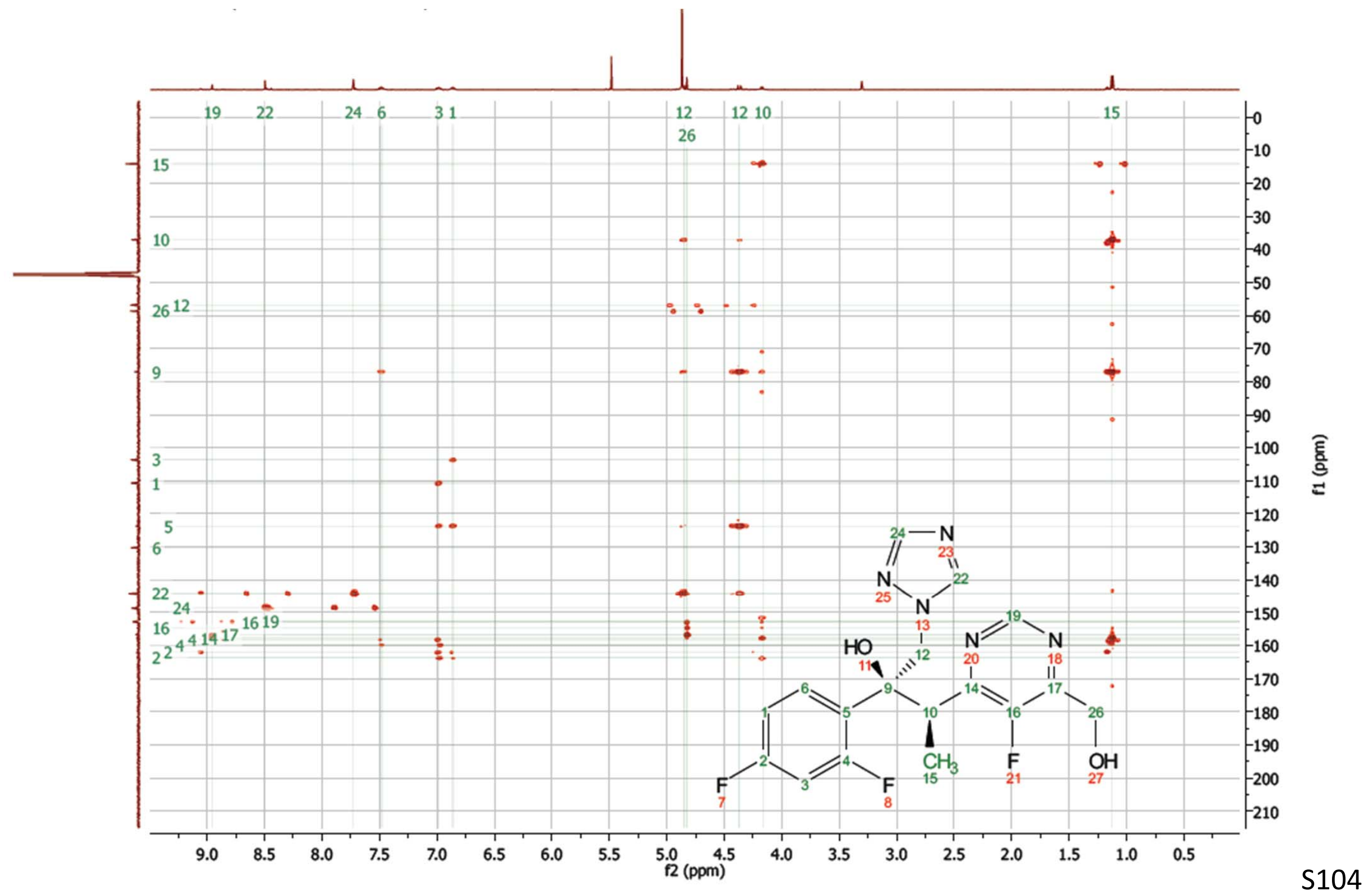


${ }^{1} \mathrm{H} /{ }^{1} \mathrm{H}$ ROESY of $12 \mathrm{a} \cdot T F A\left(600 / 600 \mathrm{MHz}, \mathrm{CD}_{3} \mathrm{OD}\right)$

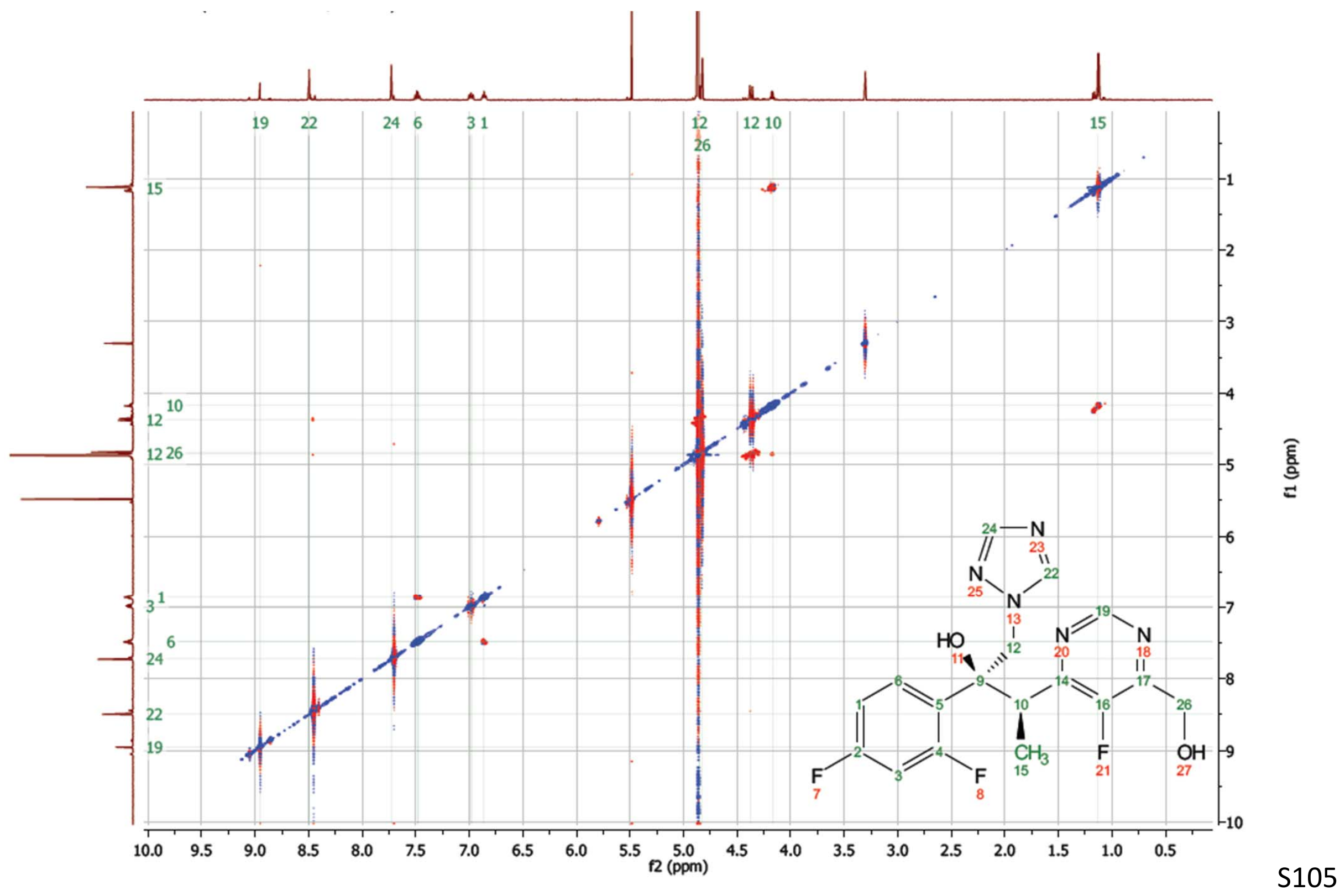




\section{${ }^{1} \mathrm{H}$ NMR spectrum of $12 \mathrm{~b} \bullet T F A\left(600 \mathrm{MHz}, \mathrm{CD}_{3} \mathrm{OD}\right)$}

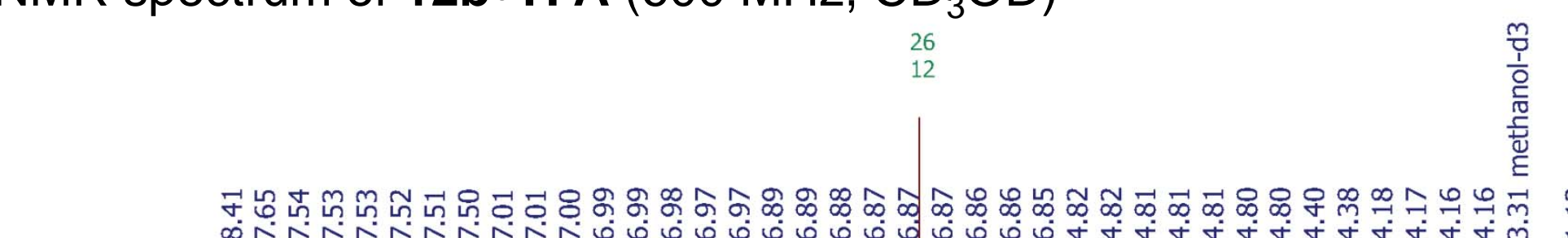

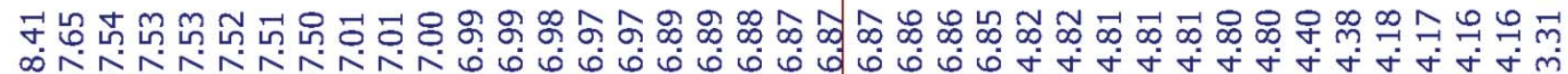

艺

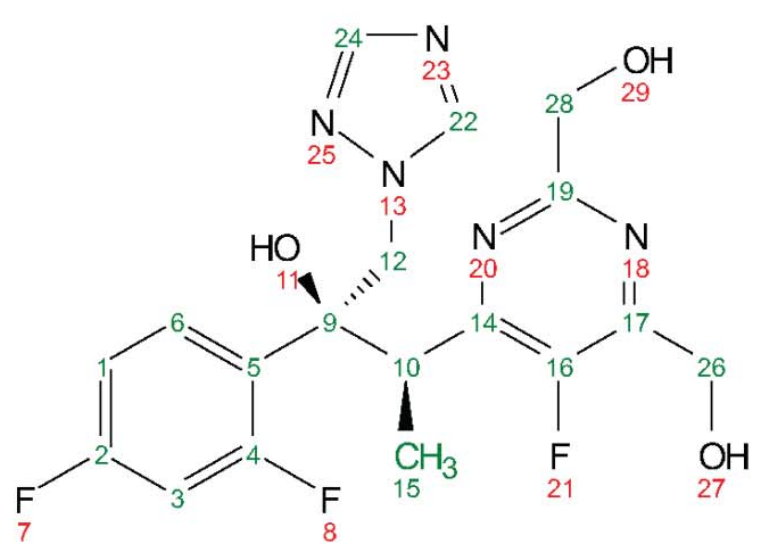

\section{dichloromethane}

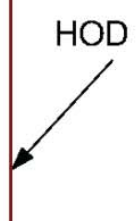

15
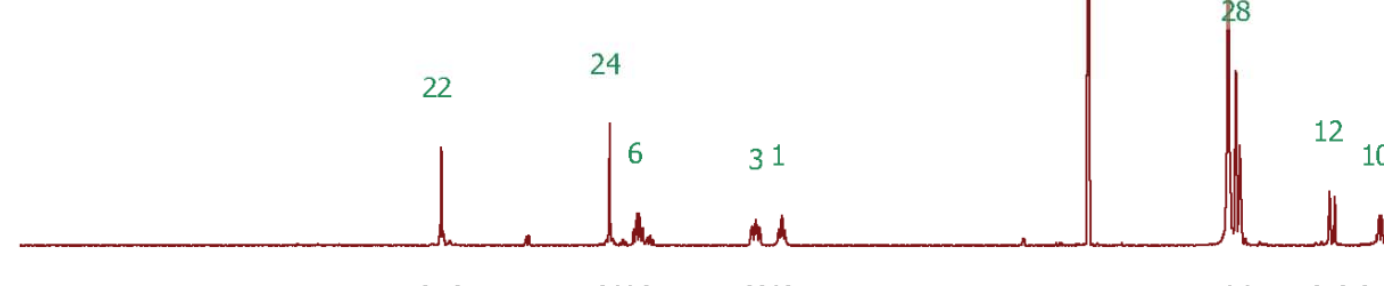

\section{methanol- $\mathrm{d}_{3}$}

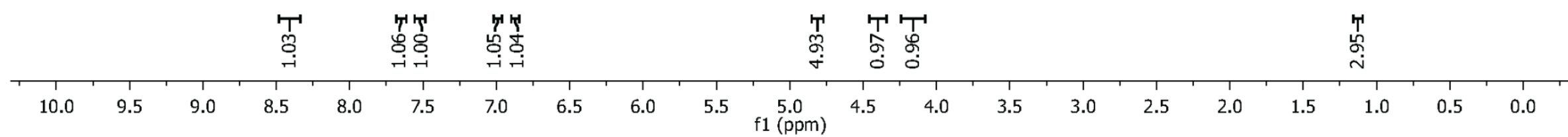


${ }^{13} \mathrm{C}$ NMR spectrum of 12b•TFA (150 MHz, CD 3 OD)

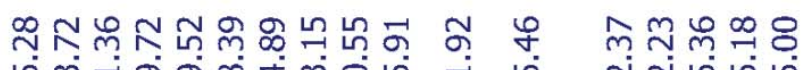

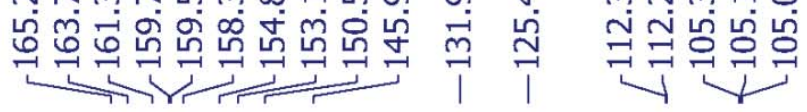

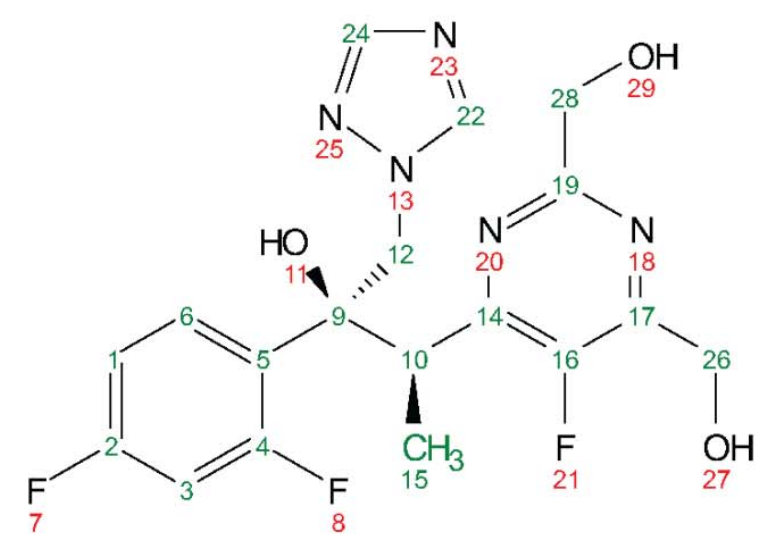

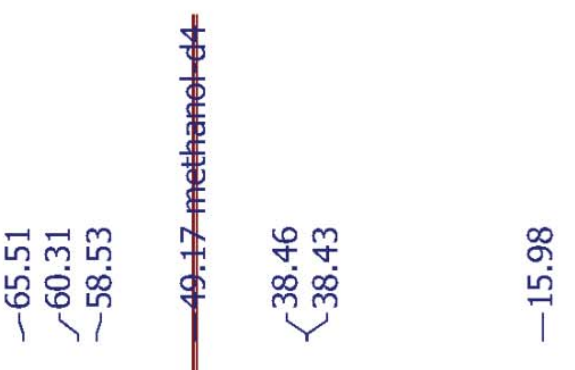

15

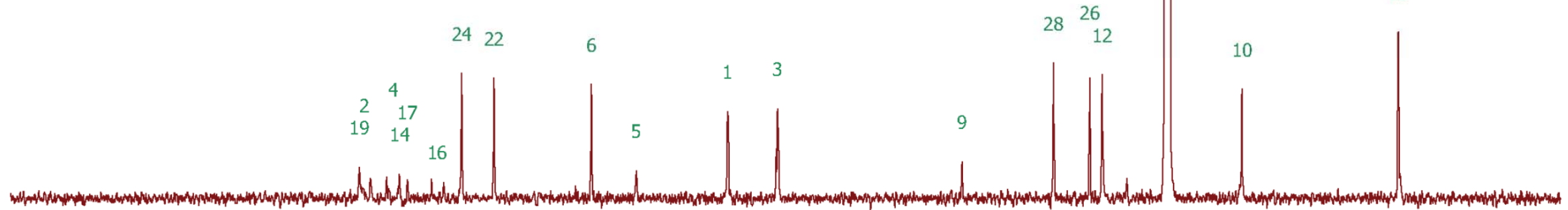

$210 \quad 200$

190

170

160

$150 \quad 140$

120

110100

90

80 
${ }^{19} \mathrm{~F}$ NMR spectrum of $12 \mathrm{~b} \bullet$ TFA $\left(470 \mathrm{MHz}, \mathrm{CD}_{3} \mathrm{OD}\right)$

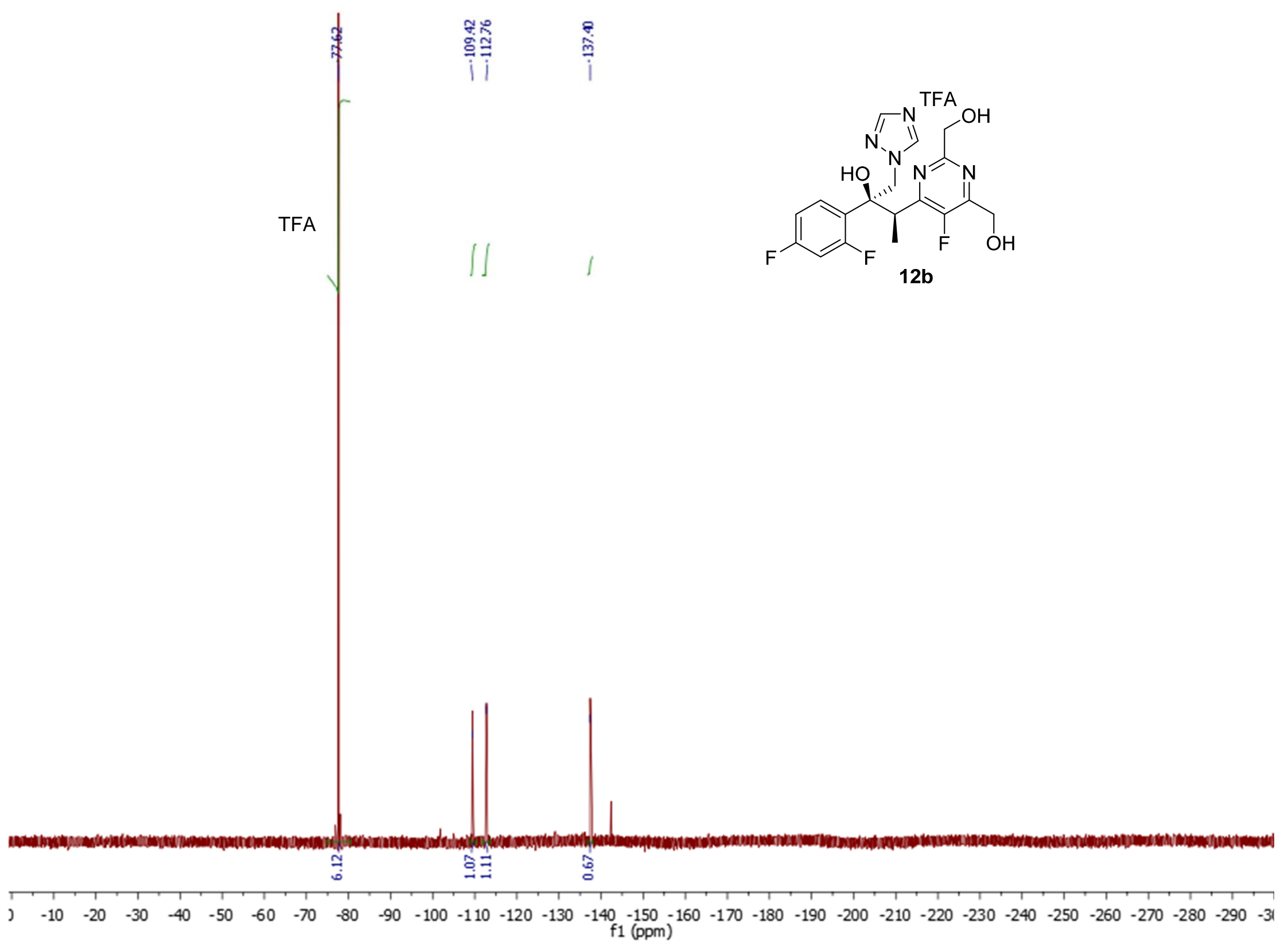


${ }^{1} \mathrm{H} /{ }^{1} \mathrm{H}$ COSY NMR spectrum of 12b•TFA (600/600 MHz, CD $\left.{ }_{3} \mathrm{OD}\right)$

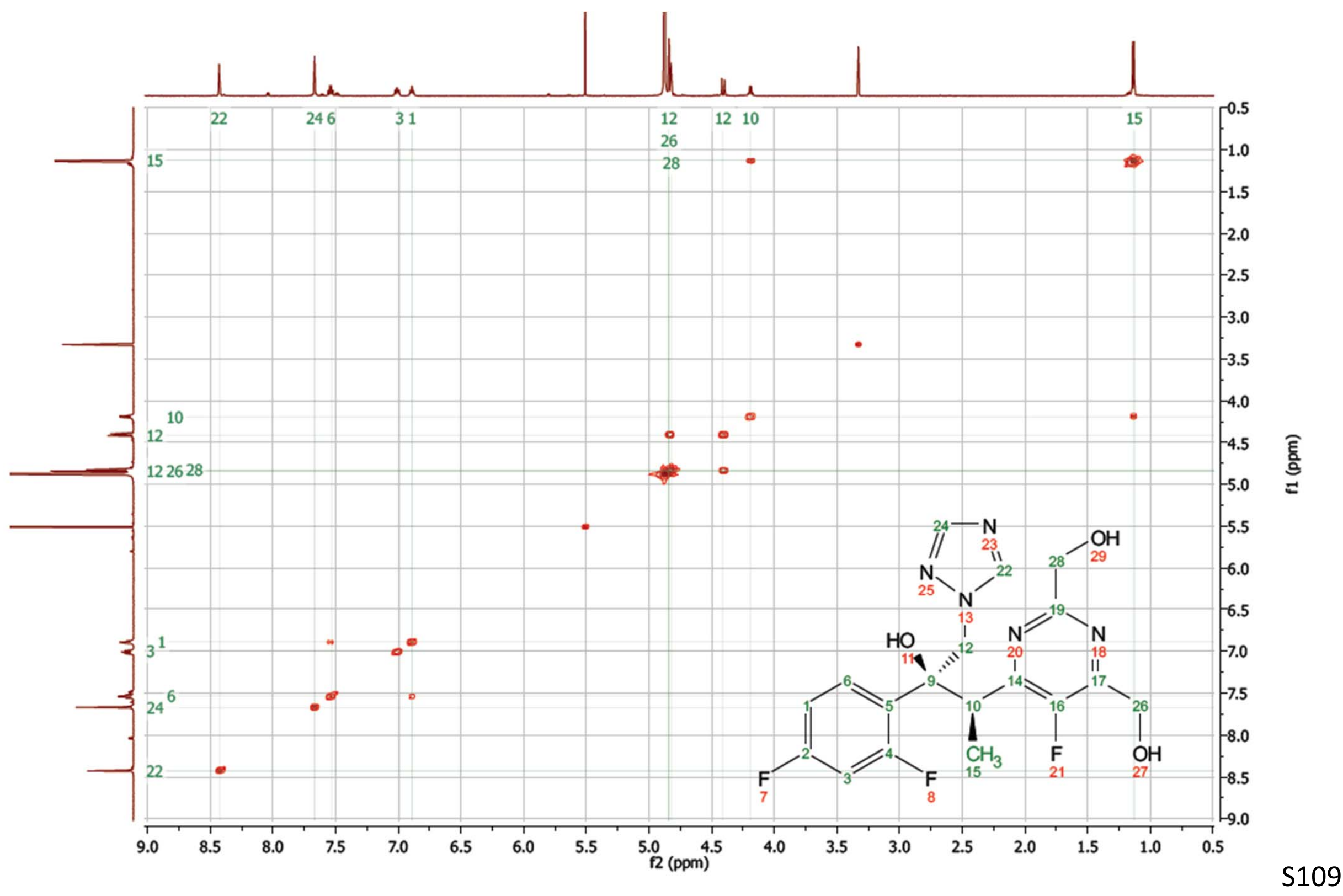


${ }^{1} \mathrm{H} /{ }^{13} \mathrm{C}$ HSQC of 12b•TFA (600 / $\left.150 \mathrm{MHz}, \mathrm{CD}_{3} \mathrm{OD}\right)$

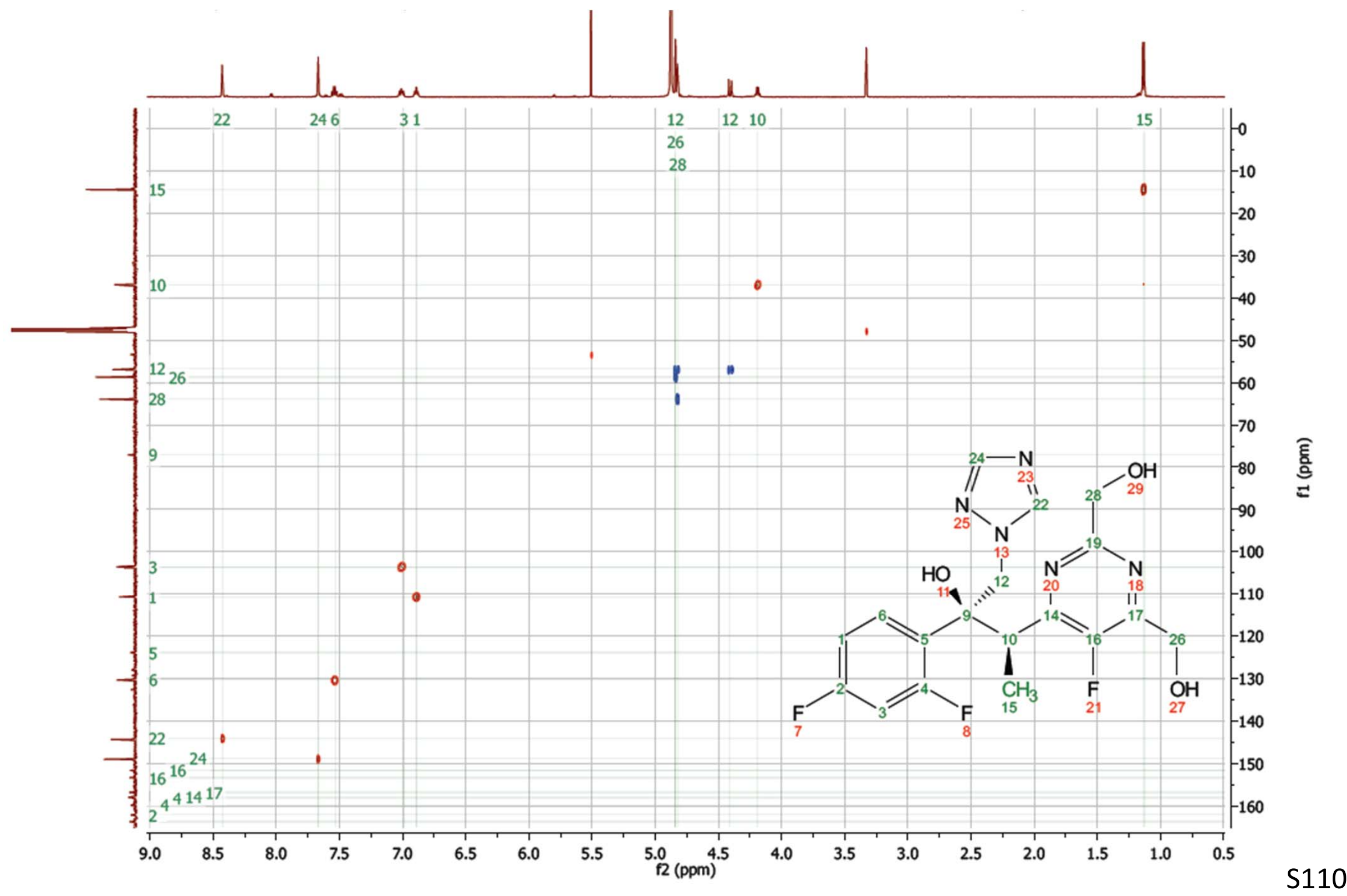


${ }^{1} \mathrm{H} /{ }^{13} \mathrm{C}$ HMBC NMR spectrum of 12b•TFA (600 / $150 \mathrm{MHz}, \mathrm{CD}_{3} \mathrm{OD}$ )

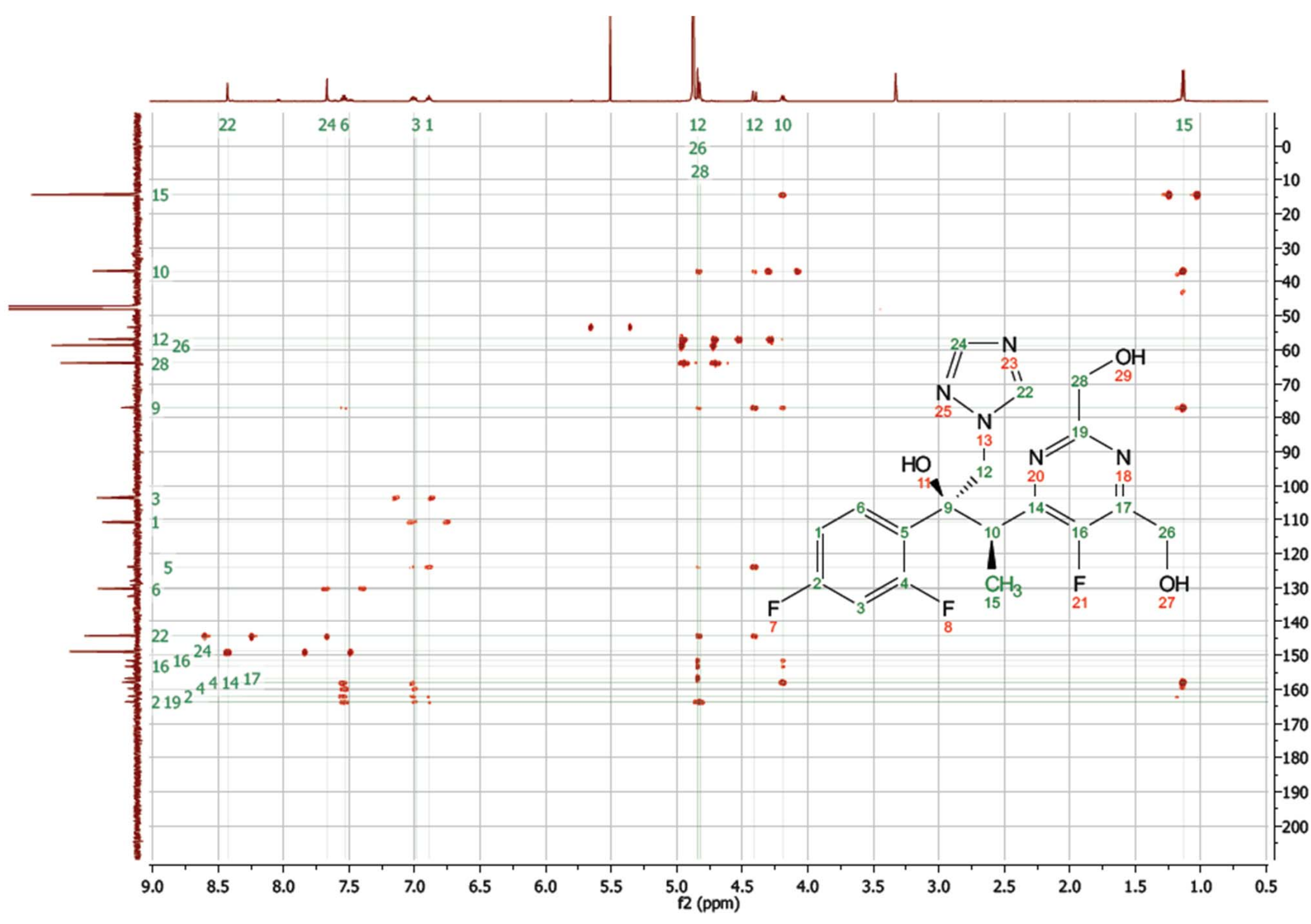


${ }^{1} \mathrm{H} /{ }^{1} \mathrm{H}$ ROESY NMR spectrum of 12b•TFA (600 / $600 \mathrm{MHz}, \mathrm{CD}_{3} \mathrm{OD}$ )

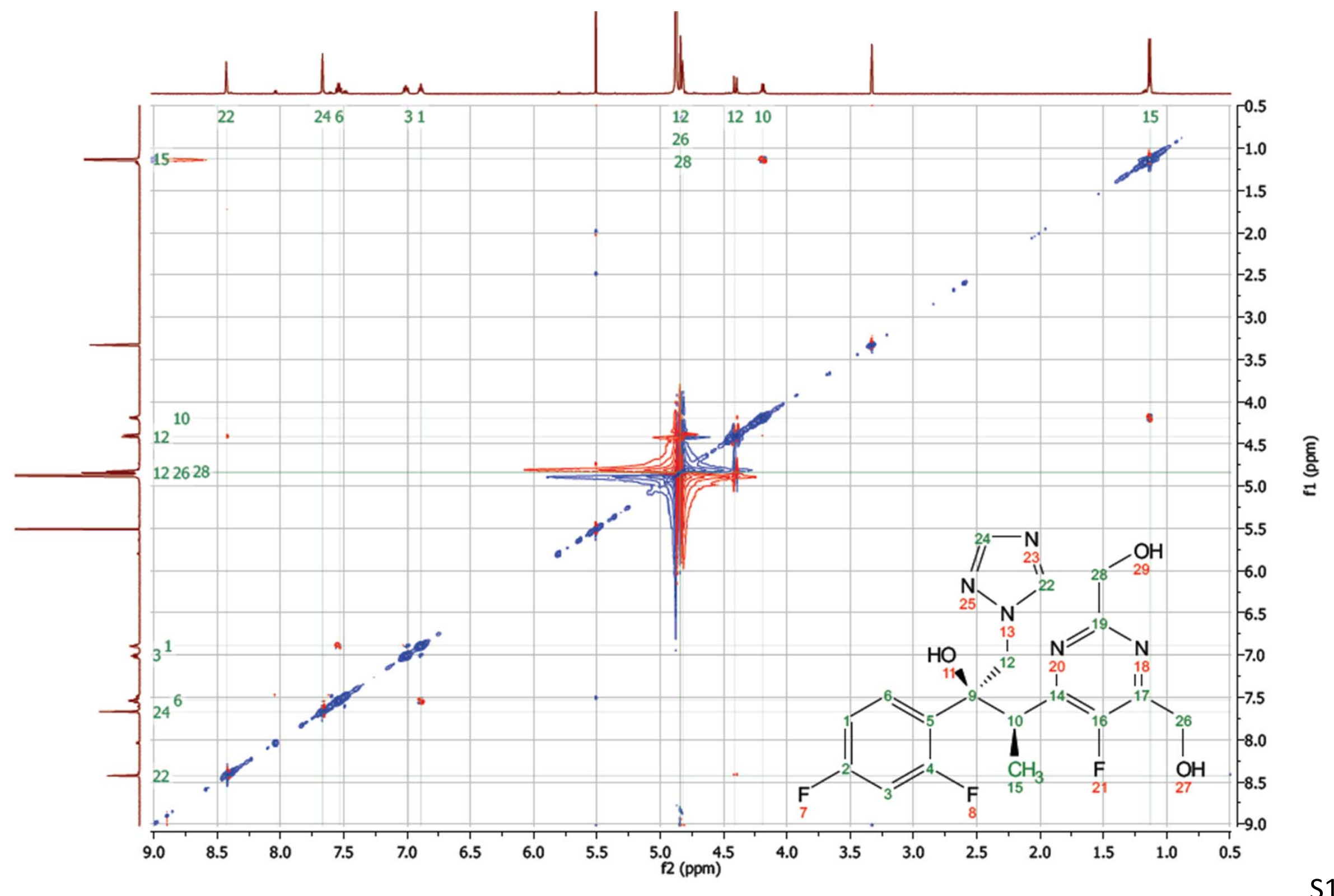


${ }^{1} \mathrm{H} /{ }^{15} \mathrm{~N} \mathrm{HMBC}$ NMR spectrum of 12b•TFA $\left(600 / 61 \mathrm{MHz}, \mathrm{CD}_{3} \mathrm{OD}\right)$

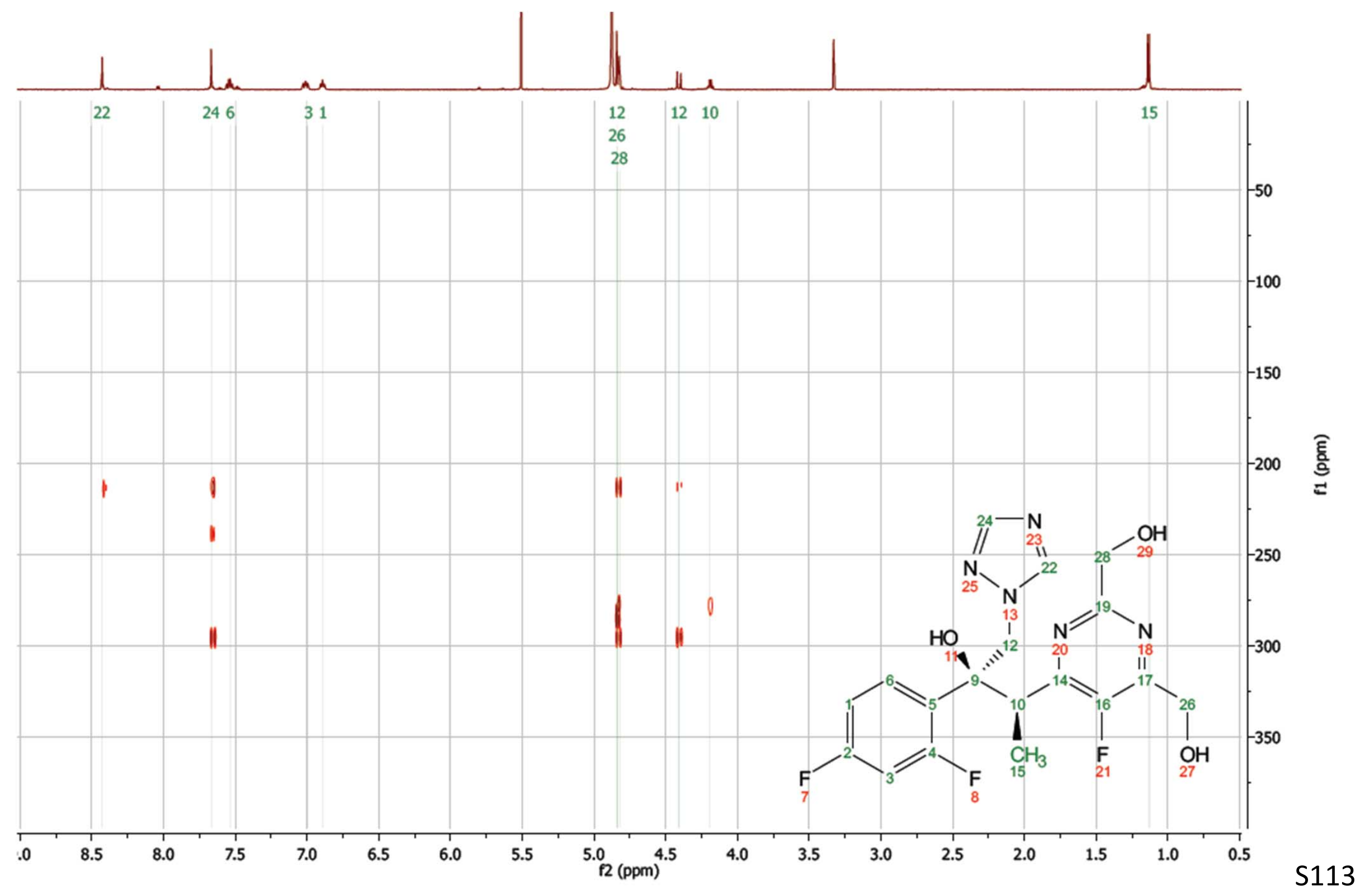


${ }^{1} \mathrm{H}$ NMR spectrum of $13 \mathrm{a} \cdot \mathrm{TFA}\left(500 \mathrm{MHz}, \mathrm{CD}_{3} \mathrm{OD}\right.$ )
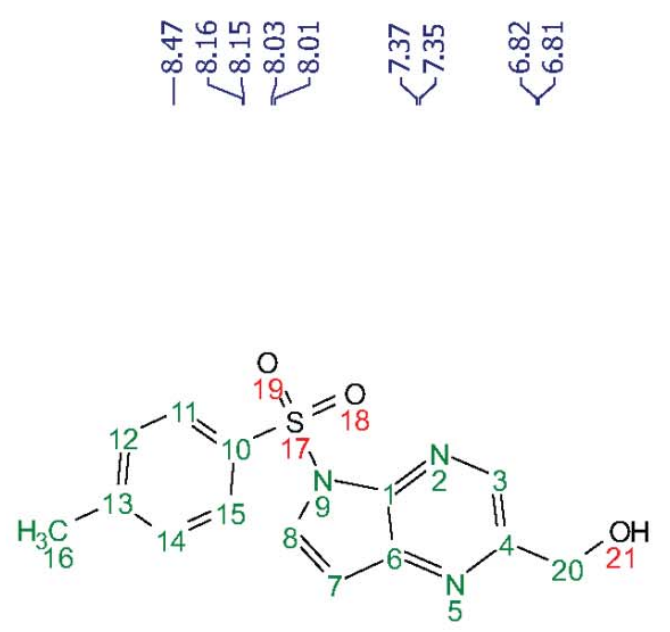

3
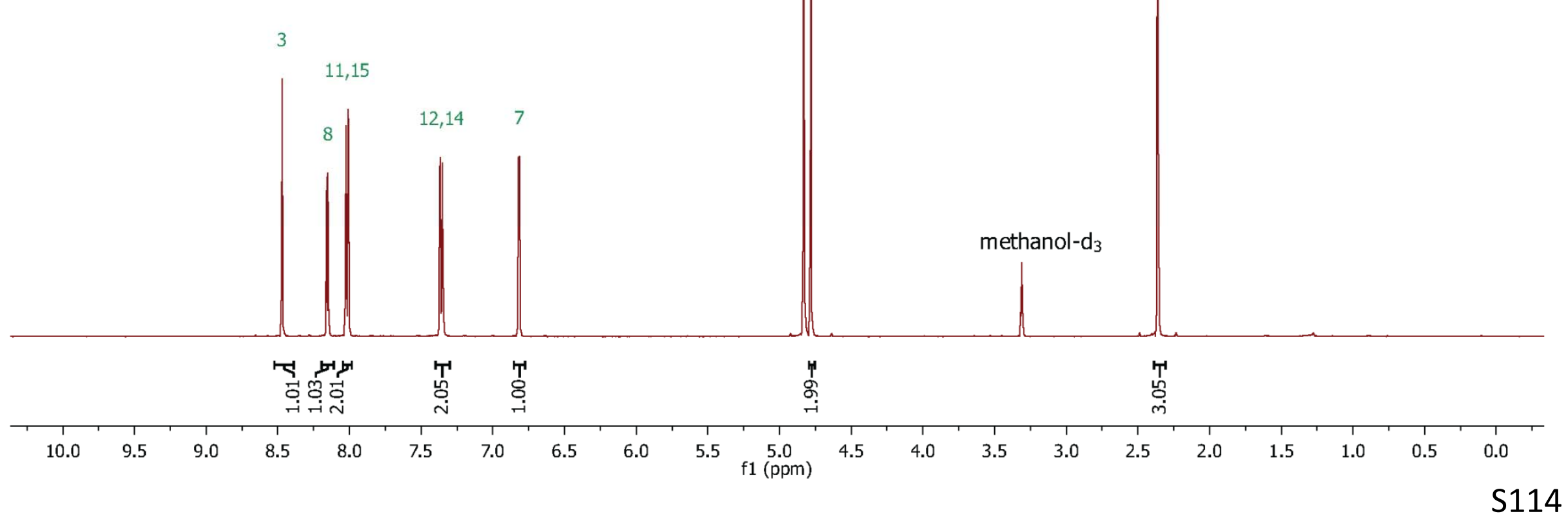
${ }^{13} \mathrm{C}$ NMR spectrum of 13a•TFA (125 MHz, $\left.\mathrm{CD}_{3} \mathrm{OD}\right)$
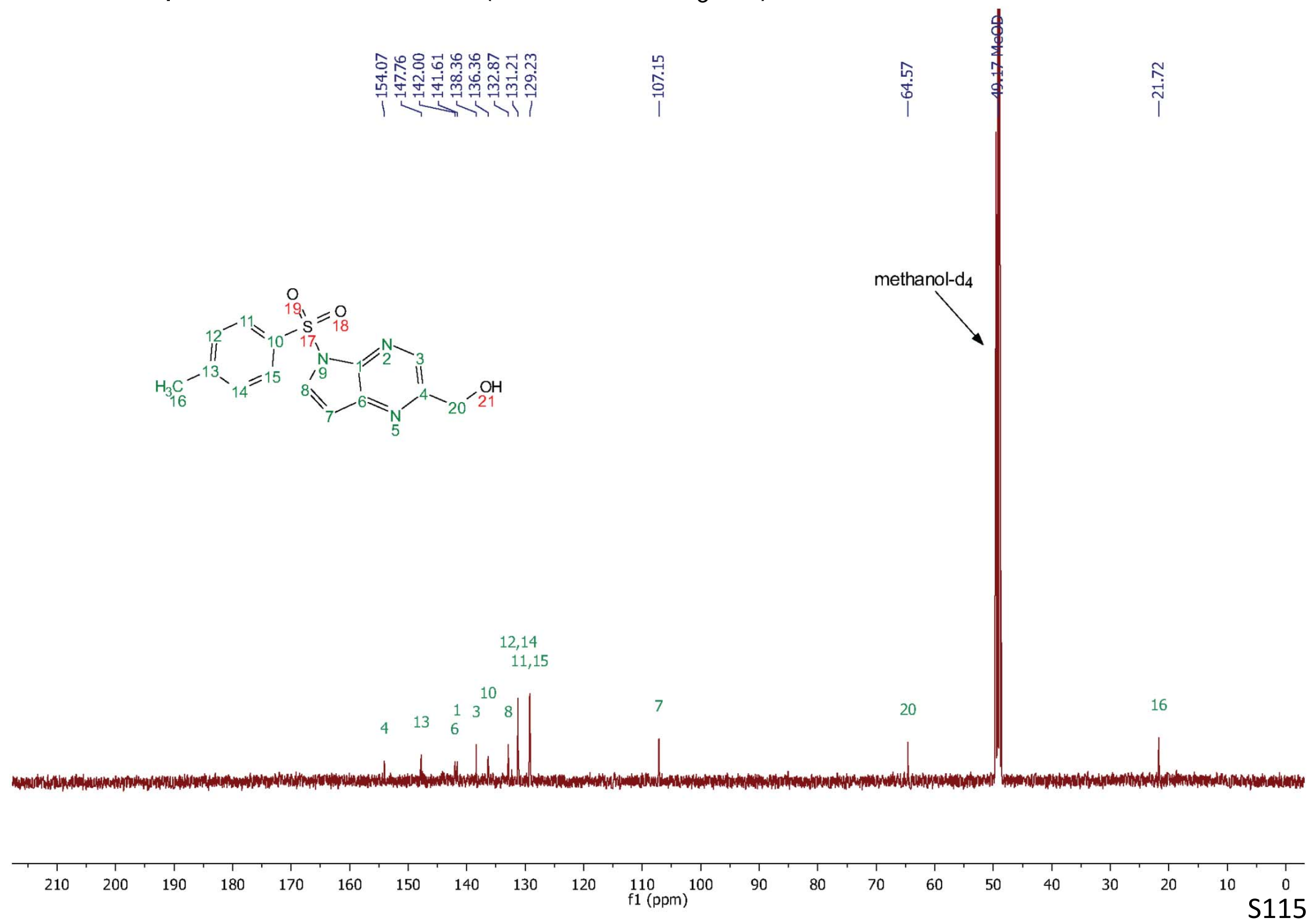
${ }^{1} \mathrm{H} /{ }^{1} \mathrm{H}$ COSY NMR spectrum of 13a・TFA $\left(500 / 500 \mathrm{MHz}, \mathrm{CD}_{3} \mathrm{OD}\right)$

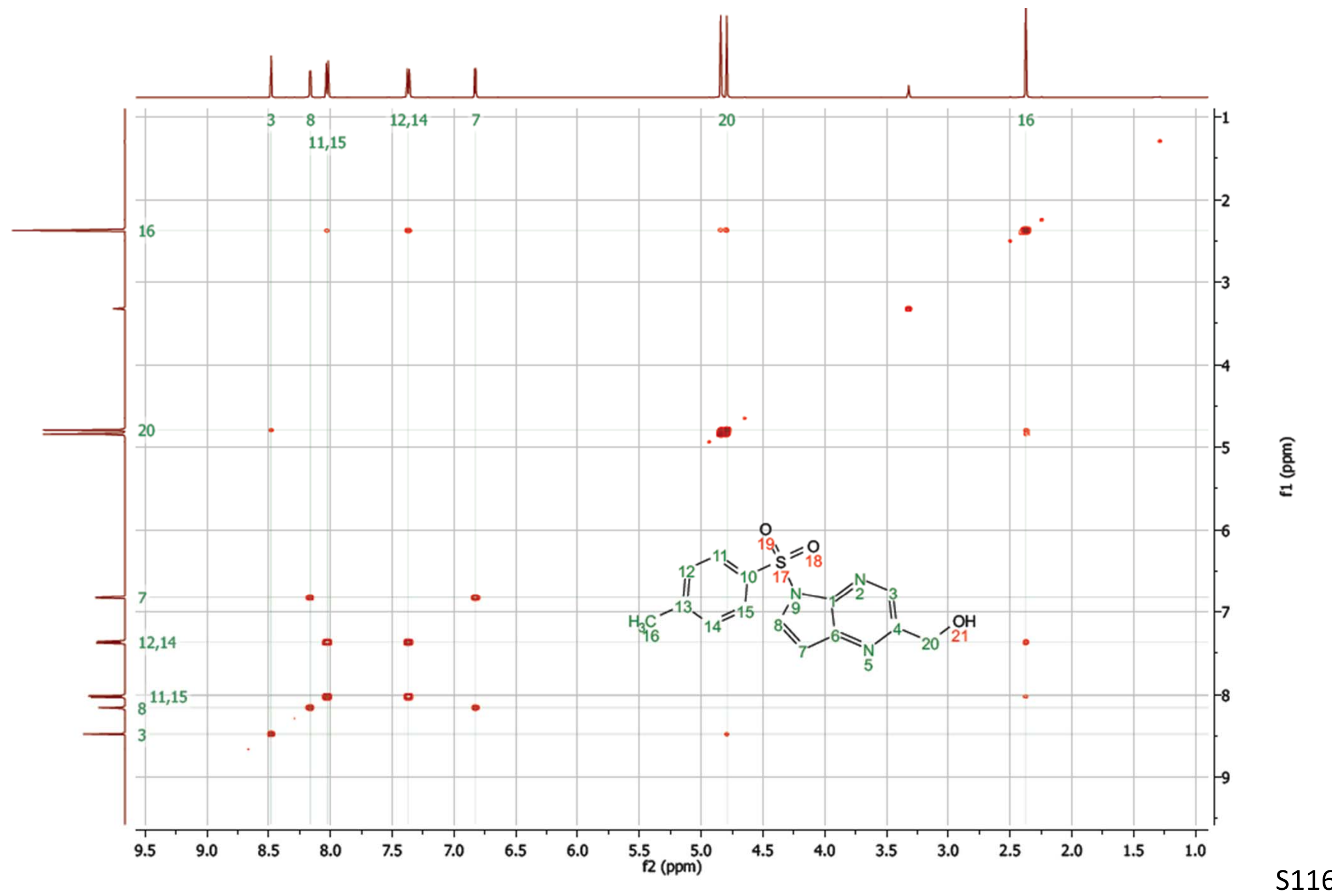


${ }^{1} \mathrm{H} /{ }^{13} \mathrm{C}$ HSQC NMR spectrum of 13a•TFA (500/125 MHz, $\mathrm{CD}_{3} \mathrm{OD}$ )

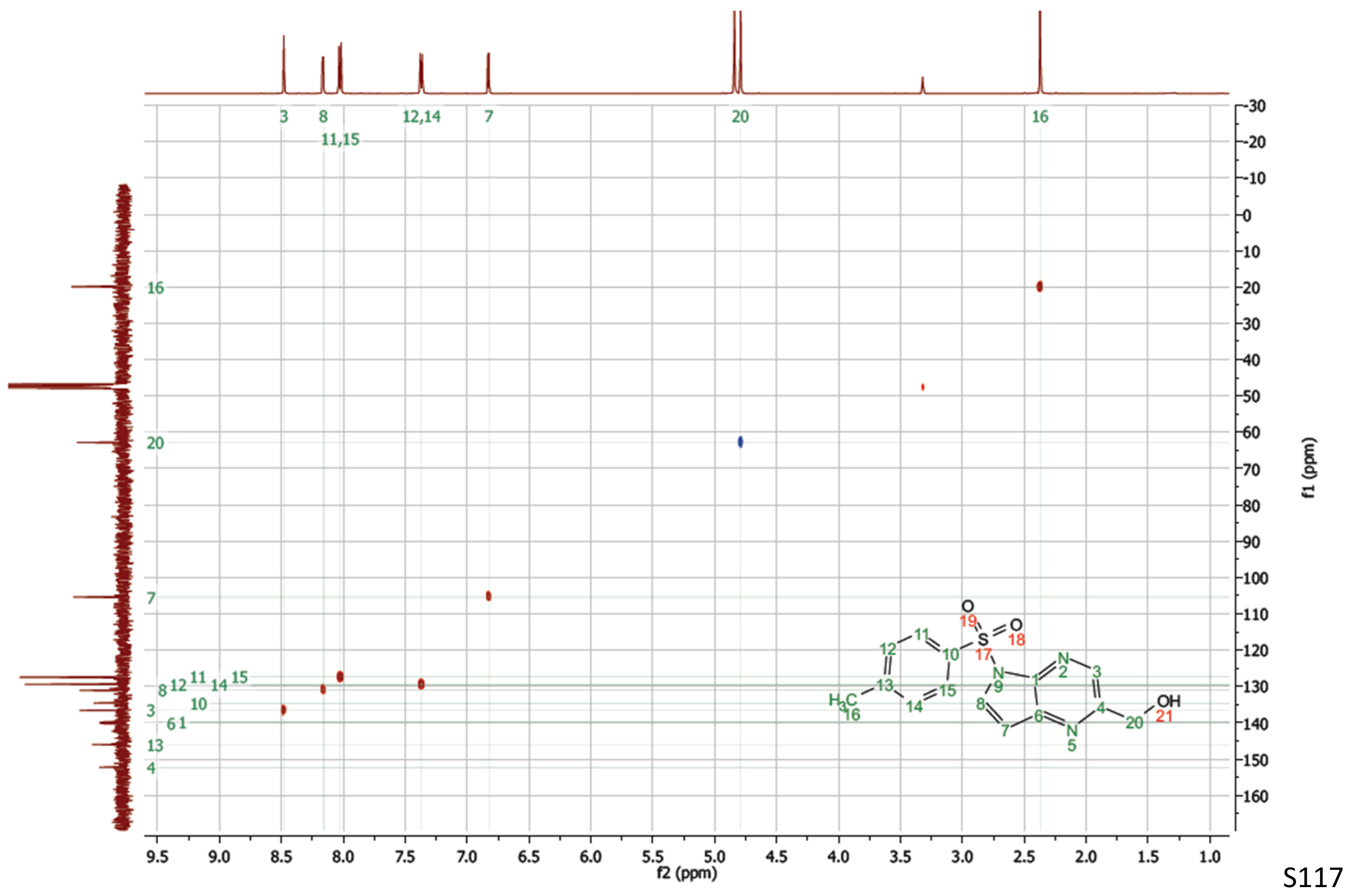


${ }^{1} \mathrm{H} /{ }^{13} \mathrm{C}$ HMBC NMR spectrum of 13a・TFA (500 / $125 \mathrm{MHz}, \mathrm{CD}_{3} \mathrm{OD}$ )

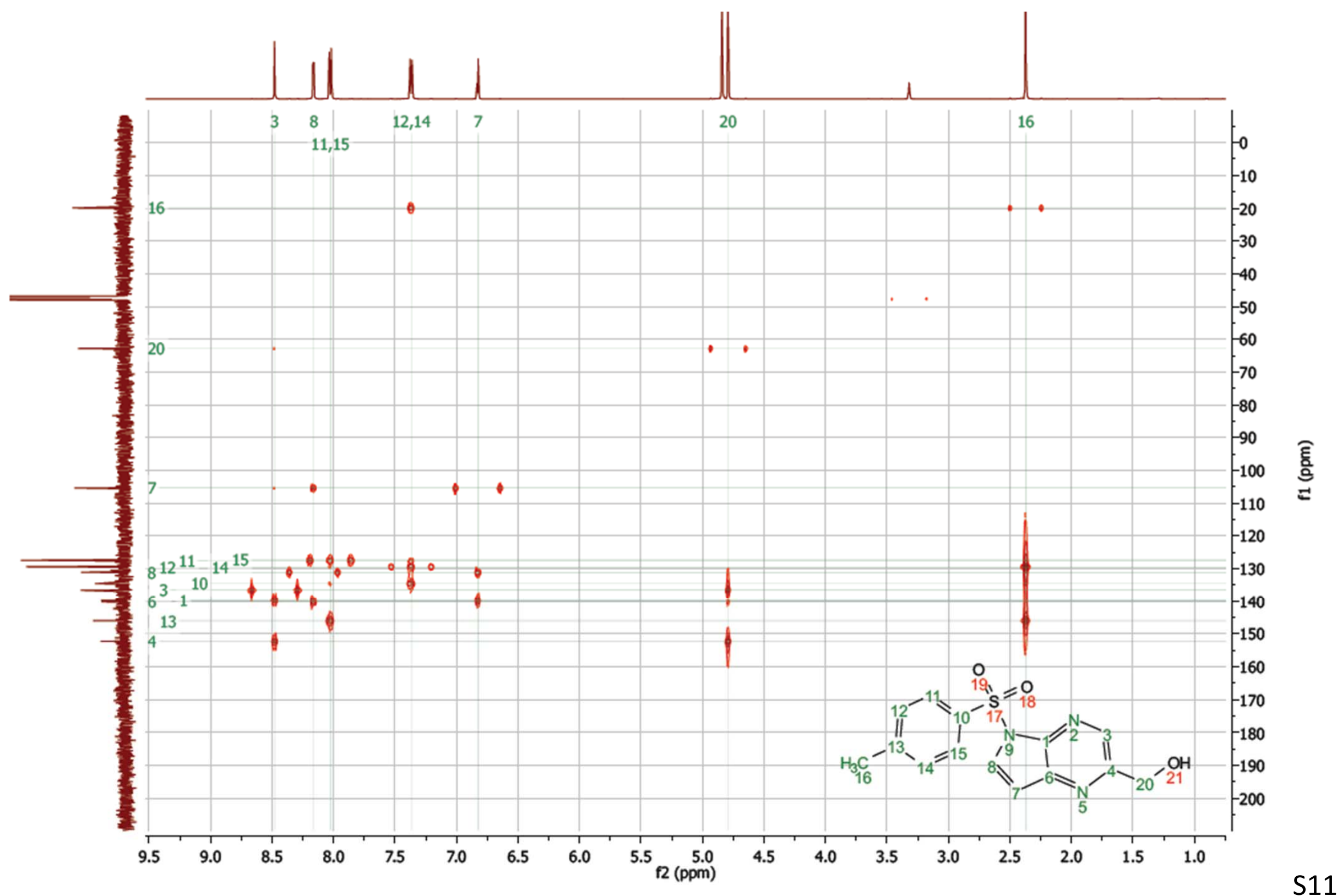


${ }^{1} \mathrm{H} /{ }^{15} \mathrm{~N}$ HMBC NMR spectrum of 13a•TFA (500/51 MHz, $\mathrm{CD}_{3} \mathrm{OD}$ )

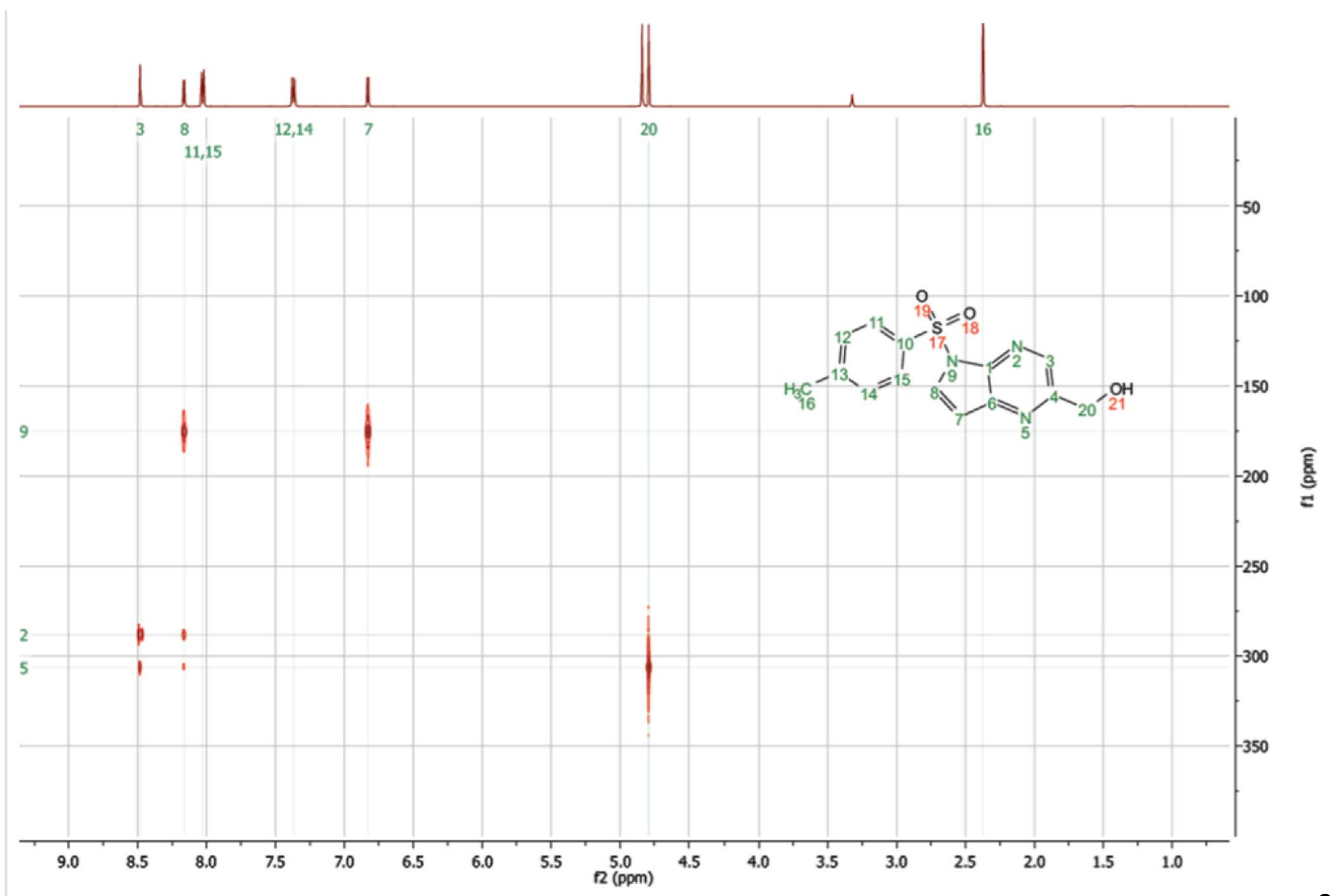


${ }^{1} \mathrm{H}$ NMR spectrum of $14 a\left(500 \mathrm{MHz}, \mathrm{DMSO}-\mathrm{d}_{6}\right)$

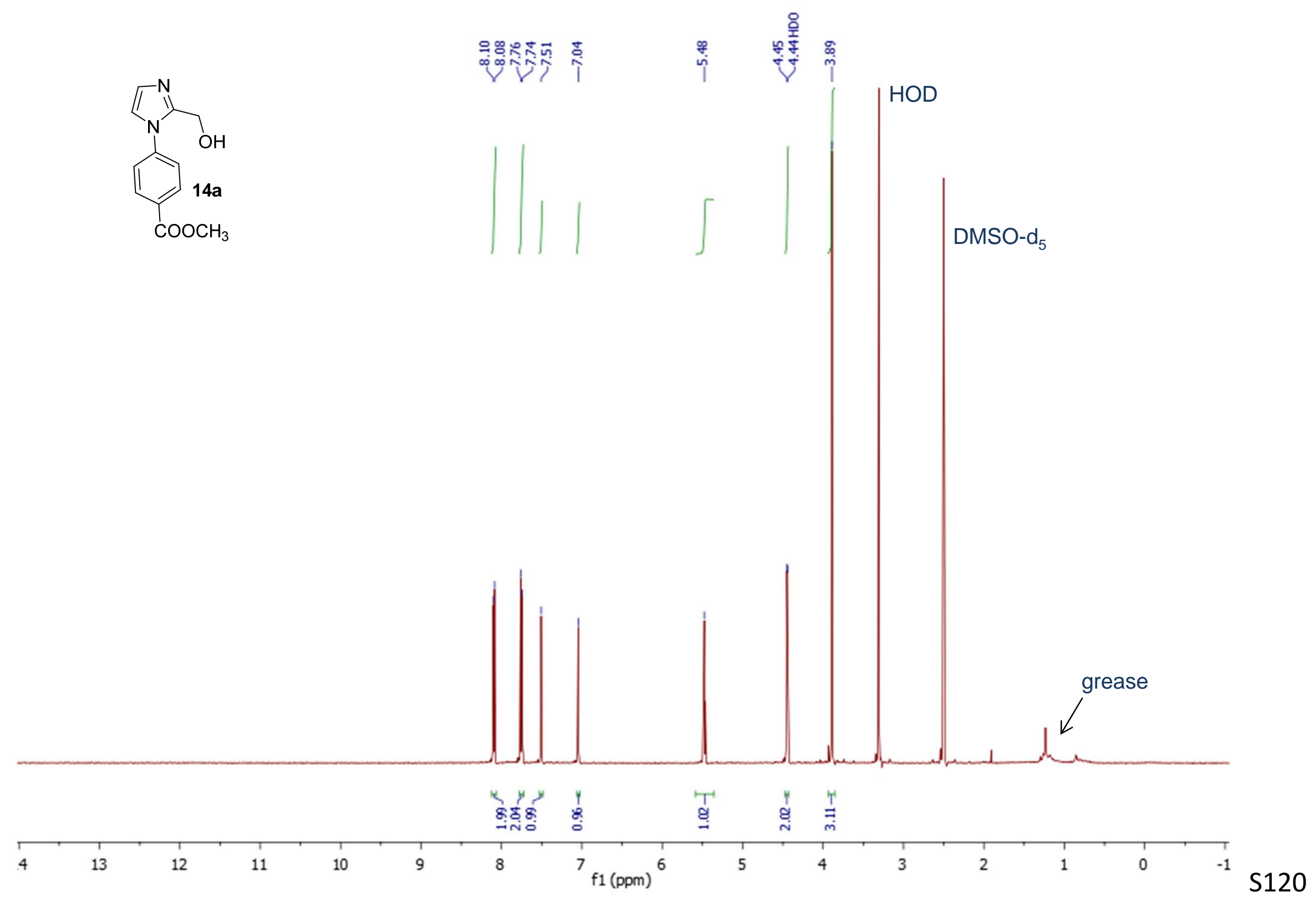


${ }^{13} \mathrm{C}$ NMR spectrum of $14 a\left(125 \mathrm{MHz}\right.$, DMSO-d $\left.\mathrm{d}_{6}\right)$

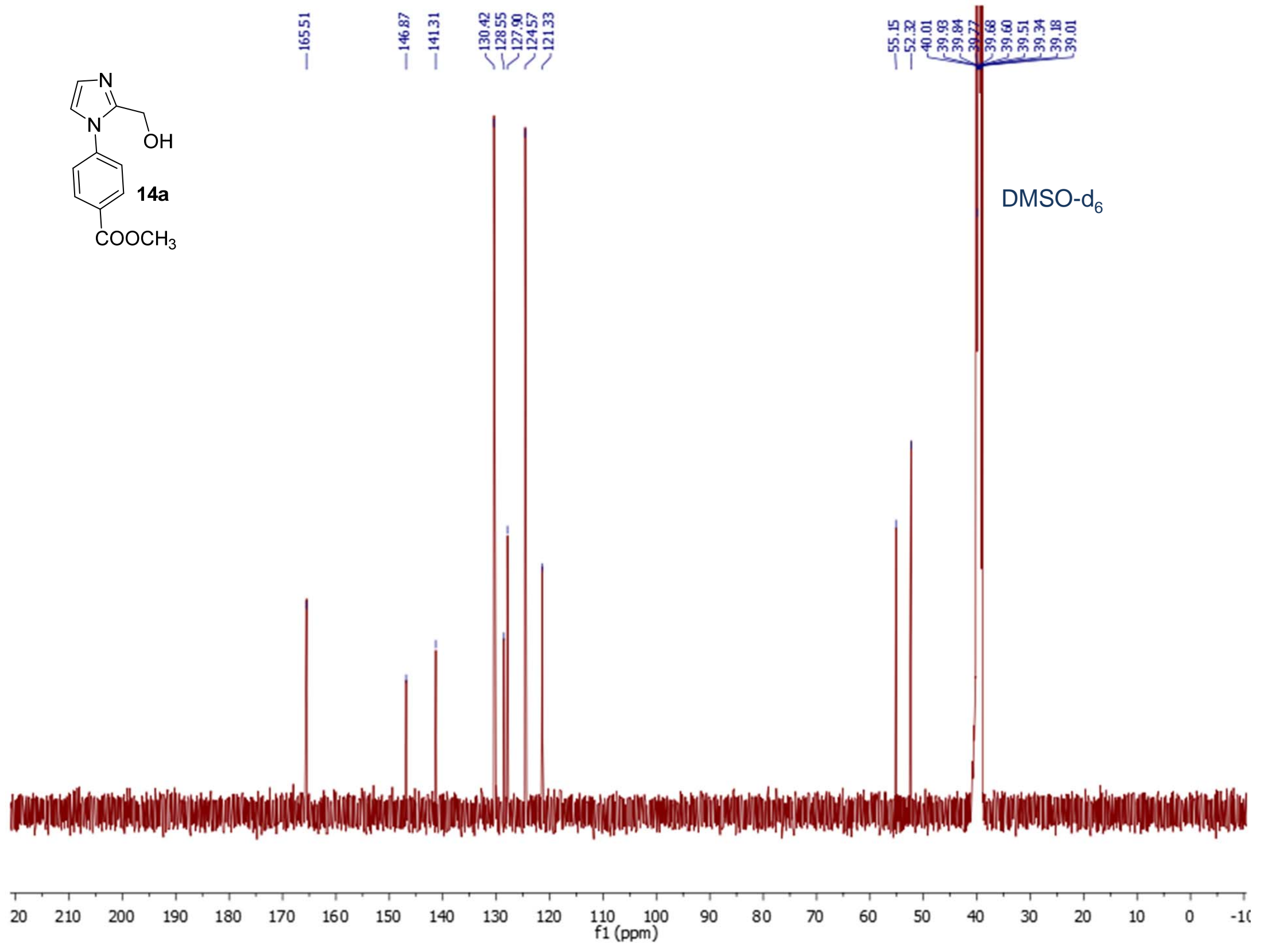


${ }^{1} \mathrm{H}$ NMR spectrum of $\mathbf{1 5 a}\left(600 \mathrm{MHz}\right.$, DMSO-d $\left.\mathrm{d}_{6}\right)$

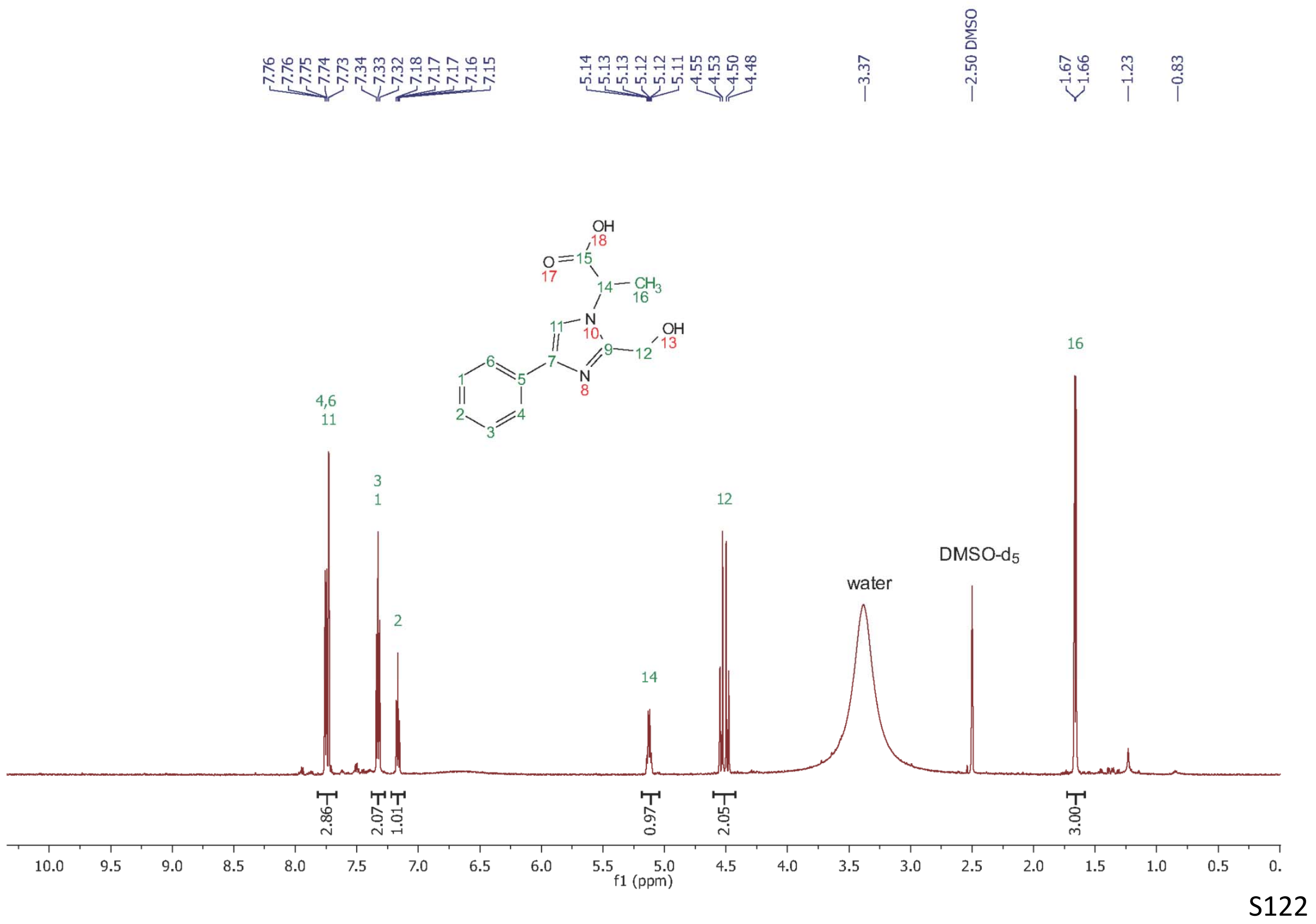


${ }^{13} \mathrm{C}$ NMR spectrum of $15 a\left(150 \mathrm{MHz}, \mathrm{DMSO}-\mathrm{d}_{6}\right)$

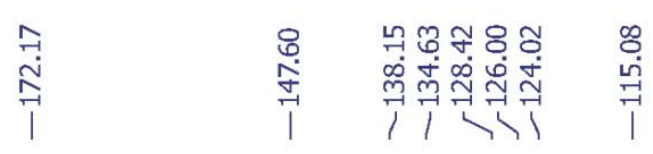
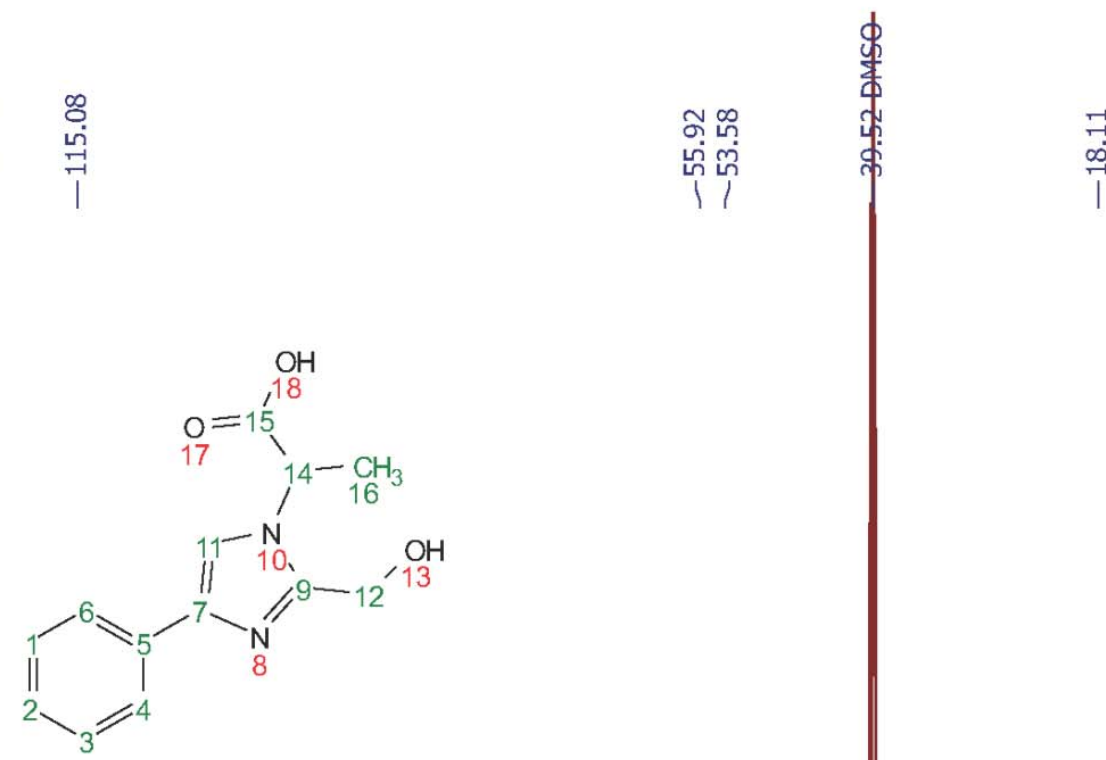

$\underset{\substack{-1 \\-1}}{-1}$

16

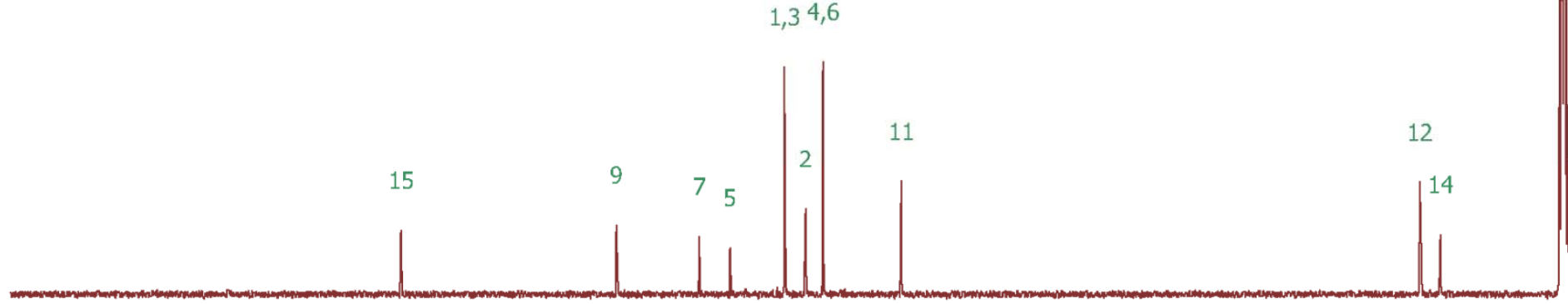


${ }^{1} \mathrm{H} /{ }^{1} \mathrm{H}$ COSY NMR spectrum of $15 \mathrm{a}\left(600 / 600 \mathrm{MHz}\right.$, DMSO-d $\left.\mathrm{d}_{6}\right)$

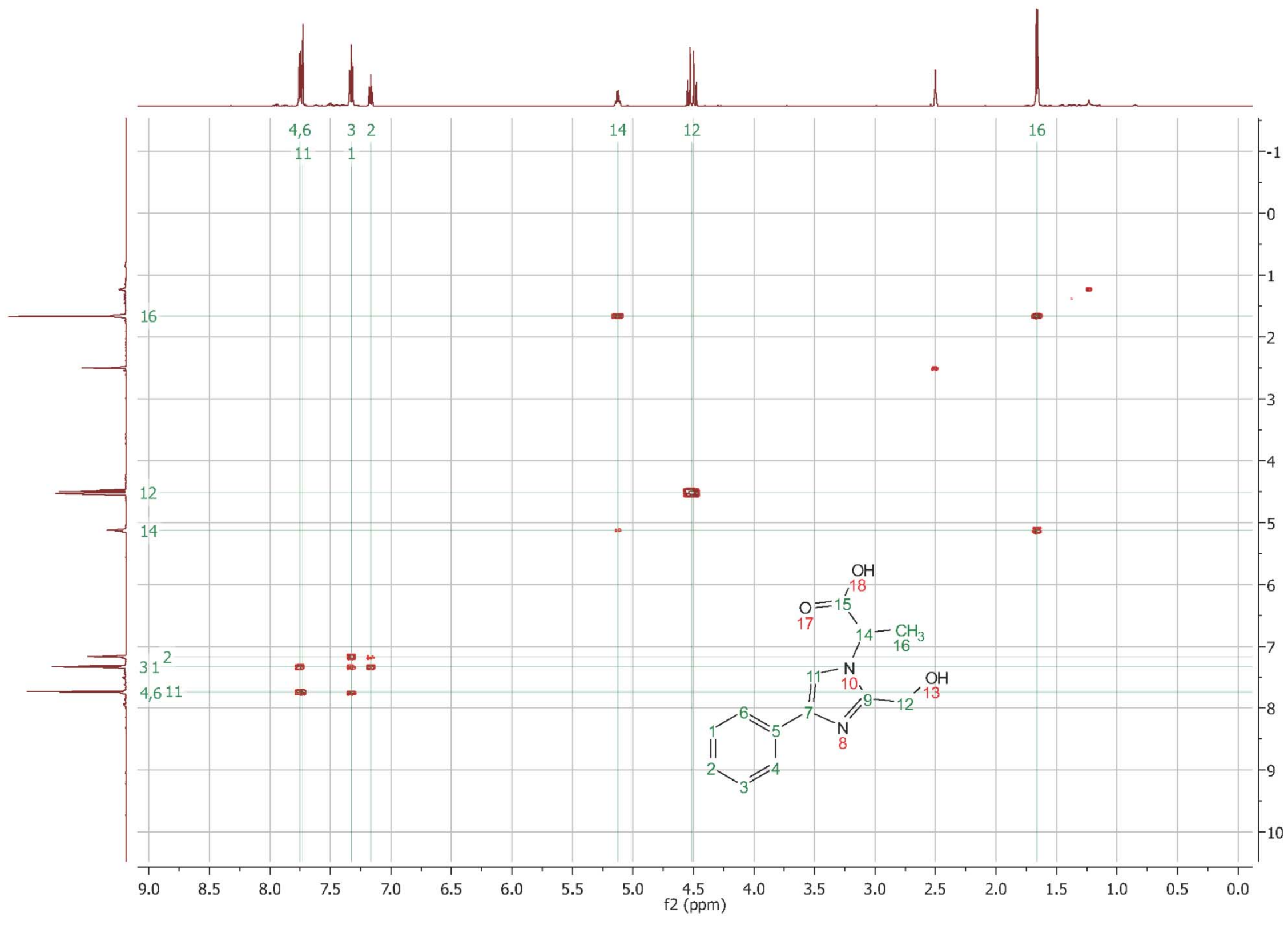


${ }^{1} \mathrm{H} /{ }^{13} \mathrm{C}$ HSQC NMR spectrum of $15 \mathrm{a}\left(600 / 150 \mathrm{MHz}\right.$, DMSO-d $\left.\mathrm{d}_{6}\right)$

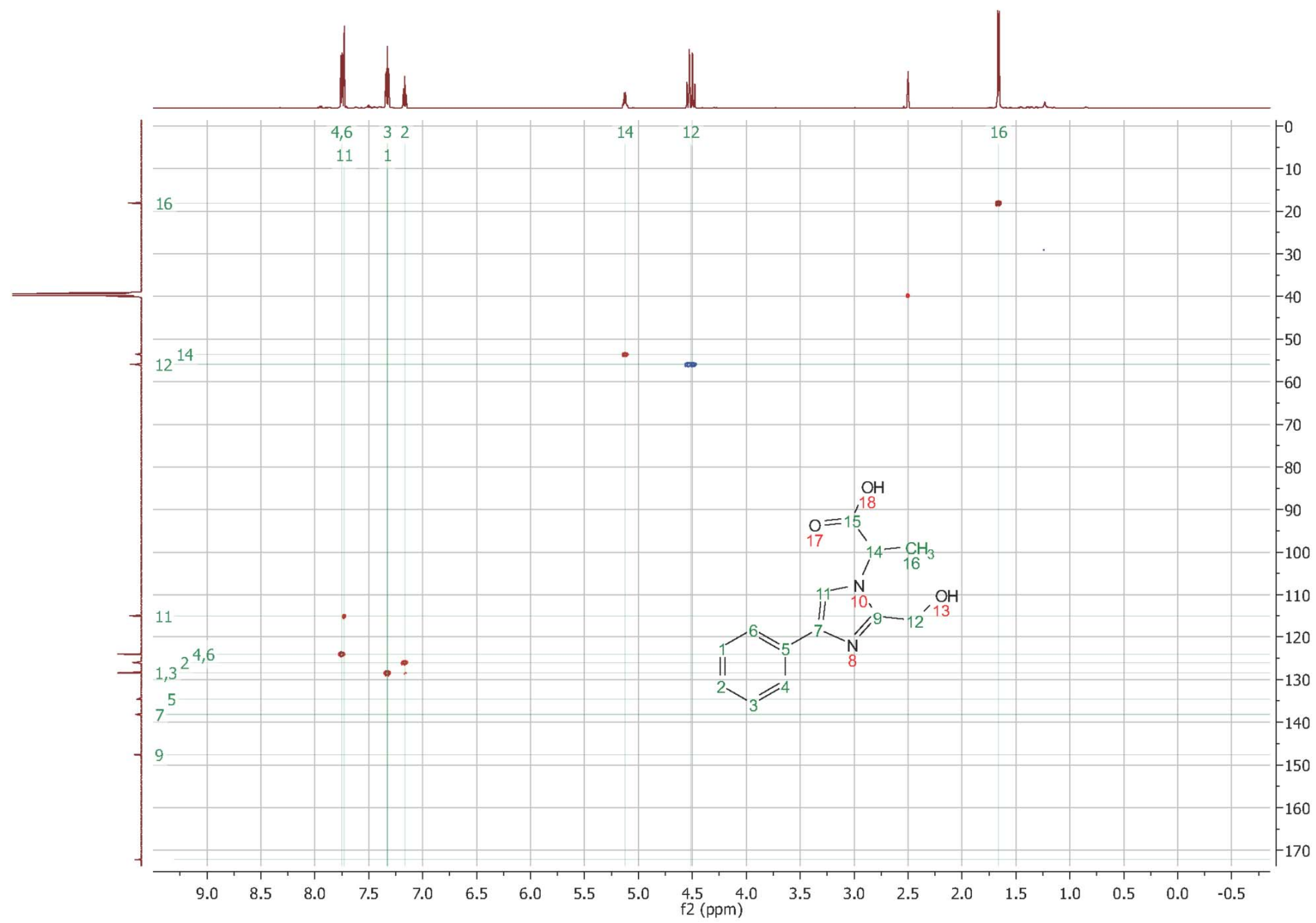


${ }^{1} \mathrm{H} /{ }^{13} \mathrm{C}$ HMBC NMR spectrum of $15 \mathrm{a}\left(600 / 150 \mathrm{MHz}\right.$, DMSO-d $\left.\mathrm{d}_{6}\right)$

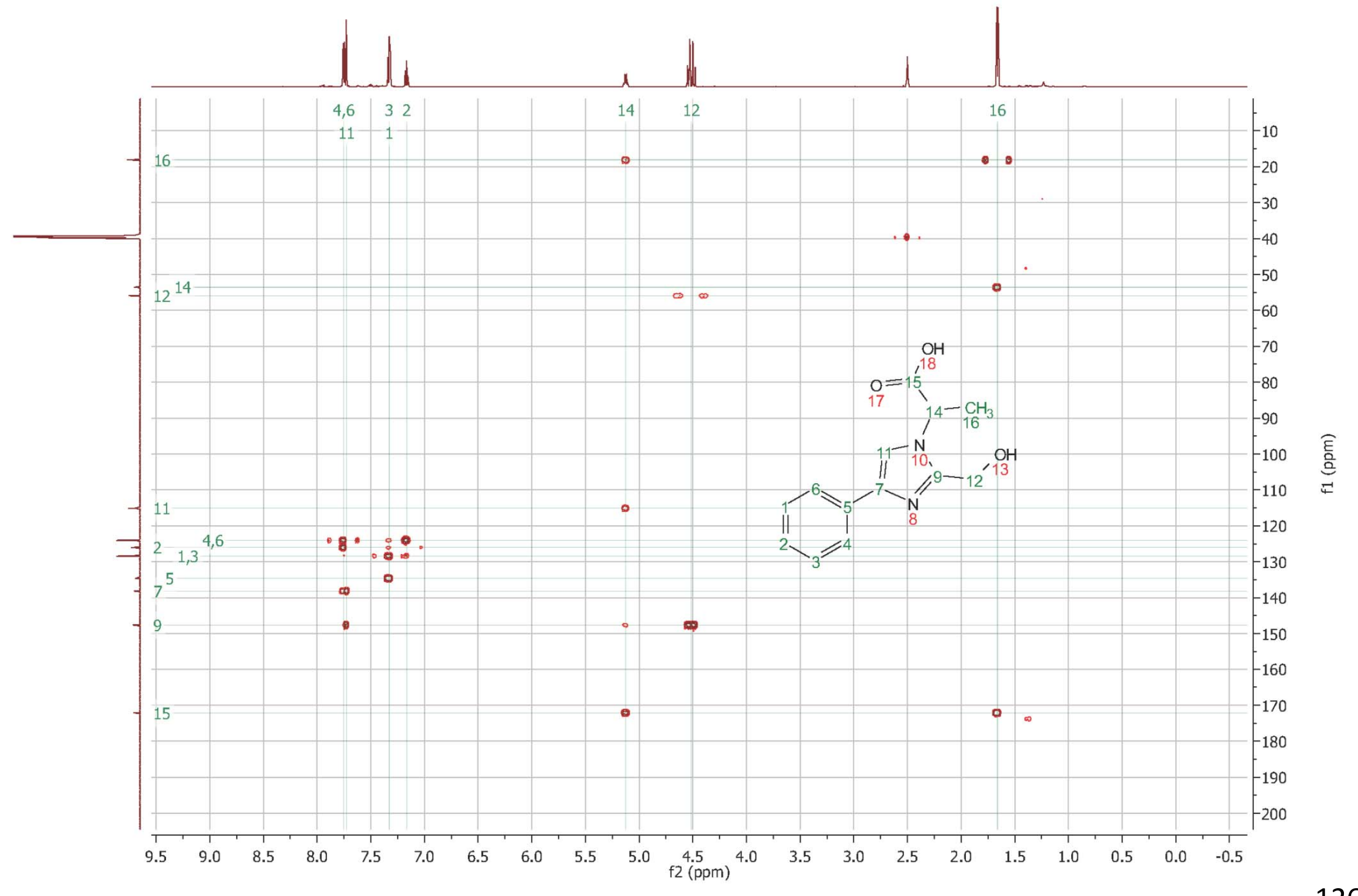


${ }^{1} \mathrm{H} /{ }^{1} \mathrm{H}$ ROESY NMR spectrum of $15 \mathrm{a}\left(600 / 600 \mathrm{MHz}\right.$, DMSO-d $\left.\mathrm{d}_{6}\right)$

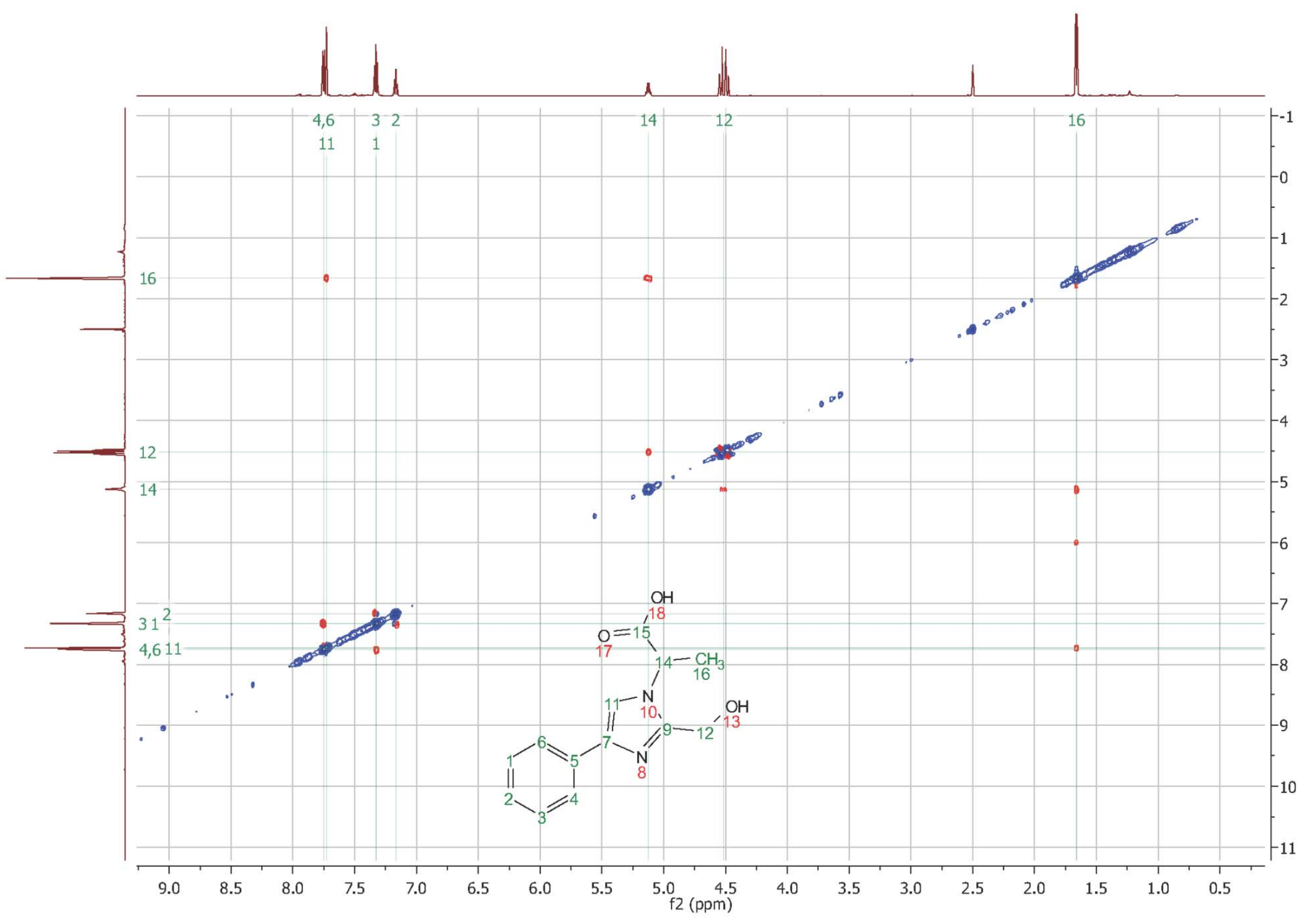


${ }^{1} \mathrm{H}$ NMR spectrum of $\mathbf{1 5 b}\left(600 \mathrm{MHz}, \mathrm{DMSO}-\mathrm{d}_{6}\right)$

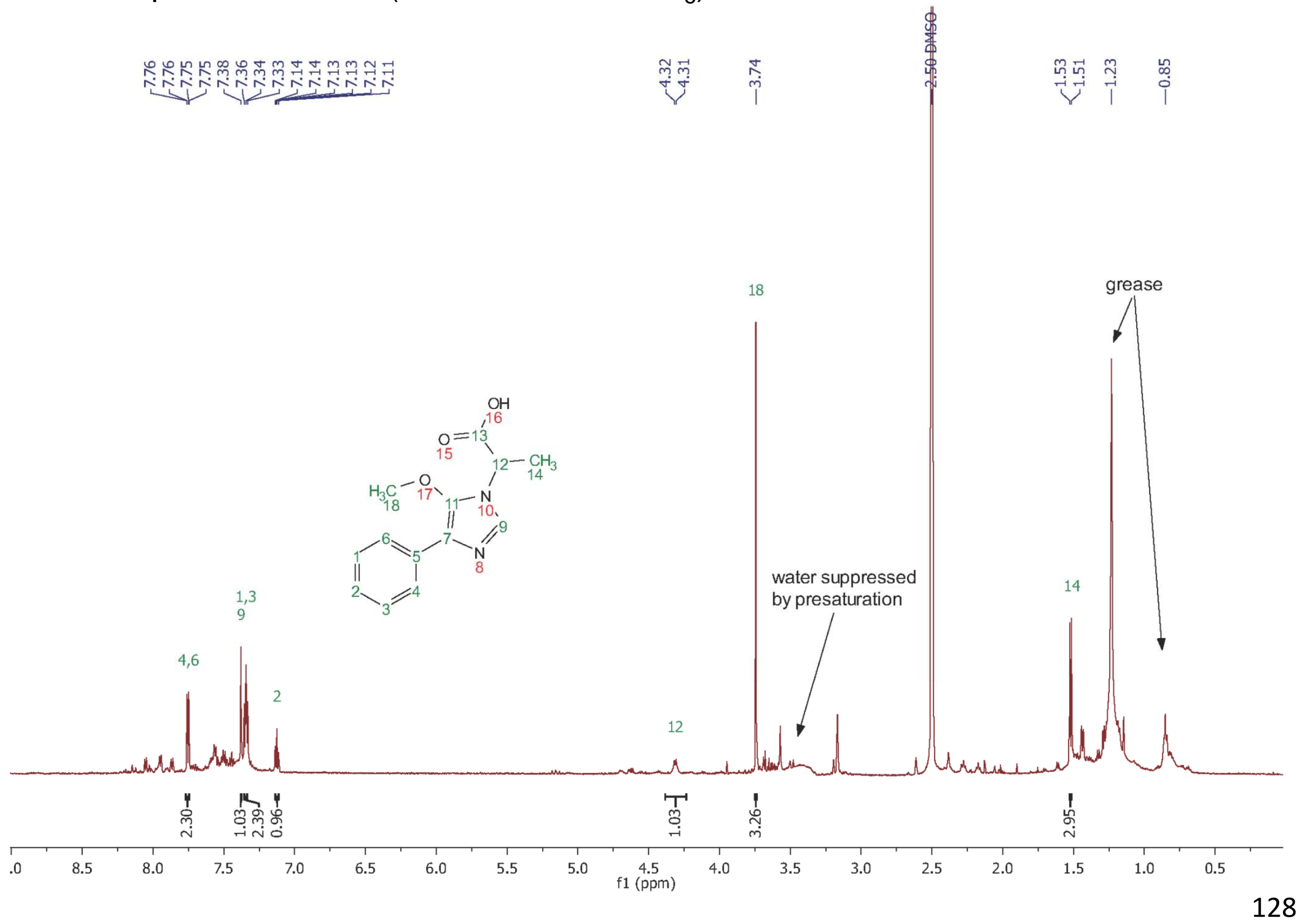


${ }^{1} \mathrm{H} /{ }^{1} \mathrm{H}$ COSY NMR spectrum of 15b (600 / $600 \mathrm{MHz}$, DMSO-d 6 )

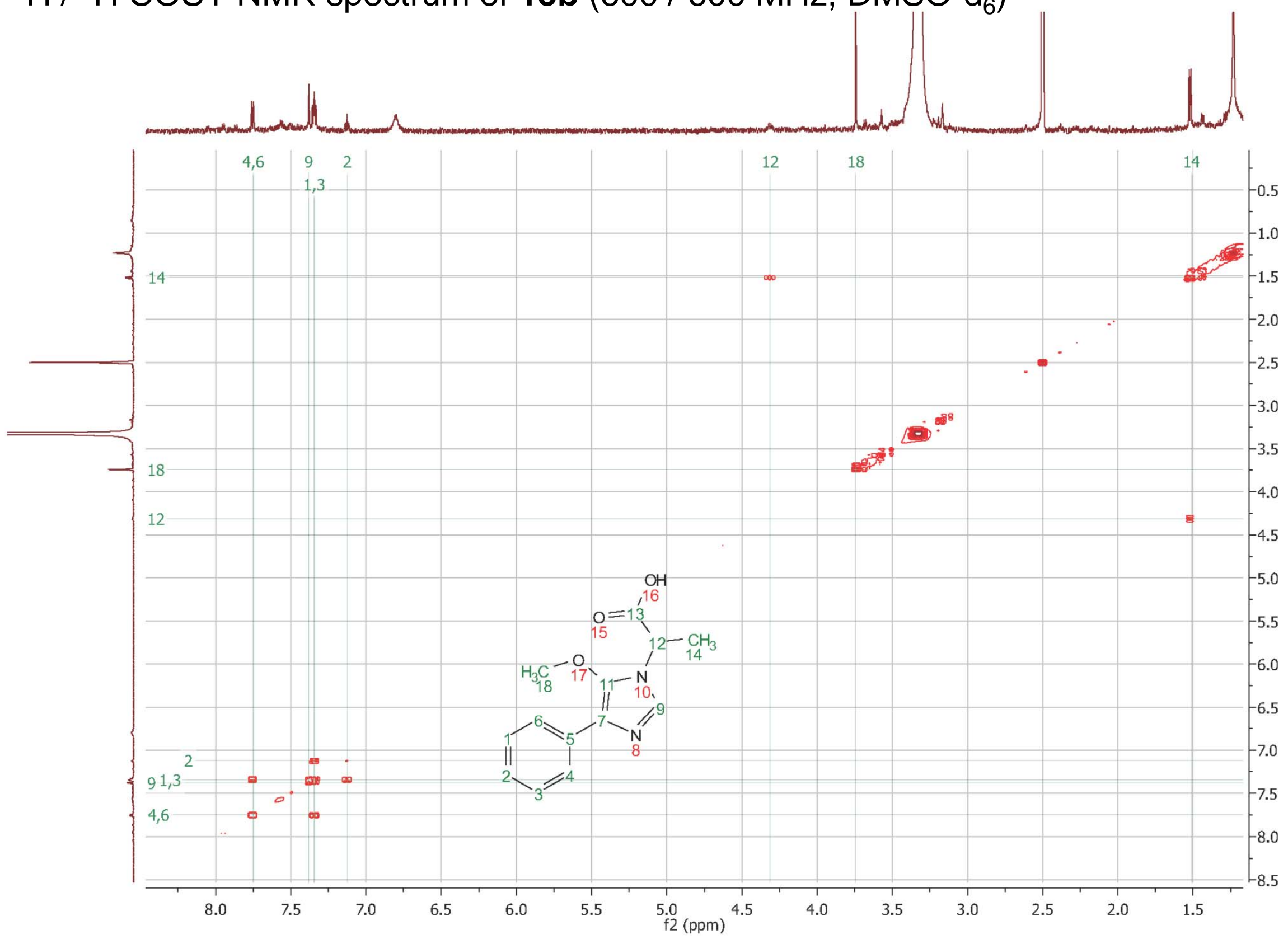


${ }^{1} \mathrm{H} /{ }^{13} \mathrm{C}$ HSQC NMR spectrum of 15b (600 / $150 \mathrm{MHz}$, DMSO-d 6 )

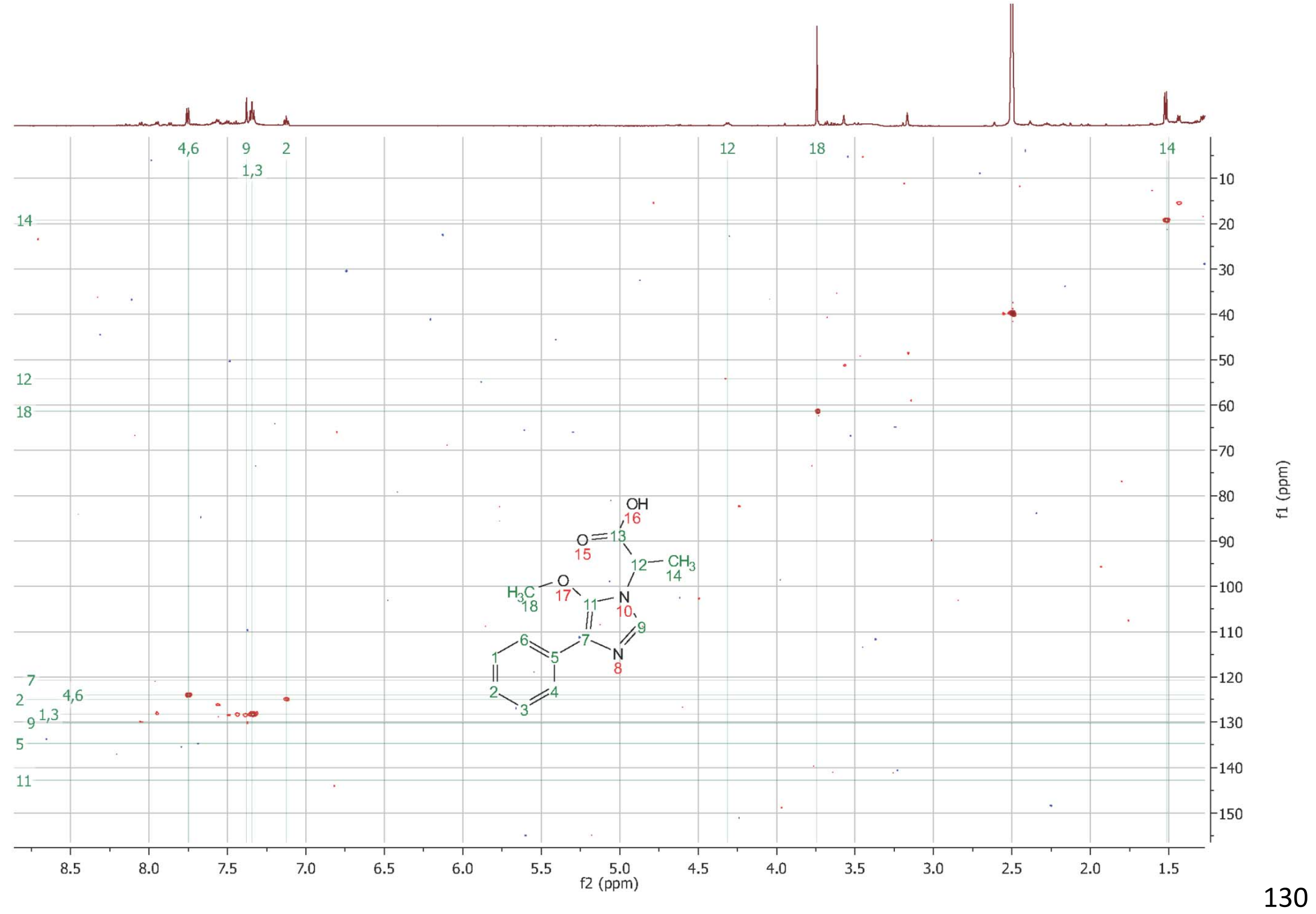


${ }^{1} \mathrm{H} /{ }^{13} \mathrm{C}$ HMBC NMR spectrum of 15b (600 / $150 \mathrm{MHz}$, DMSO-d 6 )

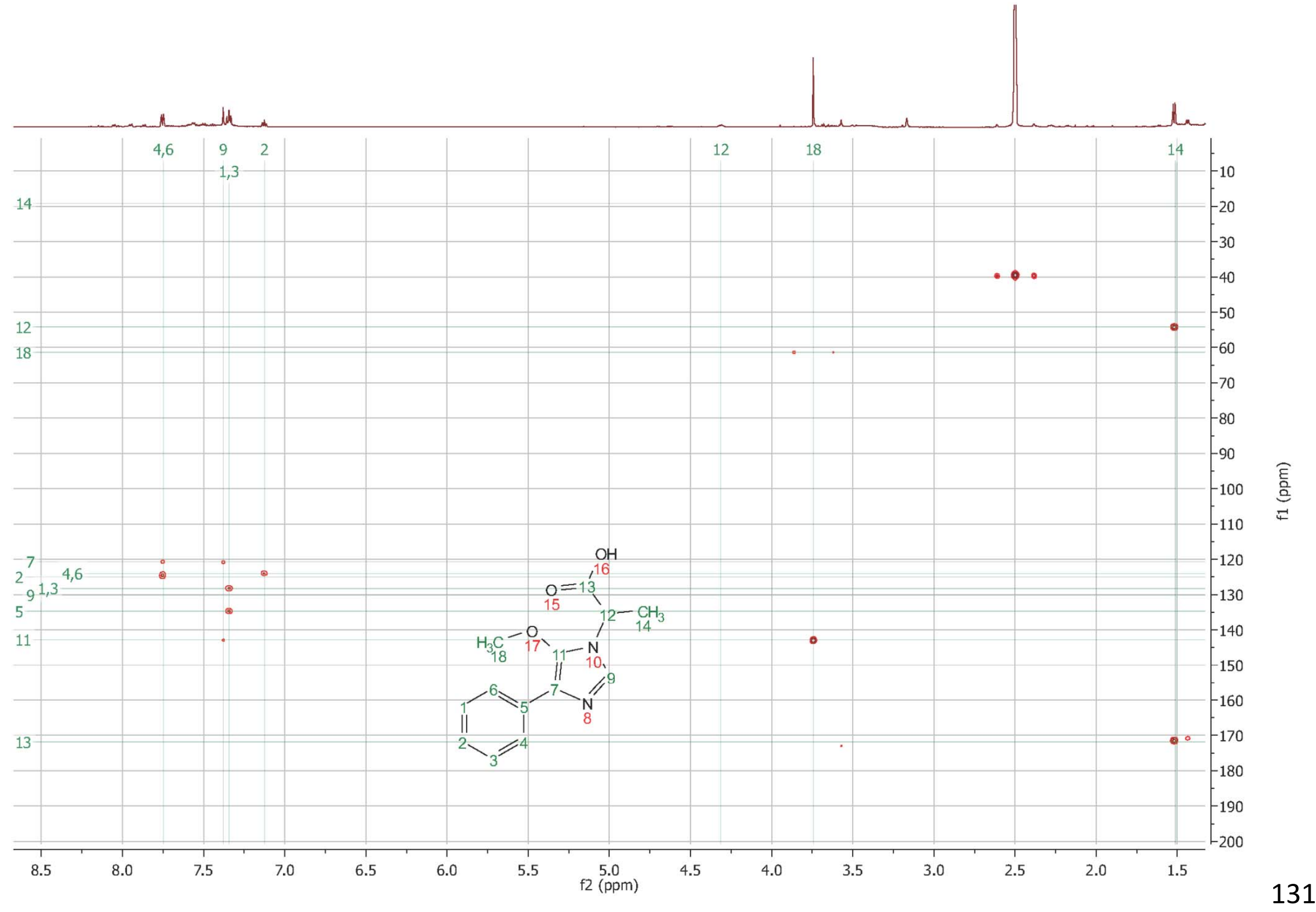


${ }^{1} \mathrm{H} /{ }^{1} \mathrm{H}$ ROESY NMR spectrum of $15 \mathbf{b}\left(600 / 600 \mathrm{MHz}\right.$, DMSO-d $\left.{ }_{6}\right)$

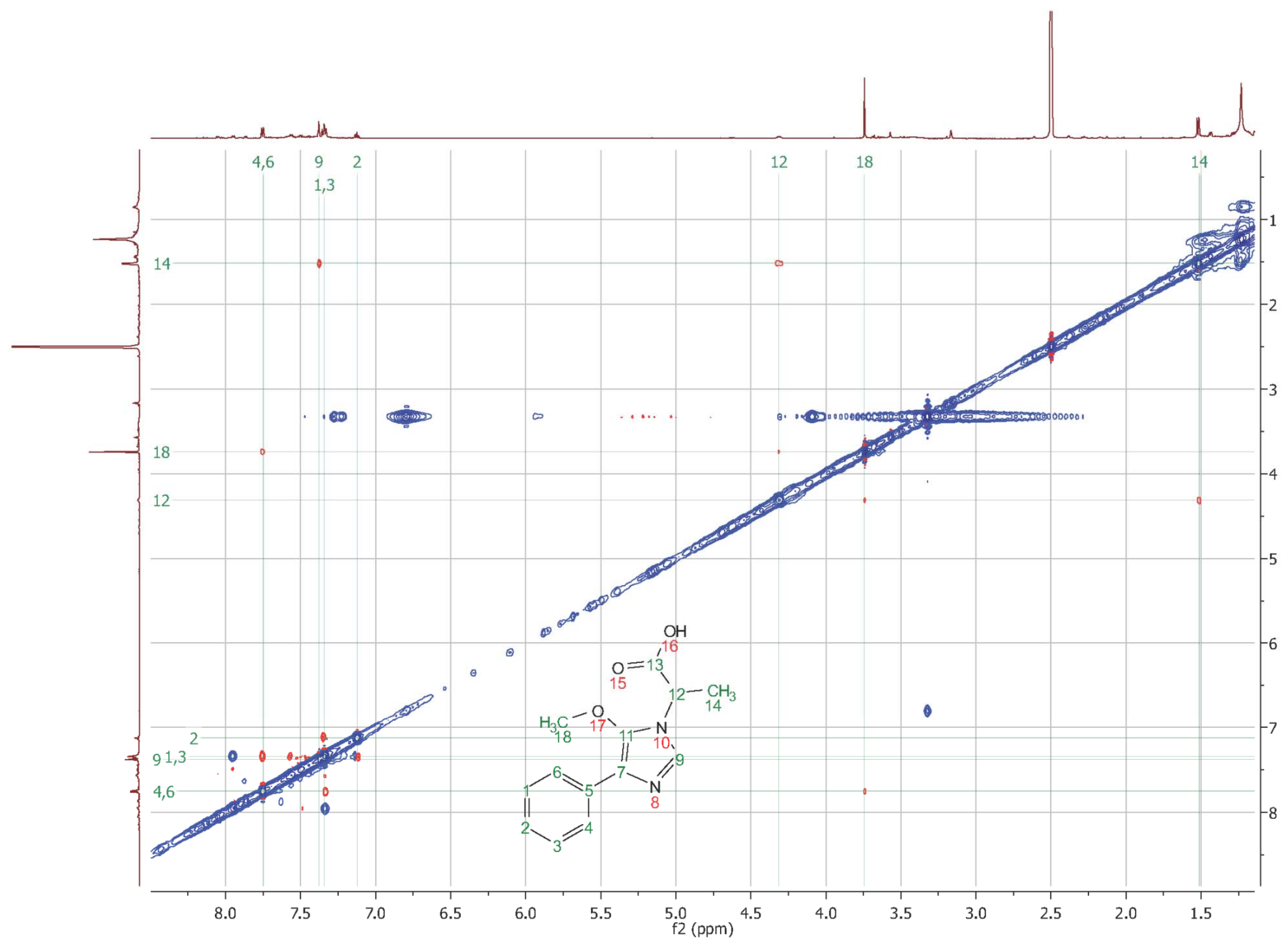


${ }^{1} \mathrm{H}$ NMR spectrum of $16 \mathbf{a}\left(500 \mathrm{MHz}, \mathrm{CD}_{3} \mathrm{OD}\right)$

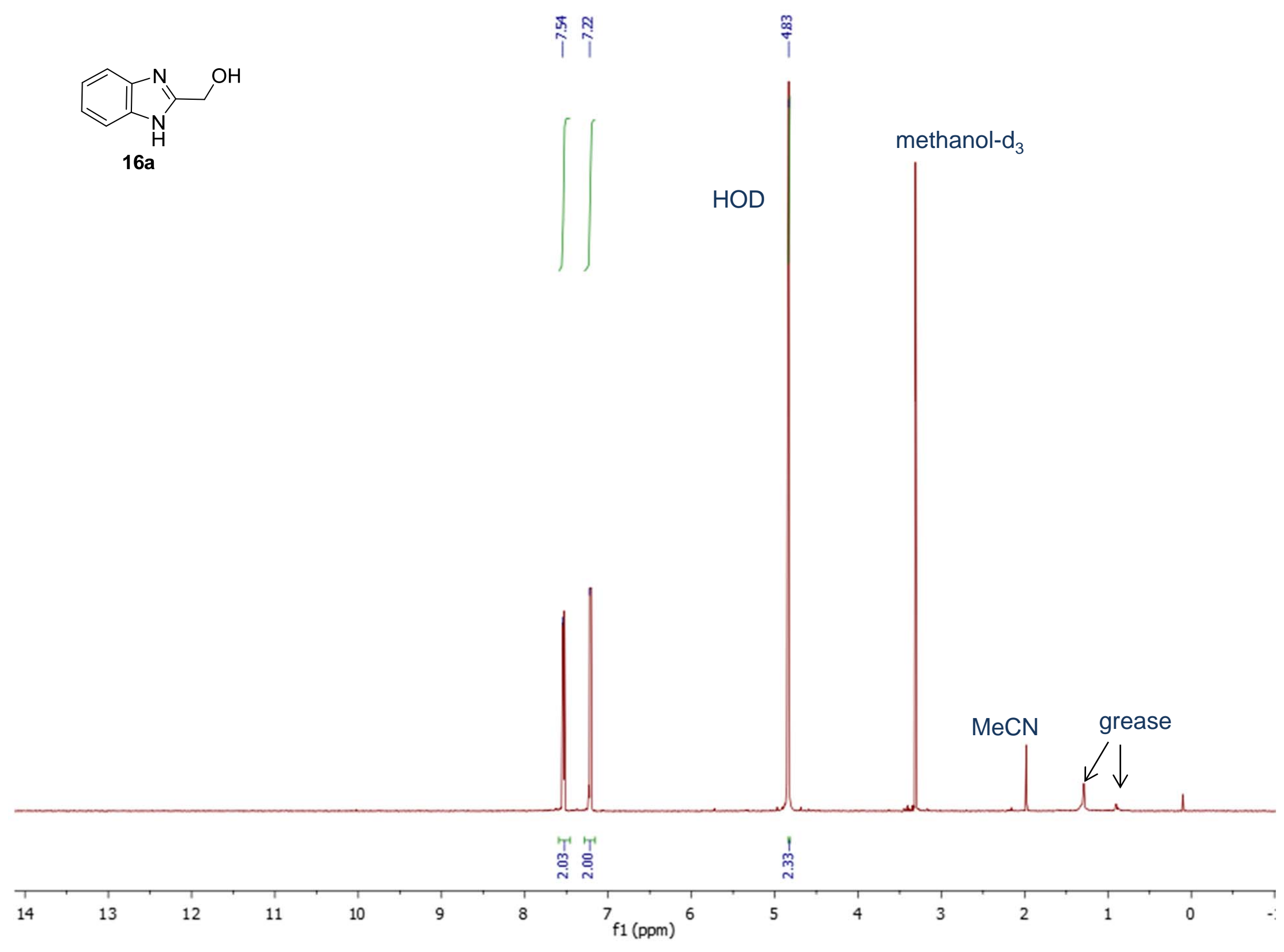


${ }^{13} \mathrm{C}$ NMR spectrum of $16 \mathbf{a}\left(125 \mathrm{MHz}, \mathrm{CD}_{3} \mathrm{OD}\right)$

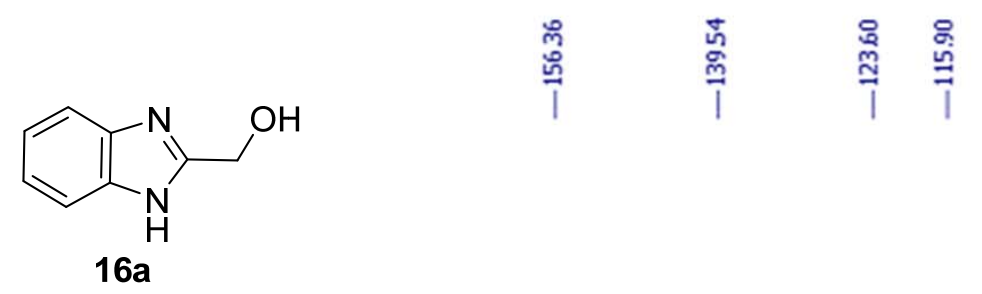

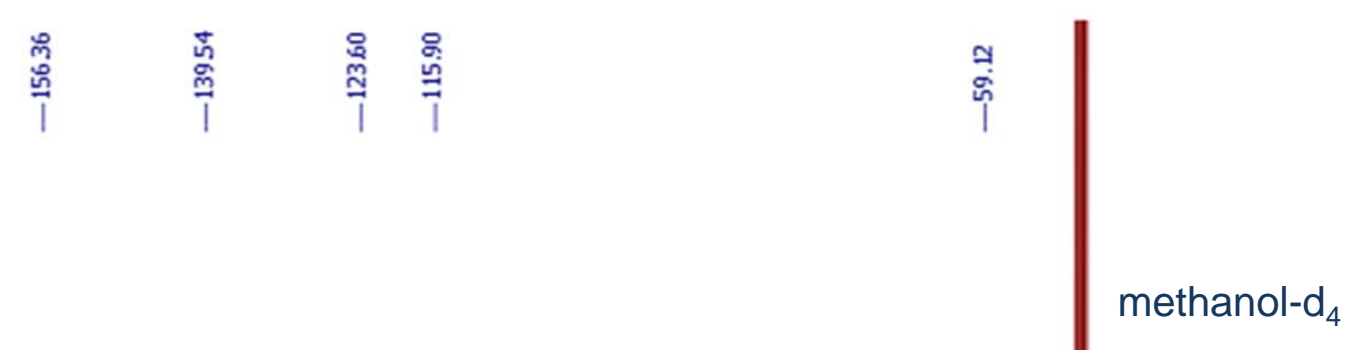

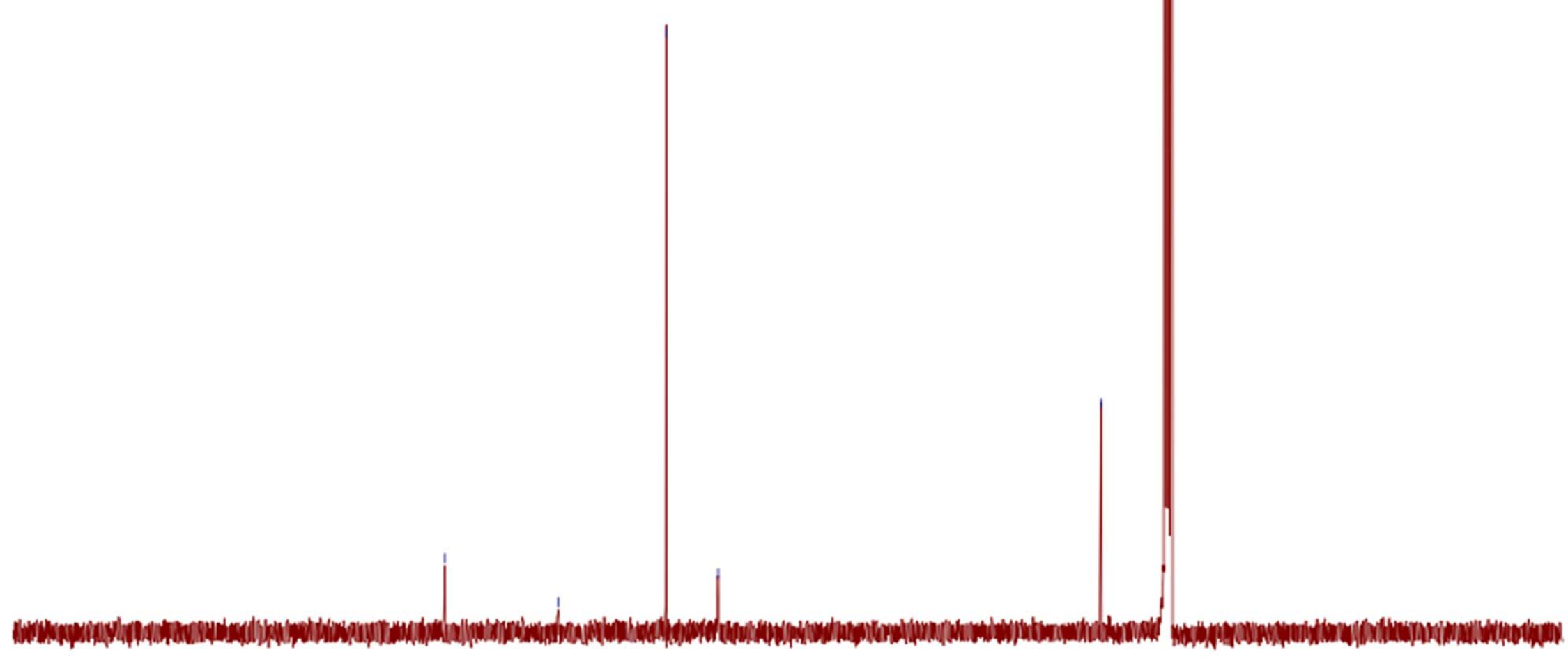

$20 \quad 210 \quad 200$

190

180

170

150

140

130

120

$110 \quad 100$
$\mathrm{f1}(\mathrm{ppm})$

90

80

60

50

30

20

S134 
${ }^{1} \mathrm{H}$ NMR spectrum of $17 \mathrm{a}\left(500 \mathrm{MHz}, \mathrm{DMSO}-\mathrm{d}_{6}\right)$

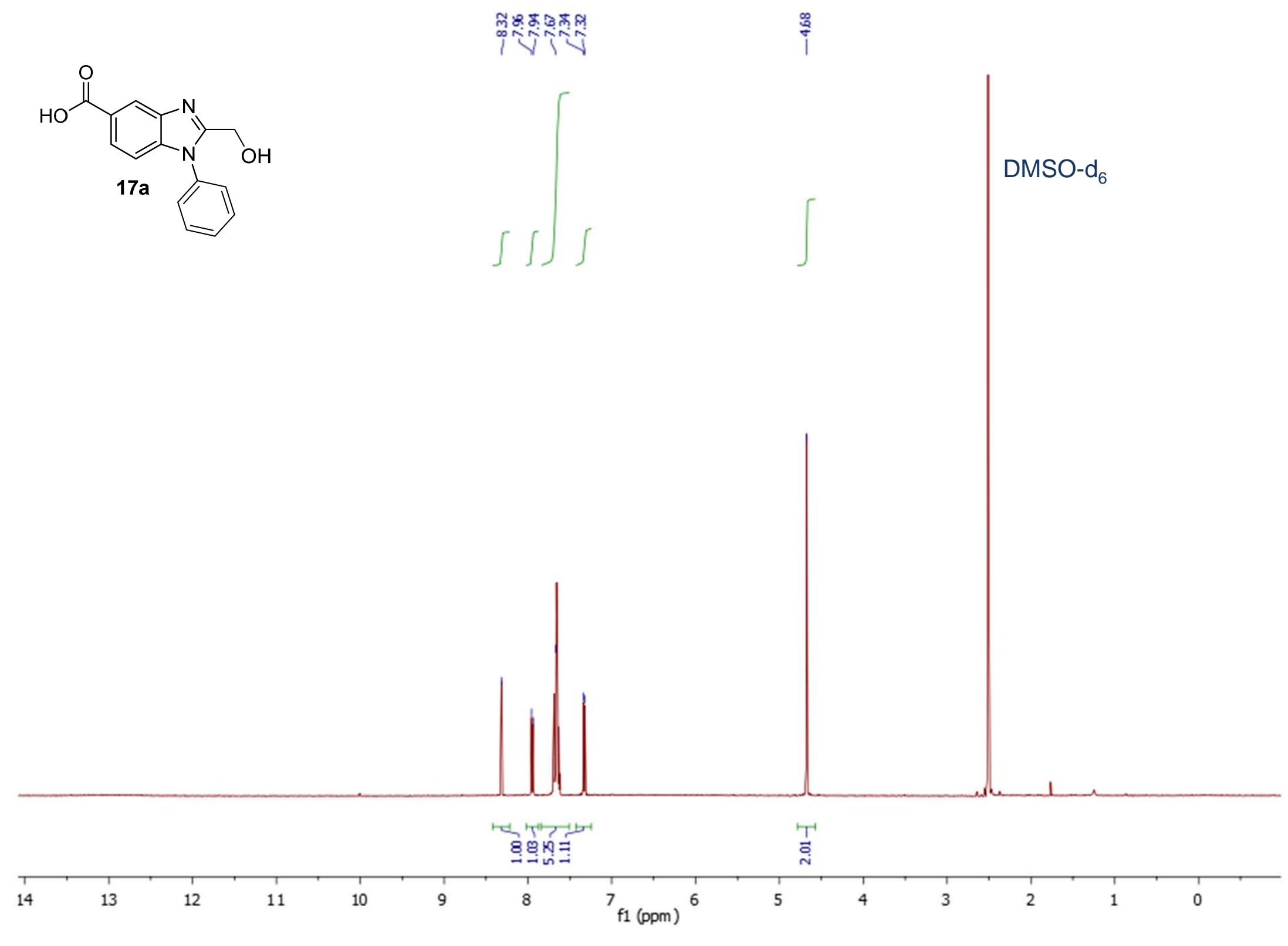


${ }^{13} \mathrm{C}$ NMR spectrum of $17 \mathrm{a}\left(125 \mathrm{MHz}\right.$, DMSO-d $\left.\mathrm{d}_{6}\right)$

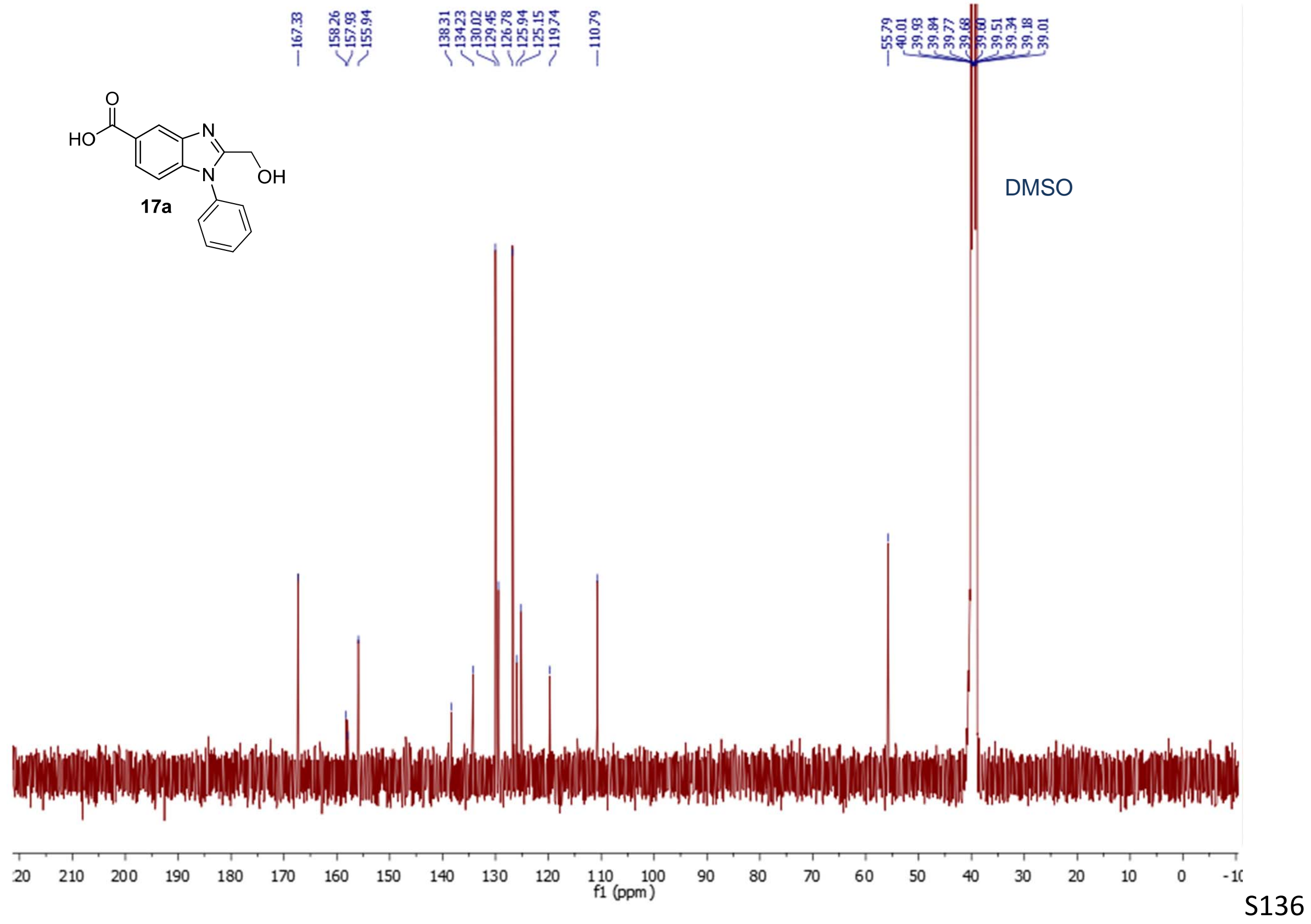


${ }^{1} \mathrm{H}$ NMR spectrum of $18 \mathrm{a}\left(500 \mathrm{MHz}, \mathrm{CD}_{3} \mathrm{OD}\right)$

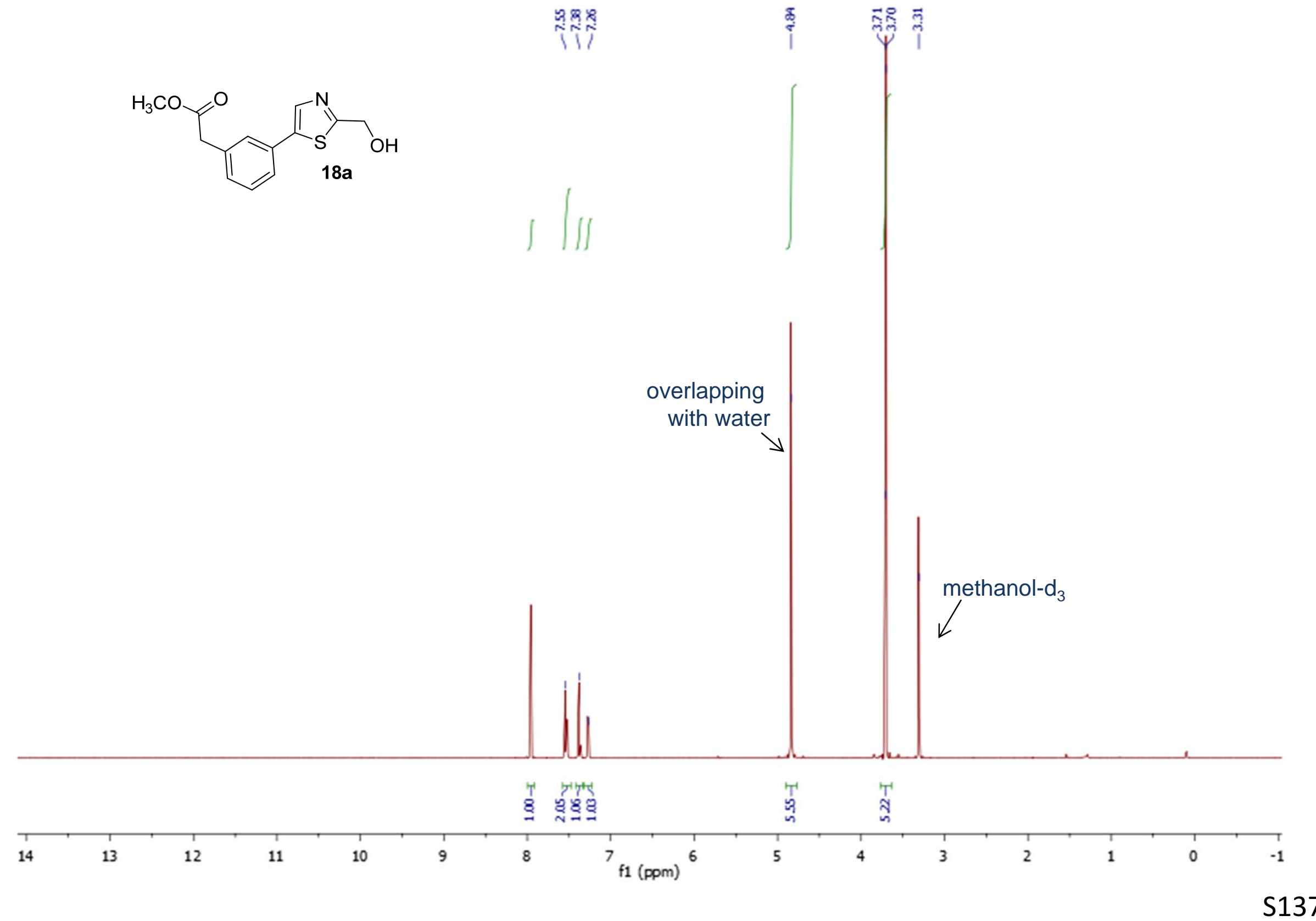


${ }^{13} \mathrm{C}$ NMR spectrum of $18 \mathbf{a}\left(125 \mathrm{MHz}, \mathrm{CD}_{3} \mathrm{OD}\right)$
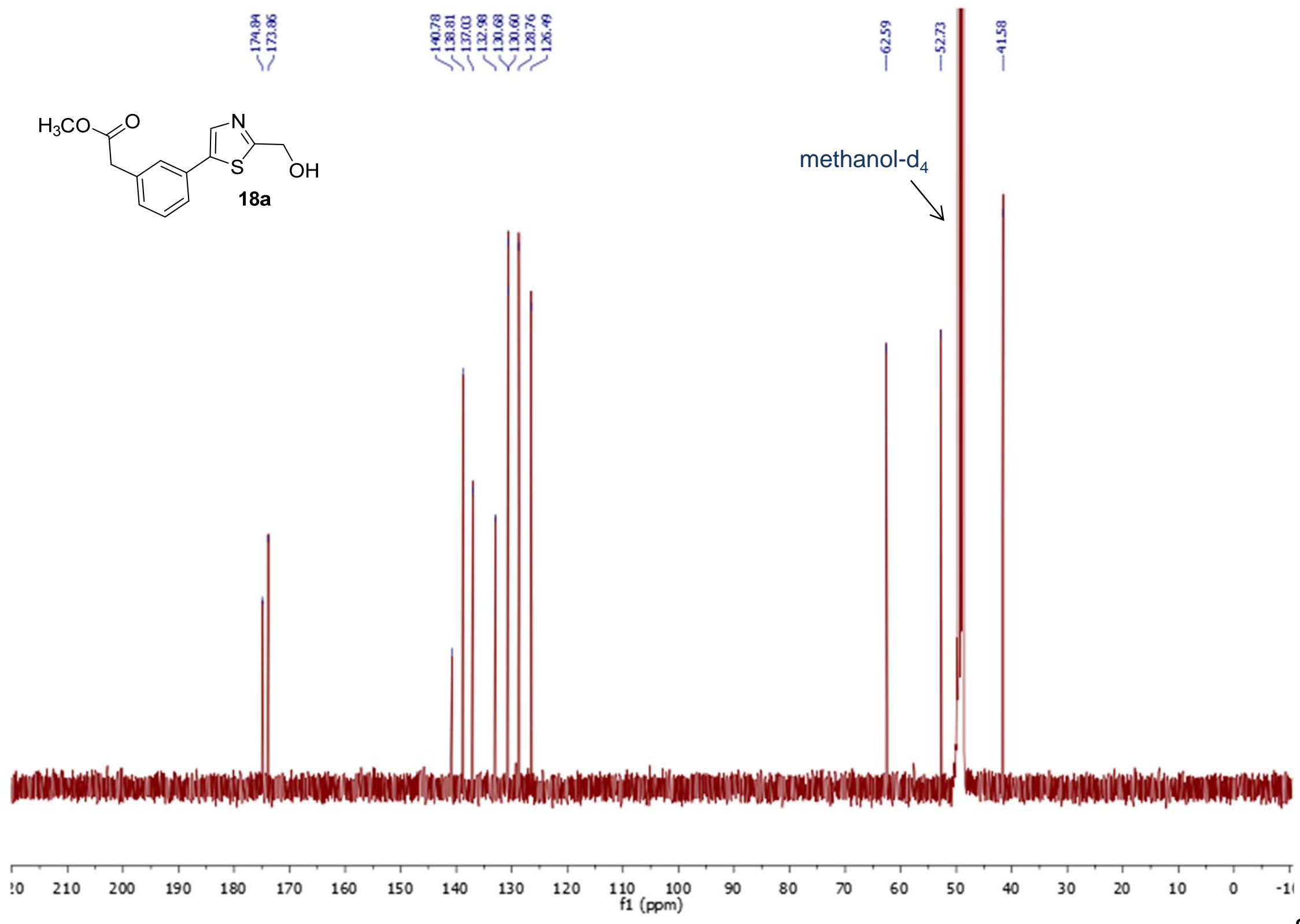
${ }^{1} \mathrm{H}$ NMR spectrum of $19 a\left(500 \mathrm{MHz}, \mathrm{DMSO}-\mathrm{d}_{6}\right)$
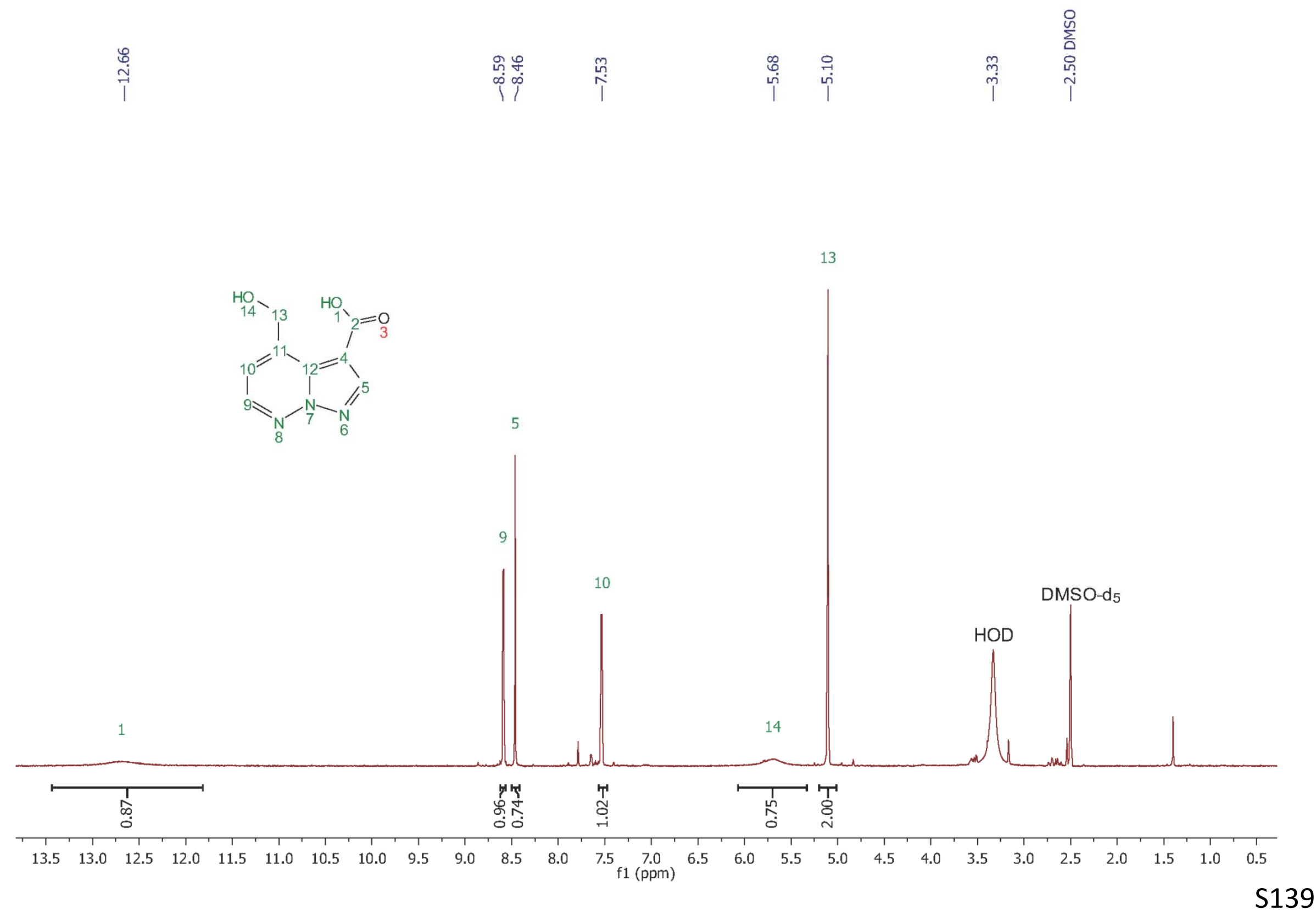
${ }^{13} \mathrm{C}$ NMR spectrum of $19 a\left(125 \mathrm{MHz}\right.$, DMSO-d $\left.\mathrm{d}_{6}\right)$

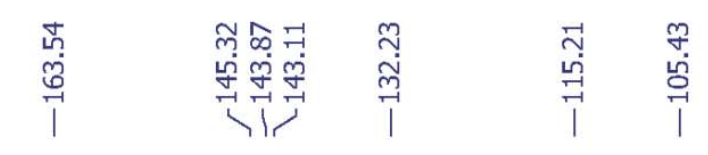
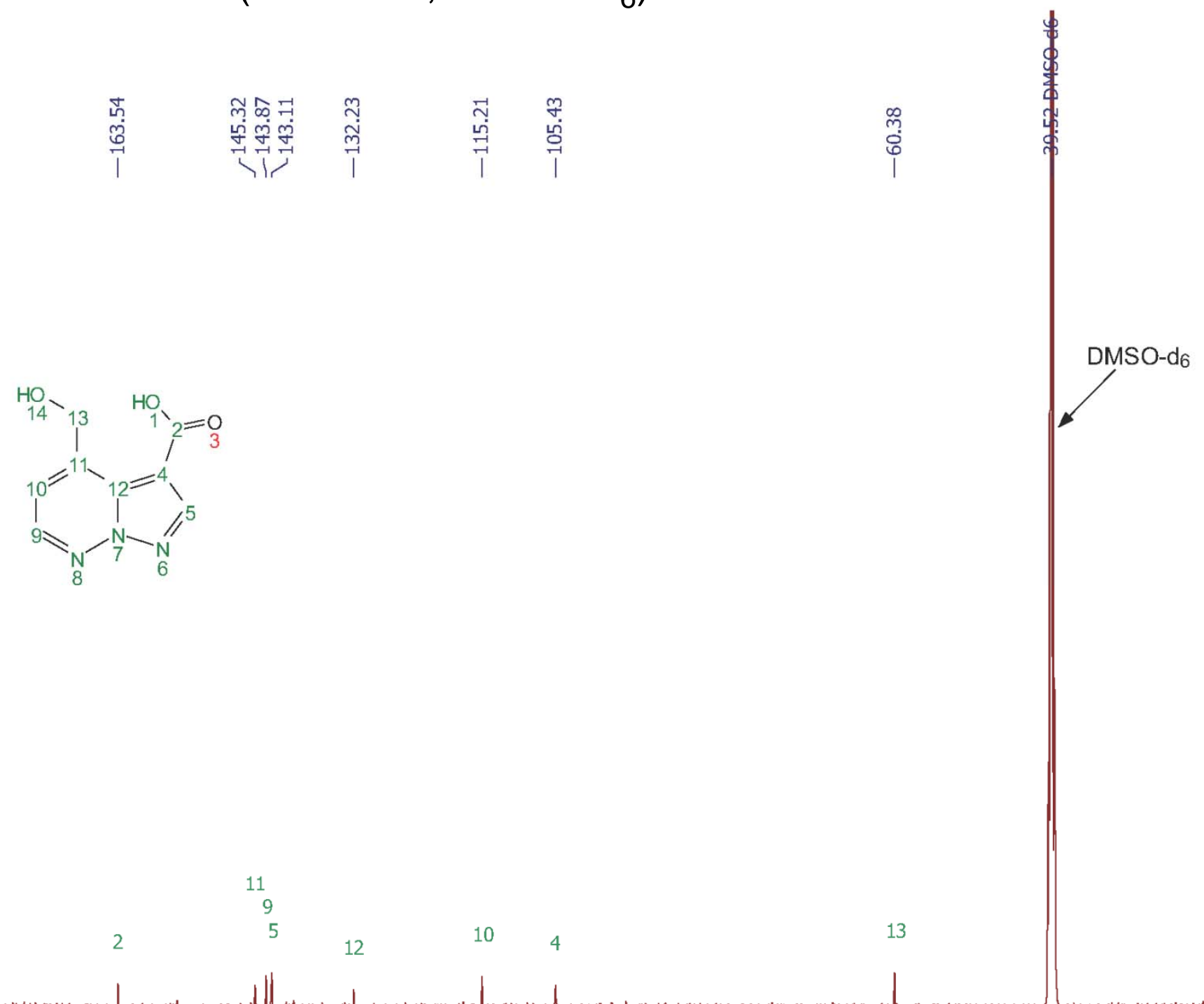

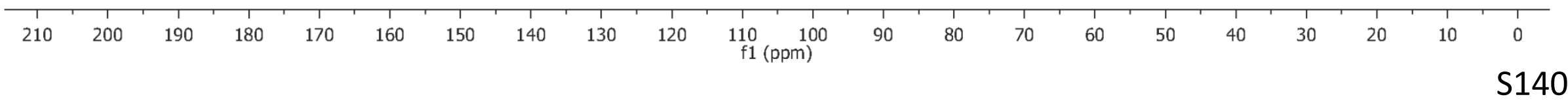


${ }^{1} \mathrm{H} /{ }^{1} \mathrm{H}$ COSY NMR spectrum of $19 a(500 / 500 \mathrm{MHz}$, DMSO-d 6 )

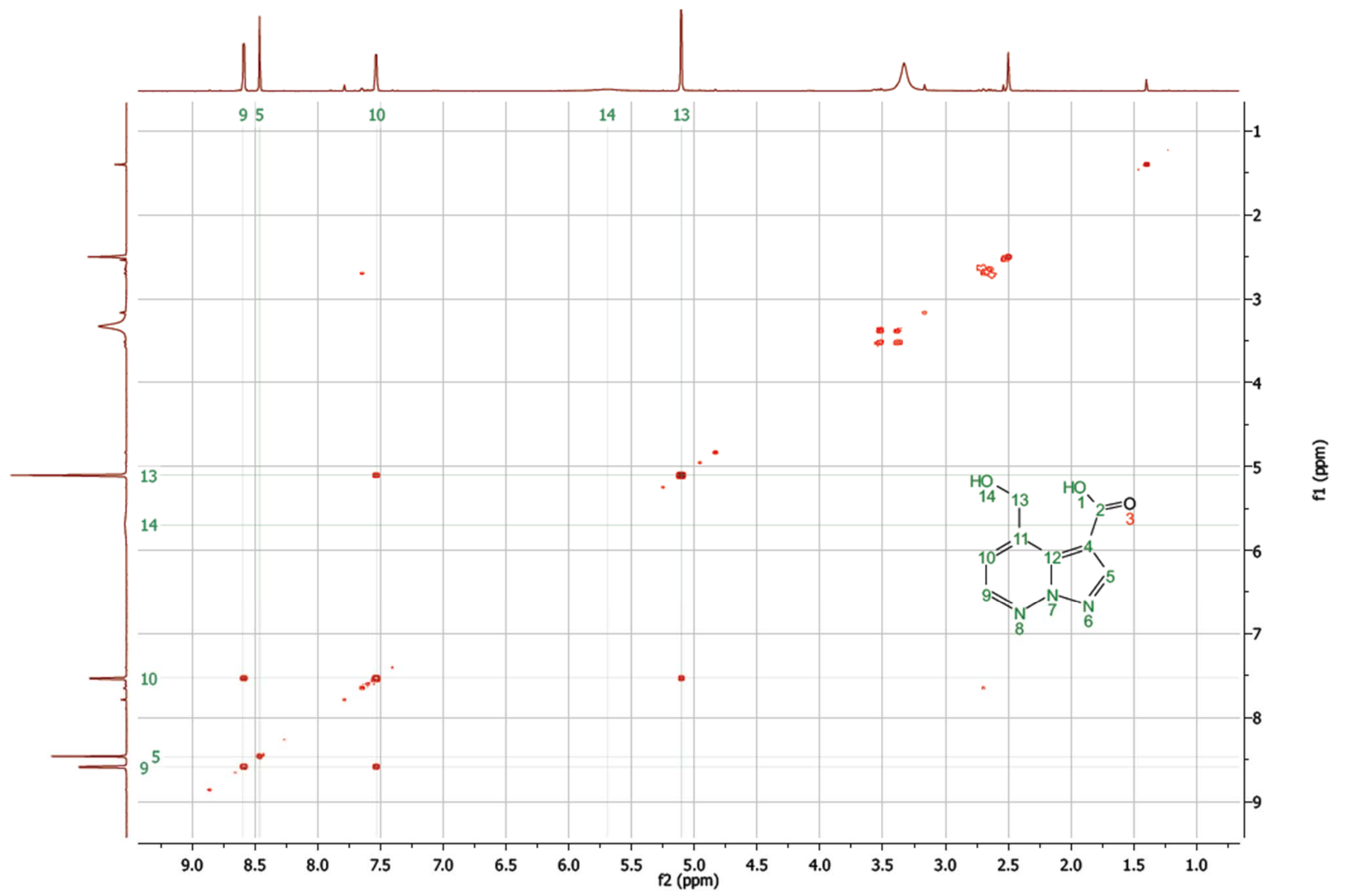


${ }^{1} \mathrm{H} /{ }^{13} \mathrm{C}$ HSQC NMR spectrum of $19 a\left(500 / 125 \mathrm{MHz}\right.$, DMSO-d $\left.\mathrm{d}_{6}\right)$

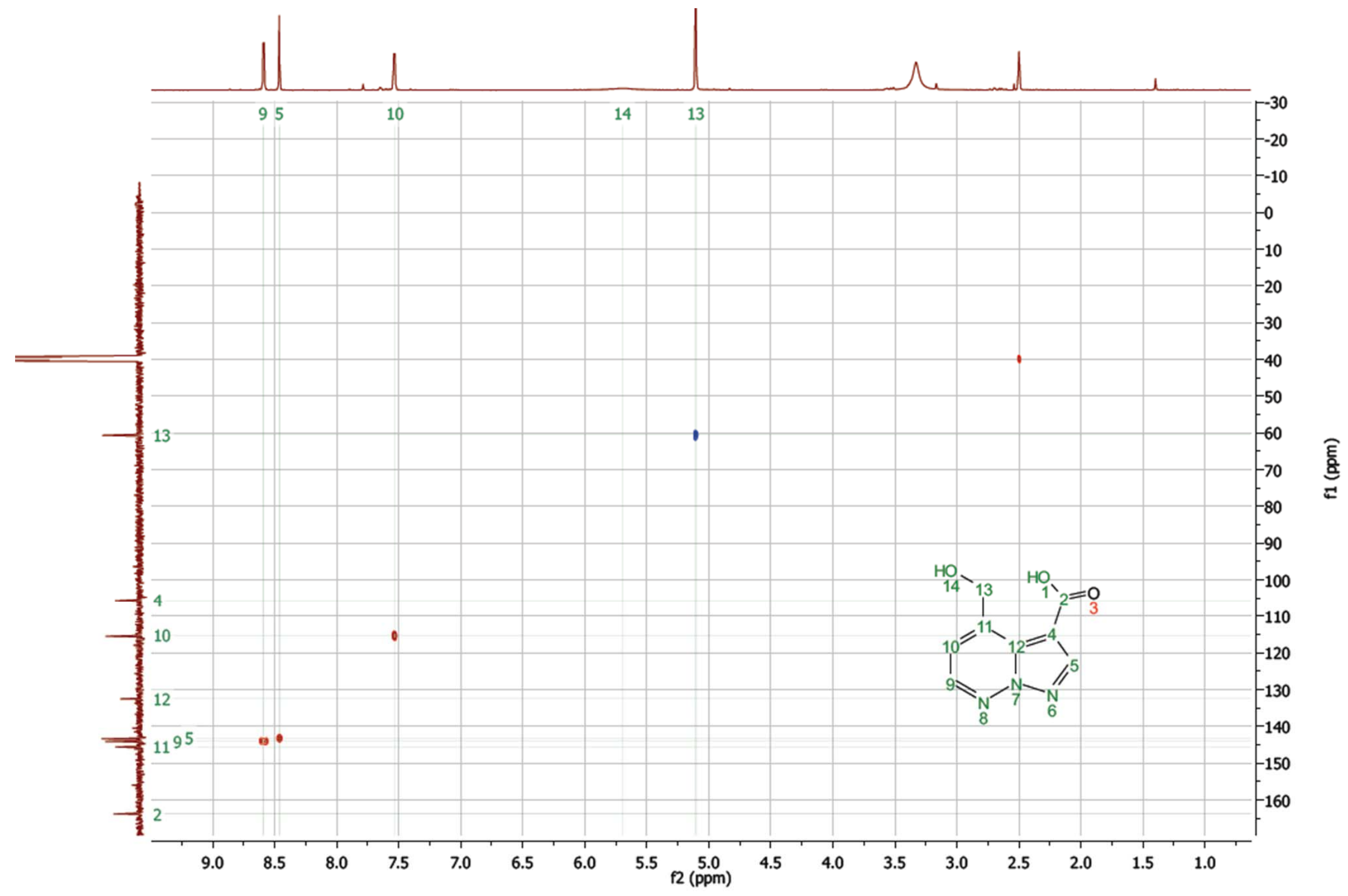


${ }^{1} \mathrm{H} /{ }^{13} \mathrm{C}$ HMBC NMR spectrum of $19 a\left(500 / 125 \mathrm{MHz}\right.$, DMSO-d $\left.{ }_{6}\right)$

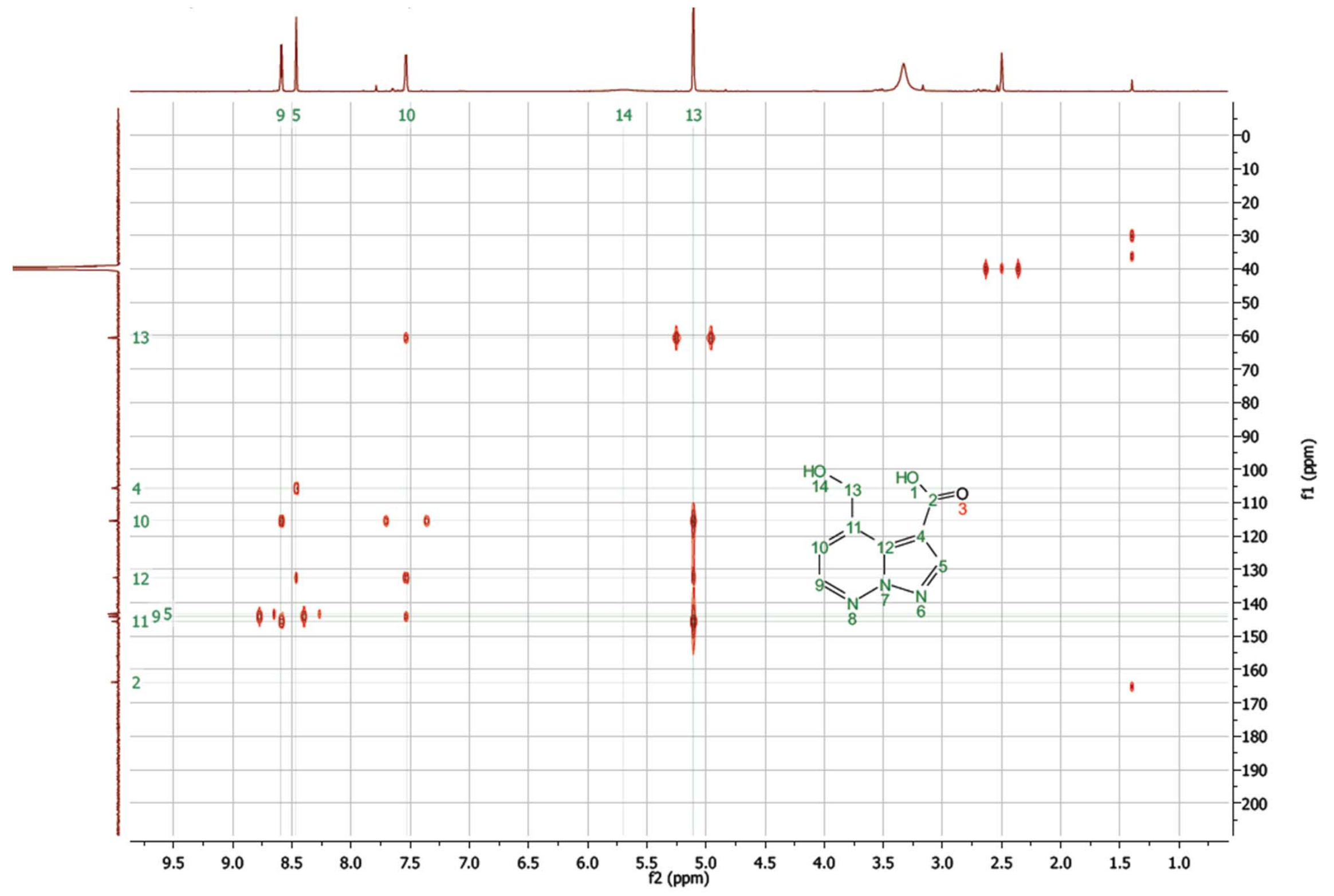


${ }^{1} \mathrm{H} /{ }^{1} \mathrm{H}$ ROESY NMR spectrum of $19 \mathrm{a}\left(500 / 500 \mathrm{MHz}\right.$, DMSO-d $\left.\mathrm{d}_{6}\right)$

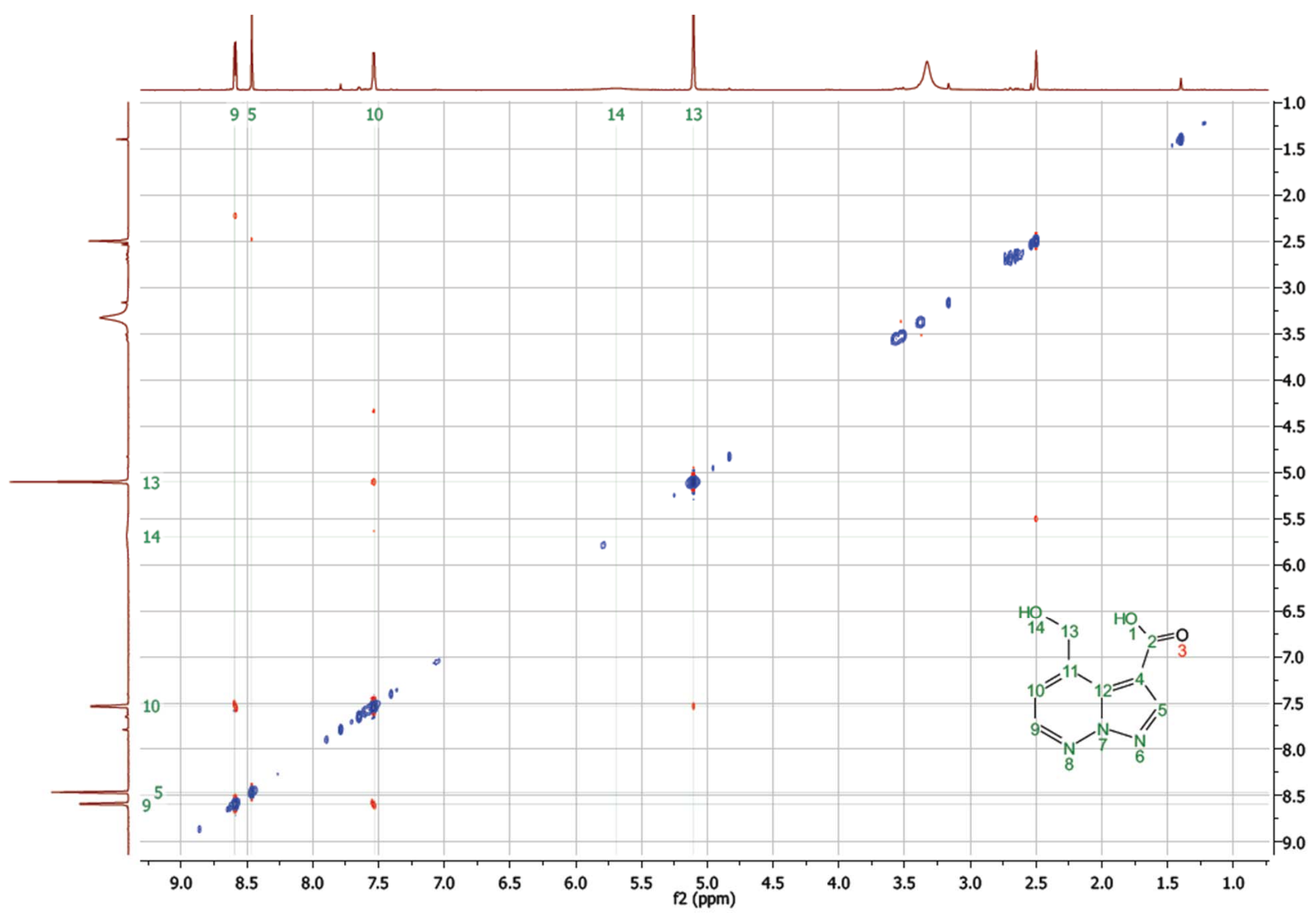


${ }^{1} \mathrm{H} /{ }^{15} \mathrm{~N}$ HMBC NMR spectrum of $19 \mathrm{a}\left(500 / 51 \mathrm{MHz}, \mathrm{CD}_{3} \mathrm{OD}\right)$

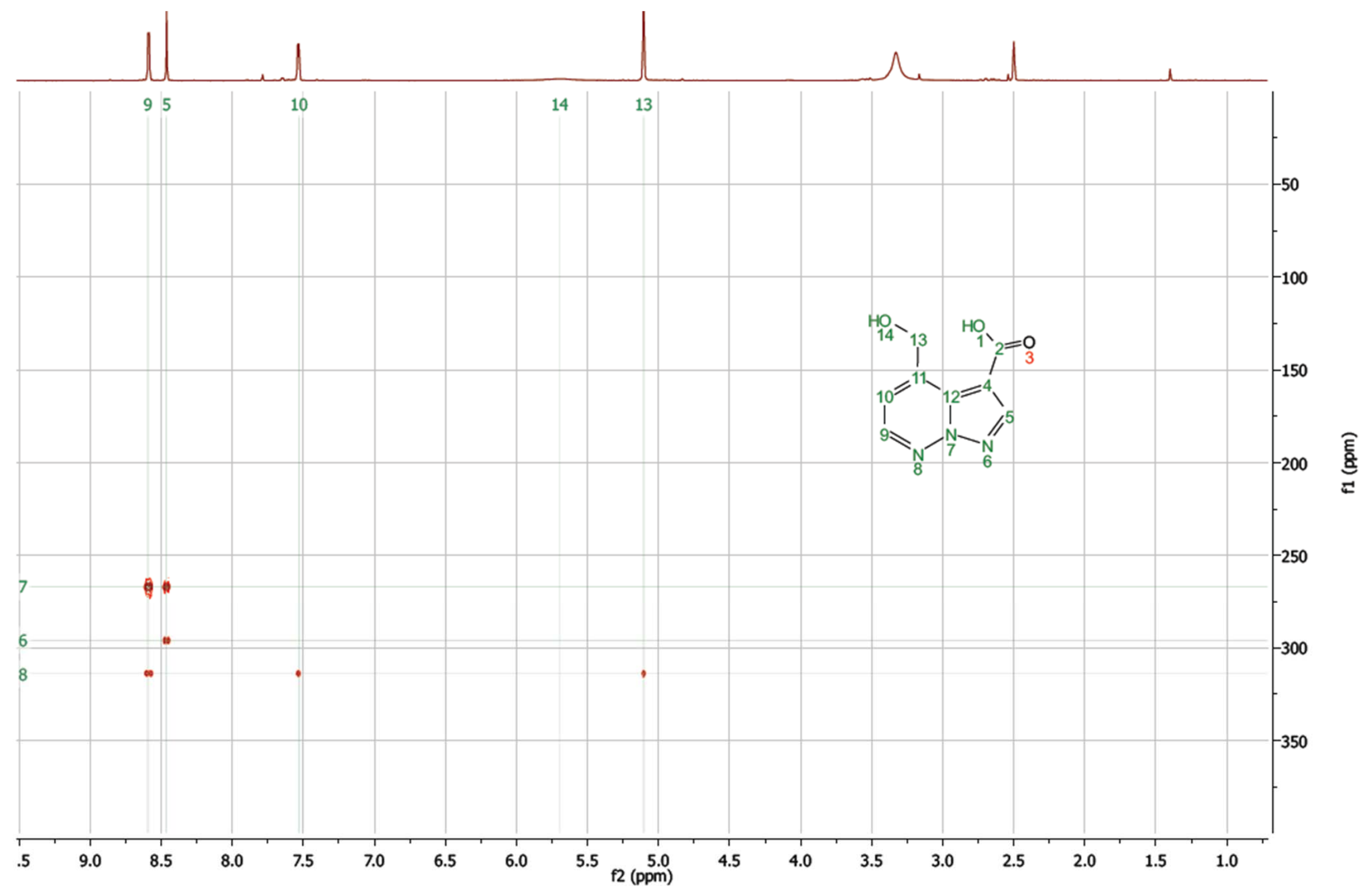




\section{${ }^{1} \mathrm{H}$ NMR spectrum of $20 \mathrm{a}\left(500 \mathrm{MHz}, \mathrm{CD}_{3} \mathrm{OD}\right)$}

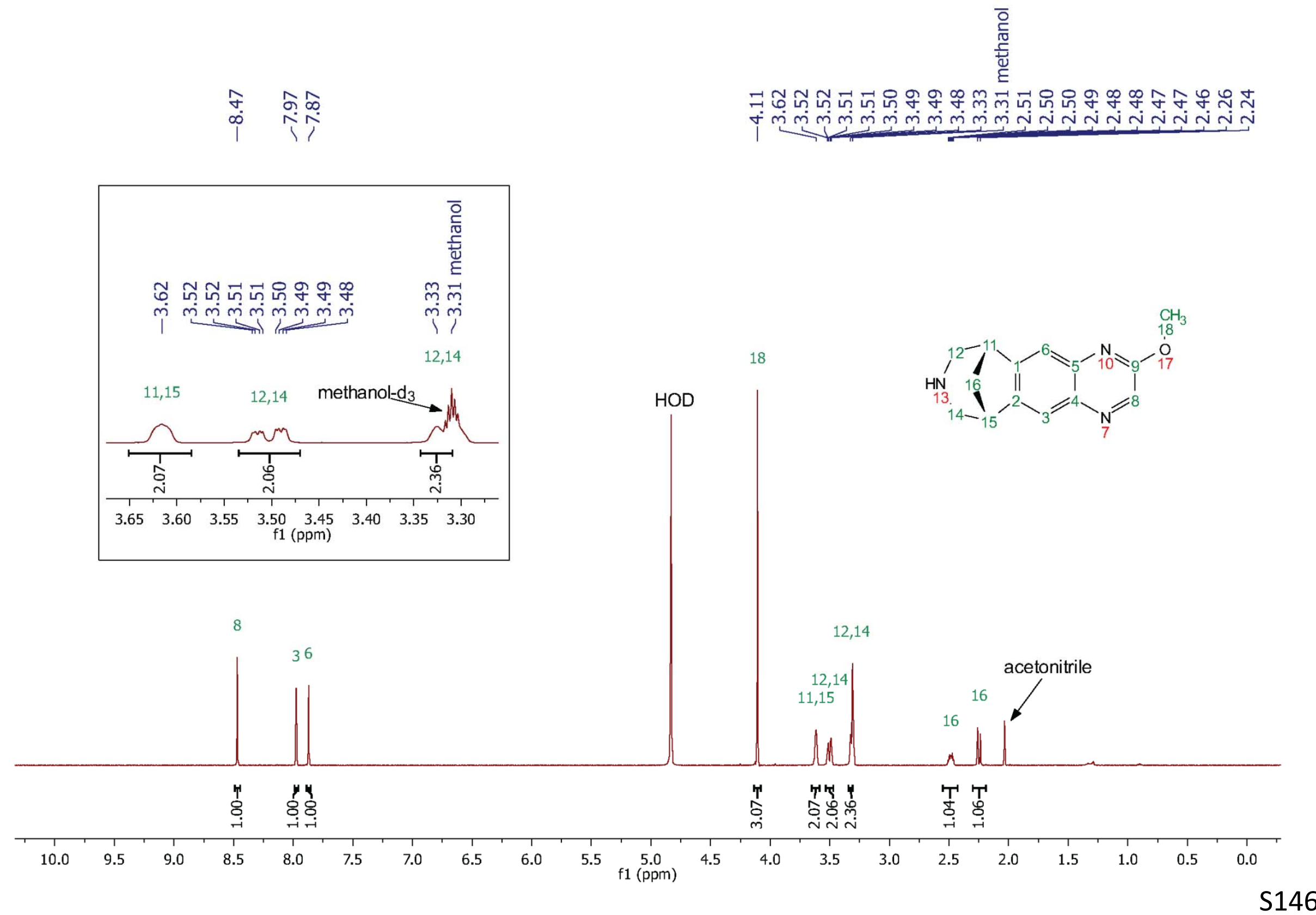


${ }^{13} \mathrm{C}$ NMR spectrum of $20 \mathrm{a}\left(125 \mathrm{MHz}, \mathrm{CD}_{3} \mathrm{OD}\right)$
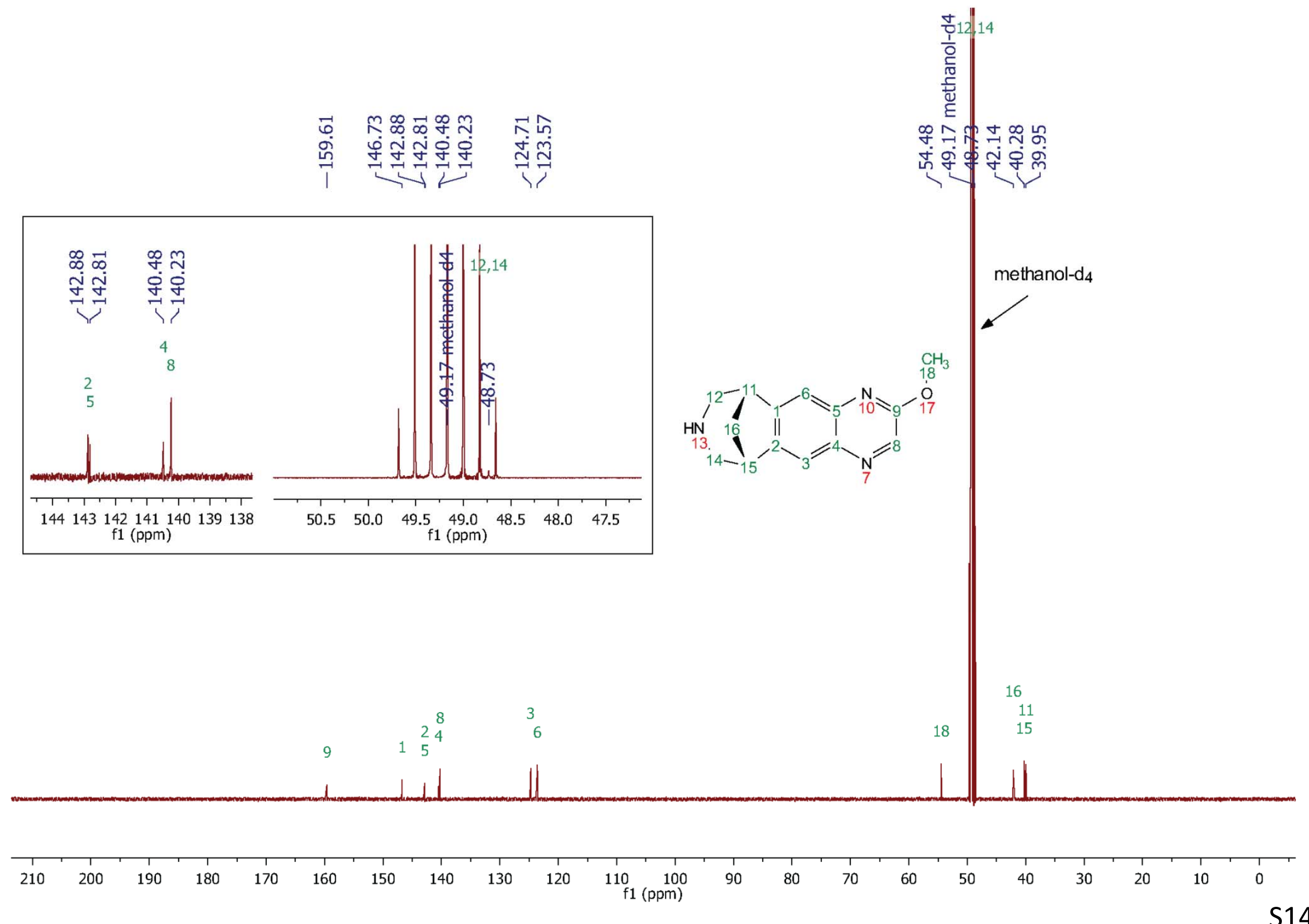
${ }^{1} \mathrm{H} /{ }^{1} \mathrm{H}$ COSY NMR spectrum of $20 \mathrm{a}\left(500 / 500 \mathrm{MHz}, \mathrm{CD}_{3} \mathrm{OD}\right)$

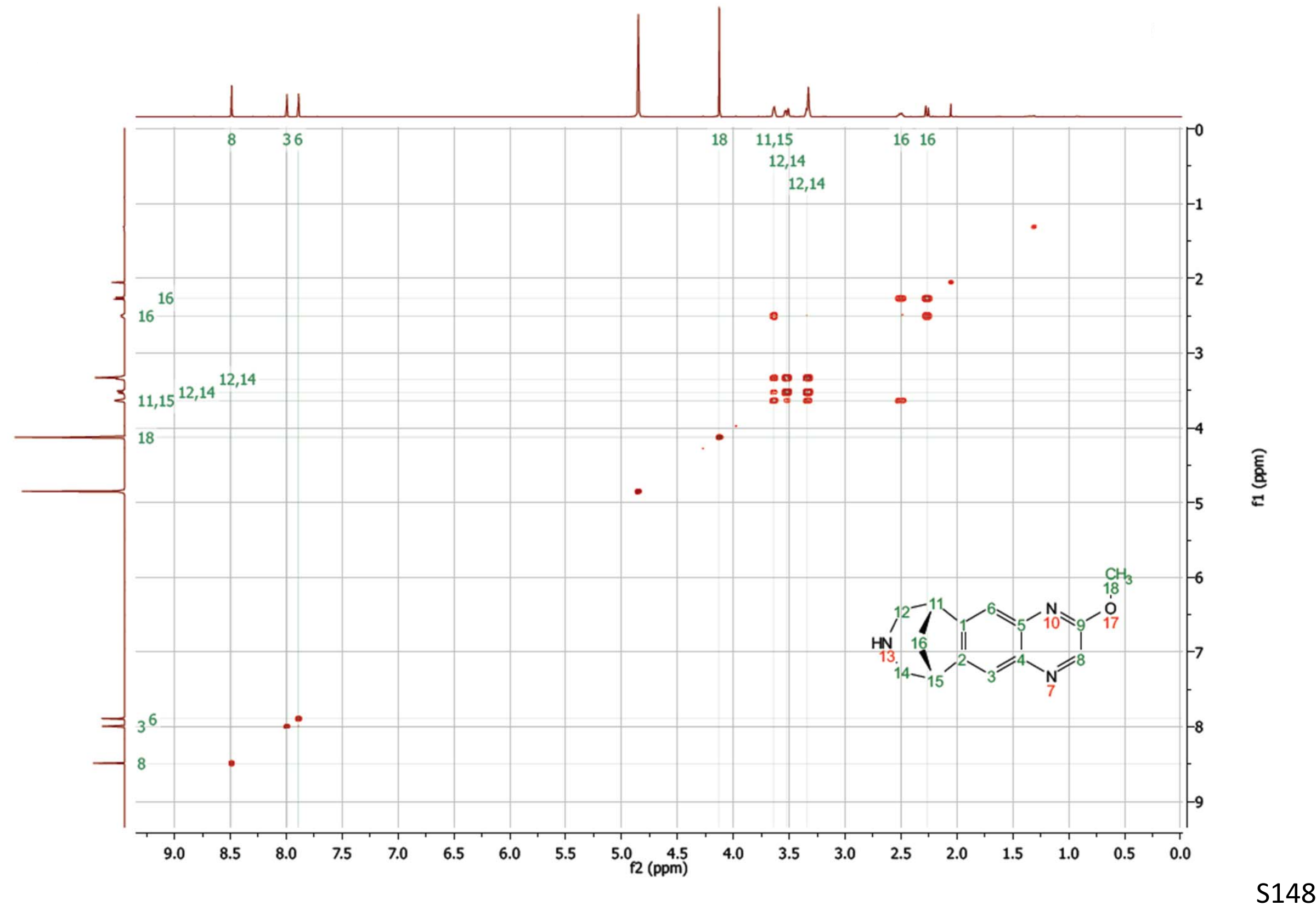


${ }^{1} \mathrm{H} /{ }^{13} \mathrm{C}$ HSQC NMR spectrum of $20 \mathrm{a}\left(500 / 125 \mathrm{MHz}, \mathrm{CD}_{3} \mathrm{OD}\right)$

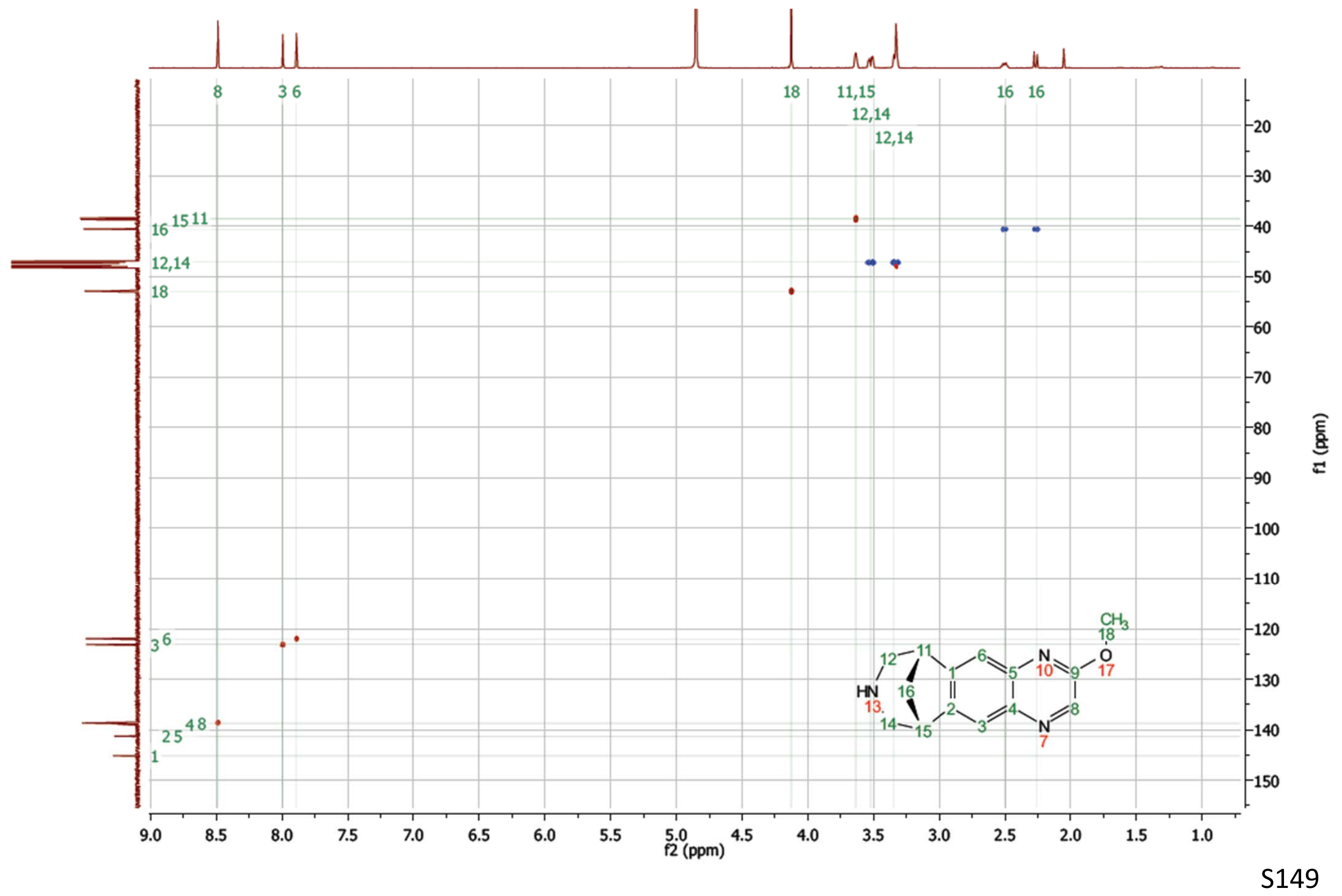


${ }^{1} \mathrm{H} /{ }^{13} \mathrm{C}$ HMBC NMR spectrum of $20 \mathrm{a}\left(500 / 125 \mathrm{MHz}, \mathrm{CD}_{3} \mathrm{OD}\right)$

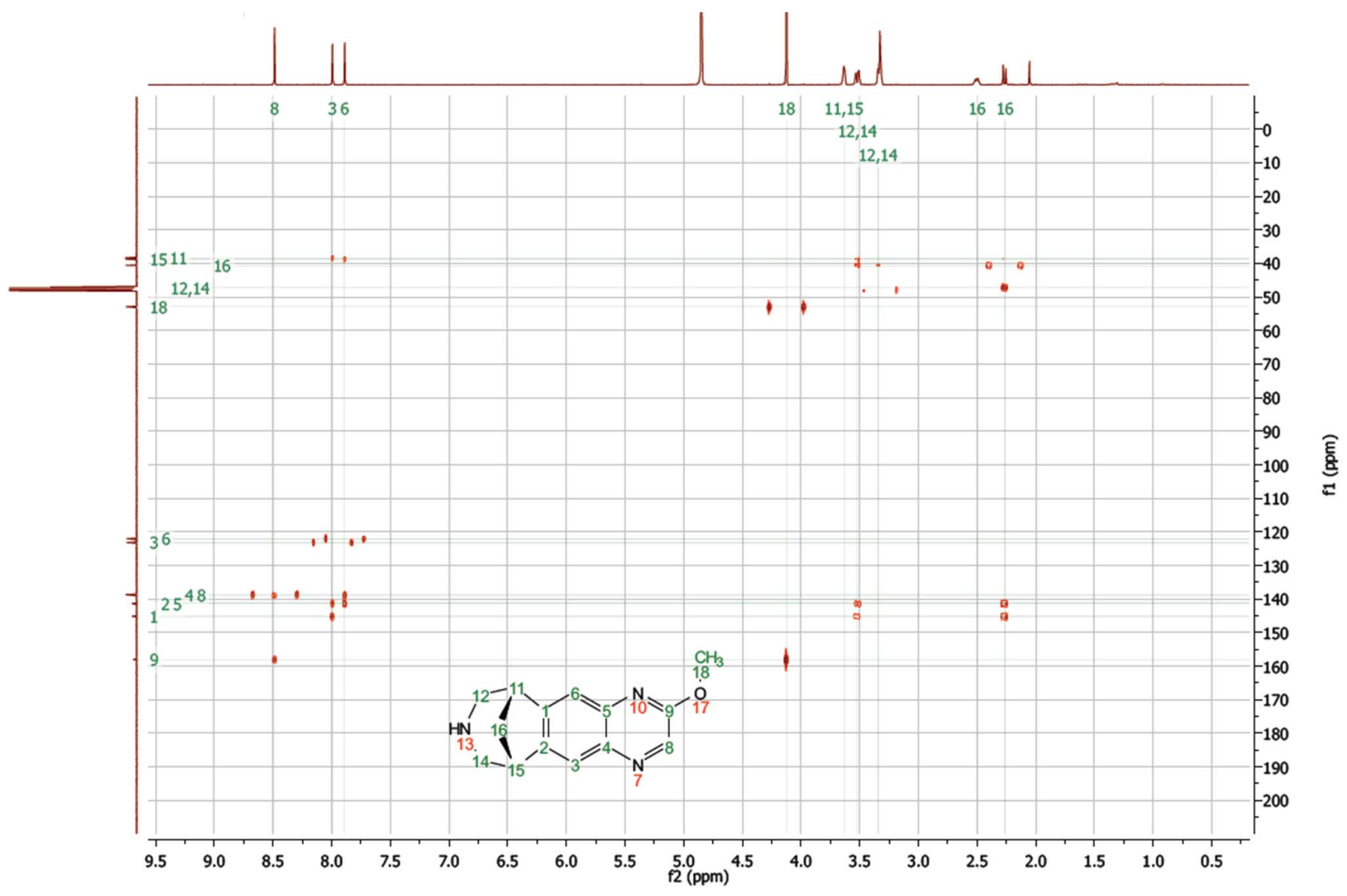


${ }^{1} \mathrm{H}$ NMR spectrum of $20 \mathrm{~b}\left(600 \mathrm{MHz}, \mathrm{CDCl}_{3}\right)$

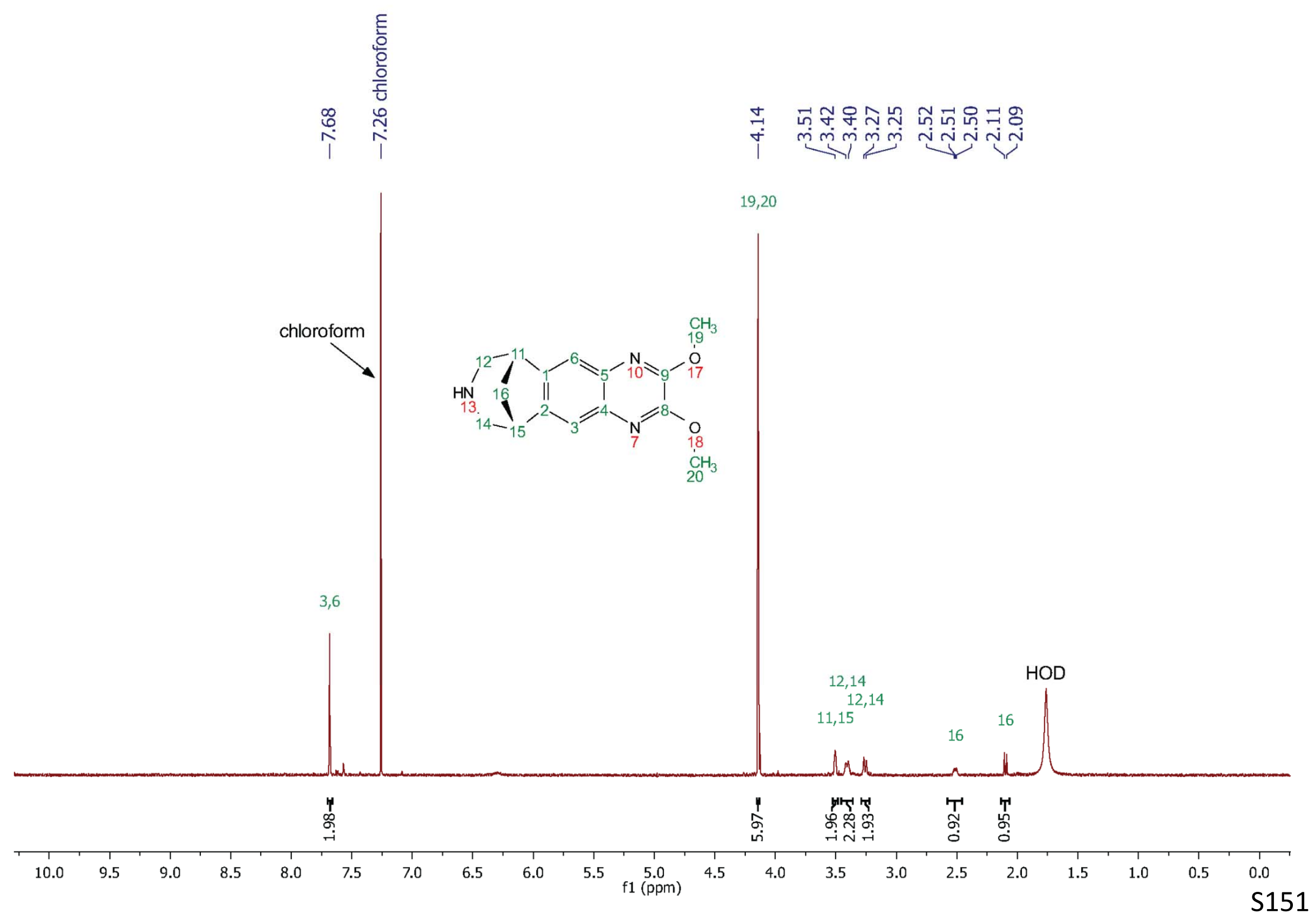


${ }^{13} \mathrm{C}$ NMR spectrum of $20 \mathrm{~b}\left(125 \mathrm{MHz}, \mathrm{CD}_{3} \mathrm{OD}\right)$

索兴兽

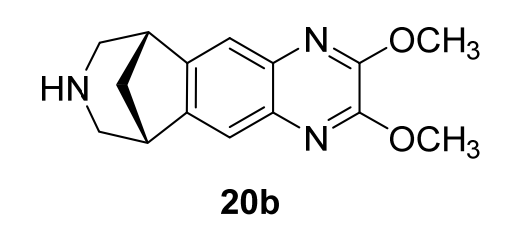

$$
\text { i }
$$$$
\text { methanol }
$$$$
\text { 䒢 }
$$
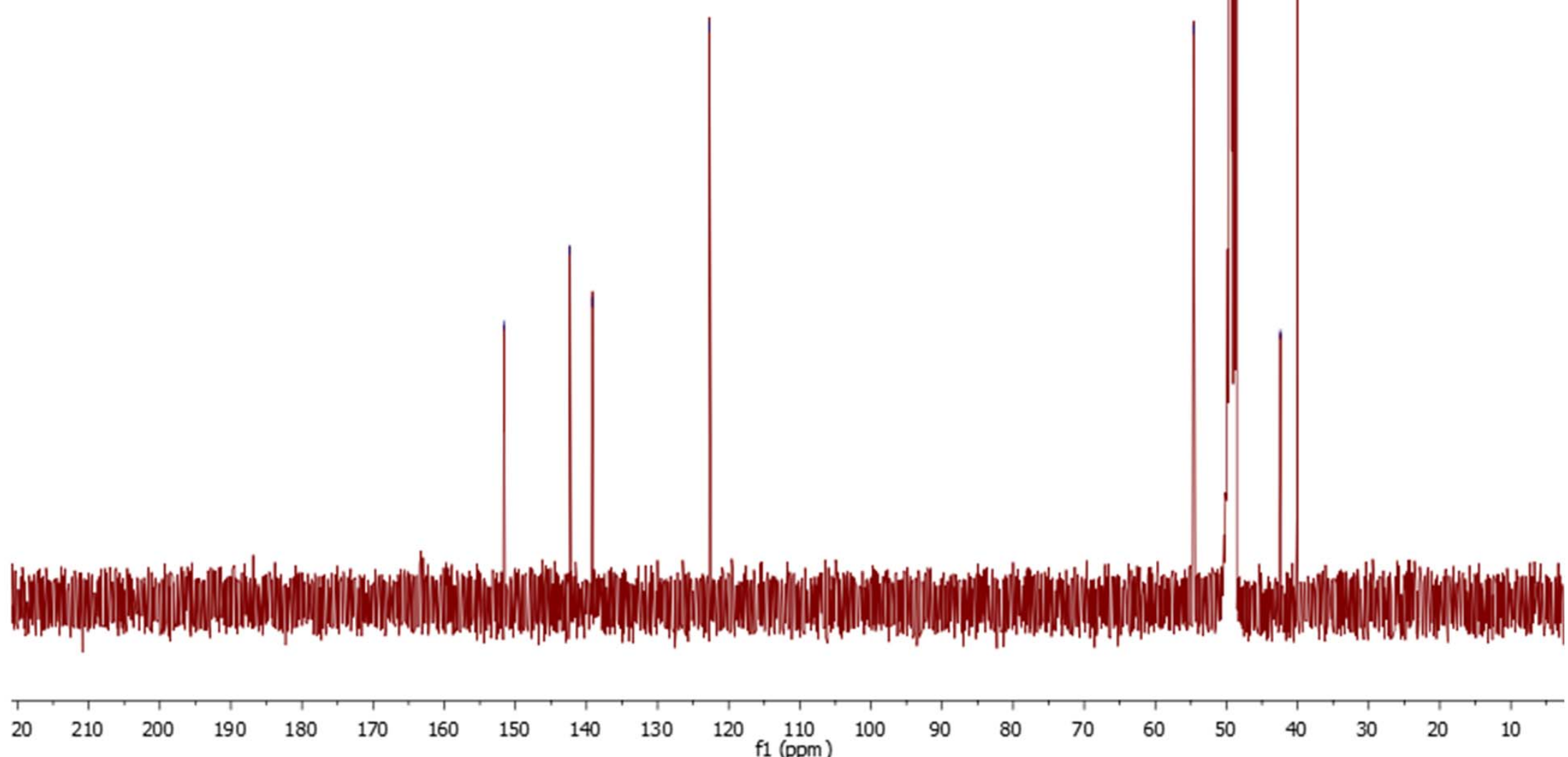
${ }^{1} \mathrm{H} /{ }^{1} \mathrm{H}$ COSY NMR spectrum of $20 \mathrm{~b}\left(600 / 600 \mathrm{MHz}, \mathrm{CDCl}_{3}\right)$

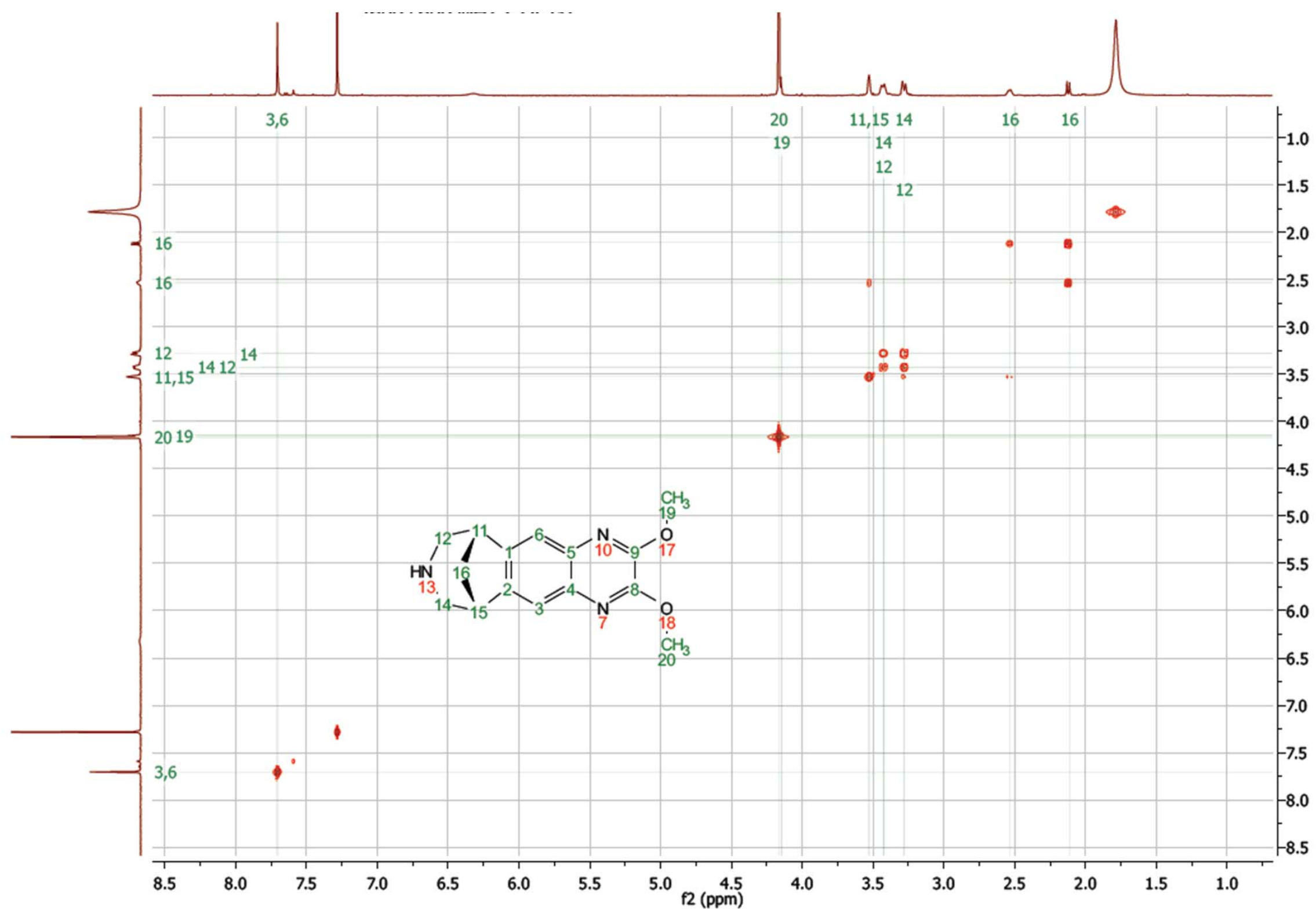


${ }^{1} \mathrm{H} /{ }^{13} \mathrm{C}$ HSQC NMR spectrum of 20b $\left(600 / 150 \mathrm{MHz}, \mathrm{CDCl}_{3}\right)$

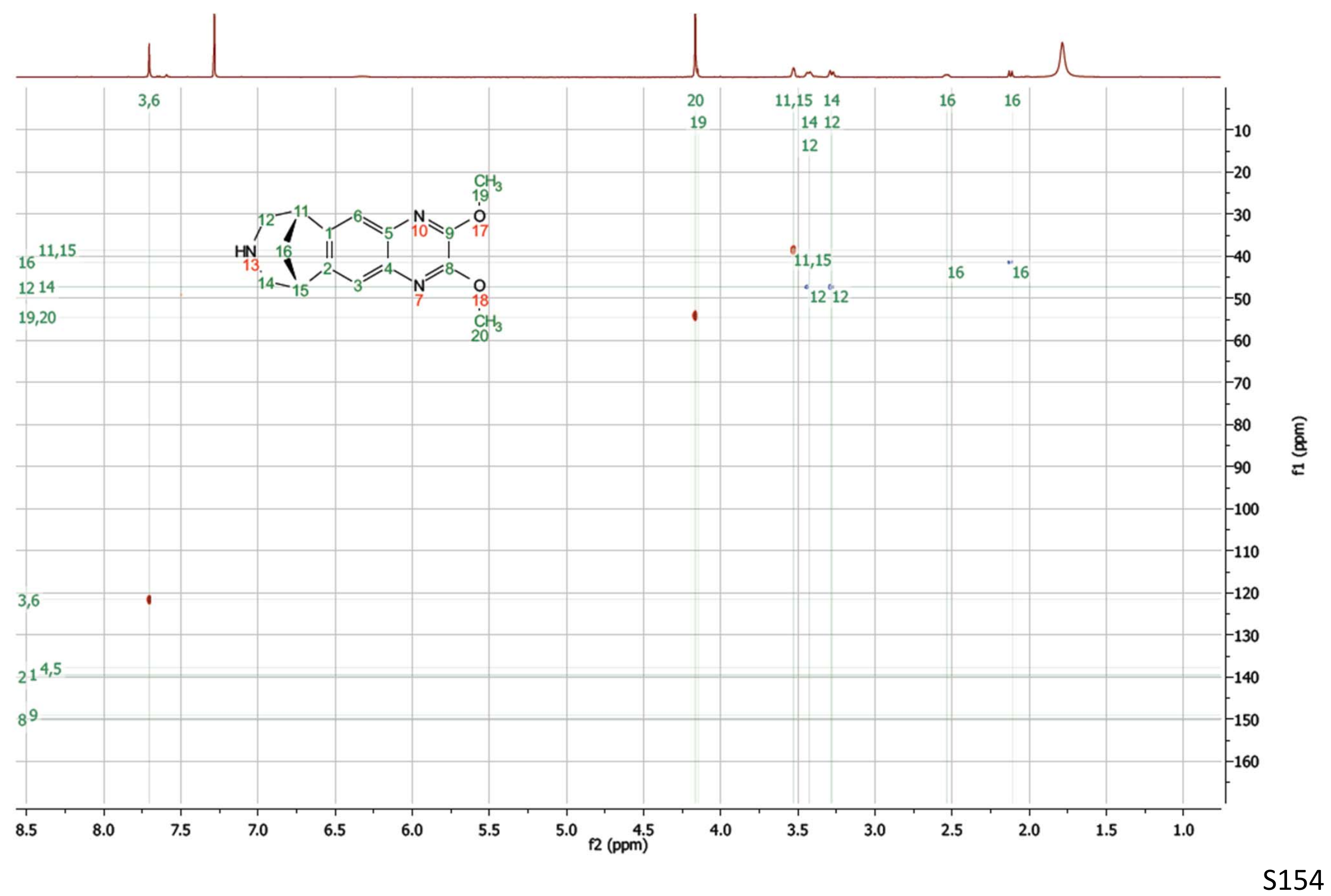


${ }^{1} \mathrm{H} /{ }^{13} \mathrm{C} \mathrm{HMBC}$ NMR spectrum of $20 \mathrm{~b}\left(600 / 150 \mathrm{MHz}, \mathrm{CDCl}_{3}\right)$

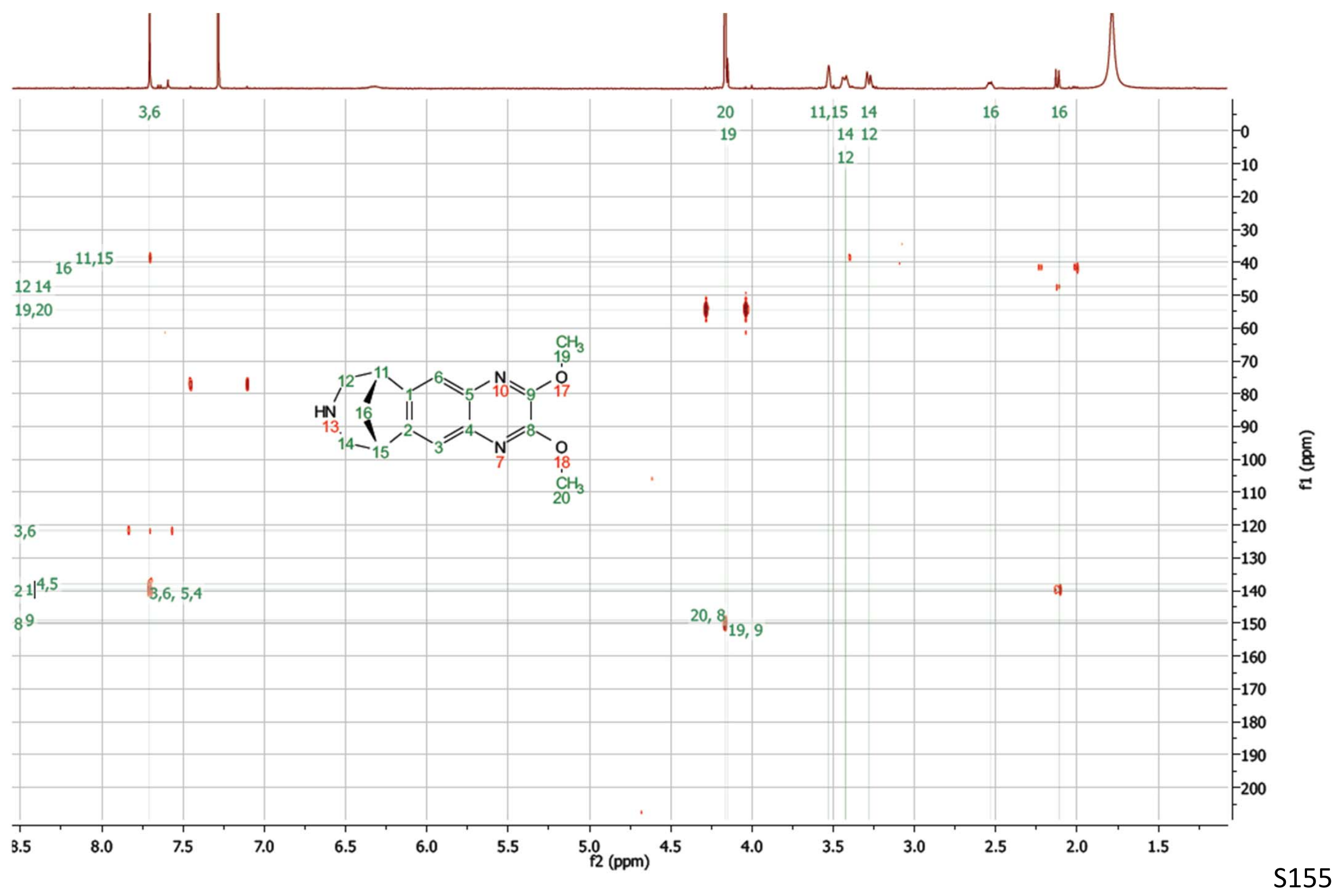




\section{${ }^{1} \mathrm{H}$ NMR spectrum of $21 \mathrm{a}\left(600 \mathrm{MHz}, \mathrm{CDCl}_{3}\right)$}

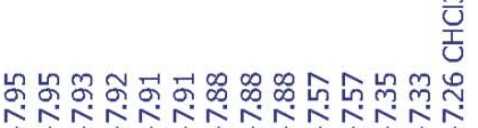

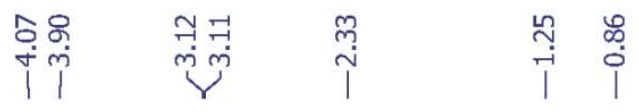
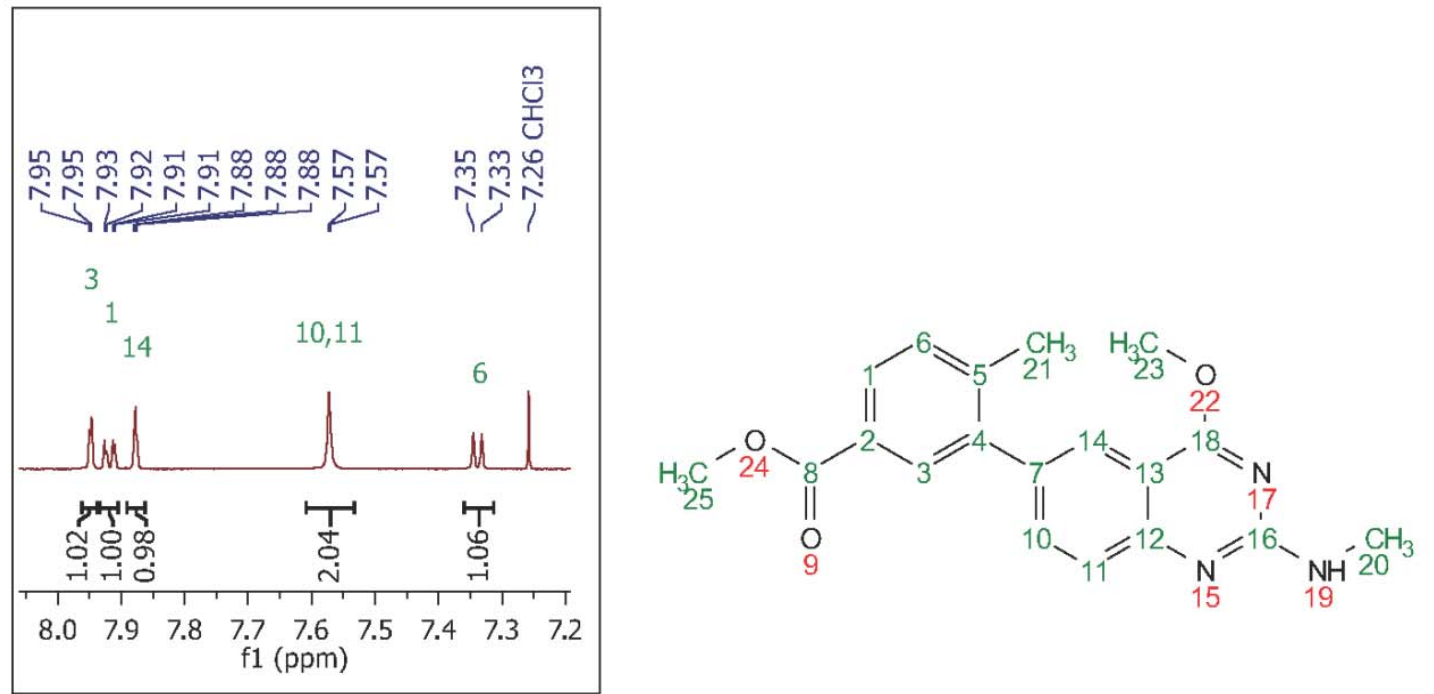

25

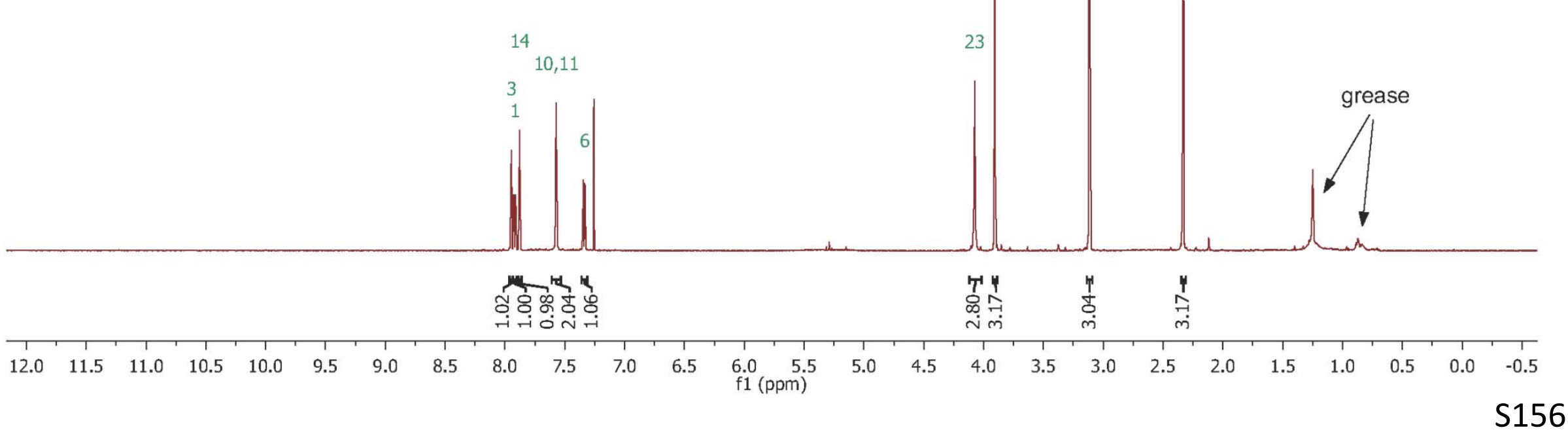


${ }^{13} \mathrm{C}$ NMR spectrum of $21 \mathrm{a}\left(150 \mathrm{MHz}, \mathrm{CDCl}_{3}\right)$
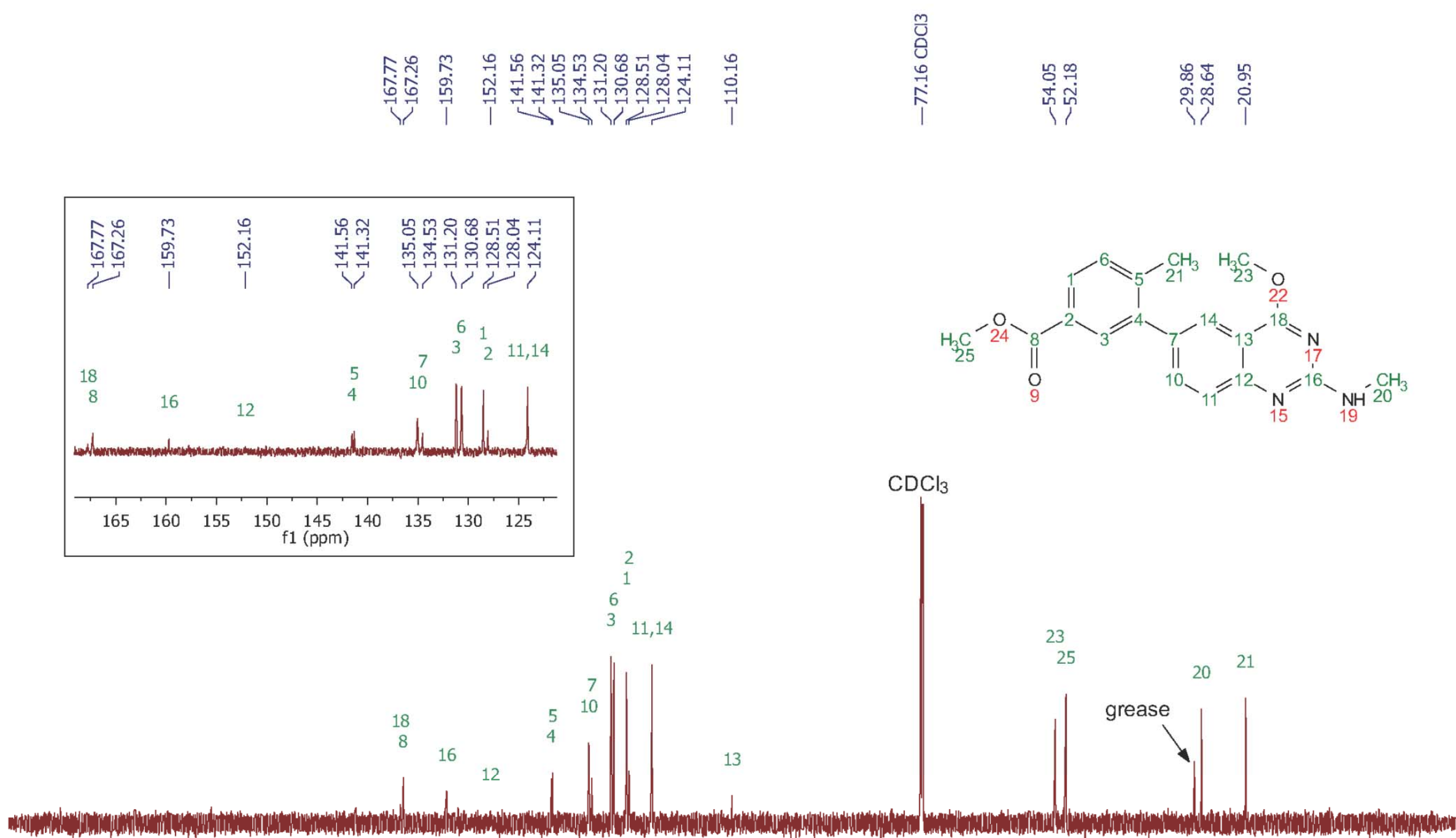

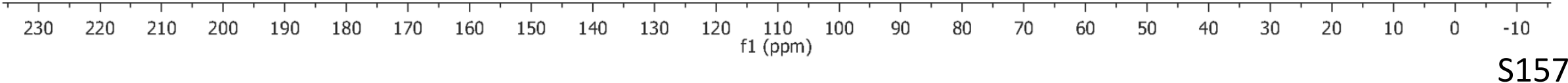


${ }^{1} \mathrm{H} /{ }^{1} \mathrm{H}$ COSY NMR spectrum of $21 \mathrm{a}\left(600 / 600 \mathrm{MHz}, \mathrm{CDCl}_{3}\right)$

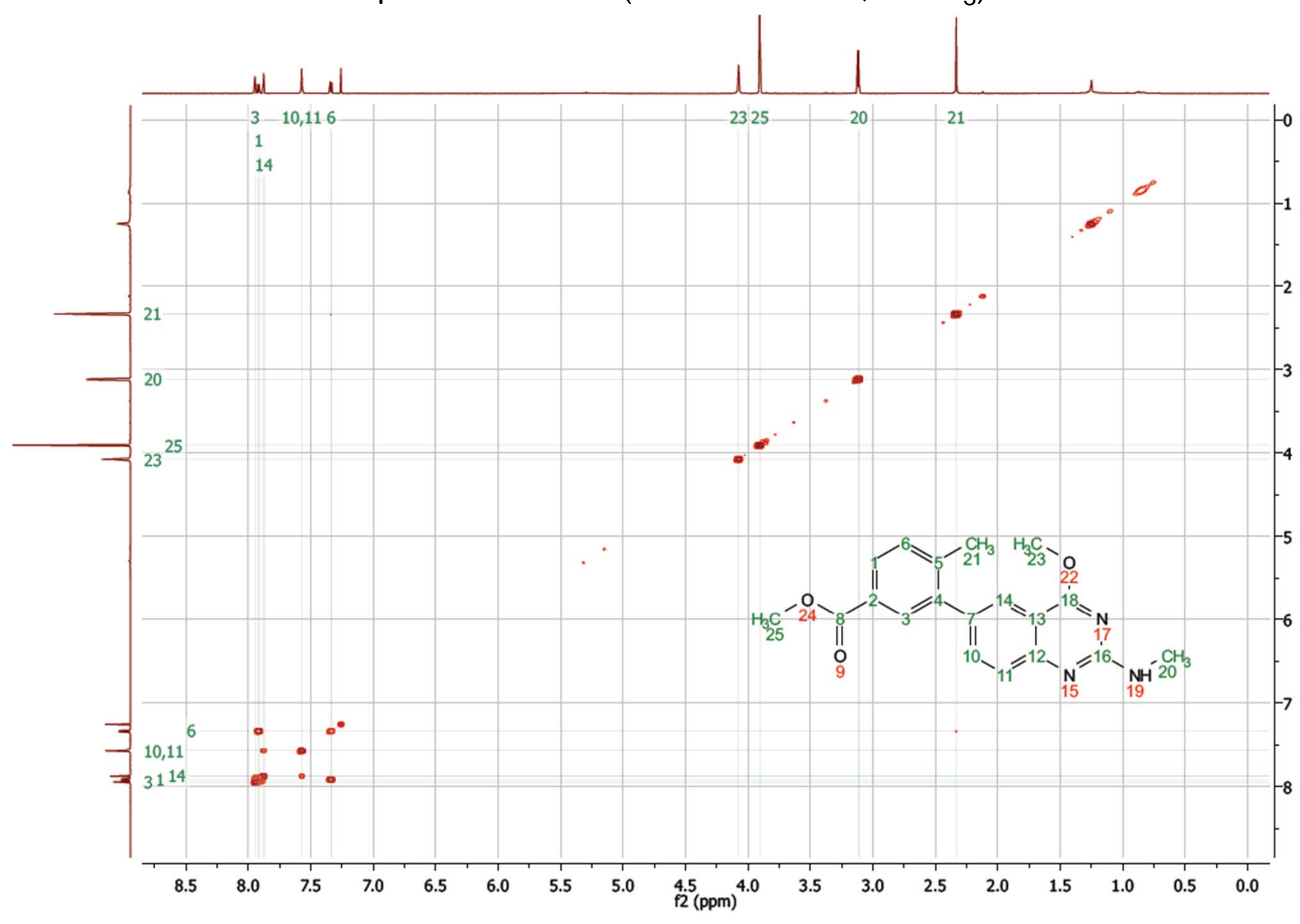


${ }^{1} \mathrm{H} /{ }^{13} \mathrm{C}$ HSQC NMR spectrum of 21a $\left(600 / 150 \mathrm{MHz}, \mathrm{CDCl}_{3}\right)$

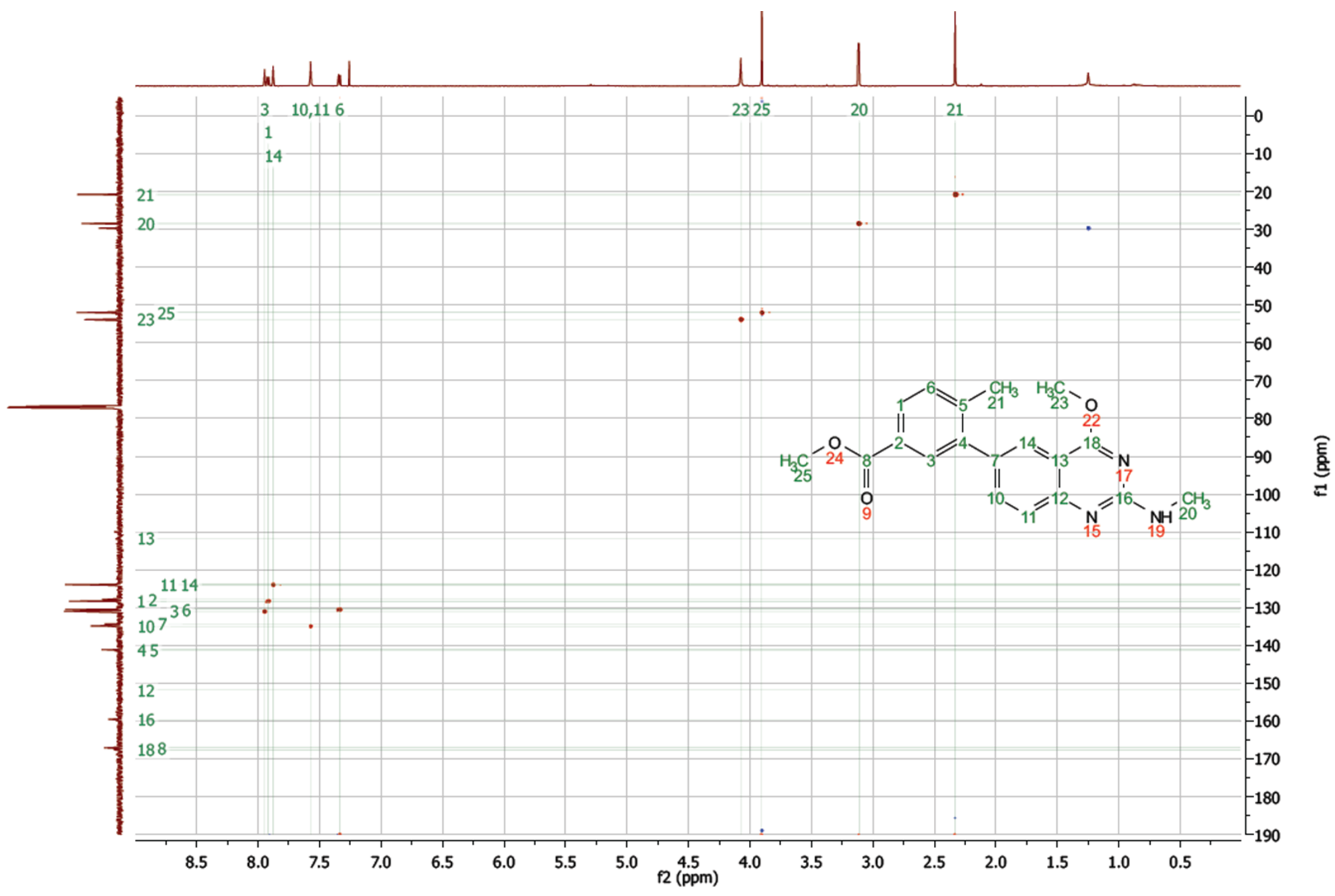


${ }^{1} \mathrm{H} /{ }^{13} \mathrm{C} \mathrm{HMBC}$ NMR spectrum of 21a $\left(600 / 150 \mathrm{MHz}, \mathrm{CDCl}_{3}\right)$

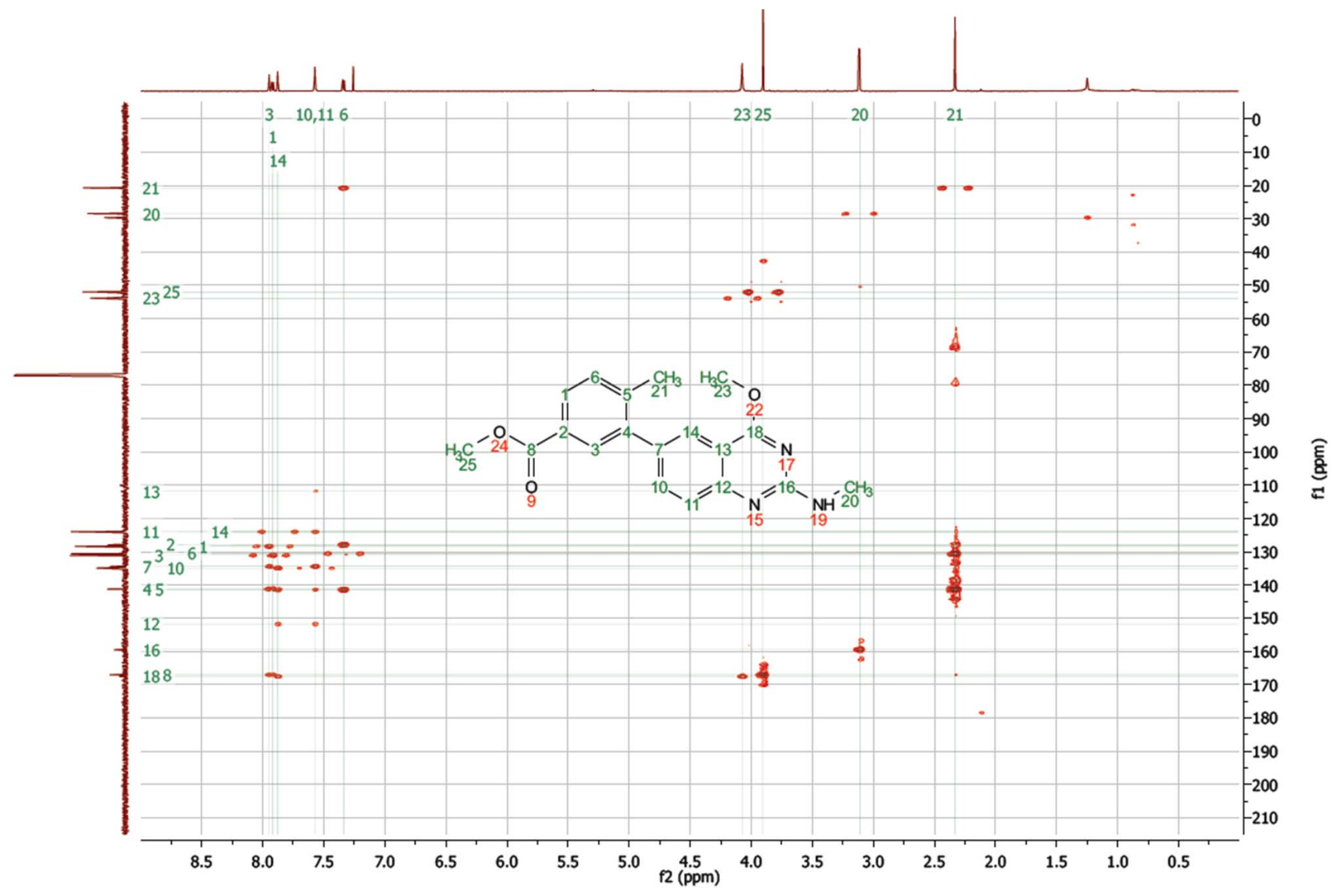


${ }^{1} \mathrm{H} /{ }^{1} \mathrm{H}$ ROESY NMR spectrum of $21 \mathrm{a}\left(600 / 600 \mathrm{MHz}, \mathrm{CDCl}_{3}\right)$

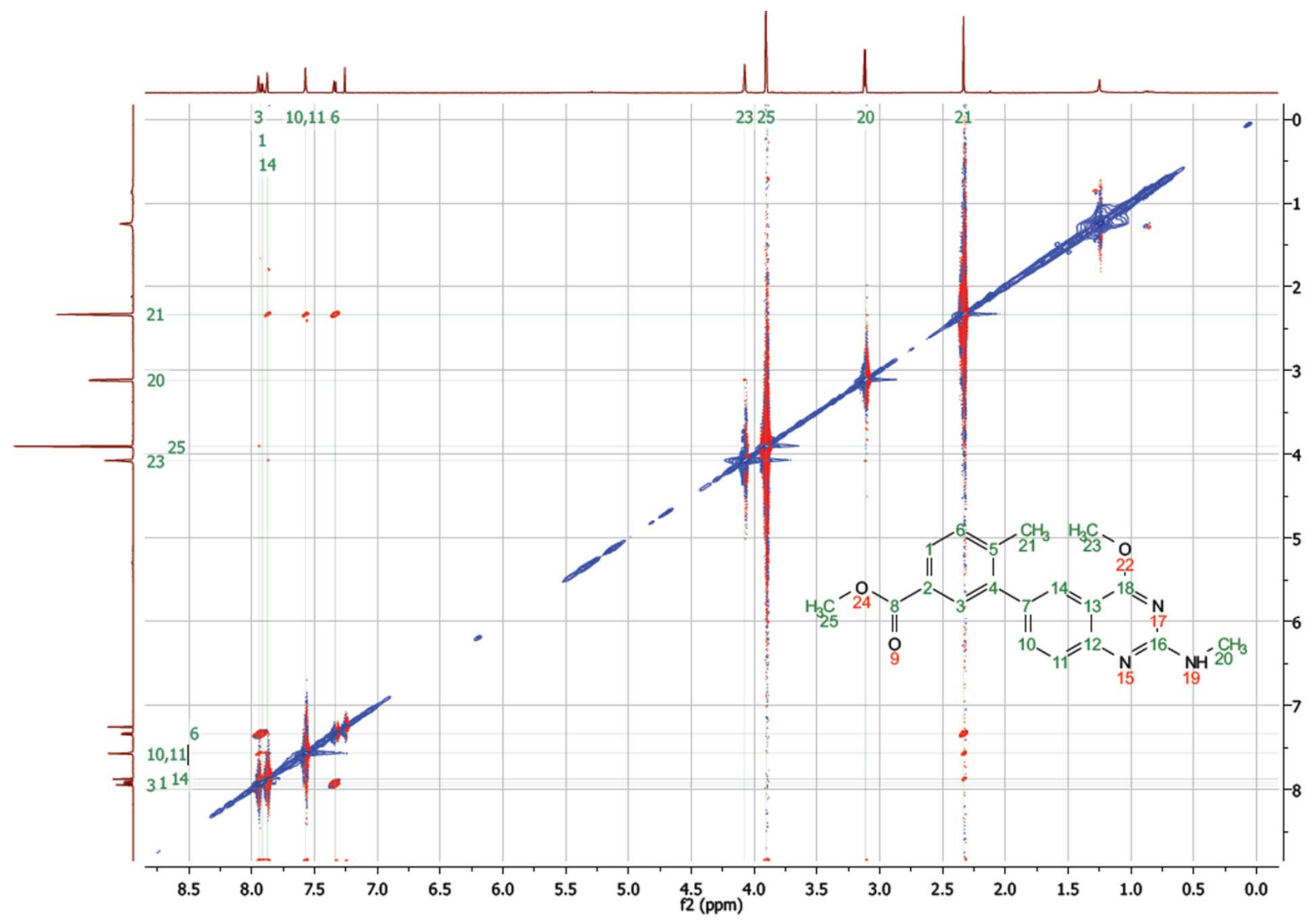


${ }^{1} \mathrm{H}$ NMR spectrum of $22 \mathrm{a} / \mathrm{b} \bullet T F A\left(600 \mathrm{MHz}\right.$, DMSO- $\left.\mathrm{d}_{6}\right)$

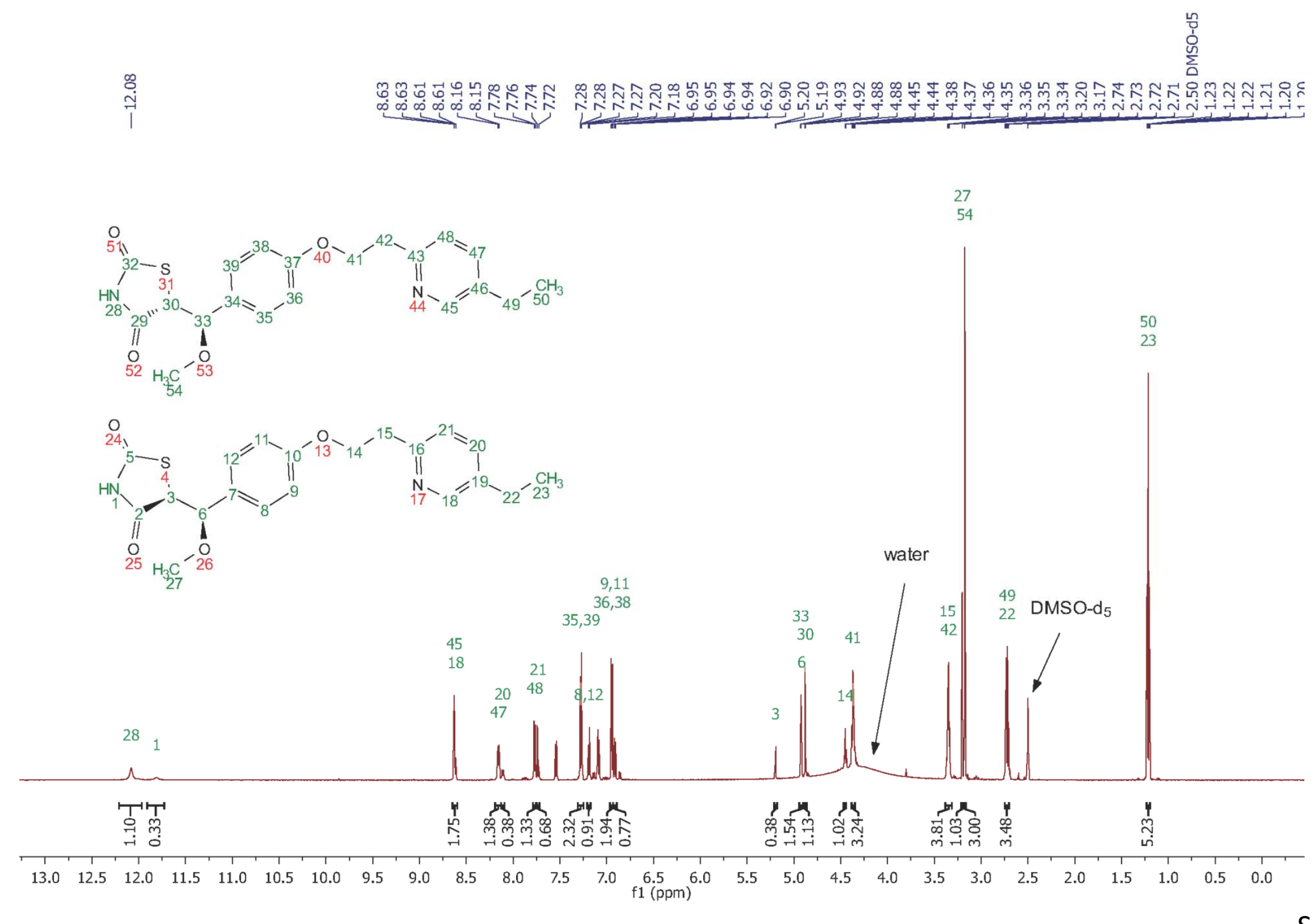


${ }^{13} \mathrm{C}$ NMR spectrum of 22a/b•TFA (150 MHz, DMSO- $\mathrm{d}_{6}$ )

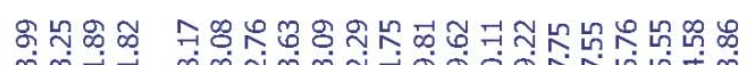

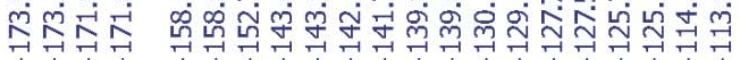
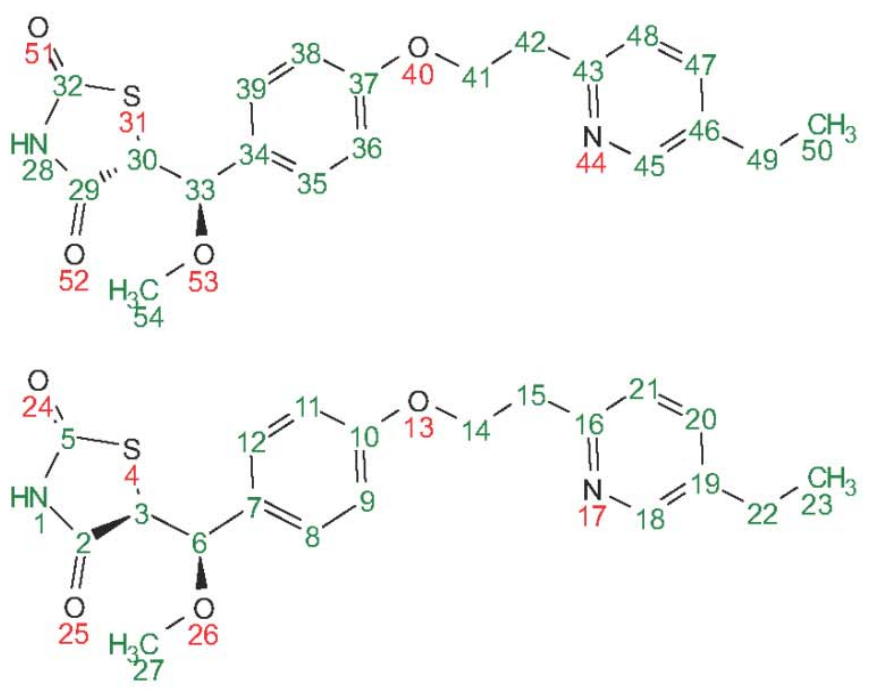

DMSO-d 6

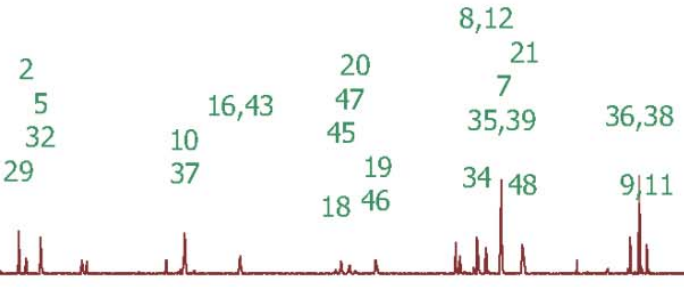


${ }^{1} \mathrm{H} /{ }^{1} \mathrm{H}$ COSY NMR spectrum of 22a/b•TFA $\left(600 / 600 \mathrm{MHz}\right.$, DMSO- $\left.\mathrm{d}_{6}\right)$

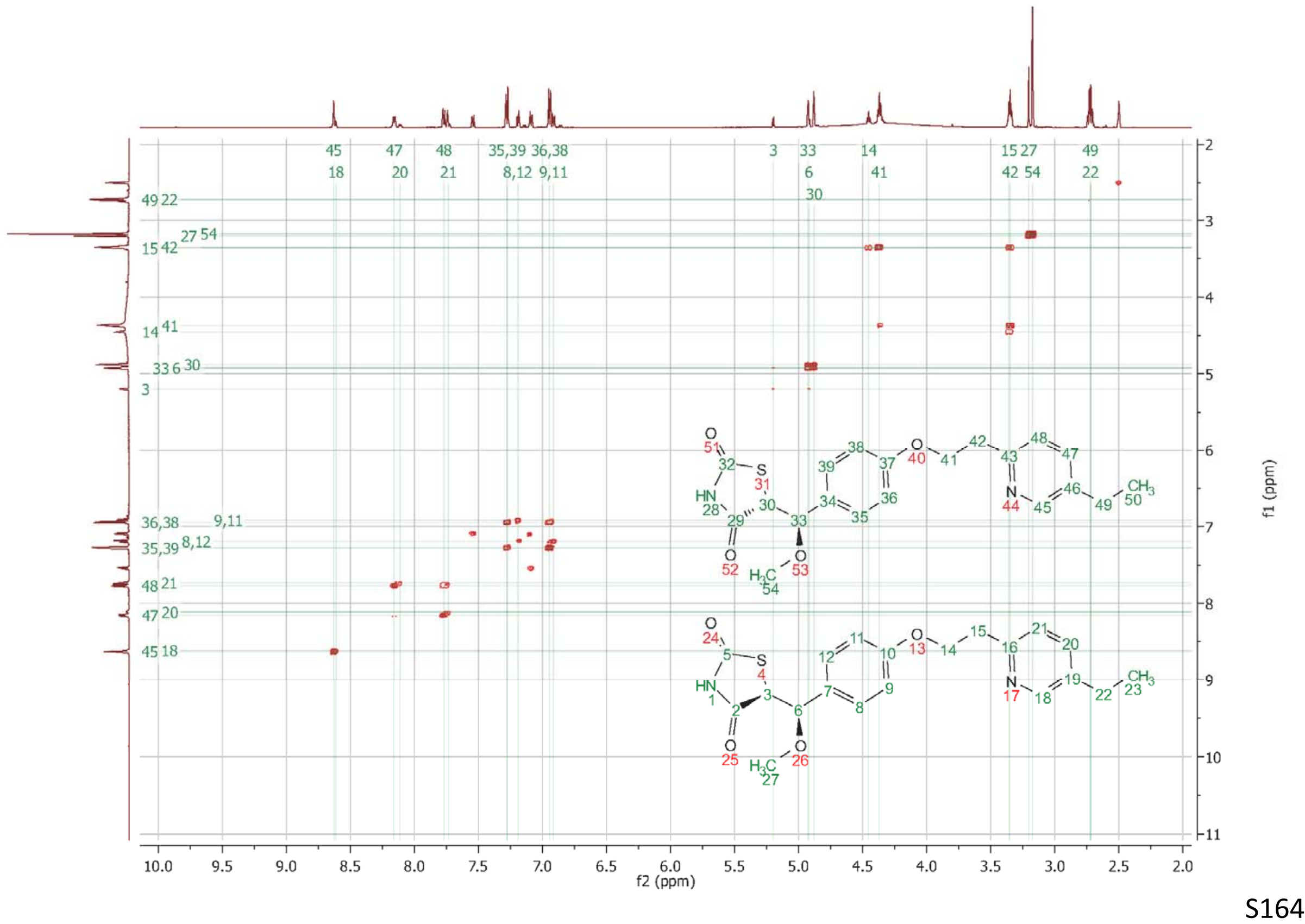


${ }^{1} \mathrm{H} /{ }^{13} \mathrm{C}$ HSQC NMR spectrum of 22a/b•TFA (600 / $150 \mathrm{MHz}$, DMSO-d $\left.\mathrm{d}_{6}\right)$

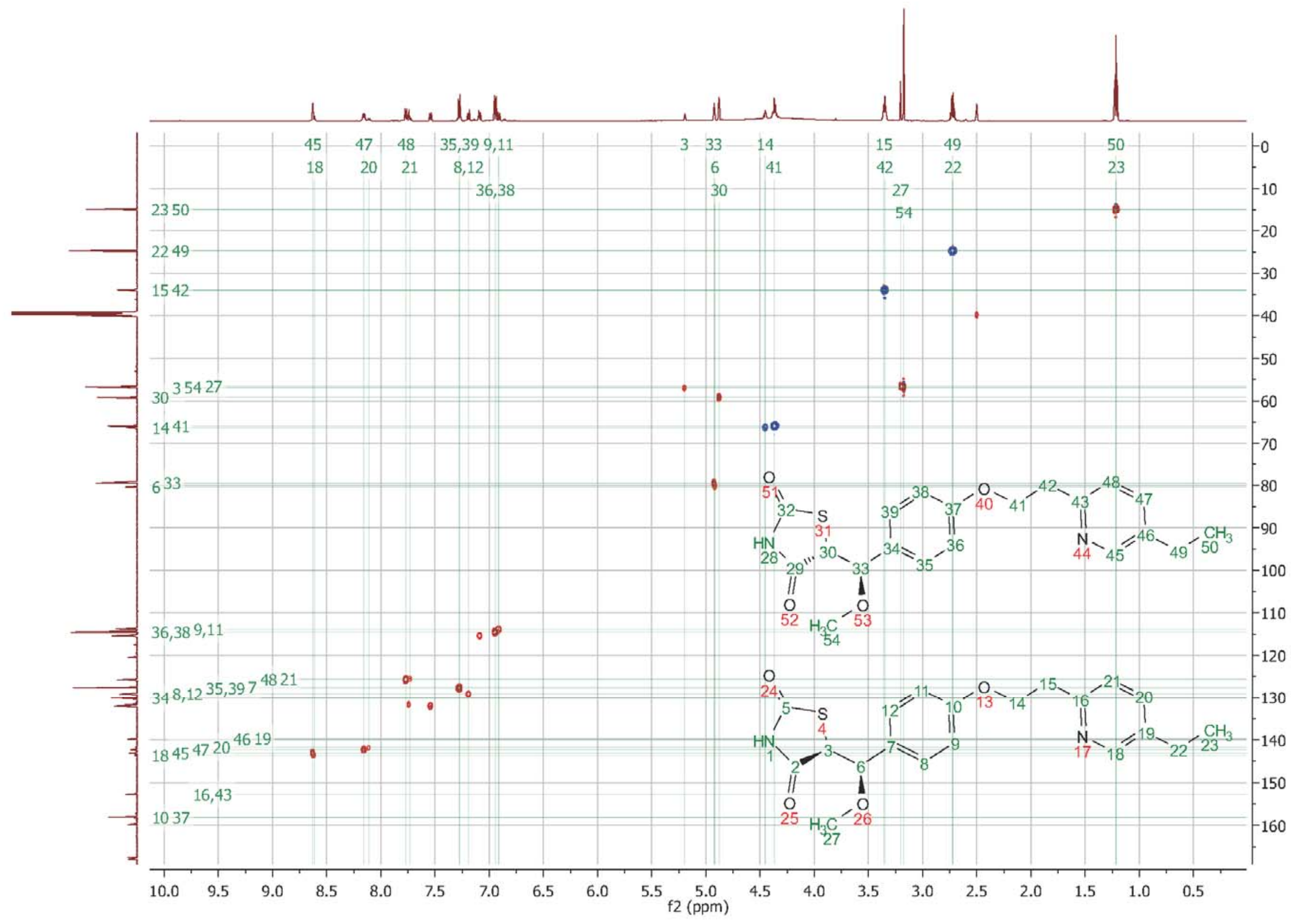


${ }^{1} \mathrm{H} /{ }^{13} \mathrm{C}$ HMBC NMR spectrum of 22a/b•TFA (600 / $150 \mathrm{MHz}$, DMSO-d $\left.\mathrm{d}_{6}\right)$

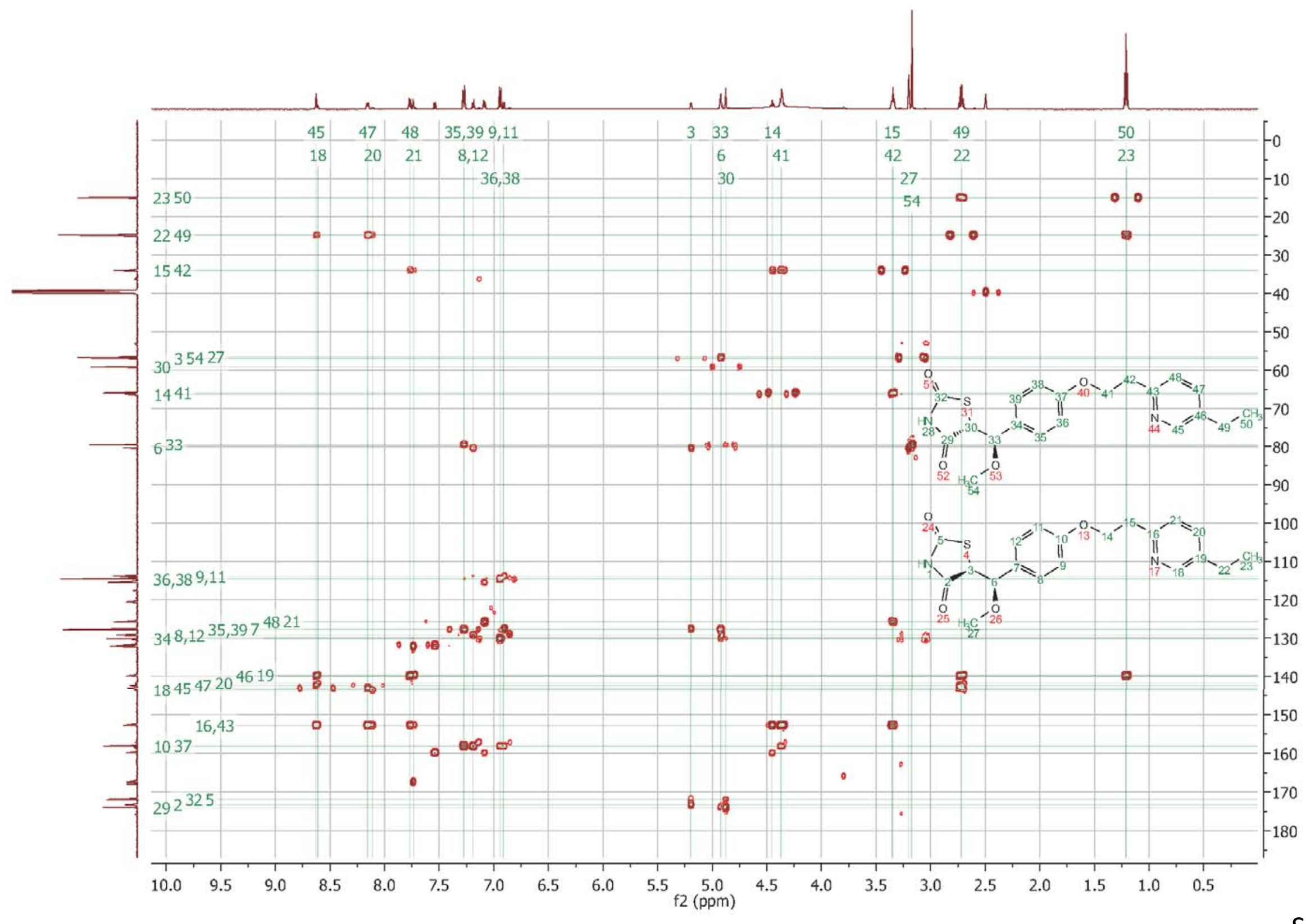


${ }^{1} \mathrm{H} /{ }^{1} \mathrm{H}$ ROESY NMR spectrum of 22a/b•TFA (600 / $600 \mathrm{MHz}$, DMSO-d $\left.\mathrm{d}_{6}\right)$

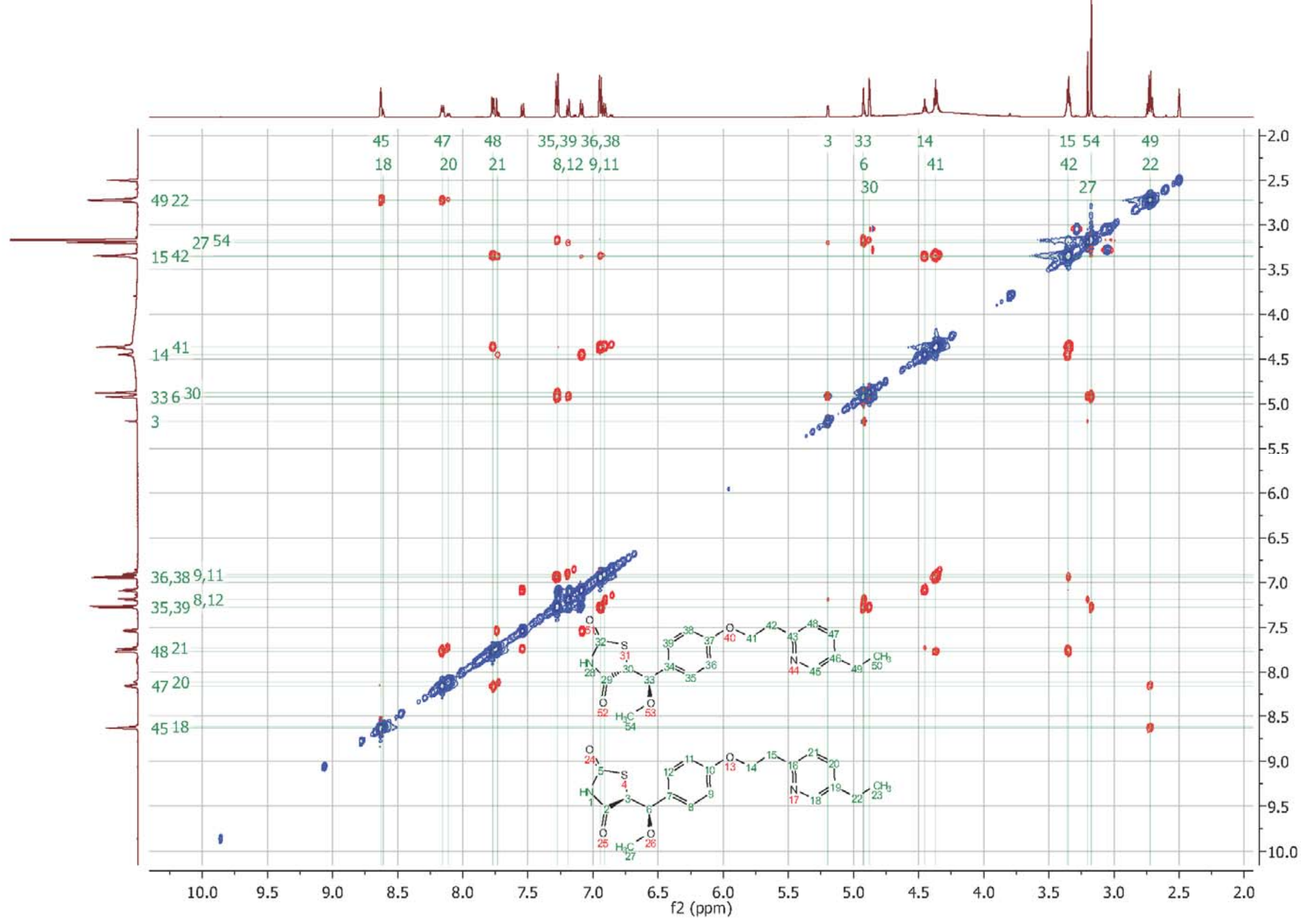


${ }^{1} \mathrm{H}$ NMR spectrum of 22c•TFA (600 MHz, DMSO- $\mathrm{d}_{6}$ )

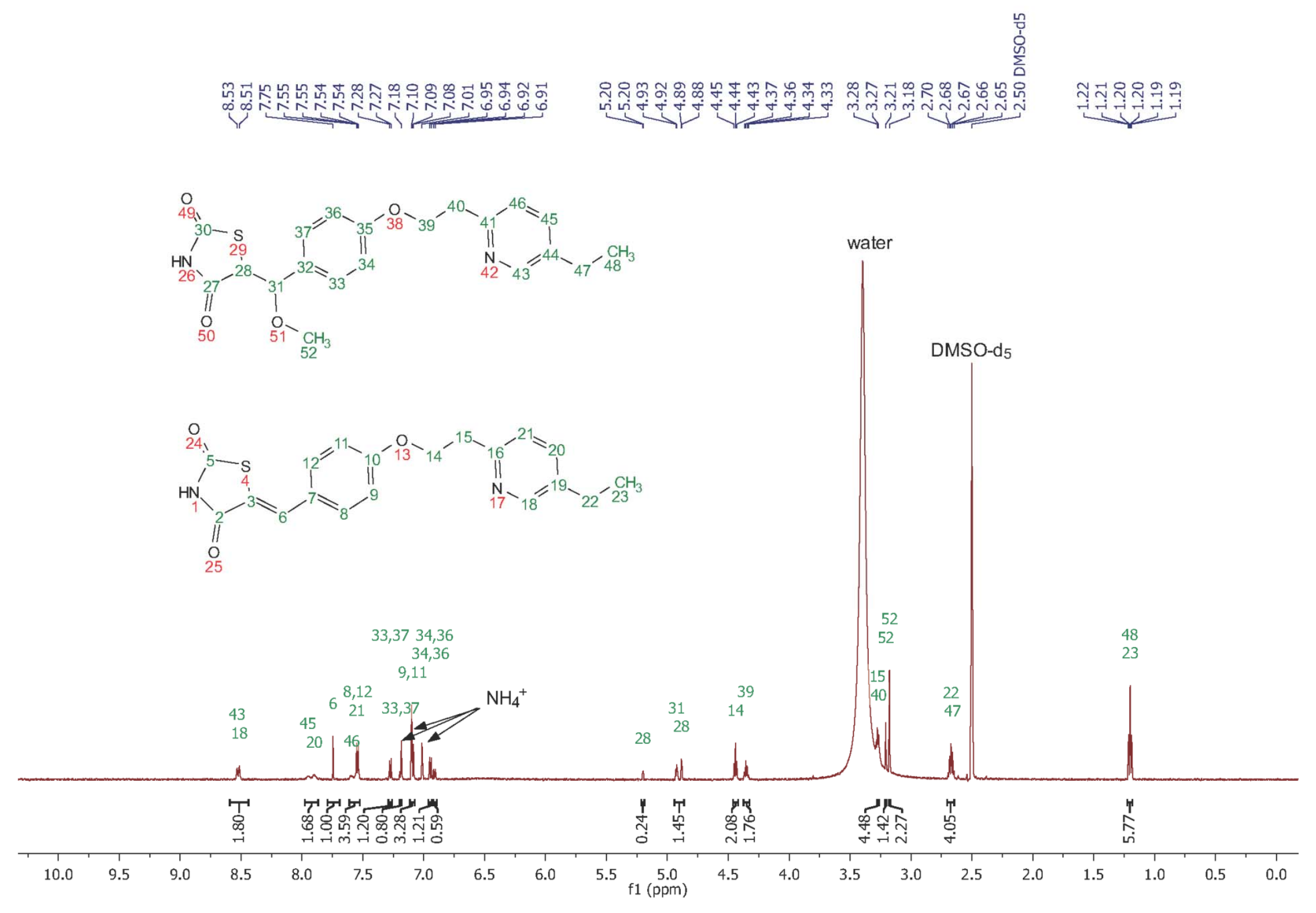


${ }^{13} \mathrm{C}$ NMR spectrum of 22c•TFA (150 MHz, DMSO-d 6 )
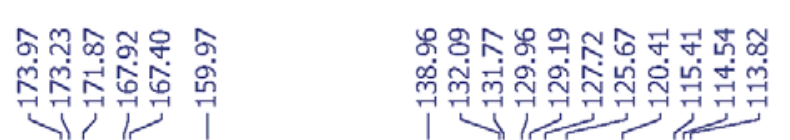

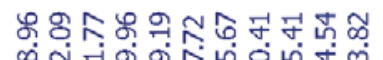

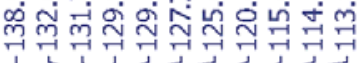
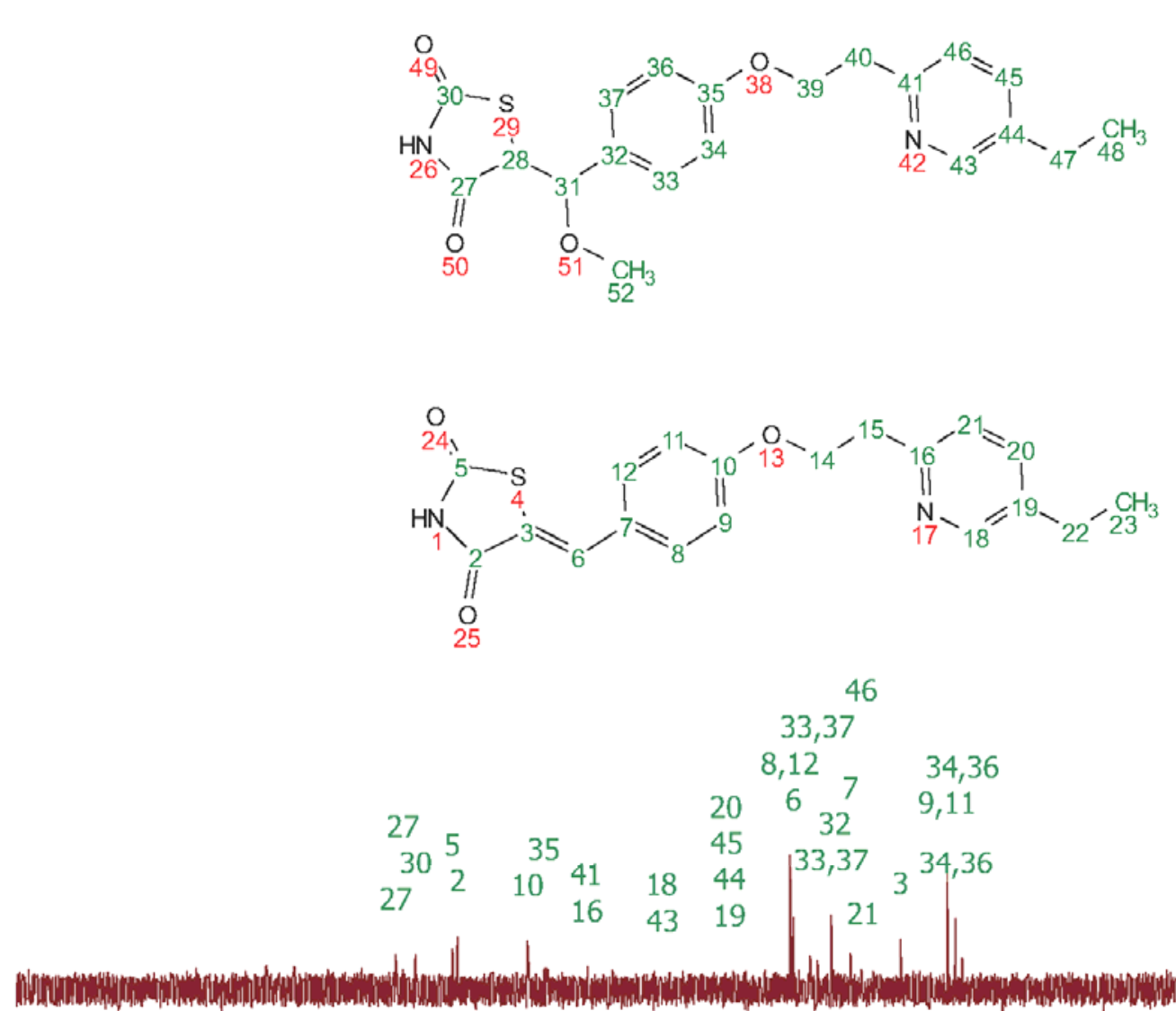
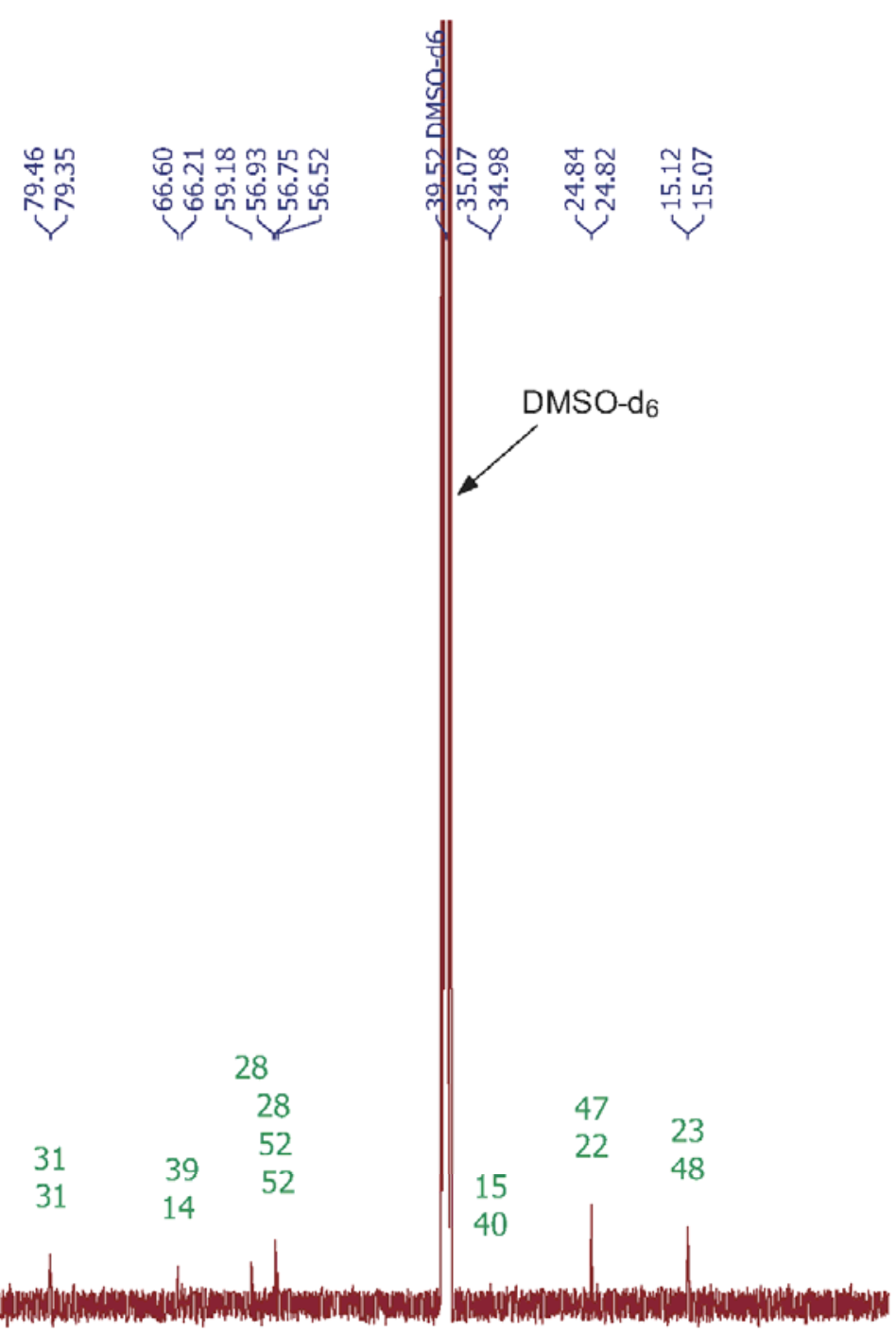
${ }^{1} \mathrm{H} /{ }^{1} \mathrm{H}$ COSY NMR spectrum of 22c•TFA (600 / $600 \mathrm{MHz}$, DMSO-d ${ }_{6}$ )

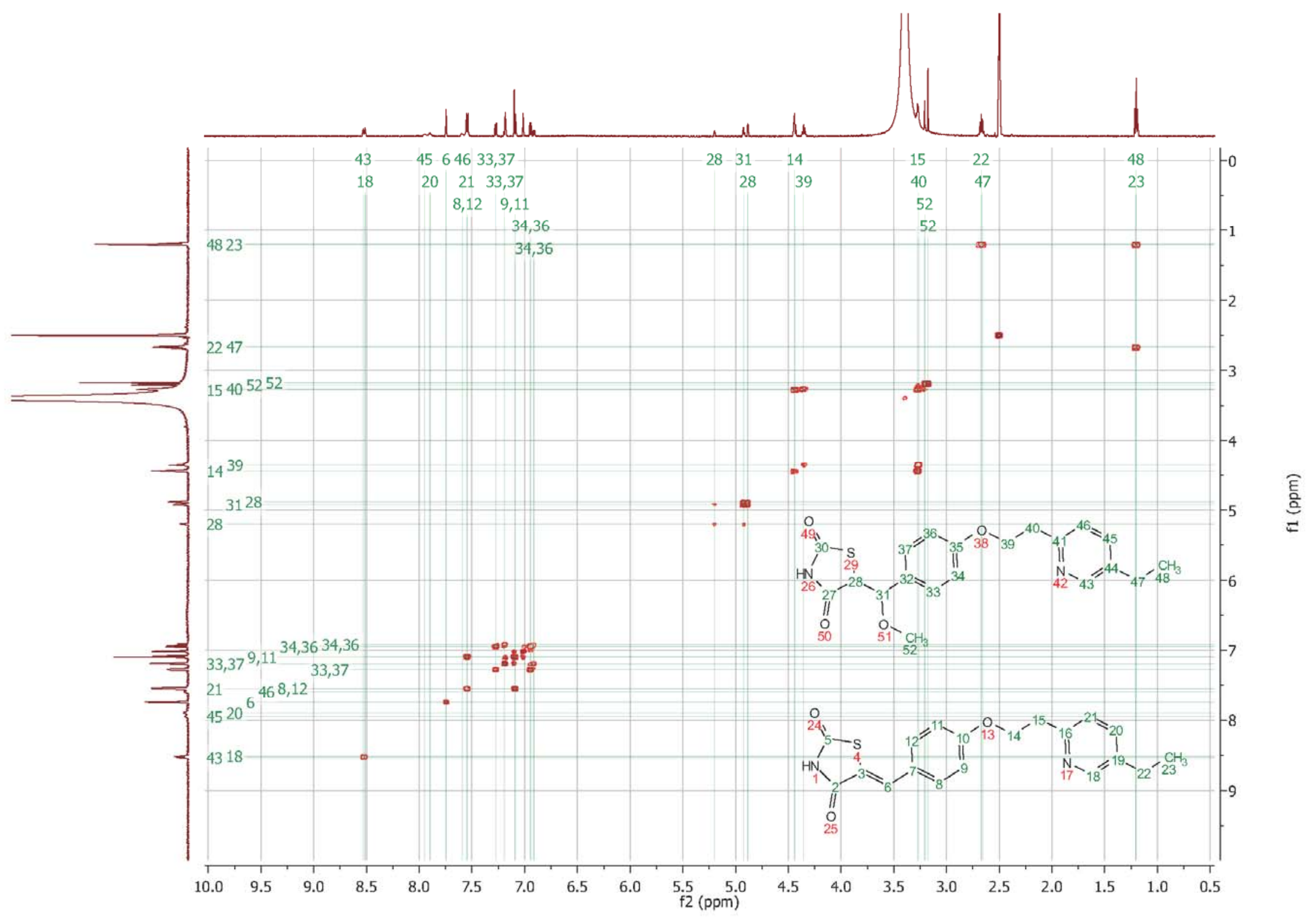


${ }^{1} \mathrm{H} /{ }^{13} \mathrm{C}$ HSQC NMR spectrum of 22c•TFA (600 / $150 \mathrm{MHz}, \mathrm{DMSO}-\mathrm{d}_{6}$ )

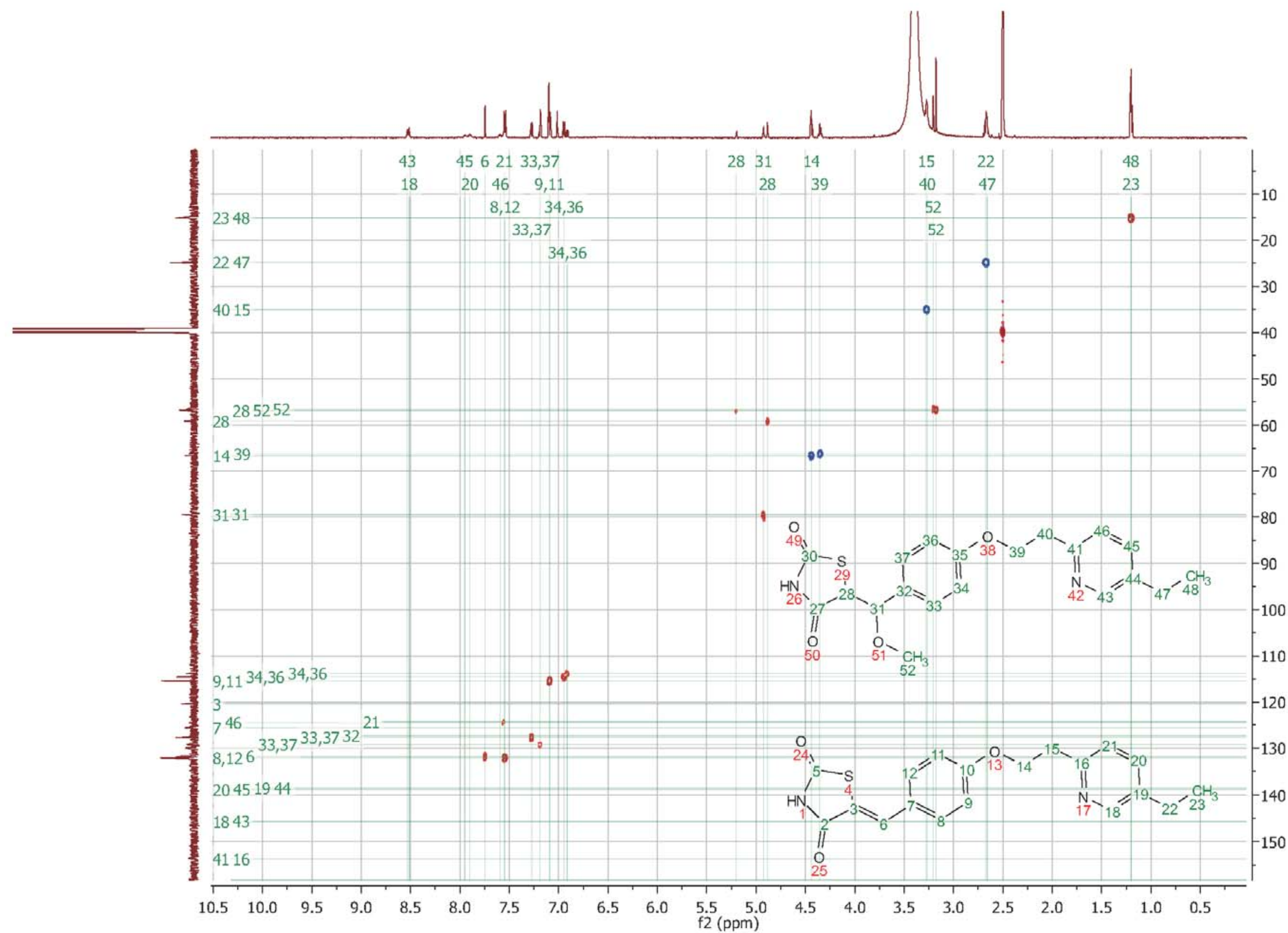


${ }^{1} \mathrm{H} /{ }^{13} \mathrm{C}$ HMBC NMR spectrum of 22c•TFA (600 / $150 \mathrm{MHz}$, DMSO- $\left.\mathrm{d}_{6}\right)$

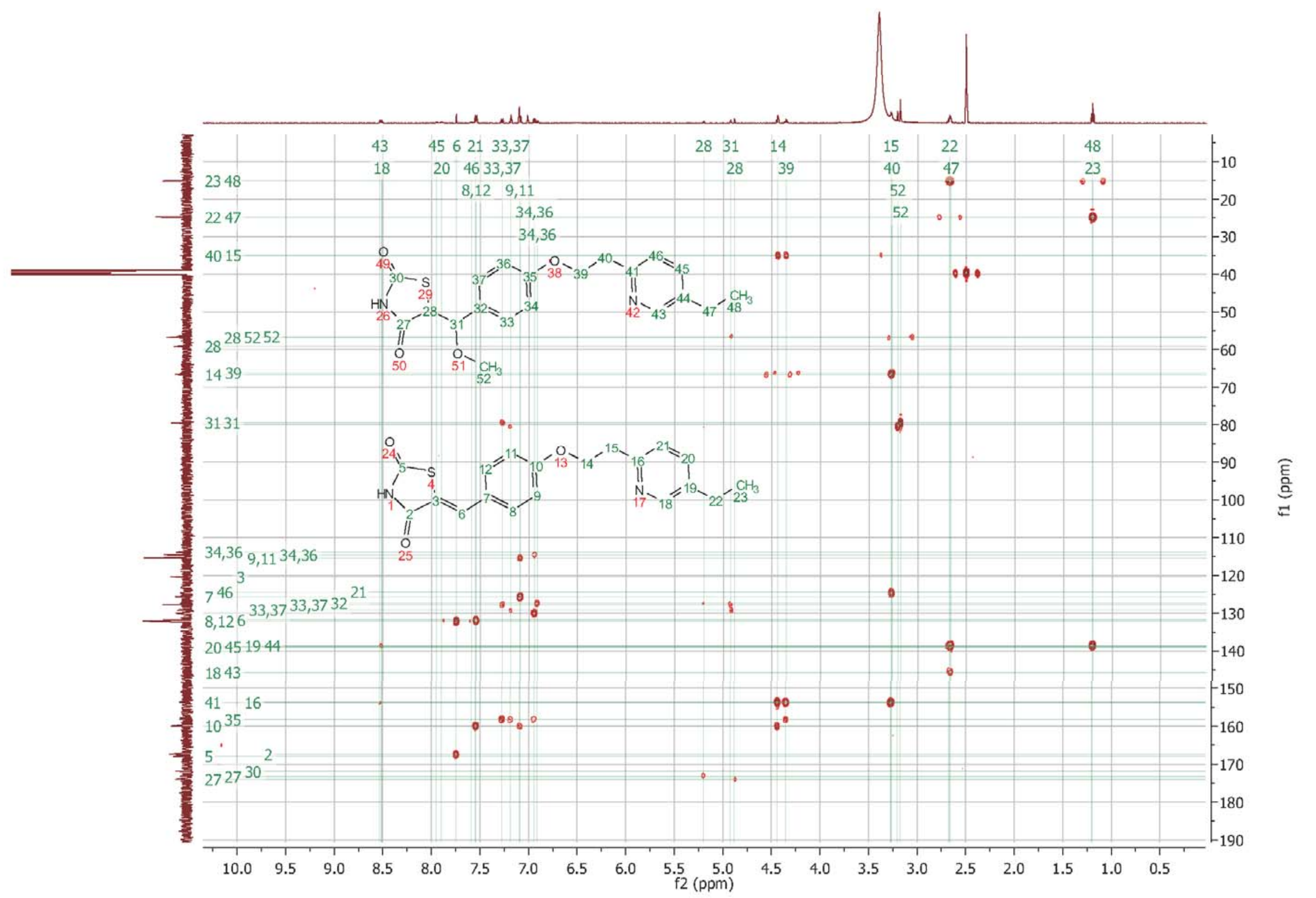


${ }^{1} \mathrm{H} /{ }^{1} \mathrm{H}$ ROESY NMR spectrum of 22c•TFA $\left(600 / 600 \mathrm{MHz}\right.$, DMSO-d $\left.\mathrm{d}_{6}\right)$

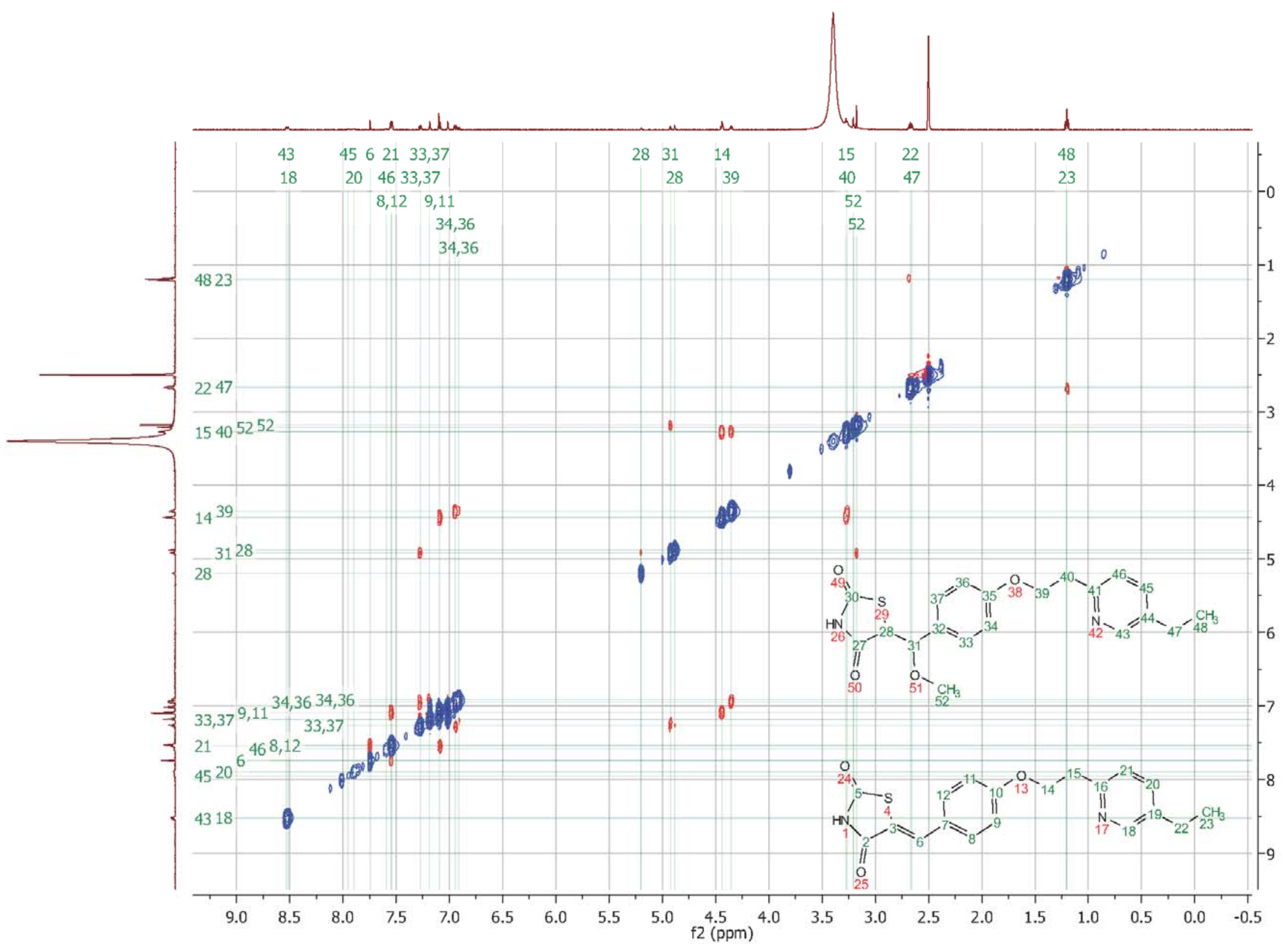

